\title{
An Approach Based On Wavelet Decomposition And Neural Network For ECG Noise Reduction
}

\author{
A Thesis \\ presented to \\ the Faculty of California Polytechnic State University, \\ San Luis Obispo
}

\author{
In Partial Fulfillment \\ of the Requirement for the Degree \\ Master of Science in Electrical Engineering
}

By

Suranai Poungponsri

June 2009 
(C) 2009

Suranai Poungponsri

ALL RIGHTS RESERVED 


\section{CommitTeE MeMbership}

TITLE:

AUTHOR:

Date Submitted:

Committee Chair:

Committee Member:

Committee Member:
An Approach Based On Wavelet Decomposition and Neural Network for ECG Noise Reduction Suranai Poungponsri

June 2009

Xiao-Hua (Helen) Yu, Ph.D.

Fred W. DePiero, Ph.D.

John Seng, Ph.D. 


\section{ABSTRACT}

\section{An Approach Based On Wavelet Decomposition and Neural Network for ECG Noise Reduction}

\section{Suranai Poungponsri}

Electrocardiogram (ECG) signal processing has been the subject of intense research in the past years, due to its strategic place in the detection of several cardiac pathologies. However, ECG signal is frequently corrupted with different types of noises such as $60 \mathrm{~Hz}$ power line interference, baseline drift, electrode movement and motion artifact, etc. In this thesis, a hybrid two-stage model based on the combination of wavelet decomposition and artificial neural network is proposed for ECG noise reduction based on excellent localization features: wavelet transform and the adaptive learning ability of neural network. Results from the simulations validate the effectiveness of this proposed method. Simulation results on actual ECG signals from MIT-BIH arrhythmia database [30] show this approach yields improvement over the un-filtered signal in terms of signal-to-noise ratio (SNR).

Keywords: Wavelet Decomposition, Artificial Neural Network, Function Approximation, Noise Reduction, ECG, Adaptive Filter. 


\section{ACKNOWLEDGMENT}

I would like to thank Dr. Xiao-Hua (Helen) Yu for her continuous guidance in the completion of this work, Dr. Fred W. DePiero and Dr. John Seng for their advice in parts of the work as well as for serving on my committee.

I am grateful to Neil Patel and Marnie Parker for their helps in proofreading this work. 


\section{TABLE OF CONTENTS}

Chapter 1: Introduction ....................................................................

Chapter 2: Artificial Neural Network ..................................................9

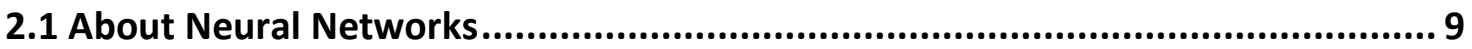

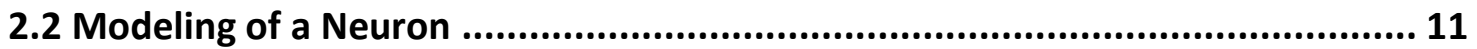

2.3 Architectures of Neural Networks................................................................... 13

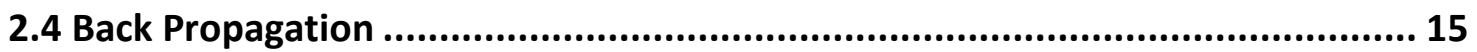

2.4.1 Weight Adjustment for Output Layer.......................................................... 16

2.4.1 Weight Adjustment for Hidden Layers .......................................................... 17

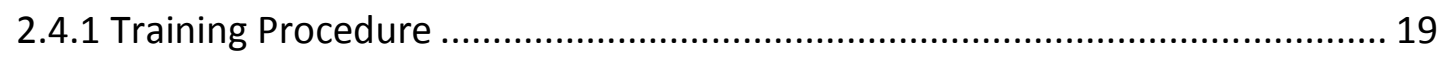

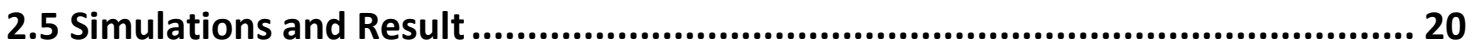

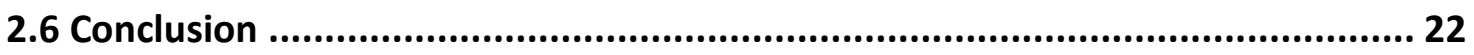

Chapter 3: Introduction to Wavelet.....................................................28

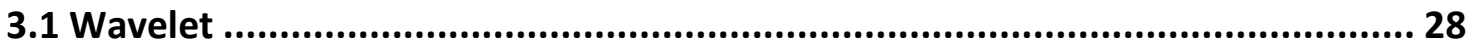

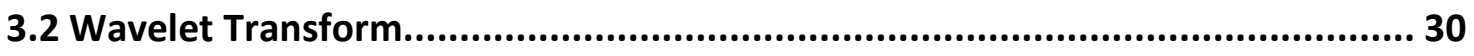

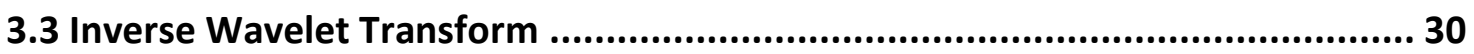

3.4 Discrete Wavelet Transform .......................................................................... 31

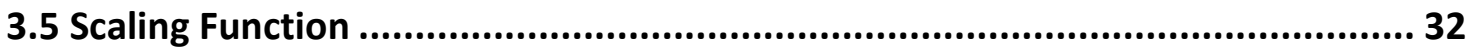

3.6 Sub-band Coding and Multi-Resolution............................................................. 34

3.7 Noise Reduction via Thresholding Method....................................................... 35

3.8 Decomposition and Reconstruction .................................................................. 36

3.9 Implementation ................................................................................................. 41

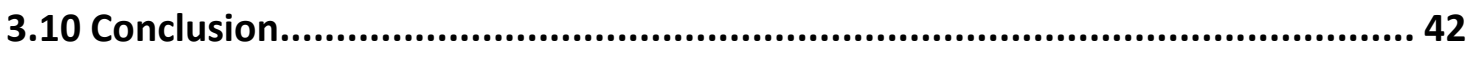

Chapter 4: Wavelet Neural Network .................................................45

4.1 Architectures of WNN ..................................................................................... 46

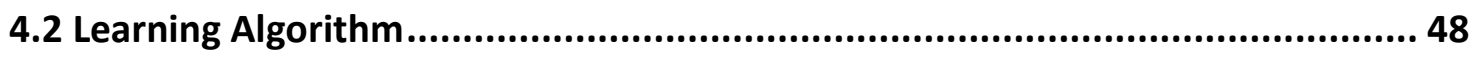

4.2.1 Calculating Gradient ….............................................................................. 49

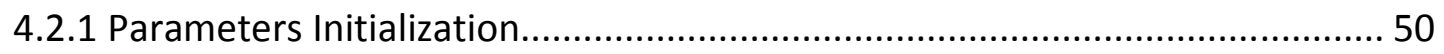




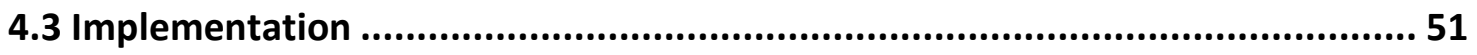

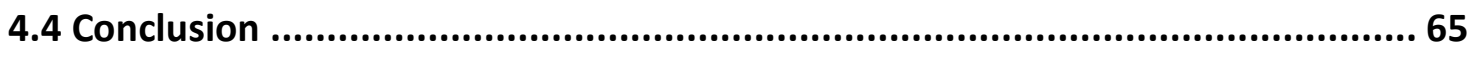

Chapter 5: Learning Algorithms and Design comparison.......................67

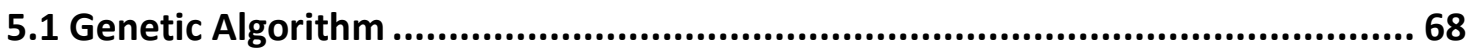

5.2 Particle Swarm Optimization ............................................................................. 70

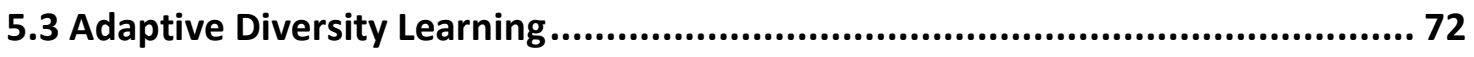

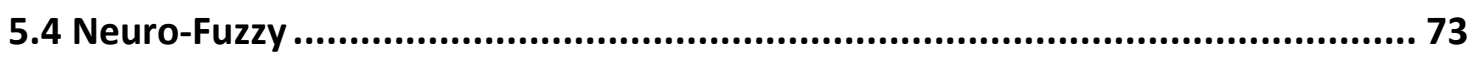

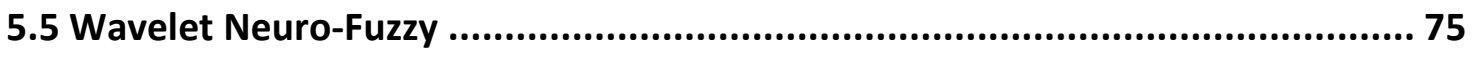

5.6 Local Linear Structure Implemented Wavelet Neural Network ........................... 78

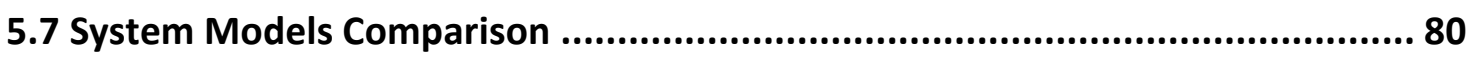

Genetic Wavelet Neural Network (GWNN) ............................................................ 81

GWNN Implementing Multiple Mother Wavelets and Hybrid Gradient Learning

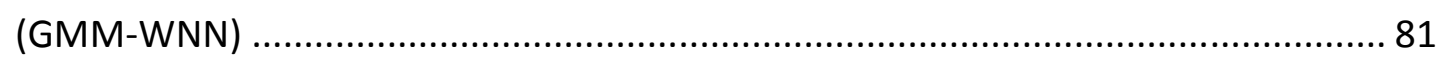

Local Linear Wavelet Neural Network (LLWNN) ……………………………....... 81

Local Linear with Hybrid ADLPSO and Gradient Learning (ADLPSO-LLWNN) ........... 82

Wavelet Neuro-Fuzzy Network (WNFN) .............................................................. 82

Conventional Wavelet Neural Network (WNN) ………………………………..... 82

5.8 ECG Noise Reduction Comparison ...................................................................... 84

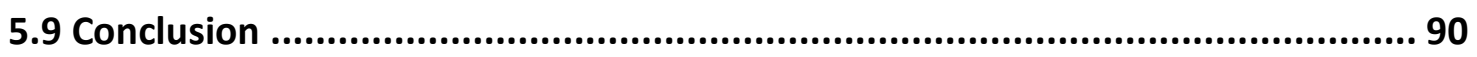

Chapter 6: ECG and Noises Artifact in ECG ........................................92

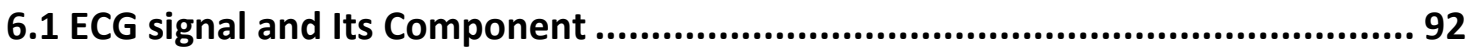

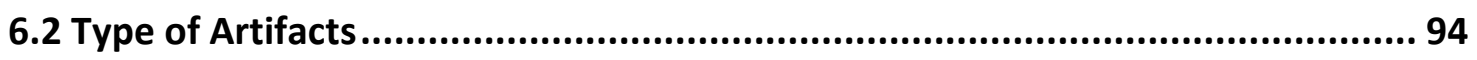

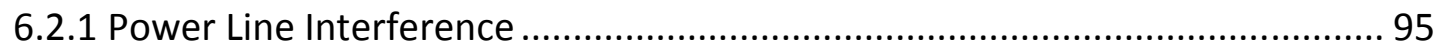

6.2.1 Electrode Contact Noise .......................................................................... 95

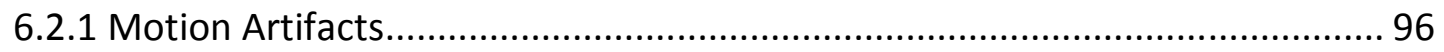

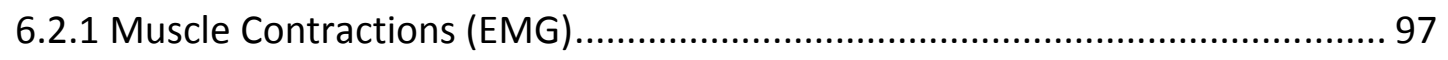

6.2.1 Baseline Drift with Respiration .................................................................. 97

6.2.1 Instrumentation Noise Generated by Electronic Devices ................................ 98

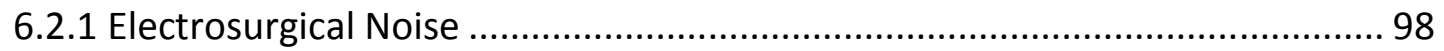




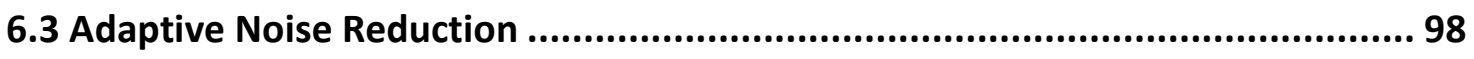

6.3.1 Least Mean Square (LMS) Algorithm ........................................................... 99

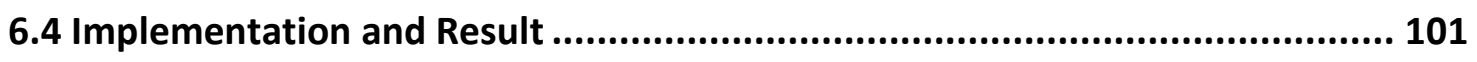

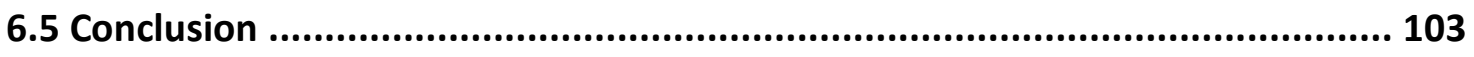

Chapter 7: ECG Noise Reduction......................................................104

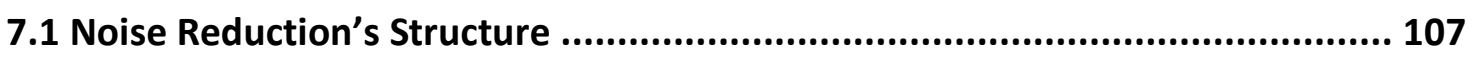

7.1.1 Noise Reduction via Wavelet Decomposition and Neural Networks.............. 107

7.1.1 Discrete Wavelet Transform and Coefficient Thresholding .......................... 110

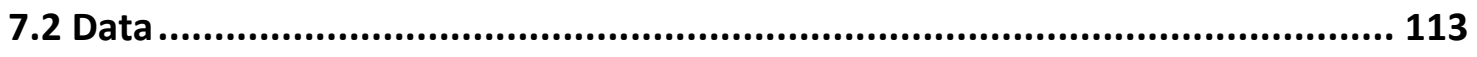

7.2.1 Noises selection .................................................................................... 113

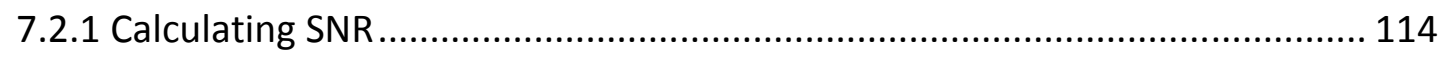

7.3 Simulation Result and Discussion.................................................................. 116

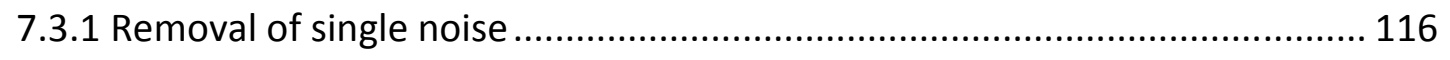

7.3.1 Removal of combined noises................................................................ 120

7.3.1 Effect of DWT and coefficient threshold on the signal .................................. 127

7.3.1 Comparison with Transitional Methods ...................................................... 131

7.3.1 Replacement of DWT with LPF and down-sampling .................................... 133

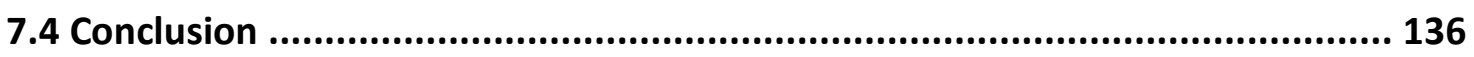

Chapter 8: Discussion and Conclusions ...............................................137

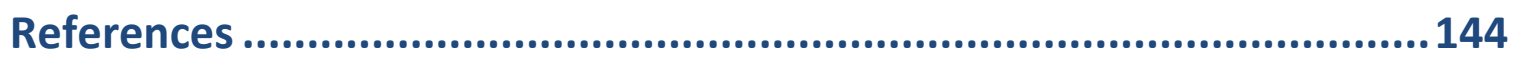

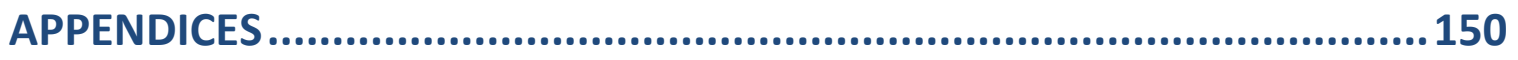

Noise Reduction via Wavelet Decomposition and Neural Network ....................... 150 


\section{LIST OF TABLES}

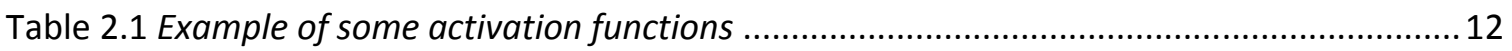

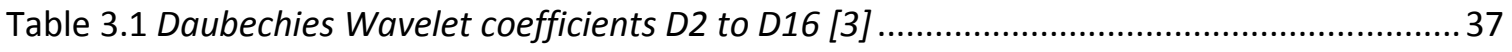

Table 4.1 WNN and ANN parameters for system modeling ......................................................56

Table 4.2 WNN and ANN parameters for noise reduction ........................................................59

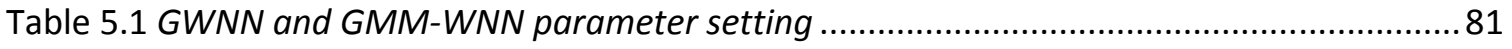

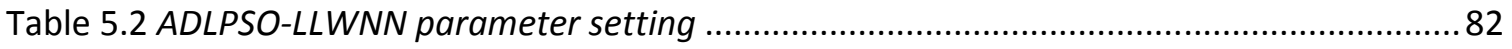

Table 5.3 Simulation result from all models and their parameters comparison........................... 83

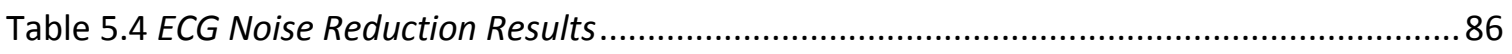

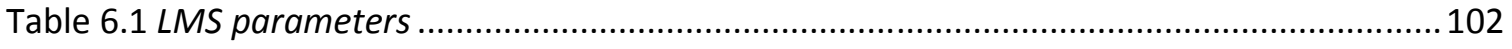

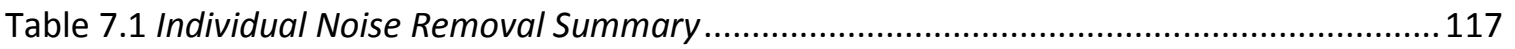

Table 7.2 Performance consistency in term of SNR improvement ............................................ 122

Table 7.3 SNR improvement with larger training network .................................................... 125

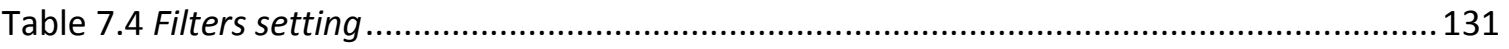

Table 7.5 SNR improvement using LPF and down-sampling .................................................. 134 


\section{LIST OF FIGURES}

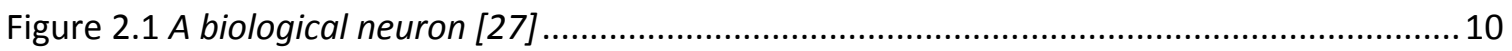

Figure 2.2 A simple mathematical model for a nonlinear neuron. ........................................... 12

Figure 2.3 A very simple neural network with two inputs, one hidden layer of two nodes or units,

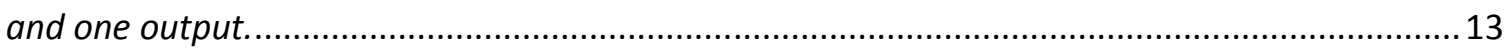

Figure 2.4 Example of activation functions:........................................................................... 14

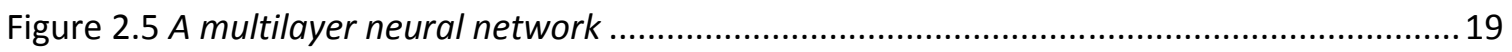

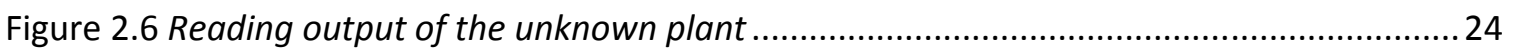

Figure 2.7 Output response of neural network after 200 training iterations of class N(1-8-6-1)

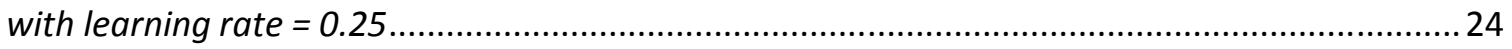

Figure 2.8 Output response of neural network after 1,000 training iterations of class N(1-8-6-1)

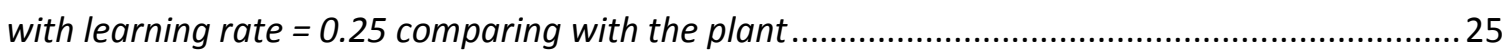

Figure 2.9 Output response of neural network after 2,000 training iterations of class N(1-8-6-1)

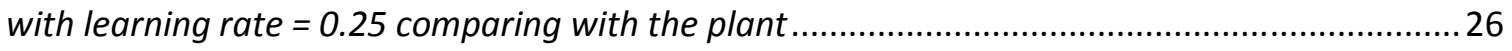

Figure 2.10 Output response of neural network after 2,000 training iterations of class N(1-8-6-1)

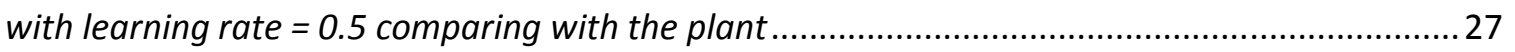

Figure 3.1 Examples of some of the common wavelet: ............................................................29

Figure 3.2 Wavelet spectrum resulting from scaling of mother wavelet......................................33

Figure 3.3 Using scaling spectrum to replace infinite set of wavelet spectrum at the lower

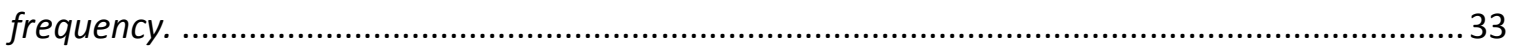


Figure 3.4 Splitting of signal via high-pass filter (HPF) and low-pass filter (LPF) according to figure

3.3. 35

Figure 3.5 Example of Daubechies's frequency spectrum of D4, D8 and D20. .38

Figure 3.6 Schematic of signal filtering of the forward transform to produce wavelet and scaling coefficient with resolution of 3 levels. .40

Figure 3.7 Schematic diagram of the filtering of signal reconstruction from wavelet and scaling coefficients with resolution of 3 levels.

Figure 3.8 DWT using Daubechies wavelet with three levels transformation and 1024 sampling points.

Figure 3.9 The upper plot is the input signal with added-noise. The lower plot is the thresholding signal estimation using Daubechies D16 wavelet transform with three level resolutions. 44

Figure 4.1 A conventional neuron model with activation function 47

Figure 4.2 (a) ANN using wavelets as the activation function. (b) Single neuron modeling wavelet.

Figure 4.3 Basic wavelet neural network structure. .48

Figure 4.4 Wavelet Neural Network with 7 wavelons trained for 10,000 learning iterations........52

Figure 4.5 The original 3-D function to be approximated by WNN. .53

Figure 4.6 An approximated function by WNN after 40,000 iterations. .54

Figure 4.7 Single-layer Artificial Neural Network class N(1-7-1)............................................ 56

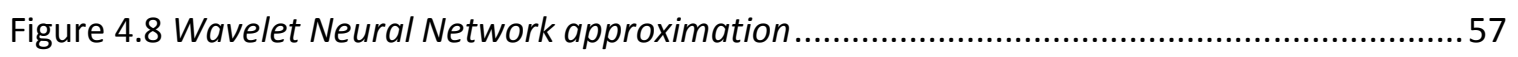

Figure 4.9 Multi-layer Neural Network approximation class N(1-3-3-1) .................................... 57

Figure 4.10 Multi-layer Neural Network approximation class N(1-4-2-1) ...................................58

Figure 4.11 Multi-layer Neural Network approximation class N(1-2-4-1) .................................. 58

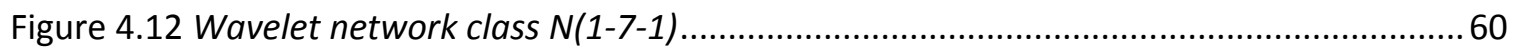




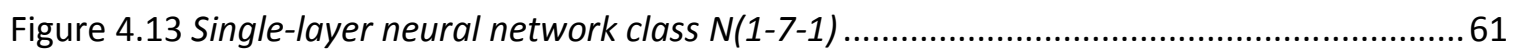

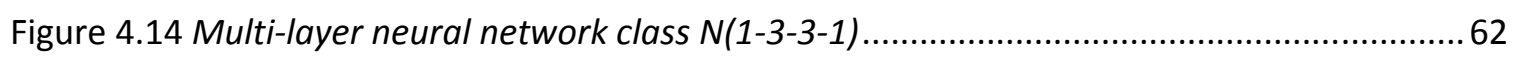

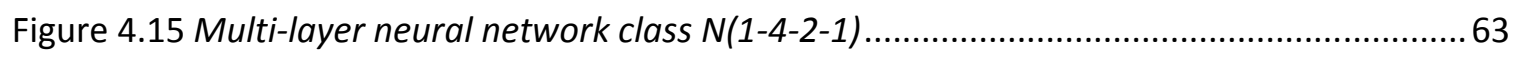

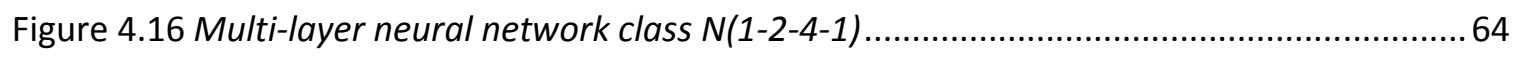

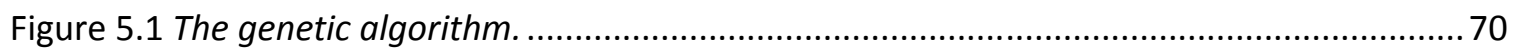

Figure 5.2 Basic Neuro-Fuzzy Network Structure ................................................................. 74

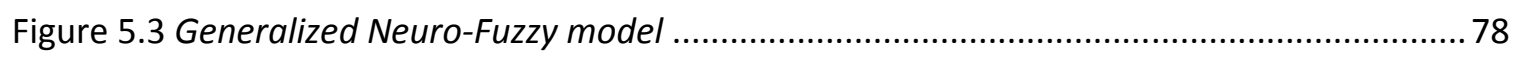

Figure 5.4 Basic wavelet neural network structure ................................................................ 79

Figure 5.5 Objective function error for each model recorded for every iteration .......................... 84

Figure 5.6 Original noise-free ECG (top) and Unfiltered noisy ECG signal (bottom) ..................... 87

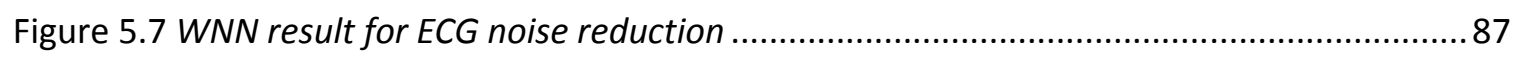

Figure 5.8 GWNN result for ECG noise reduction ............................................................... 88

Figure 5.9 GMM-WNN result for ECG noise reduction........................................................ 88

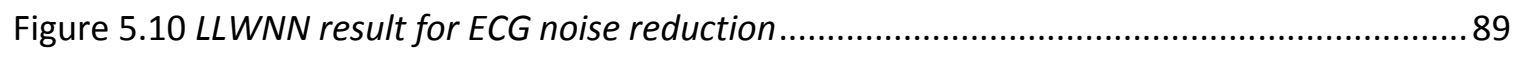

Figure 5.11 ADLPSO-WNN result for ECG noise reduction ..................................................... 89

Figure 5.12 WNFN result for ECG noise reduction ............................................................ 90

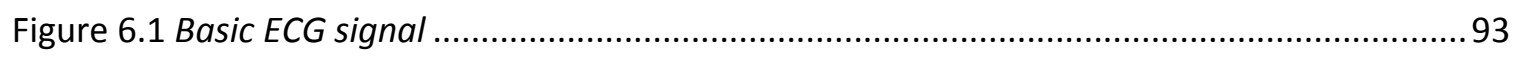

Figure 6.2 The conduction system of the heart. Image is taken from [22] ................................ 94

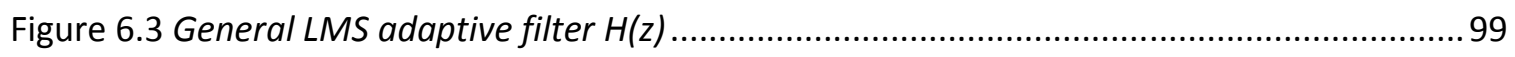

Figure 6.4 Simplified error function with respect to the filter coefficients ................................100

Figure 6.5 LMS result as ECG adaptive power-line noise cancellation ..................................... 102

Figure 7.1 Frequency spectrum analyses of typical ECG signals taken from [30] ...................... 106

Figure 7.2 Frequency spectrum analyses of electrode movement and motion artifact taken from

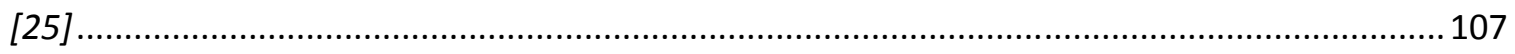




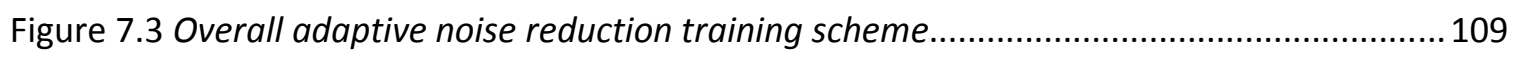

Figure 7.4 SNR gain comparing with number of output of neural network...............................110

Figure 7.5 Wavelet Decomposition and coefficients thresholding .......................................... 112

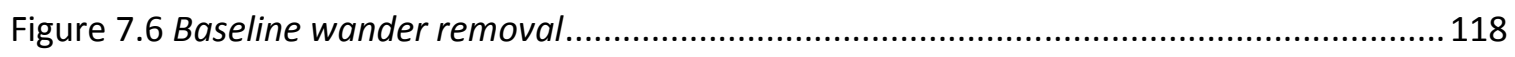

Figure 7.7 Electrode motion artifact removal ..................................................................... 118

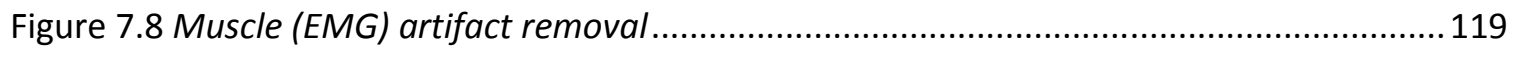

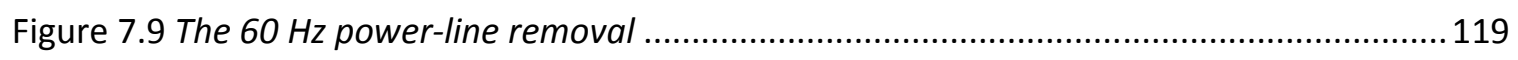

Figure 7.10 Gaussian white noise removal ........................................................................ 120

Figure 7.11 ECG with irregular shape taken from [30]. Record: 119, 208, 233 ........................ 121

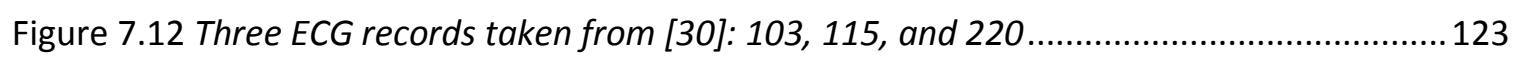

Figure 7.13 Noises combined removal using patient's ECG record 103...................................123

Figure 7.14 Noises combined removal using patient's ECG record 115.................................. 124

Figure 7.15 Noises combined removal using patient's ECG record 220.................................. 124

Figure 7.16 Test result of ECG record 103 from re-training with larger network ....................... 126

Figure 7.17 Test result of ECG record 115 from re-training with larger network ....................... 126

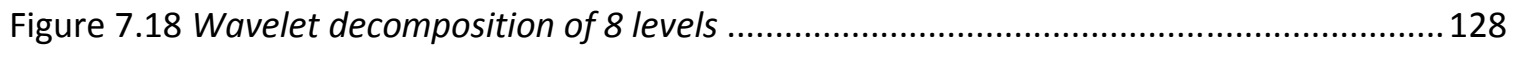

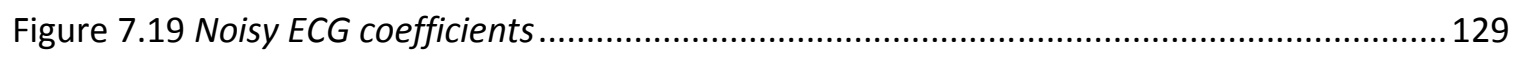

Figure 7.20 Removing coefficients at high frequency ........................................................ 129

Figure 7.21 Comparing noisy ECG signal prior and after the thresholding .............................. 130

Figure 7.22 Frequency analysis of signal prior and after filtering ......................................... 132

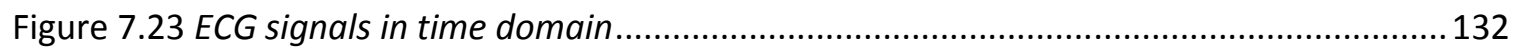

Figure 7.24 Noise reduction comparisons between the proposed method and a similar alternative

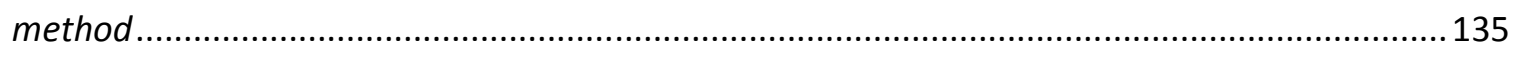

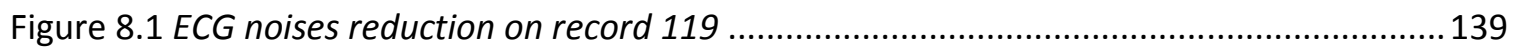




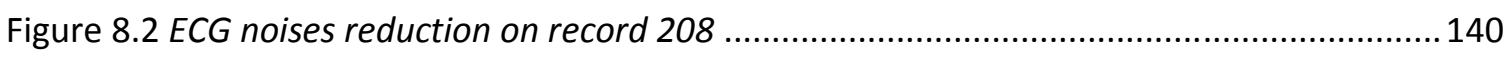

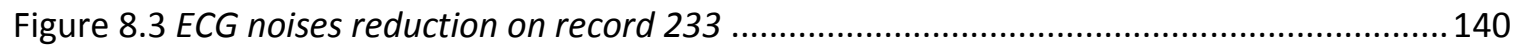




\section{Chapter 1: Introduction}

The electrocardiogram (ECG) signal is a trace of an electrical activity signal generated by rhythmic contractions of the heart and it can be measured by electrodes placed on the body's surface. Clinical data showed that an ECG signal is very effective in detecting heart disease, and there are steady efforts within the research community to develop automated ECG signal classification algorithms. Conventionally, an ECG signal is measured in static conditions, since the appearance of heartbeats varies considerably, not only among patients, but also movement, respiration, and modifications in the electrical characteristics of the body. Moreover, several electrical and mechanical noise components are also added to the signal, making it difficult to extract key features. In general, measured ECG signal data contains white noise, muscle artifact noise, baseline noise, electrode moving artifact noise, and $60 \mathrm{~Hz}$ power line noise [1]. In the effort to remove these noises, there has been little success when employing traditional methods such as linear filers, signal averaging, and their combination.

ECG noise removal is complicated by the fact that the characteristics of almost all biomedical signals vary in time. For instance, ECGs tend to vary quasi-periodically, with each period corresponding to one heart beat; sometimes, even the shape of the ECG beats varies in time [38]. Some of the noise and artifacts are random in nature and have a wide range of frequency content. Because the noise power spectrum resides within 
the frequency range of the cardiac signal, filtration of noise from the ECG signal becomes challenging.

Different techniques have previously been proposed on the removal of the interferences in the ECG signals. Some of them consisted of a median filter for impulsive noise reduction and a linear filter for white noise reduction. For example, an FIR median hybrid filter which was a cascade of linear phase FIR filters and a median filter was proposed in [40]. The Method in [41] presented an IIR median hybrid filter [39]. For baseline removal, the technique of baseline estimation using cubic spline in [45] is proposed. This is a third order approximation where the baseline is estimated by polynomial approximation and then subtracted from the original ECG signal. In [46], the baseline is constructed by linearly interpolating between pre-known isoelectric levels estimated from PR intervals. This is a nonlinear approach and becomes less accurate at low heart rates, where the cubic spline approximation achieved better results. Linear filtering is another method applied to the baseline wander problem. Using this approach, a digital narrow-band linear-phase filter with cut-off frequency of $0.8 \mathrm{~Hz}$ has been suggested in [47]. Another filtering technique using digital and hybrid linear-phase with cut-off frequency of $0.64 \mathrm{~Hz}$ is used in [48]. Time-varying filtering was proposed in [49]. Filter banks with different cut-off frequencies that depend on heart rate and baseline level was implemented. There are numerous problems associated with these filtering methods. First, when the FIR structure is used, the number of coefficients is too high; therefore creating a long impulse response. Secondly, there is an overlap in the spectrums of the baseline and the ECG signals. Thus, removing the baseline spectrum 
will cause distortion in the ECG components. Thirdly, the cut-off frequencies are not copasetic with the American Heart Association (AHA) [50] recommendations for ECG which state that the lower limit must be $0.05 \mathrm{~Hz}$. Removing any frequencies above this will cause distortion in the ST segment as well as QRS complex [51] [44].

Through shielding and clever design of the hardware, the elimination of power-line interference at $60 \mathrm{~Hz}$ has been implemented to minimize the level of the noise. Methods shown in [53] have been developed to increase the actual Common-Mode Rejection Ratio (CMRR) by equalization of the cable shielding and the right leg potentials. This reduces the influence of stray currents through the body, but the efficiency obtained is not sufficient to significantly reduce the interference [52]. Often, a digital filter such as a notch filter is necessary to attenuate the coherent artifact [37].

Some signals, like human respiration, have frequency components sufficiently below the interference frequencies, so that a low-pass filter can isolate the desired components. However, unless the interference has low amplitude, the attenuation of the low-pass filter maybe insufficient to eliminate the interference, especially when the interference frequency is near the cut-off frequency, or when the amplitude of a coherent artifact is larger than the stop band attenuation. In such cases, a higher order low-pass filter may provide sufficient attenuation or, alternatively, a separate notch filter may be cascaded with the low-pass filter. Either solution introduces an additional phase shift or the notch filter contributes a "ringing" component in its time response (e.g., an overshoot and damped oscillation in its step response) [37]. [54] attempted to reduce, to some extent, 
the transient response time by using vector projection to find better initial values for IIR notch filters. Paper [55] proposed a hardware notch filter with adaptive central frequency to follow the power line frequency changes, thus defining a narrower bandwidth. Filters with various $Q$ factors have been tried. However, the resulting signal distortion cannot be correctly assessed because of the reduced scale of the examples provided [55] [52].

A number of approaches have been used to reduce the motion artifact or compensate for motion artifact in the ECG recordings. Abrasion of the skin at the electrode site [57] is the standard method for reducing motion artifact. [58] and [59] proposed refined skin puncture as a method for reducing motion artifact. Though commonly used for reducing motion artifact, skin abrasion or puncture has a number of practical drawbacks. It is hard to tell just how much skin abrasion is required [57]: too much abrasion can lead to skin irritation; too little abrasion will not significantly reduce the motion artifact. Adequate preparation of all electrode sites in a 12-lead stress recording takes considerable time. Consequently, technicians may skip skin abrasion simply because it represents an added complication and increases patient discomfort. Over the course of longer recordings, such as Holter's recordings, skin abrasions can heal, increasing the possibility of motion artifact.

A novel technique for the detection of motion artifact using electrode/skin impedance measurements was proposed in [60]. The researchers injected a $20 \mathrm{kHz}$ current at the electrode site and monitored the resulting $20 \mathrm{kHz}$ voltage to estimate $20 \mathrm{kHz}$ 
skin/electrode impedance at the ECG recording site. The researchers assumed that the impedance signal would be correlated with the resulting motion artifact. They used significant impedance variation to indicate the presence of motion artifact. The group also investigated the use of an impedance signal to actually remove motion artifact from the original ECG signal by the correlation techniques, but, though they were able to reduce motion artifact in some cases, they were not able to reliably remove motion artifact [56]. Many digital ECG filtering algorithms have also been developed. But as motion artifacts' bandwidth overlaps with the ECG's [62], linear filtering techniques showed to be inefficacious [63] [61].

And like Electrode motion artifact, the spectra of muscle (EMG) artifact and ECG signals are also overlapped. Hence, simple elimination by using basic filters is also not an effective solution [64].

In general, there have been many efforts in ECG noise reduction. There are many methods that had shown promising results. Still, there are some methods that need improvement. Due to the fact that there is no formal method which can separate nonstationary signals with over-lapped frequencies spectrum, success from implementation using the traditional methods cannot be expected. Linear filter approaches are defined by fixed frequency spectrum which may result in signal distortion as characteristic of ECG vary from patient to patient. There are also preventative methods but they often require additional hardware and various setups that are time-consuming and pose discomfort for many patients. Even so, interferences are only attenuate in amplitude 
and are still visible. In order to be successful, any noise removal procedure for biomedical signals must be adaptive. That is, it must track the changing signal characteristics.

There are number of adaptive system readily available to choose from. They are Wiener, Least mean squares (LMS), Recursive least squares (RLS), Gauss-Newton Algorithm, and Linear predictive coding (LPC), etc. However, in this paper, an artificial neural network (ANN) is chosen as the proposed technique. Comparing to other algorithms, ANN provides better adaptive, learning, and recognizing capability for non-stationary signal. As the interference in the cardiac signal often destroys the signal completely, the filter is required to perform signal reconstruction from its memory. Therefore, aside from noise reduction duty, the filter needs to perform signal recognition and generalization. Based on this criterion, ANN seems to be the best candidate.

Neural networks have demonstrated an effective response for the dynamics systems whose behavior is nonlinear [6]. Its internal mechanisms are developed to emulate the human brain that is powerful, flexible, and efficient. The characteristics of sigmoid function and simple learning algorithms like back-propagation can be incorporated to the system that allow neural network to discover solutions automatically through supervised learning. Using this simple learning algorithm, neural networks can extract the input's and output's regularity based on the observation of learning samples. Thus, avoiding the dependency for details of the signals studied. However, conventional neural networks can process signals only by its finest input resolutions. Hence, better 
extraction of key component features prior to the processing of neural network can reduce the computational burden on neural network and drastically improve output results.

Recently, the subject of wavelet decomposition analysis has attracted much attention from both mathematicians and engineers alike. Wavelets have been applied successfully to multi-scale analysis and synthesis, time-frequency signal analysis in signal processing, function approximation, and approximation in solving partial differential equations, etc [16]. Wavelets are well suited to depicted functions with local nonlinearities and fast variations because of their intrinsic properties of finite support and self-similarity. The introduction of wavelet decomposition provides a new tool for signal analysis. It produces a good local representation of the signal in both the time and the frequency domains.

Inspired by both the neural network and wavelet decomposition, Wavelet Neural Network (WNN) was introduced in [3]. This has led to rapid development of neural network models integrated with wavelets. Most researchers use wavelets as a basis function that allows for hierarchical, multi-resolution learning of input-output maps of data. The proposed method presented in this paper does not involve such hybrid combination. Rather, the wavelet technique is used as a feature extraction, aiding the neural network in capturing useful information on the dynamic of complex time series. However, for the sake of completeness, a Wavelet Neural Network is also introduced. 
Similar techniques have been implemented in [65], [66], [67], and [68]. Paper [65] utilized the combination of several dynamical recurrent neural networks capturing the dynamics of several multi-resolution versions of a data signal in prediction problems. Similar to [65], paper [66] also implemented the methods for prediction problems but used simple feed-forward neural networks. Paper [67] worked on ECG beat detection and classification, while paper [68] worked on noise suppression. Unlike other papers, [68] chose to train a neural network with wavelet coefficients as references rather than the actual final output. As a result, the authors in [68] also included an inverse wavelet transform at the output of neural network for signal reconstruction.

This paper is organized in 8 chapters. Chapter 2 and 3 laid the groundwork for the chapters that follow by providing literature reviews on neural network and wavelet decomposition. Chapter 4 introduces the concept of the generalized wavelet neural network and its capability in comparison to the conventional neural network. Further improvements and variation on the designs and methodologies over the generalized wavelet neural network proposed by various researchers are presented and compared in chapter 5 . Chapter 6 provides a basic understanding of the ECG signal as well as a brief introduction to some of the most common noise sources associated with ECG signals. Finally, an ECG noise reduction scheme implementing wavelet decomposition and neural network together with discussion and possible improvement on future work are presented in chapters 7 and 8. 


\section{Chapter 2: Artificial Neural Network}

\subsection{About Neural Networks}

The work of Artificial Neural Networks (ANN) or "neural networks" has been motivated by the human cognitive process that the human brain computes in an entirely different way from the conventional digital computer. The brain is a highly complex, nonlinear, and parallel in computing. It consists of interconnected processing elements called "neurons," whose principal function is the collection, processing, and dissemination of electrical signals, that work together to produce appropriate output response. The brain's information-processing capacity is thought to emerge primarily from networks of such neurons. For this reason, the model of artificial neural network was created. The network has a series of external inputs and outputs which take or supply information to the surrounding environment. Figure 2.1 provides the basics structure of biological neurons which made up of:

- Synapses - point of connection between two neurons

- Dendrite - integration and transfer of incoming signal from synapses

- Soma - determine action potential, induction and a threshold condition

- Axon - transmission line carrying output signal (action potential) to the synapses of other neurons 


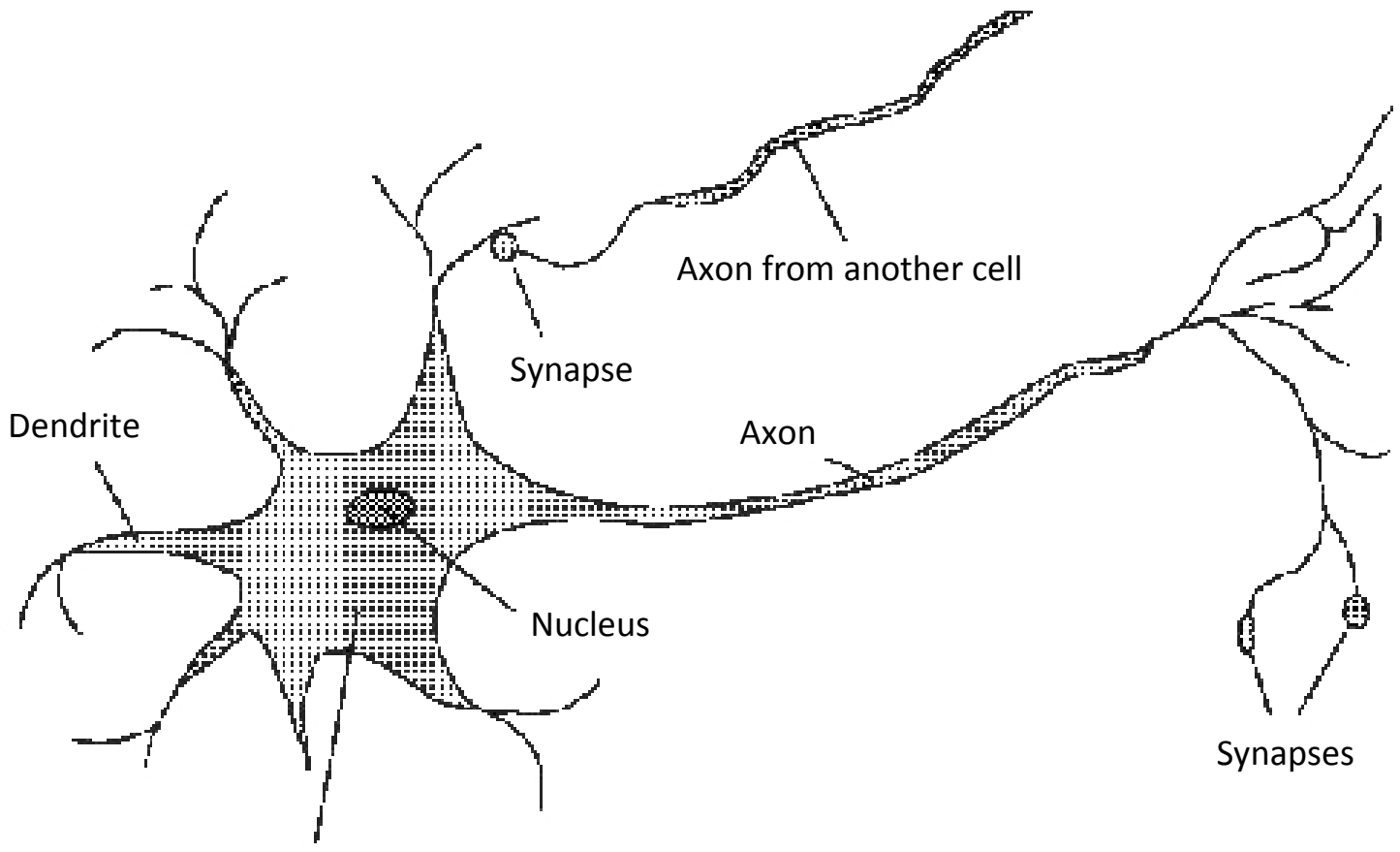

Cell Body or Soma

Figure 2.1 A biological neuron [27]

Inter-neuron connections are called synapses which have associated synaptic weights.

The weights are used to store knowledge which is acquired from the environment. Learning is achieved by adjusting the weights in accordance with a learning algorithm. It is also possible for neurons to evolve by modifying their own topology, which is motivated by the fact that neurons in the human brain can die and new synapses can grow. In its most general form, an artificial neural network is a machine that is designed to model the way in which the brain performs a particular task or function of interest. 


\subsection{Modeling of a Neuron}

Neural networks are composed of nodes/units (see figure 2.2) connected by directed links. Each link has a numeric weight associated with it, which determines the strength and sign of the connection.

Activation function, denoted $\phi($.$) , defines the output of the neuron in terms of the local$ field $v$. Three common choices of activation functions are: Threshold (step) Function, Piecewise-Linear (ramp) Function, and Sigmoid (logistic) Function. Their characteristics are shown in figure 2.4. Other type of activation functions include: Identify, Hyperbolic, Negative exponential, Softmax, Unit sum, Square root, and Sine Function. See table 2.1 for the functions' definition and operational output range [4]. The selection of each particular activation function depends highly on application and personal reference. For common practice, the hyperbolic is usually a function of choice as it has an advantage of being differentiable, which is very significant for the weight-learning algorithm and will be described in a later section. Often, the hyperbolic performs better than the logistic function because of its symmetry.

Mathematically the output of the neuron can be described by the following equations:

$$
\begin{aligned}
& v=\left(\sum_{i=1}^{n} w_{i} x_{i}\right)-\theta \\
& v=\widetilde{w} \cdot \tilde{x}-\theta \text { where }\left\{\begin{array}{l}
\widetilde{w}=\left(\theta, w_{1}, w_{2}, \ldots w_{n}\right) \\
\widetilde{x}=\left(-1, x_{1}, x_{2}, \ldots x_{n}\right) \\
y=\phi(v)
\end{array}\right.
\end{aligned}
$$


Notice that a bias weight is connected to a fixed input -1 . This is because bias weight set the actual threshold for the unit, in the sense that the unit is activated when the weighted sum of "real" inputs $\sum_{i}^{n} w_{i} x_{i}$ (i.e., excluding the bias input) exceeds $\theta$.

Table 2.1 Example of some activation functions

\begin{tabular}{|c|c|c|}
\hline Function & Definition & Range \\
\hline Identify & $x$ & $(-\infty,+\infty)$ \\
\hline \multirow{2}{*}{ Logistic } & 1 & \multirow{2}{*}{$(0,+1)$} \\
\hline & $\overline{1-e^{-x}}$ & \\
\hline \multirow{2}{*}{ Hyperbolic } & $1-e^{-x}$ & \multirow{2}{*}{$(-1,+1)$} \\
\hline & $\overline{1+e^{-x}}$ & \\
\hline $\begin{array}{l}\text { Negative } \\
\text { Exponential }\end{array}$ & $e^{-x}$ & $(0,+\infty)$ \\
\hline \multirow{2}{*}{ Softmax } & $e^{x}$ & \multirow{2}{*}{$(0,+1)$} \\
\hline & $\sum_{i} e^{x_{i}}$ & \\
\hline \multirow[t]{2}{*}{ Unit Sum } & $e^{x}$ & \multirow[t]{2}{*}{$(0,+1)$} \\
\hline & $\sum_{i} x_{i}$ & \\
\hline Square Root & $\sqrt{x}$ & $(0,+1)$ \\
\hline Sine & $\sin (x)$ & $(0,+\infty)$ \\
\hline \multirow[b]{2}{*}{ Ramp } & $(-1, x \leq-1)$ & \multirow[b]{2}{*}[-1,+1]{} \\
\hline & $\left\{\begin{array}{c}x,-1<x<+1 \\
+1, x \geq+1\end{array}\right\}$ & \\
\hline Step & $\left\{\begin{array}{c}0, x<0 \\
+1, x \geq 0\end{array}\right\}$ & {$[0,+1]$} \\
\hline
\end{tabular}

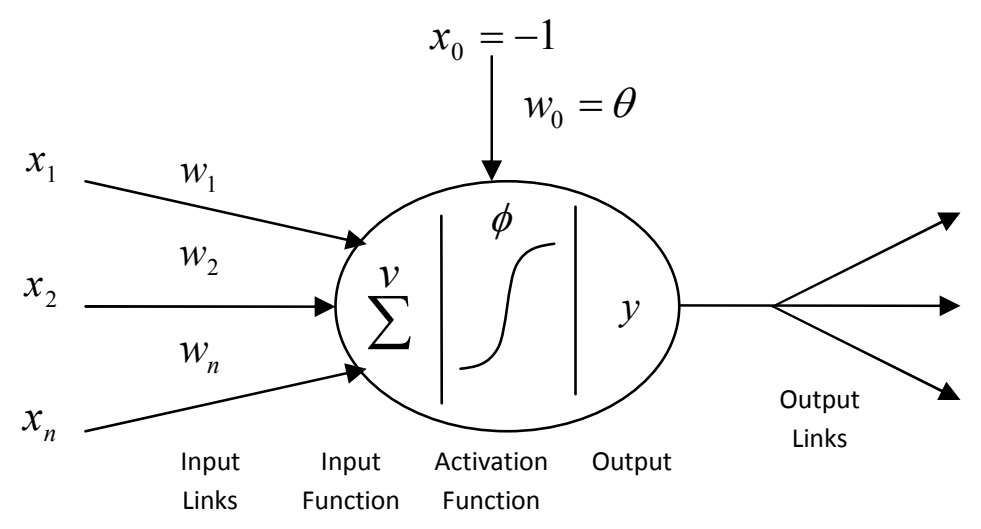

Figure 2.2 A simple mathematical model for a nonlinear neuron.

The unit's output activation is $y=\phi\left(\sum_{i=0}^{n} w_{i} x_{i}\right)$, where $y$ is the output of the activation function and $w_{i}$ is the weight on the link from previous unit. 


\subsection{Architectures of Neural Networks}

There are two main categories of neural network structures: acyclic or feed-forward networks and cyclic or recurrent networks. A feed-forward network represents a function of its current input; thus, it has no internal state other than the weights themselves. A recurrent network, on the other hand, feeds its outputs back into its own inputs. This means that the activation levels of the network form a dynamical system that may reach a stable state or exhibit oscillations or even chaotic behavior. Moreover, the response of the network to a given input depends on its initial state, which may depend on previous inputs. Hence, recurrent networks (unlike feed-forward networks) can support short-term memory. This makes them more interesting as models of the brain, but also more difficult to understand. For this paper, the focus and implementation will be entirely concentrated on feed-forward networks. Figure 2.3 displays the simple form of a feed-forward network which has two input units, two hidden units, and an output unit. Note that the bias terms are omitted for the sake of simplicity. The subscript of 1 and 3 for $w_{1,3}$ identifies the weight connection between unit 1 or input node 1 and hidden unit 3.

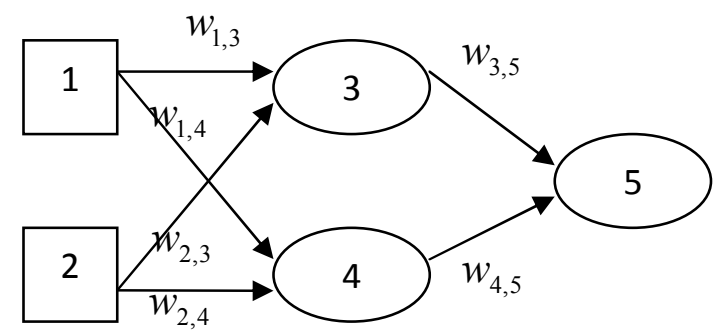

Figure 2.3 A very simple neural network with two inputs, one hidden layer of two nodes or units, and one output. 


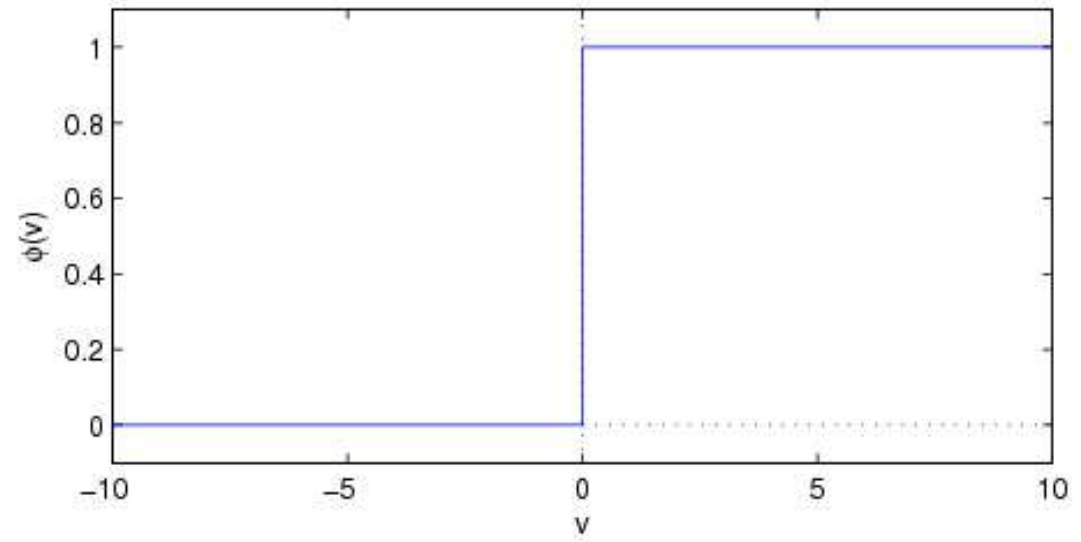

(a) Threshold (Step) Function

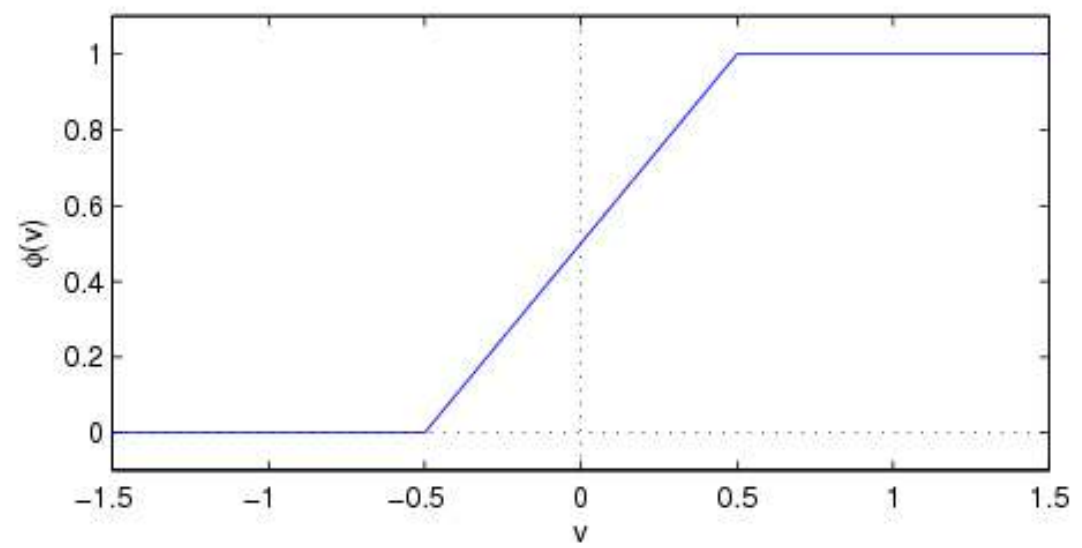

(b) Piecewise-Linear (Ramp) Function

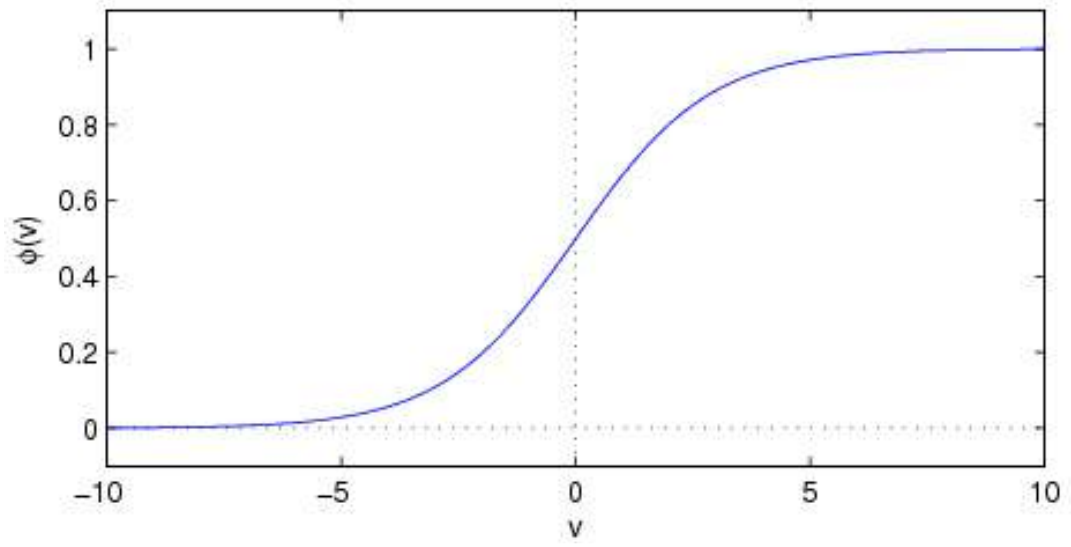

(c) Sigmoid (Logistic) Function

Figure 2.4 Example of activation functions:

(a) The threshold activation function, which outputs 1 when the input is positive and 0 otherwise. (b) The piecewise-linear function defines the amplification factor inside the linear region to be unity. (c) The sigmoid function: $1 /\left(1+e^{-x}\right)$. 
Given an input vector $\tilde{x}=\left(x_{1}, x_{2}\right)$, the activation of the input units are set to $\left(y_{1}, y_{2}\right)=\left(x_{1}, x_{2}\right)$ and the network computes:

$$
\begin{gathered}
y_{5}=\phi\left(w_{3,5} y_{3}+w_{4,5} y_{4}\right) \\
=\phi\left(w_{3,5} \phi\left(w_{1,3} y_{1}+w_{2,3} y_{2}\right)+w_{4,5} \phi\left(w_{1,4} y_{1}\right.\right. \\
\left.\left.\quad+w_{2,4} y_{2}\right)\right)
\end{gathered}
$$

where $y_{1}$ is output of node 1 , and $y_{2}$ is output of node 2, etc. Equation (2.3) is rewritten by expressing the output of each hidden unit as a function of its inputs. The equation above has shown that output of the network as a whole, $y_{5}$, is a function of the network's inputs. Furthermore, the weights in the network act as parameters of this function. By adjusting the weights, this affects the function that the network represents. This is how learning occurs in the neural networks.

\subsection{Back Propagation}

Back propagation is a supervised learning technique used for training artificial neural networks. In the training process of the neural network, the network learns a predefined set of input-output example pairs. After the output $\tilde{y}$ at the output layer is generated from the input $\tilde{x}$ applied to the input layer, the output pattern is then compared to the desired output, and error signal is computed for each output unit. Error signals are then transmitted backward from the output layer to each node in the intermediate layer that contributes directly to the output. However, each unit in the intermediate layer receives only a portion of the total error signal, based roughly on the 
relation contribution the unit made to the original output. This process repeats, layer by layer, until each node in the network has received an error signal that describes its relative contribution to the total error. Based on the error signal received, connection weights are then updated by each unit to cause the network to converge toward a state that allows all the training patterns to be encoded. With enough training iterations, which may vary from application to application, the weights of the network will adjust to inhabit the feature that they are trained to recognize.

\subsubsection{Weight Adjustment for Output Layer}

The idea behind the algorithm is to adjust the weight of the network to minimize some measure of the error in the training set. The "classical" measure of error is the sum of squared errors. The error at a single output unit is defined to be $\varepsilon=\left(y_{d}-y_{k}\right)$, where the desired output is $y_{d}$ and the actual output is $y_{k}$. The squared error for a single training example is written as:

$$
E=\frac{1}{2} \varepsilon^{2}
$$

Using gradient descent to reduce the squared error by calculating the partial derivative of $E$ with respect to each weight:

$$
\begin{aligned}
\frac{\partial E}{\partial w_{k}} & =\varepsilon \times \frac{\partial \varepsilon}{\partial w_{k}} \\
& =\varepsilon \times \frac{\partial}{\partial w_{k}}\left[y_{d}-\phi\left(\sum_{k=0}^{n} w_{k} x_{k}-\theta\right)\right] \\
& =-\varepsilon \times \phi^{\prime}(v) \times x_{k}
\end{aligned}
$$


where $\phi^{\prime}($.$) is the derivative of the activation function. For the hyperbolic function$ chosen to be implemented at the end of this chapter, this derivative is found to be: $\phi^{\prime}()=.\frac{1}{2}\left(1-\phi^{2}\right)$. In the gradient descent algorithm, where the error is reduced, the weight is updated as follows:

$$
w_{k}(t+1)=w_{k}+\eta \times \varepsilon \times \phi^{\prime}(v) \times x_{k}
$$

where $\eta$ is the learning rate. It can be thought of as step size. Keep in mind that while a large $\eta$ enables the network to move toward the optimal state quicker, it also may not be able to converge due to the problem of over-stepping. Hence, choosing the right $\eta$ determines how well the network can learn. Intuitively, equation (2.6) makes a lot of sense. If the error $\varepsilon=\left(y_{d}-y_{k}\right)$ is positive, then the network output is too small and so the weight are increased for the positive inputs and decreased for the negative inputs. The opposite happens when the error is negative.

\subsubsection{Weight Adjustment for Hidden Layers}

Weight adjustment of the hidden layer is different from that of the output layer. This is because output layer error is found by comparing the actually output $y_{k}$ with desired output $y_{d}$. However, there is no method to determine the desired or correct output for each hidden unit. Intuitively, the total error, $\varepsilon$, must somehow be related to the output values on the hidden layer. Hence, the equation for back-propagation shall be derived from the output error, summing over the nodes at the output layer. 


$$
E=\frac{1}{2} \sum_{k}\left(y_{d, k}-y_{k}\right)^{2}
$$

This time, the calculation of $E$ is done with respect to the hidden layer weights $w_{i, j}$. Refer to figure 2.5 for variables' name and their subscripts. To obtain the gradient with respect to the weights $w_{i, j}$ connecting the input layer to the hidden layer, the entire summation over $k$ must be kept because each output value $y_{k}$ may be affected by changes in $w_{i, j}$.

$$
\begin{aligned}
\frac{\partial E}{\partial w_{i, j}} & =\frac{1}{2} \sum_{k} \frac{\partial}{\partial w_{i, j}}\left(y_{d, k}-y_{k}\right)^{2} \\
& =-\sum_{k}\left(y_{d, k}-y_{k}\right) \frac{\partial y_{k}}{\partial w_{i, j}} \\
& =-\sum_{k}\left(y_{d, k}-y_{k}\right) \frac{\partial \phi\left(v_{k}\right)}{\partial w_{i, j}} \\
& =-\sum_{k}\left(y_{d, k}-y_{k}\right) \phi^{\prime}\left(v_{k}\right) \frac{\partial v_{k}}{\partial w_{i, j}} \\
& =-\sum_{k} \delta_{k} \frac{\partial}{\partial w_{i, j}}\left(\sum_{j} w_{j, k} y_{j}\right) \text { where } \delta_{k}=\left(y_{d, k}-y_{k}\right) \times \phi^{\prime}\left(v_{i}\right) \\
& =-\sum_{k} \delta_{k} w_{j, k} \frac{\partial y_{j}}{\partial w_{i, j}} \\
& =-\sum_{k} \delta_{k} w_{j, k} \frac{\partial \phi\left(v_{j}\right)}{\partial w_{i, j}} \\
& =-\sum_{k} \delta_{k} w_{j, k} \phi^{\prime}\left(v_{j}\right) \frac{\partial v_{j}}{\partial w_{i, j}} \\
& =-\sum_{k} \delta_{k} w_{j, k} \phi^{\prime}\left(v_{j}\right) \frac{\partial}{\partial w_{i, j}}\left(\sum_{i} w_{i, j} y_{i}\right) \\
& =-\sum_{k} \delta_{k} w_{j, k} \phi^{\prime}\left(v_{j}\right) y_{i} \\
& =-y_{i} \delta_{j}
\end{aligned}
$$


Input units Hidden units Output unit

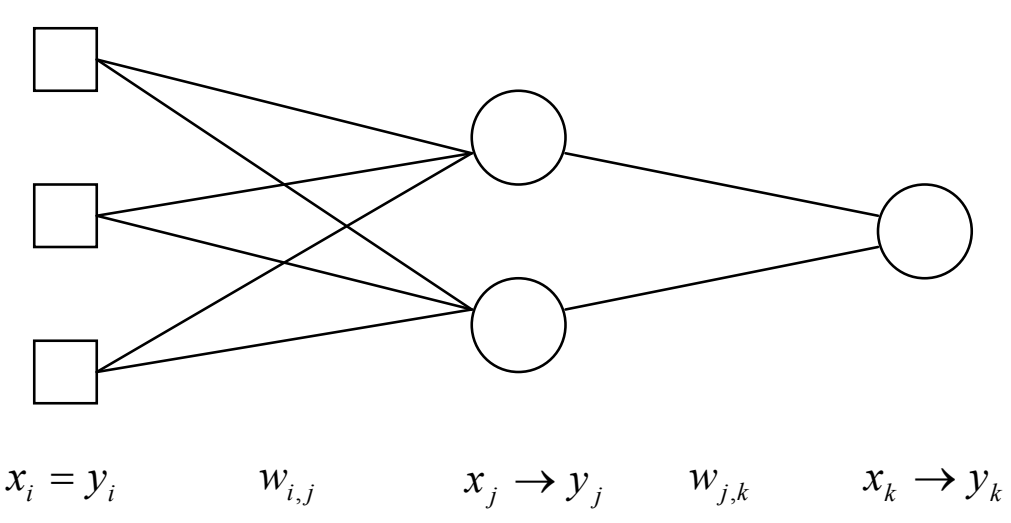

Figure 2.5 A multilayer neural network

where $x$ is indicated as the input value and $y$ is the output with $w$ as weights. Subscript $i, j$, and $k$ represent layers of the network.

From the derivation of the equation 2.8 , the weight update equation for the hidden layer can be expressed as:

$$
w_{i, j}(t+1)=w_{i, j}+\eta y_{i} \delta_{j}
$$

where $\delta_{j}=\phi^{\prime}\left(v_{j}\right) \sum_{k} \delta_{k} w_{j, k}, \phi^{\prime}()=.\frac{1}{2}\left(1-\phi^{2}\right)$, and $\eta$ is, again, learning rate.

\subsubsection{Training Procedure}

The following summarize the training procedure for neural networks:

1. Apply input vector to the input units.

2. Calculate the net-input values to the hidden layer units.

3. Calculate the output from the hidden layer.

4. Move to the output layer. Calculate the net-input values to each unit. 
5. Calculate the output.

6. Calculate the error terms from the output units.

7. Calculate the error terms for the hidden units. Note that the error terms on the hidden units are calculated before the connection weights to the output-layer units have been updated.

8. Update weights on the output layer.

9. Update weights on the hidden layer. The order of the weight updates on an individual layer is not important. One must be sure to calculate the error term.

The error quantity is the measure of how well the network is learning. When the error is acceptably small, training can be discontinued.

\subsection{Simulations and Result}

In this section, a neural network will be demonstrated in an application of system modeling. The network is trained to model the unknown plant of the form:

$$
f(u)=0.6 \sin (\pi u)+0.3 \sin (3 \pi u)+0.1 \sin (5 \pi u)
$$

The plant is governed by the difference equation:

$$
y_{p}(k+1)=0.3 y_{p}(k)+0.6 y_{p}(k-1)+f[u(k)]
$$

In order to identify the plant, $f($.$) is replaced by the neural network:$

$$
\hat{y}_{p}(k+1)=0.3 y_{p}(k)+0.6 y_{p}(k-1)+N[u(k)]
$$

And the input to the plant and the model is a sinusoid function: 


$$
u(k)=\sin \left(\frac{2 \pi k}{250}\right)
$$

The target system to be identified is clearly non-linear and was used and studied in [6] on the convergence of neural network. The system is known as series-parallel model [6].

The simulation was conducted under different training iterations as well as different learning rates $\eta$. Figure 2.6 displays the output of the unknown plant, where the neural network is to be trained to be modeled after. Figure 2.7 shows the response of the neural network after 200 times training iterations with $\eta=0.25$ and the class is $N_{1,8,6,1}$. Here, the model seems to follow the basic characteristic but failed to match up at the higher frequencies. The reason could be because the network required higher training. Hence, the training iteration of 1,000 is deployed using the same setup as before. Figure 2.8 shows the result that higher training iterations provide slightly better matching to the plant but still fail to match on the high frequency parts. Nevertheless, the output of the difference equation comparing the plant and the neural network model is very close. In figure 2.9, the training iteration is increased to 2,000 in hope that the output will provide even better match to the plant.

Figure 2.9(a) displays an unexpected result. Despite the fact that the training iteration number is double that of the one in figure $2.8(\mathrm{a})$, the output response is almost identical between the two. The reason for this could be that during the learning process, the neural network might have fell or trapped at the local minima before reaching the most optimal global solution. Typically, this problem can be overcome by providing higher learning rate. In the next experiment, the learning rate is increased from $\eta=$ 
0.25 to $\eta=0.5$. Increasing the learning rate may help to avoid settling into a local minimum. Higher learning rates allow the network to potentially skip through local minima. Conversely, setting the learning rate too high may result in training which is highly unstable and thus may not achieve even a local minimum.

Figure 2.10 is the result of the neural network deployed with 2,000 training iterations with $\eta=0.5$ and belonging to the class $N_{1,8,6,1}$. The output of the neural network model and the plant matches well with one another for the most part. The problem occurred with figure $2.8(a)$ was indeed caused by the learning rate that is too low.

\subsection{Conclusion}

Neural networks introduced in this chapter can be used for black-box identification of general non-linear systems; their ability is demonstrated. With the right setting parameters, the network is able to successfully model a given plant. Aside from its simple structure and easy to implement, a neural network does not require a prior mathematical model. Its learning algorithm is used to adjust, sequentially by trial and error during the learning phase, the synaptic-weight of the neurons to minimize the error at the output. Despite all these, neural network also has some limitation.

The issue of "cure of dimensionality" continues to be the main issue in neural networks research. Higher dimensional problem means that neural networks need to increase the number of its neurons. Additionally, there is also a problem concerning the relationship 
between data and network's size. Currently, there has not been a formal method in determining network's size in relationship with its data. The Akaike's final prediction error criterion [29] can be used to determine a simple structure such as a single hidden layer network but cannot provide approximation for multiple hidden layer structures. The most common way of finding a "good" network size is simply by testing it out. This is also hold truth for the learning rate.

Nevertheless, neural networks provide a simple and easy way to model a plant through the observation of input-output relationships which is a big advantage over other methods that require additional mathematical information and long series expansions. 


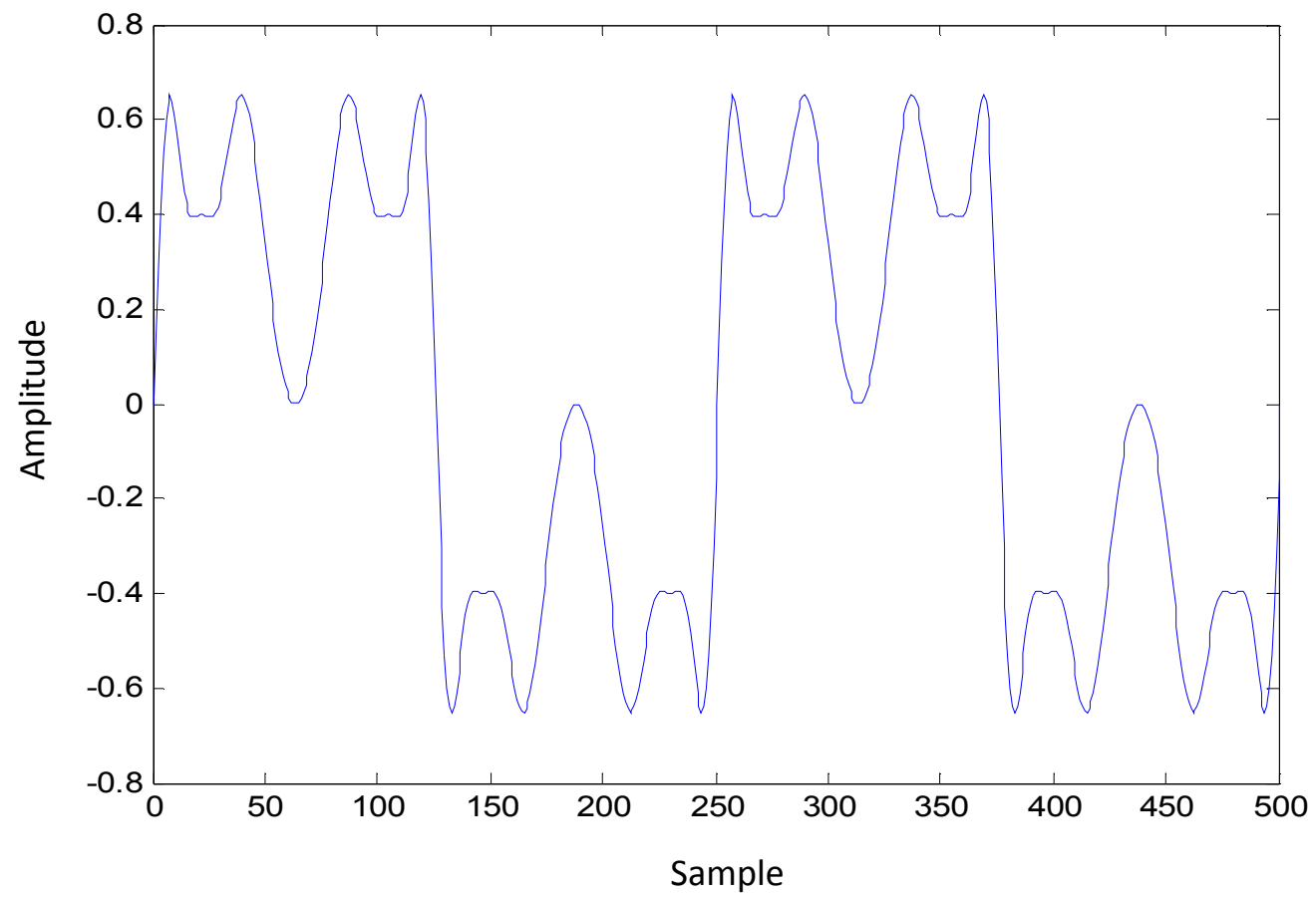

Figure 2.6 Reading output of the unknown plant

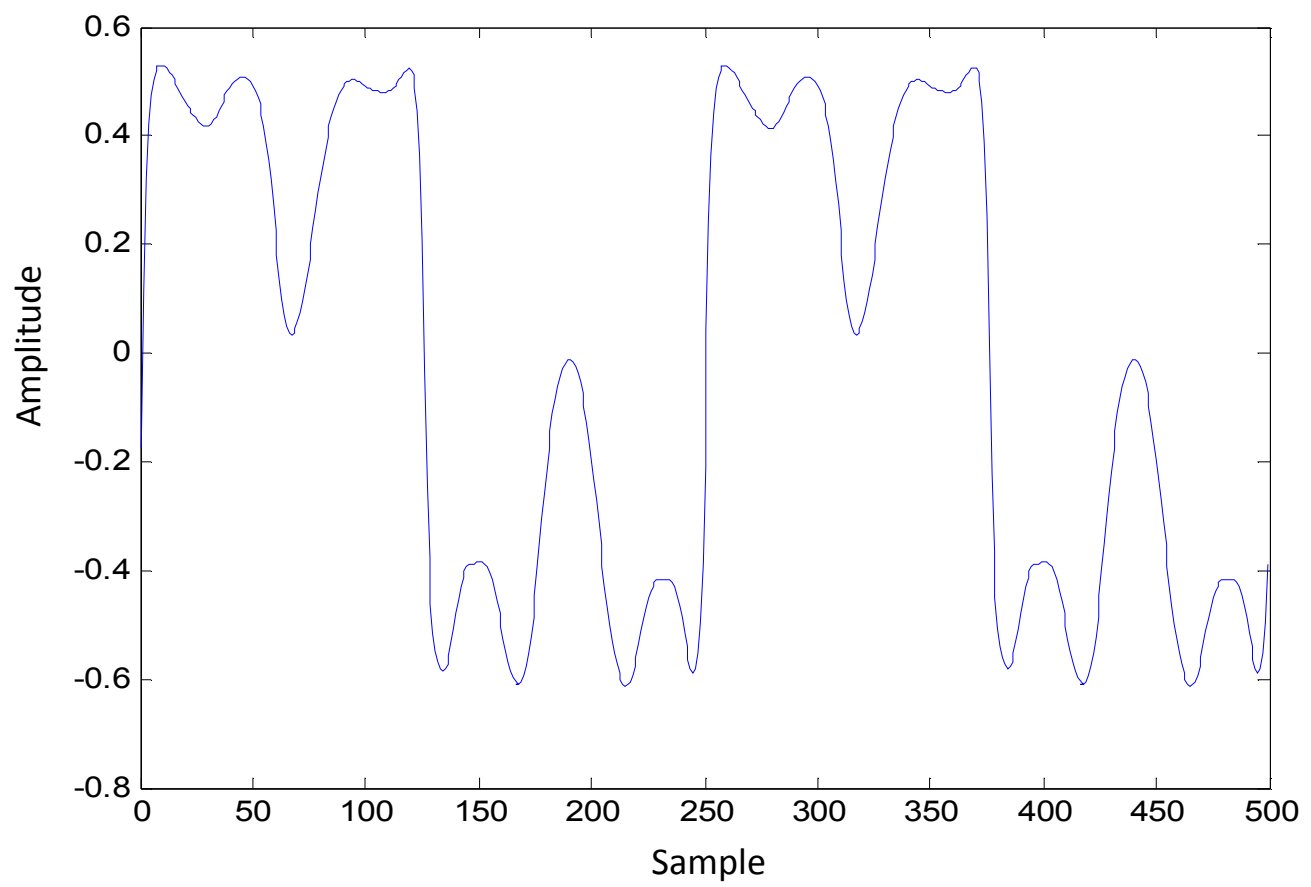

Figure 2.7 Output response of neural network after 200 training iterations of class N(1-86-1) with learning rate $=0.25$ 


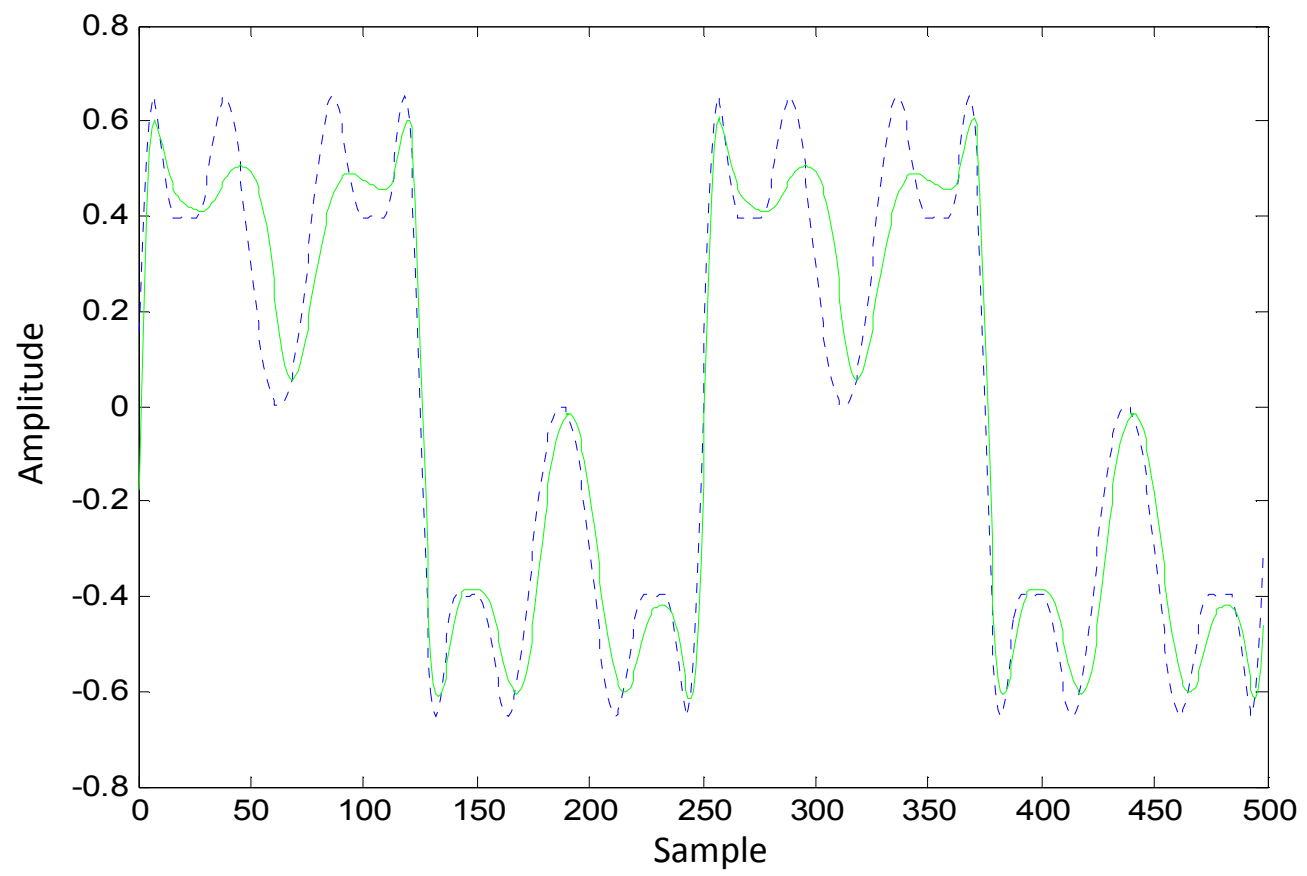

(a) Output comparison between the plant (dashed blue) and neural network (solid green).

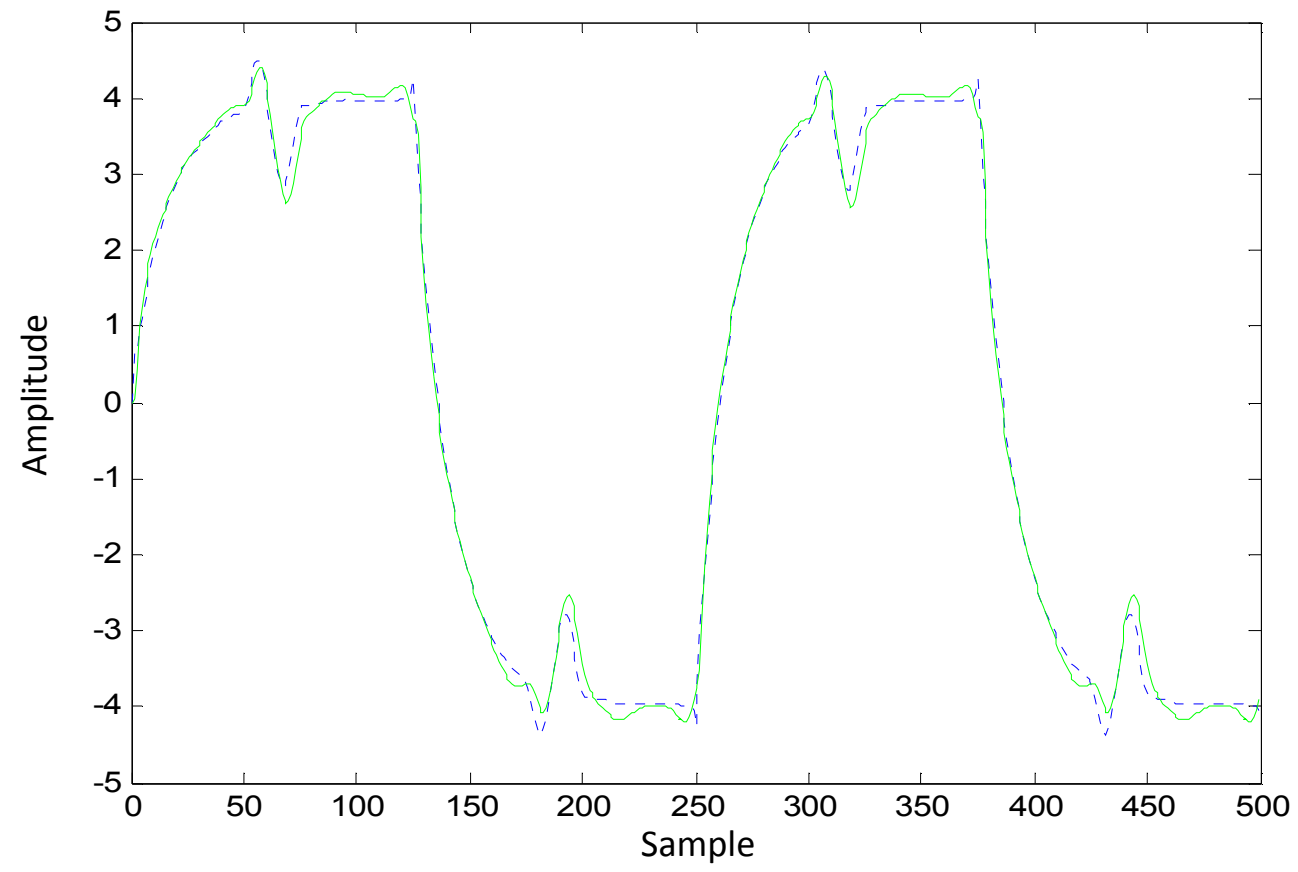

(b) Output comparison from the difference equation governing plant (dashed blue) and neural network (solid green).

Figure 2.8 Output response of neural network after 1,000 training iterations of class N(18-6-1) with learning rate $=0.25$ comparing with the plant 


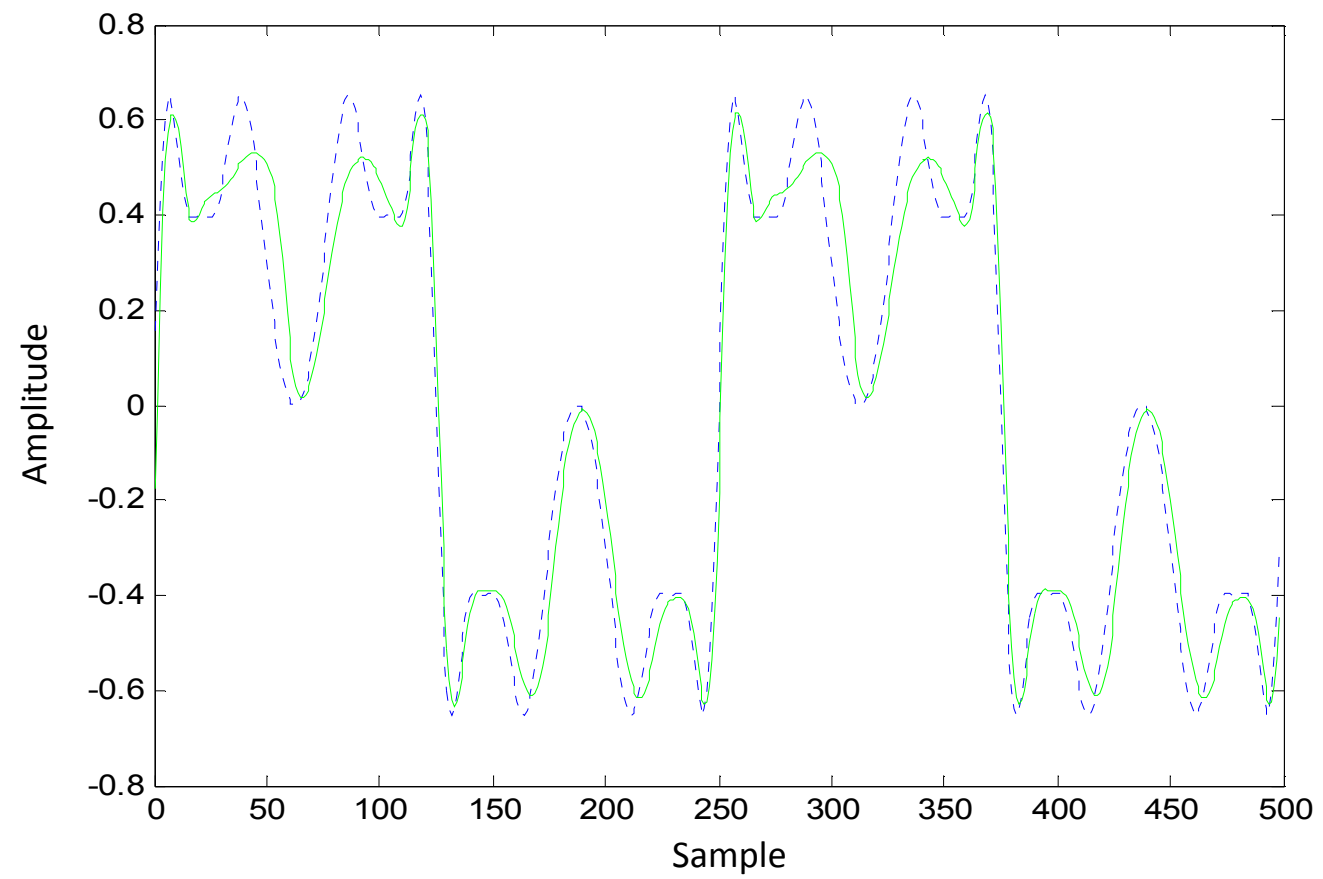

(a) Output comparison between the plant (dashed blue) and neural network (solid green).

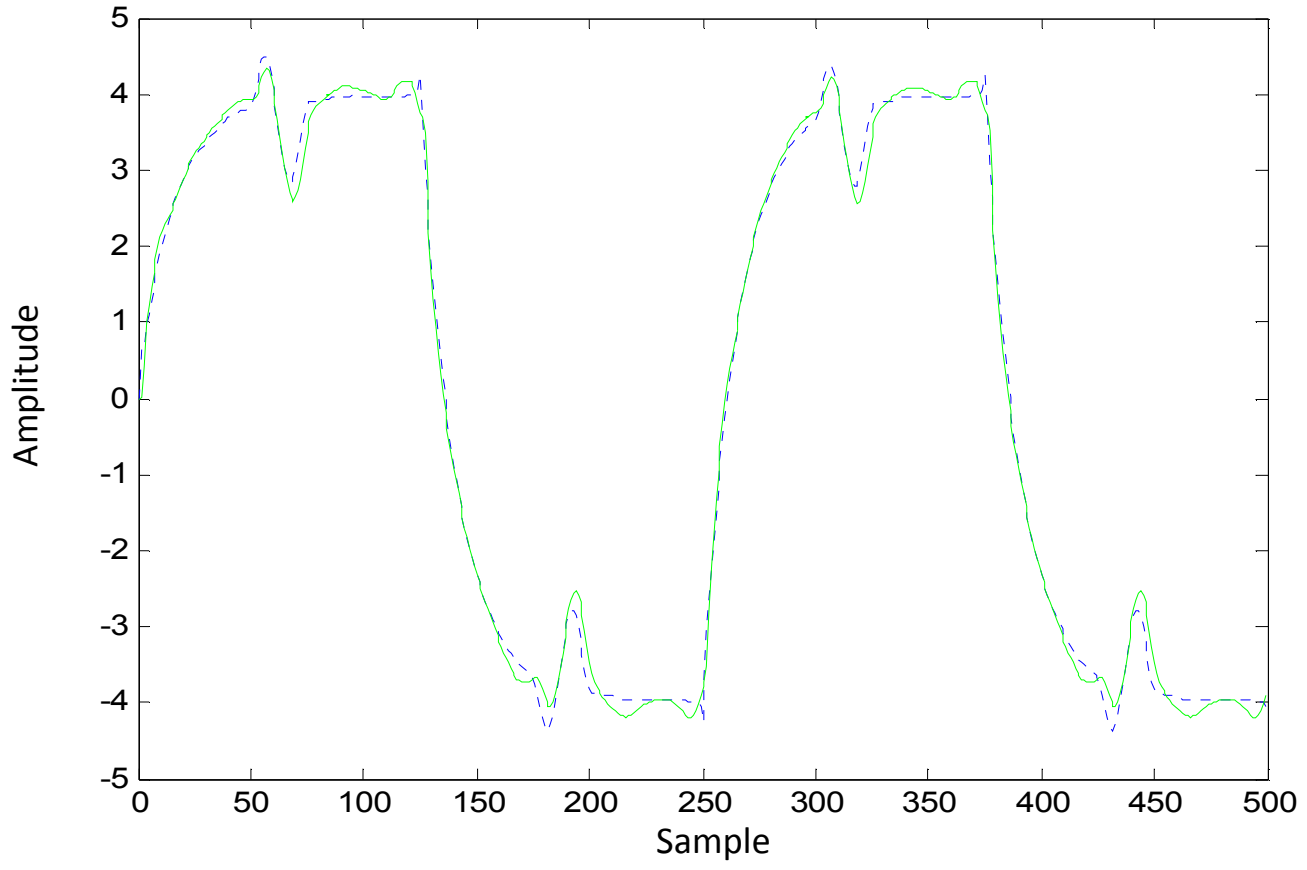

(b) Output comparison from the difference equation governing plant (dashed blue) and neural network (solid green).

Figure 2.9 Output response of neural network after 2,000 training iterations of class N(18-6-1) with learning rate $=0.25$ comparing with the plant 


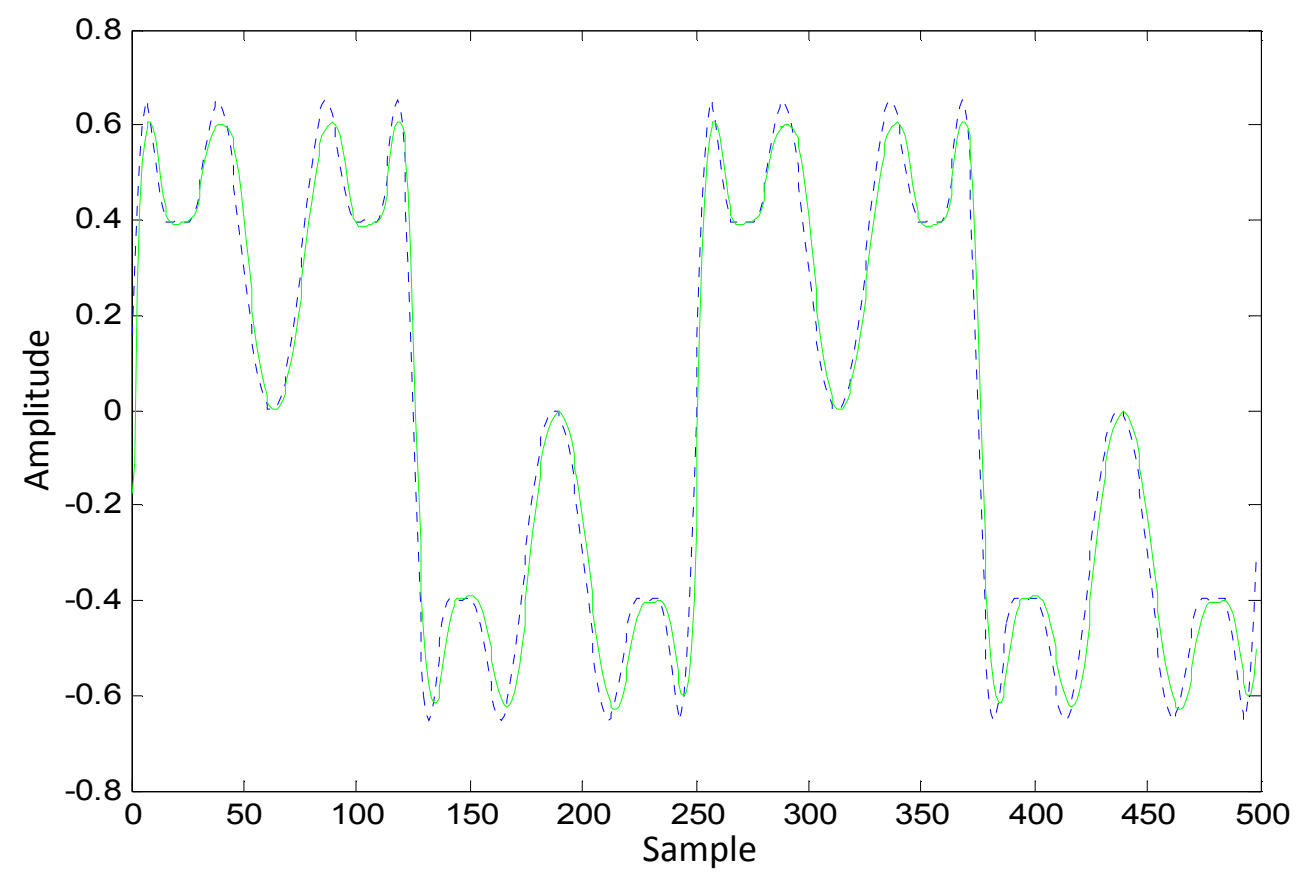

(a) Output comparison between the plant (dashed blue) and neural network (solid green).

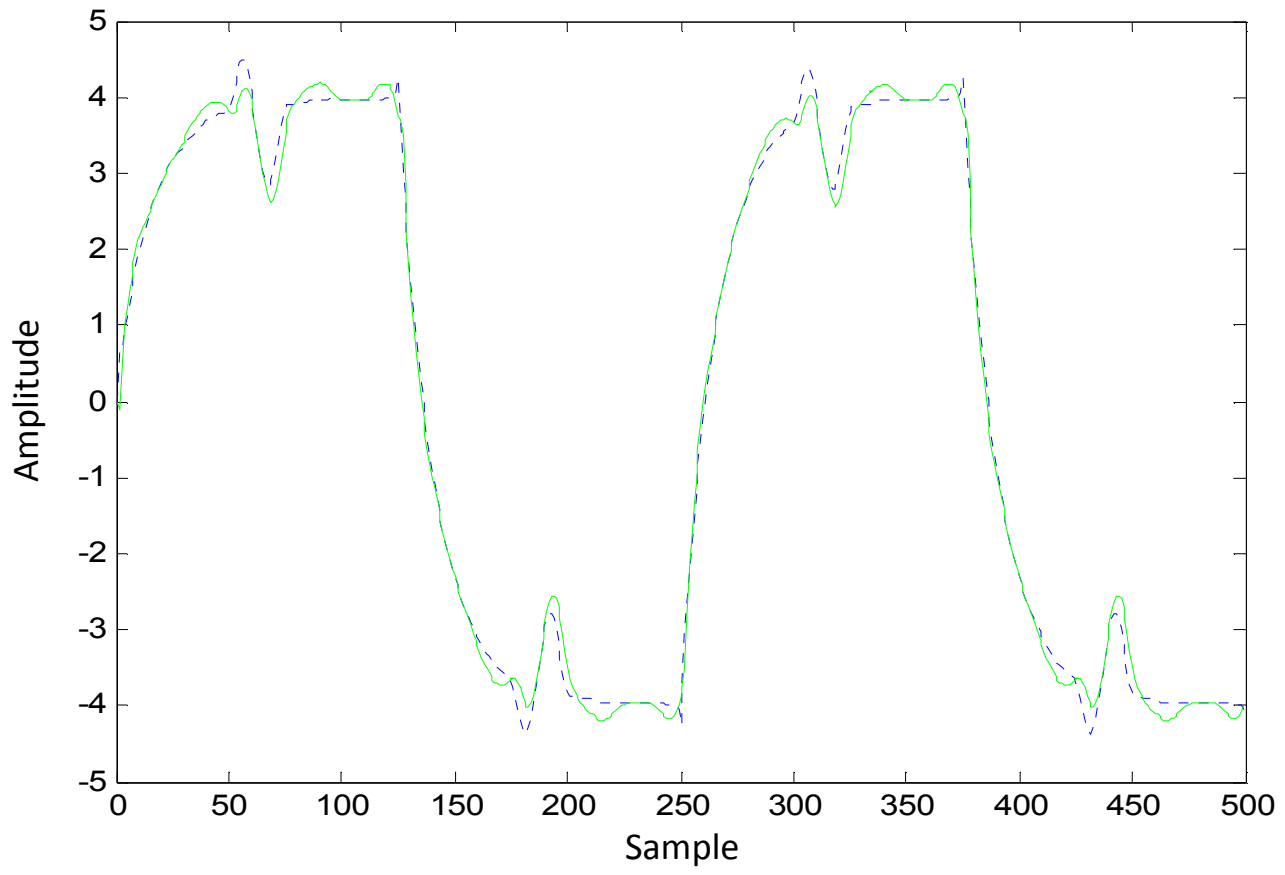

(b) Output comparison from the difference equation governing plant (dashed blue) and neural network (solid green).

Figure 2.10 Output response of neural network after 2,000 training iterations of class $N(1-8-6-1)$ with learning rate $=0.5$ comparing with the plant 


\section{Chapter 3: Introduction to Wavelet}

The wavelet transform is a mathematical concept developed to convert a function or signal into another form which either makes certain features of the original signal more clear to study or be identified. It is well known from Fourier analysis which expresses a signal as the sum of series of sines and consines. The big difference between the Fourier and the wavelet transform is that Fourier expansion has only frequency and no time resolution. The wavelet transform provides a way to represent signal in both time and frequency.

\subsection{Wavelet}

Wavelet, literally, means "small wave." Wavelet is a wave that grows and decays in a finite time interval. For a wave to be classified as a wavelet, all of the following properties must be met [16]:

Let function $\psi(t)$ represents a wave signal:

1. A wavelet must have finite energy:

$$
E=\int_{-\infty}^{\infty}|\psi(t)|^{2} d t<\infty
$$


Note that if $\psi(t)$ is a complex function, the magnitude must be found using both real and complex parts.

2. If $\hat{\psi}(f)$ is the Fourier transform of $\psi(t)$, i.e.

$$
\hat{\psi}(f)=\int_{-\infty}^{\infty} \psi(t) e^{-i(2 \pi f) t} d t
$$

then the following condition must hold:

$$
C_{\psi}=\int_{0}^{\infty} \frac{|\widehat{\psi}(f)|^{2}}{f} d f<\infty
$$

The constant $C_{\psi}$ is known as the admissibility constant and depends on wavelets.

3. Lastly, for complex wavelets, the Fourier transform must both be real and vanish for negative frequencies.

$$
\left.|\widehat{\psi}(f)|^{2}\right|_{f \leq 0}=0
$$

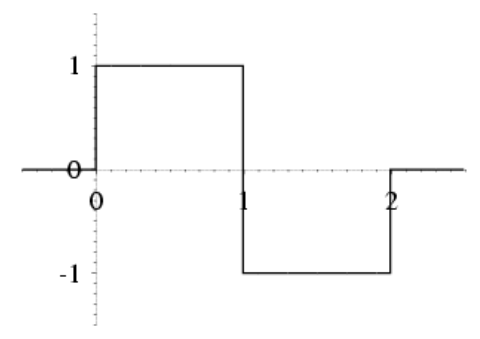

(a)
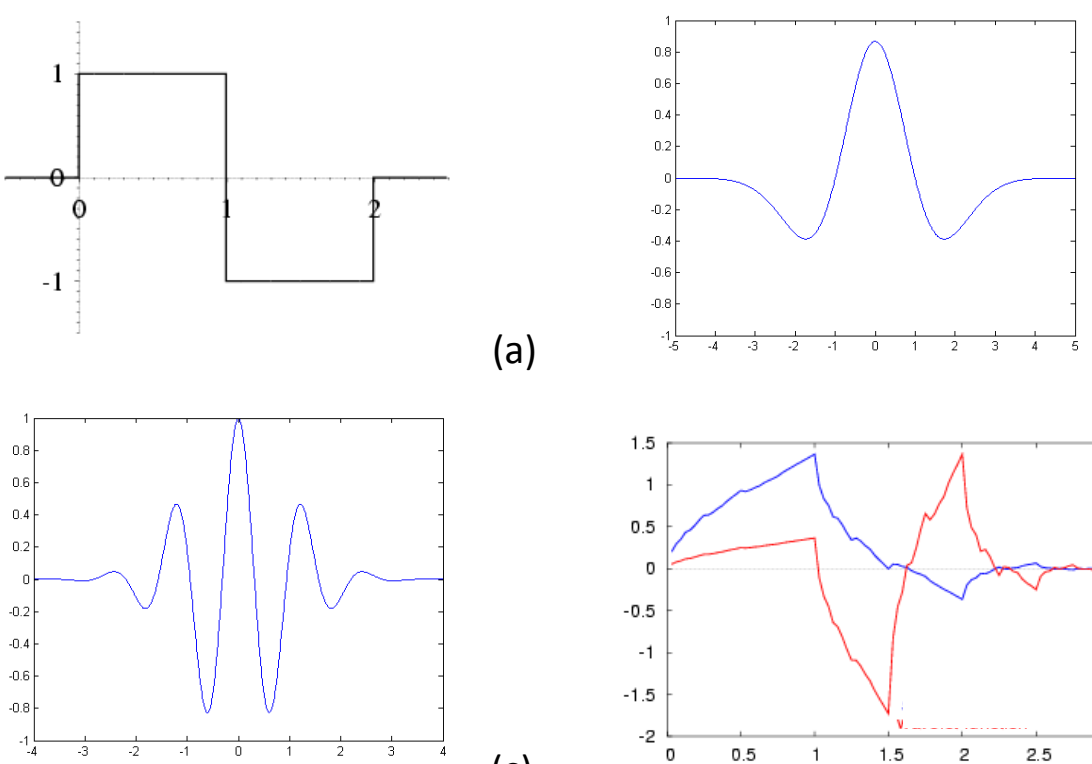

(c)

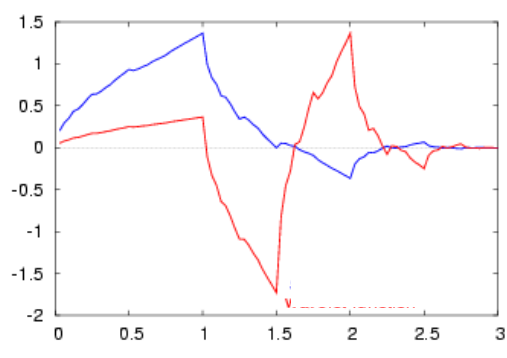

(b)

Figure 3.1 Examples of some of the common wavelet:

(a) Haar wavelet. (b) Mexican hat wavelet. (c) Morlet wavelet. (d) Daubechies wavelet D4 [28]. 


\subsection{Wavelet Transform}

In the operation of a wavelet transform, various wavelets are generated from a single basic wavelet $\psi(t)$, also known as mother wavelet. This is done by introducing the scale factor (or dilation), $s$, and the translation factor (or shift), $\tau$. The shifted and dilated versions of the mother wavelet are denoted by $\psi\left(\frac{t-\tau}{s}\right)$ or simply $\psi_{s, \tau}(t)$.

$$
\psi_{s, \tau}(t)=\frac{1}{\sqrt{s}} \psi\left(\frac{t-\tau}{s}\right)
$$

Utilizing the equation form above, a signal $x(t)$ can be transformed. The wavelet transform of a signal with respect to the wavelet function can be written as:

$$
T(s, \tau)=\frac{1}{\sqrt{s}} \int_{-\infty}^{\infty} x(t) \psi^{*}\left(\frac{t-\tau}{s}\right) d t
$$

The asterisk indicates that the complex conjugate of the wavelet function is used in the transform. The wavelet transform can be thought of as the cross-correlation of a signal with a set of wavelets of various "widths."

\subsection{Inverse Wavelet Transform}

The inverse transformation of wavelet is defined as:

$$
x(t)=\frac{1}{C_{\psi}} \int_{-\infty}^{\infty} \int_{0}^{\infty} T(s, \tau) \psi_{s, \tau}(t) \frac{d s d \tau}{s^{2}}
$$


From the inspection, the wavelet inversion is performed over the integration of both scales, $s$, and location, $\tau$. If the integration of $s$ is to be limited to certain range, say, from some number $s_{0}$ to $\infty$ rather than 0 to $\infty$, a basic low-pass filter can be made. The high frequency components are often noises and can be removed from the reconstructed signal by this method.

\subsection{Discrete Wavelet Transform}

Recall that in a continuous wavelet transform, the process involves correlation between input signal and a wavelet at different scales and translation. In implementing the discrete wavelet transform, a continuous time signal is sampled to form a discrete time signal. This includes the values of scale and translation of wavelet function as well. The discrete form of wavelet hence becomes:

$$
\psi_{m, n}(t)=\frac{1}{\sqrt{s_{0}^{m}}} \psi\left(\frac{t-n s_{0}^{m} \tau_{0}}{s_{0}^{m}}\right)
$$

where the integers $m$ and $n$ control the wavelet scale and translation. Note that the discrete wavelets are not time-discrete, only the translation and scale step are discrete. A parameter $s_{0}$ is a fixed scaling step and must be greater than one while $\tau_{0}$ is the location parameter which must be greater than zero. The common choice for parameters $s_{0}$ and $\tau_{0}$ are: 2 and 1 , respectively. These parameters are also used in the implementation presented at the end of this chapter. Discrete scale and translation 
wavelets are commonly chosen to be orthonormal. These wavelets are both orthogonal to each other and normalized to have unit energy.

$$
\int_{-\infty}^{\infty} \psi_{m, n}(t) \psi_{m^{\prime}, n^{\prime}}(t) d t= \begin{cases}1, & \text { if } m=m^{\prime} \text { and } n=n^{\prime} \\ 0, & \text { otherwise }\end{cases}
$$

This means that the information stored in a wavelet coefficient $T_{m, n}$ is not repeated elsewhere, and allows for the complete regeneration of the original signal without redundancy. Using discrete wavelet representation, the wavelet transform of a signal $x(t)$ can be written as:

$$
T_{m, n}=\frac{1}{\sqrt{s_{0}^{m}}} \int_{-\infty}^{\infty} x(t) \psi\left(\frac{t-n s_{0}^{m} \tau_{0}}{s_{0}^{m}}\right) d t
$$

where $T_{m, n}$ are the discrete wavelet transform values given on a scale-location grid of index $m, n$. In similar fashion, the reconstruction formula is:

$$
x(t)=\sum_{m=-\infty}^{\infty} \sum_{n=-\infty}^{\infty} T_{m, n} \psi_{m, n}(t)
$$

\subsection{Scaling Function}

The third property of wavelet provided that Fourier transform must be both real and vanish at zero and negative frequencies. In a sense, the wavelet has a band-pass like spectrum. From Fourier's theory, one finds that compression in time is equivalent to stretching the spectrum and shifting it upwards: 


$$
F\{x(a t)\}=\frac{1}{|a|} X\left(\frac{2 \pi f}{a}\right)
$$

Hence, if a time compression of the wavelet has a factor of 2, this will also stretch the frequency spectrum of the wavelet by a factor of 2 and shift all frequency components up by a factor of 2 . The wavelet spectrum resulting from scaling of the mother wavelet is shown in figure 3.2 .

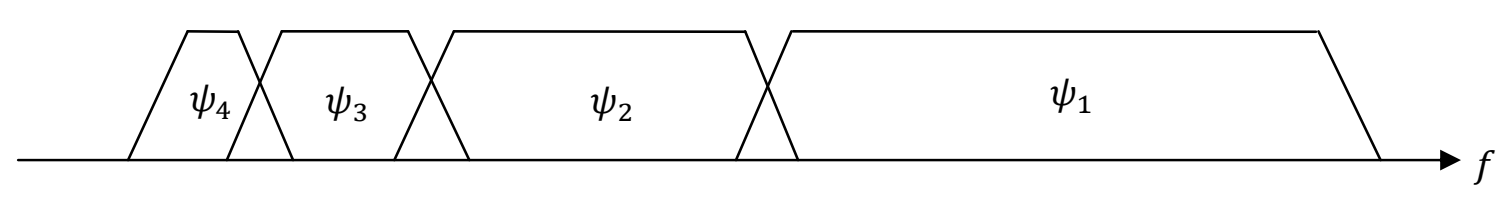

Figure 3.2 Wavelet spectrum resulting from scaling of mother wavelet.

As demonstrated in figure 3.2, wavelet and its scaled version can be thought of as series of band-pass filters. However, due to the fact that the wavelet is stretched in the time domain with a factor of 2 , its bandwidth will always be halved. This is problematic because in order to cover all the spectrums, there needs to be infinite number of wavelets. The easiest way to solve this problem is simply not to cover the spectrum all the way down to zero. See figure 3.3.

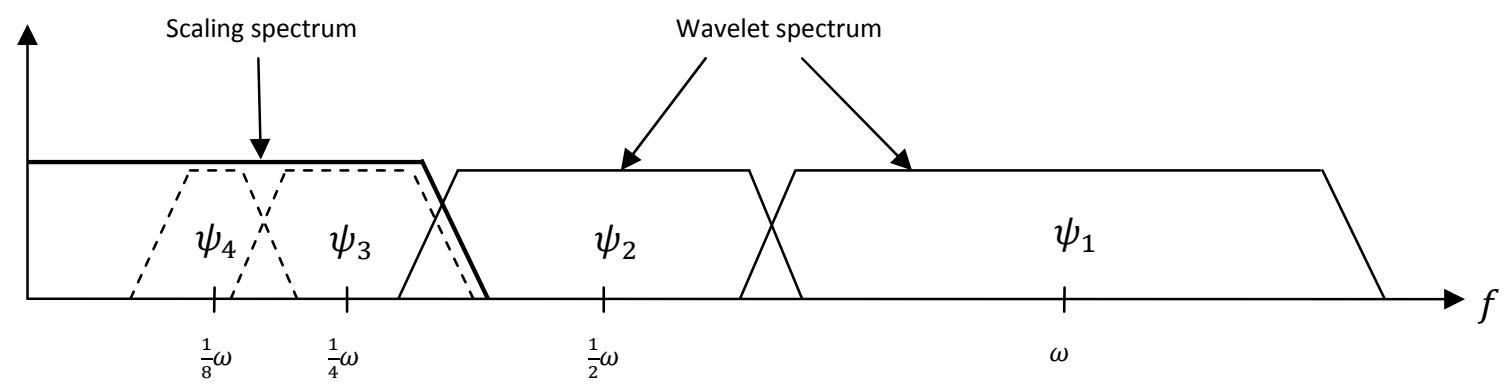

Figure 3.3 Using scaling spectrum to replace infinite set of wavelet spectrum at the lower frequency. 
The remaining spectrum is called scaling spectrum. The advantage of this is that infinite numbers of wavelets are no longer required. On the other hand, looking from the wavelet analysis point of view, information contained within the scaling spectrum will not get transformed. Hence, possible valuable information contained within the scaling spectrum cannot be fully analyzed. Therefore, prior knowledge regarding the frequency scale is important in determining how many decomposing levels are needed for efficient analysis of a given signal.

\subsection{Sub-band Coding and Multi-Resolution}

If the wavelet transform is regarded as a series of filters, then wavelet transform of a signal is simply the signal passing through series of filters. This type of signal analysis is called sub-band coding, much similar to figure 3.3. In wavelet transformation, the signal is usually split into two equal parts: low frequency and high frequency. The high frequency part is referred to as wavelet filter and low frequency part is referred to as a scaling filter. Wavelet filters contain the information of interest. However, the scaling filter contains some additional detail which can be split again. The process of splitting the spectrum is shown in figure 3.4. 


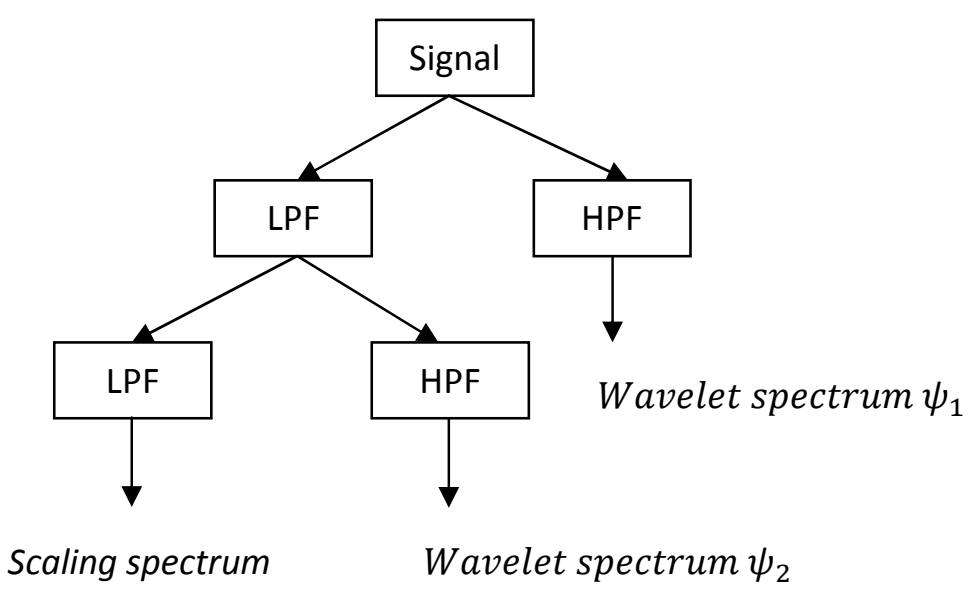

Figure 3.4 Splitting of signal via high-pass filter (HPF) and low-pass filter (LPF) according to figure 3.3.

\subsection{Noise Reduction via Thresholding Method}

In this section, a simple technique of wavelet transform for noise reduction applications will be demonstrated. In performing noise reduction, the method of thresholding is chosen due to its straight forward procedure and simplicity. The thresholding parameter is calculated using the Universal Threshold [11]:

$$
\delta=\sqrt{2 \sigma^{2} \ln (N)}
$$

where $N$ is length of input signal. Variable $\sigma$ is a variance and can be calculated using Median Absolute Deviation or MAD, defined by

$$
\sigma_{M A D}=\operatorname{median}\left(\left|X_{i}-\tilde{X}\right|\right)
$$

where $\tilde{X}$ is the median of the data and $|X|$ is the absolute value of $X$. Unlike other methods, the MAD-deviation can be used to estimate the scale parameter of 
distributions of which the variance and standard deviation do not exist. Even when working with distributions for which the variance exists, the MAD has advantages over standard deviation. For instance, the MAD is more resilient to outliers in a data set. In the standard deviation, the distances from the mean are squared. On average, large deviations are weighted more heavily. In MAD, the magnitude of the distance of a small number of outliers is irrelevant [12]. Following this approach, level-dependent thresholds can be estimated from the wavelet coefficient. At a different level, the MADstandard deviation is calculated as the median absolute deviation divided by

$$
\Phi^{-1}\left(\frac{3}{4}\right) \approx 0.6745
$$

or third quartile of normal distribution. In the thresholding method, the coefficients are compared with a threshold $\delta$. If the coefficient exceeds $\delta$, its value is left unchanged and zero otherwise:

$$
\psi_{i}^{\text {threshold }}=\left\{\begin{aligned}
0, & \text { if }\left|\psi_{i}\right| \leq \delta \\
\psi_{i}, & \text { else }
\end{aligned}\right.
$$

\subsection{Decomposition and Reconstruction}

As mentioned earlier, the step parameters of the wavelets are chosen as following: $s_{0}=2$ and $\tau_{0}=1$. And the wavelet implementation used here is Daubechies wavelets. The coefficients for Daubechies wavelet up to D16 are listed in table 1. The order of Daubechies wavelets relates to its minimum support length of the signal $N-1$. For 
instance, the D2 (Haar) wavelet has a support length of 1, the D4 wavelet has support length of 3, and the D6 a support length of 5 and so on.

Figure 3.5 provides some example of frequency spectrum of different order of Daubechies wavelets. Their characteristic is that of the low-pass filter. As described in earlier section, these are called scaling functions: the higher the order the better the response of the model. However, the higher order will also limit the number of resolution because order of Daubechies wavelets determines the minimum support length of the signal. Hence there is a trade-off between order and resolution. Depending on the application, Daubechies wavelet order should be chosen according to the needs.

Table 3.1 Daubechies Wavelet coefficients D2 to D16 [3]

\begin{tabular}{|r|c|c|c|c|c|c|c|}
\hline $\begin{array}{c}\text { D2 } \\
\text { (Haar) }\end{array}$ & D4 & D6 & D8 & D10 & D12 & D14 & D16 \\
\hline 1 & 0.683013 & 0.470467 & 0.325803 & 0.226419 & 0.157742 & 0.110099 & 0.076956 \\
\hline 1 & 1.183013 & 1.141117 & 1.010946 & 0.853944 & 0.699504 & 0.560791 & 0.442467 \\
\hline & 0.316987 & 0.650365 & 0.892201 & 1.024327 & 1.062264 & 1.031148 & 0.955486 \\
\hline & -0.18301 & -0.19093 & -0.03958 & 0.195767 & 0.445831 & 0.664372 & 0.827817 \\
\hline & & -0.12083 & -0.26451 & -0.34266 & -0.31999 & -0.20351 & -0.02239 \\
\hline & & 0.049818 & 0.043616 & -0.0456 & -0.18352 & -0.31684 & -0.40166 \\
\hline & & & 0.046504 & 0.109703 & 0.137888 & 0.100847 & $6.68 \mathrm{E}-04$ \\
\hline & & & -0.01499 & -0.00883 & 0.038923 & 0.114003 & 0.182076 \\
\hline & & & & -0.01779 & -0.04466 & -0.05378 & -0.02456 \\
\hline & & & & $4.72 \mathrm{E}-03$ & $7.83 \mathrm{E}-04$ & -0.02344 & -0.06235 \\
\hline & & & & & $6.76 \mathrm{E}-03$ & 0.01775 & 0.019772 \\
\hline & & & & & $-1.52 \mathrm{E}-03$ & $6.08 \mathrm{E}-04$ & 0.012369 \\
\hline & & & & & & $-2.55 \mathrm{E}-03$ & $-6.89 \mathrm{E}-03$ \\
\hline & & & & & & $5.00 \mathrm{E}-04$ & $-5.54 \mathrm{E}-04$ \\
\hline & & & & & & & $9.55 \mathrm{E}-04$ \\
\hline & & & & & & & $-1.66 \mathrm{E}-04$ \\
\hline
\end{tabular}




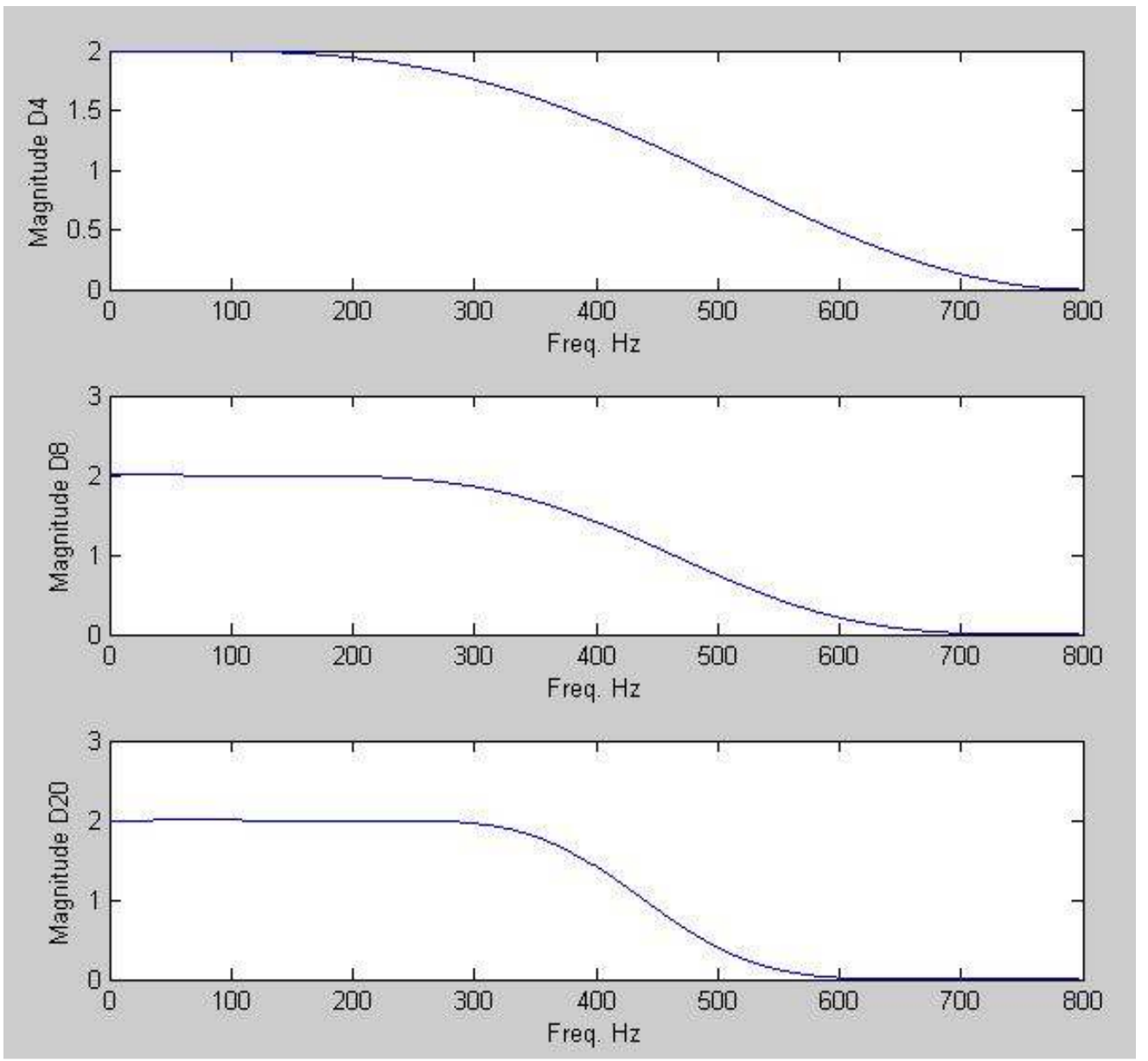

Figure 3.5 Example of Daubechies's frequency spectrum of D4, D8 and D20.

In figure 3.4, transformation structure provides that the signal needs to go through series of high-pass and low-pass filter to obtain scaling and wavelet coefficients of the signal. Low-pass filter can be obtained directly from Daubechies wavelets coefficient. As for the high-pass filter, the coefficient is multiply by $(-1)^{k}$ to acquire the response of high-pass filter. In other words, the sign is simply changed for every odd or even of the Daubechies wavelets coefficient. In this case, the scaling coefficients are computed using: 


$$
S_{m+1, n}=\frac{1}{\sqrt{2}} \sum_{k=0}^{N_{k}-1} c_{k} S_{m, 2 n+k}
$$

Similarly for wavelet coefficient

$$
W_{m+1, n}=\frac{1}{\sqrt{2}} \sum_{k=0}^{N_{k}-1} b_{k} S_{m, 2 n+k}
$$

where $b_{k}=(-1)^{k} c_{N_{k}-1-k}$. The sub-scripts and integers are defined as the following:

$m-$ resolution level

$n$ - coefficient index

$k$ - signal index

$N_{k}$ - total length of signal which vary from level to level

As for the reconstruction, the procedure is simply reverse by tracing the process in figure 3.4 from bottom to top:

$$
S_{m-1, n}=\frac{1}{\sqrt{2}} \sum_{k} c_{n-2 k} S_{m, k}+\frac{1}{\sqrt{2}} \sum_{k} b_{n-2 k} W_{m, k}
$$

Note that $S_{0}$ is simply the original input signal. Figure 3.6 provides a schematic for forward DWT. The sub-sample symbol $\$ 2$ means take every second value of the filtered signal. In continuous wavelet transform, the process of sub-sampling translates to scaling the wavelet with factor of $2,\left(s_{0}=2\right)$ as described in prior section. To produce wavelet coefficients, the filtered signals are convolved with the high-pass filter $c($.$) then$ sub-sampled by 2 . Similarly, scaling coefficients are convolved with low-pass filter $b($. then sub-sampled in the same way as wavelet coefficients. When complete, the wavelet 
coefficients are kept and the scaling coefficients are again passed through the low-pass and high-pass filters that give the components of the next transformation level. The process is repeated over all the scales to give the full decomposition of the signal.

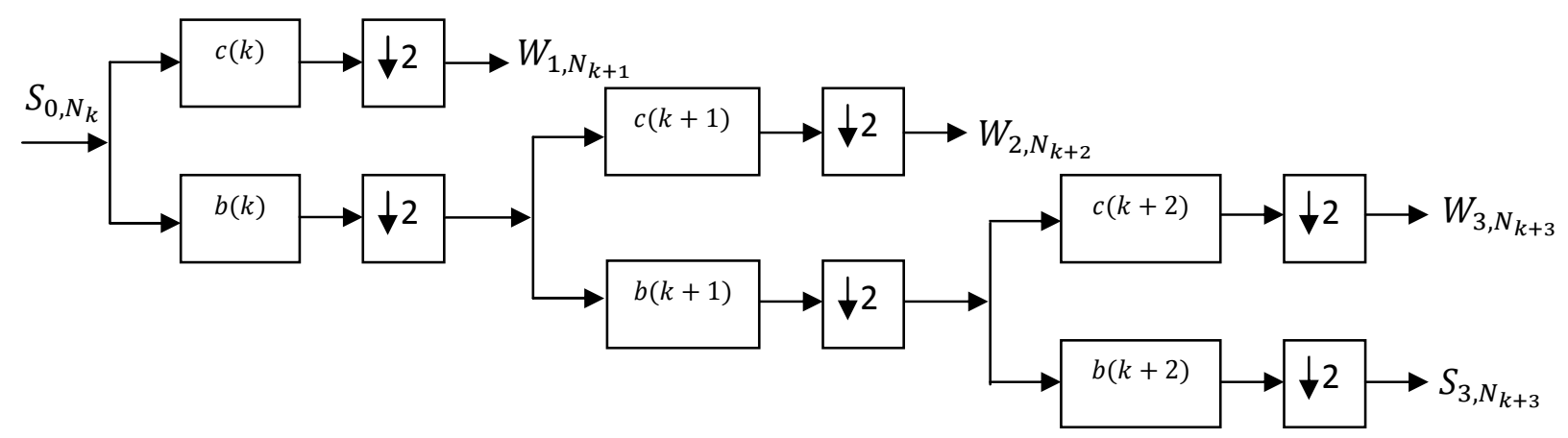

Figure 3.6 Schematic of signal filtering of the forward transform to produce wavelet and scaling coefficient with resolution of 3 levels.

To reverse the process, the wavelet and scaling coefficients at the deepest level are first up-sampled, represents by symbol $\uparrow_{2}$. The up-sample process means that zeros are inserted between the filtered signals. Like the forward transform, the wavelet and scaling coefficients are processed through the high-pass filter and low-pass filter, respectively. This time, however, the filter coefficients are reversed in order. By doing so, the process maintain the condition of perfect reconstruction of the original sequence, undoing any condition caused by the forward process. In a sense, forward and reverse transform are identical to each other except for time reversal. This process repeats until the original length of the signal is recovered. The schematic diagram is shown in figure 3.7 below. 


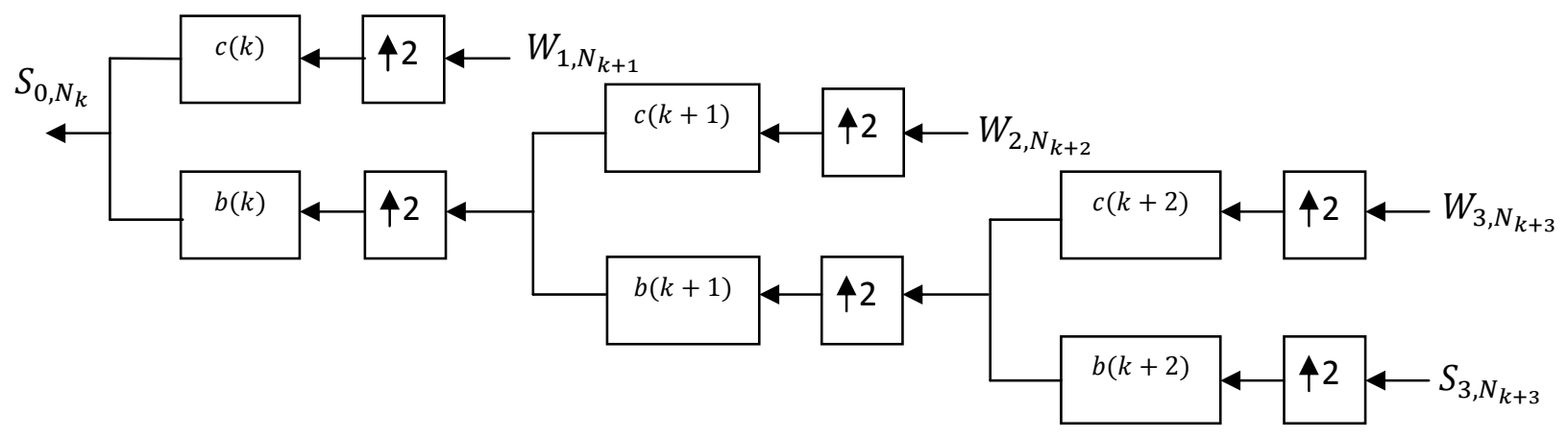

Figure 3.7 Schematic diagram of the filtering of signal reconstruction from wavelet and scaling coefficients with resolution of 3 levels.

\subsection{Implementation}

Wavelet transformation can be used for noise reduction. In the following simulation, it is applied to:

$$
X(t)=\sin (t)+\text { noise } \quad \text { where }\left\{\begin{array}{l}
t=0: 0.01: 10.23 \\
\text { noise }=0.2 \text { randn }(1024)
\end{array}\right.
$$

Daubechies D16 wavelet with resolution of 3 decomposition levels is chosen. The result from the implementation is shown in figure 3.8 and 3.9. In figure 3.8, the upper plot is the original input with $20 \%$ random noise added to the signal. After the first level transformation, wavelet coefficients are plotted in figure (3.8b). Second level coefficients are in figure (3.8c), and third are plotted in figure (3.8d). The scaling coefficients are shown in figure (3.8f). Finally, the reconstruction of the signal is shown in figure (3.8e). Note that noise reduction is not implemented. The result here is to simply to provide analysis of each frequency component level. 
The lower level component of the transformation contains a higher frequency spectrum than the rest. As additional levels are added, the lower frequency spectrum is cut down by half leaving the wavelet coefficients behind and the scaling coefficients to be repeated with the same process. As a result, the lowest frequency component will always be found at the scaling spectrum.

The result from the observation found in figure 3.8 provides vital information to perform the thresholding method. The separation between noise and the target frequency is sufficient with three level transformations. Here, noise reduction via thresholding is applied at every resolution level. The result is shown in figure 3.9. After the thresholding, most of the noise has now been removed and the signal has a much smoother curve. Note that in figure 3.8, for every level, the signal's length is being cut in half. This resulted from the down-sampling process. For reconstruction, the up-sampling lengthens the signal by multiple of two.

Complete software listing can be found in the appendix.

\subsection{Conclusion}

Wavelet transform is a well known method of decomposing signals. In this chapter, its basic concept is introduced. Its application of noise reduction via universal thresholding is also demonstrated. The key idea of this algorithm consists of signal decomposition into multi-band of frequencies. The calculated threshold constants will be applied for 
each level. If the compared signal is equal or below that of the threshold, that signal will be removed (by setting to zero). The result from the implementation provides a satisfactory efficiency of wavelet in signal de-noising application.

The aim of this chapter is to provide a ground work for chapters that follow which involves the concept from previous chapter: neural network, and wavelet. During the past decade, application of both neural networks and wavelets in the fields of mathematics and engineering has grown rapidly. One interesting application is to combine both of these concepts by deploying wavelet as the activation functions in neural network. In the next chapter, this concept will be talked about in more detail. 


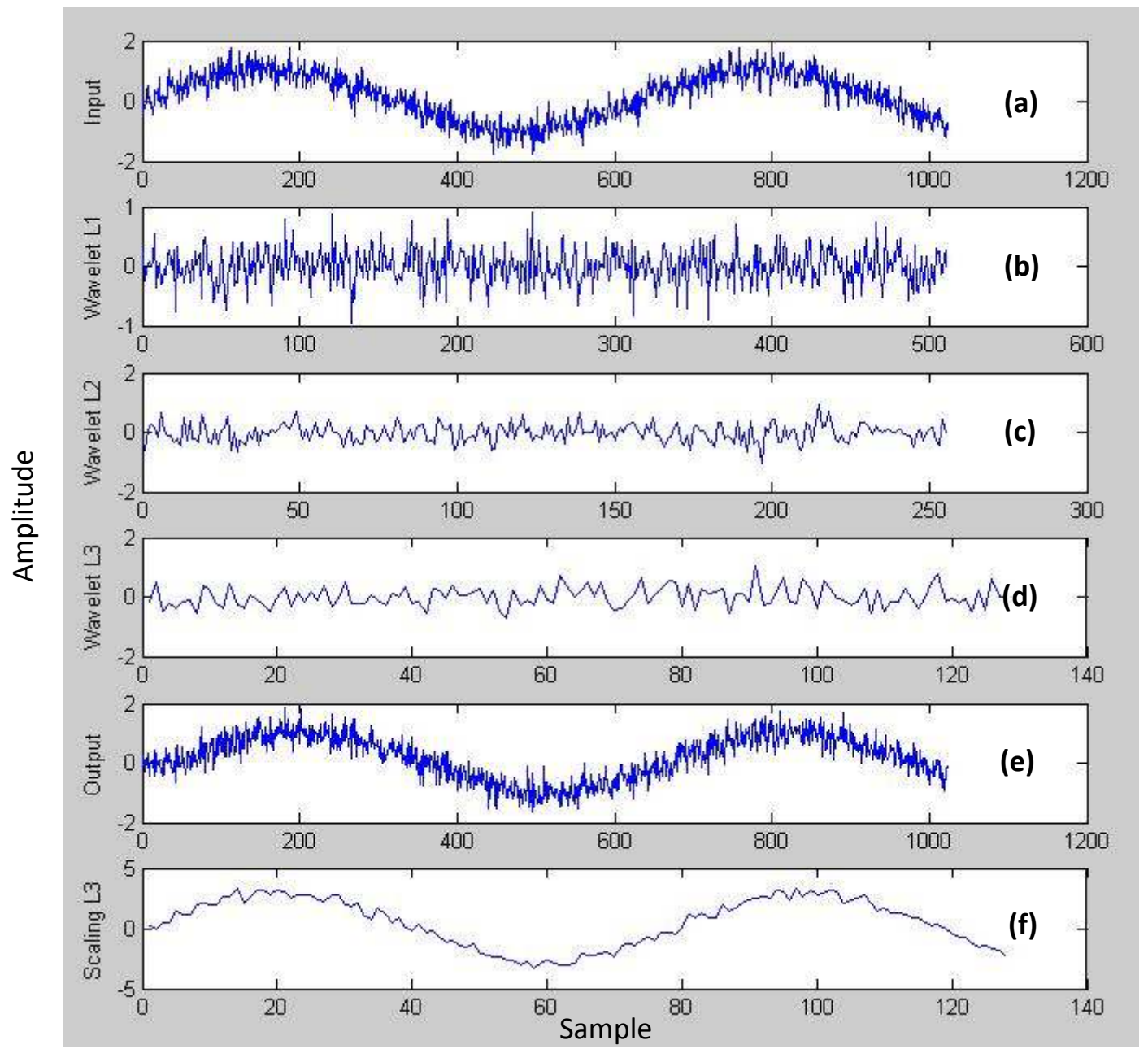

Figure 3.8 DWT using Daubechies wavelet with three levels transformation and 1024 sampling points.

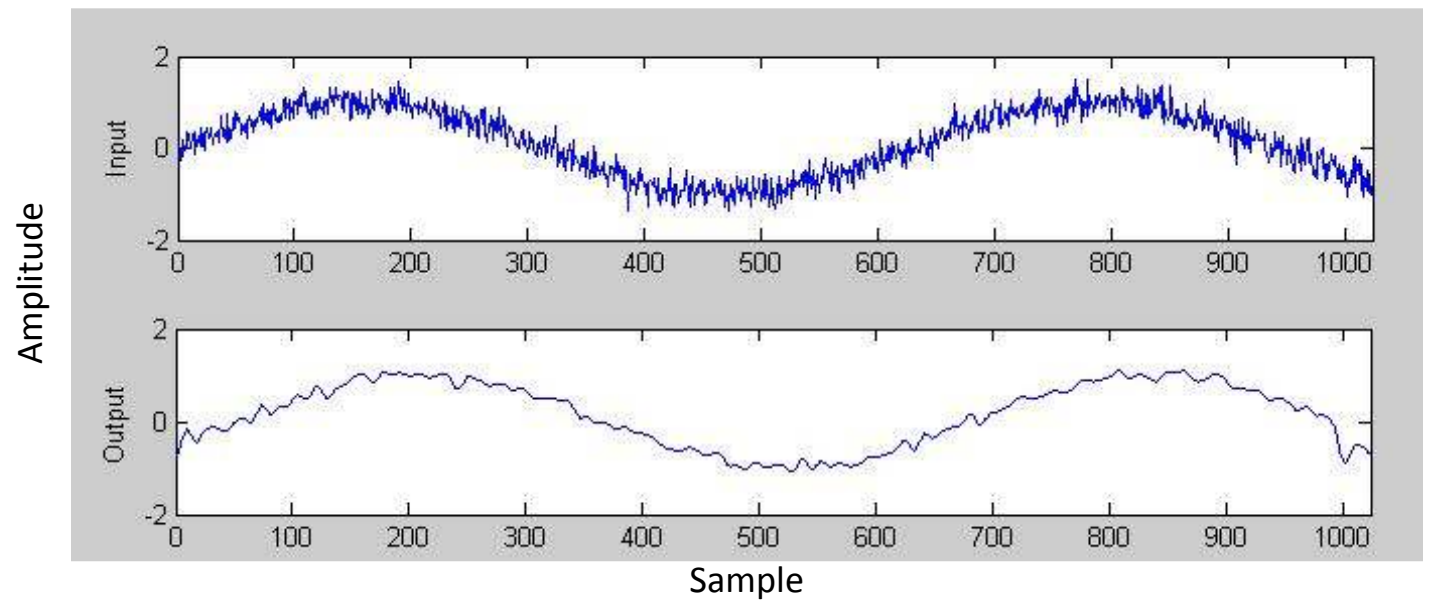

Figure 3.9 The upper plot is the input signal with added-noise. The lower plot is the thresholding signal estimation using Daubechies D16 wavelet transform with three level resolutions. 


\section{Chapter 4: Wavelet Neural Network}

The Wavelet Neural Network (WNN) belongs to a new class of neural networks with unique capabilities in a nonlinear time series analysis. Wavelets are a class of basis functions with oscillations of effectively finite-duration that makes them look like "little waves." The multiple resolution nature of wavelets offer a natural framework for the analysis of physical signals. On the other hand, artificial neural networks constitute a powerful class of nonlinear function approximates for model-free estimation.

The concept of Wavelet Neural Network was inspired by both the technologies of wavelet decomposition and neural networks. In standard neural networks, the nonlinearity is approximated by superposition of sigmoid functions. While in WNN, the nonlinearities are approximated by superposition of a series of wavelet functions. Due to this similarity between the two, combining Wavelet and Neural Network are an attractive idea. 


\subsection{Architectures of WNN}

Basic WNN inherits most of it structural design from Neural Networks that consist of a feed-forward neural network with one hidden layer. An ordinary neuron model (shown in figure 4.1) is characterized by weighted sum of input and an activation function $\phi$. The sum of the weighted-inputs, through the activation function, provides an output of a neuron. In the WNN, the activation functions are drawn from an orthonormal wavelet family $\psi$ which is characterized by scale $s$ and translation $\tau$ (see figure $4.2(\mathrm{~b})$ ). The wavelet neuron is also known as wavelon. Hence, the output of a simple single-inputsingle-output WNN in figure 4.2(a) can be described by the following equation:

$$
g(x)=\sum_{i=1}^{N}\left(w_{i} \Psi_{\mathrm{i}}(x)\right)+\bar{g}
$$

where the additional (and redundant) parameter $\bar{g}$ or bias is introduced to help dealing with nonzero mean functions on finite domains. Note that the output node can either be modeled as another wavelet neuron or simply just a summation. For the purpose of this paper, the summation method will be chosen. The training parameters include the weights $w$, scales $s$, and translations $\tau$ variables. Thus, in addition to connection weights, the optimization of both scales and translations are also needed. The structure of the network for the n-dimensional input space is shown in figure 4.3. In this case, the multivariate wavelet basis function can be calculated by the tensor product of $n$ single wavelet basis function as follows: 


$$
\Psi(\tilde{x})=\prod_{i=1}^{n} \psi\left(x_{i}\right)
$$

Hence, in standard form of WNN, the output is given by

$$
g(\tilde{x})=\sum_{i=1}^{N}\left(w_{i} \Psi_{\mathrm{i}}(\tilde{x})\right)+\bar{g}=\sum_{i=1}^{N}\left(w_{i}\left|\tilde{s}_{i}\right|^{-\frac{1}{2}} \psi\left(\frac{\tilde{x}-\tilde{\tau}_{i}}{\tilde{s}_{i}}\right)\right)+\bar{g}
$$

where:

$$
\begin{aligned}
& \tilde{x}=\left(x_{1}, x_{2}, \ldots, x_{n}\right) \\
& \tilde{\tau}_{i}=\left(\tau_{i, 1}, \tau_{i, 2}, \ldots \tau_{i, n}\right) \\
& \tilde{s}_{i}=\left(s_{i, 1}, s_{i, 2}, \ldots s_{i, n}\right)
\end{aligned}
$$

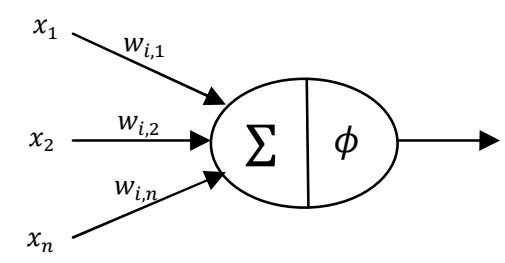

Figure 4.1 A conventional neuron model with activation function

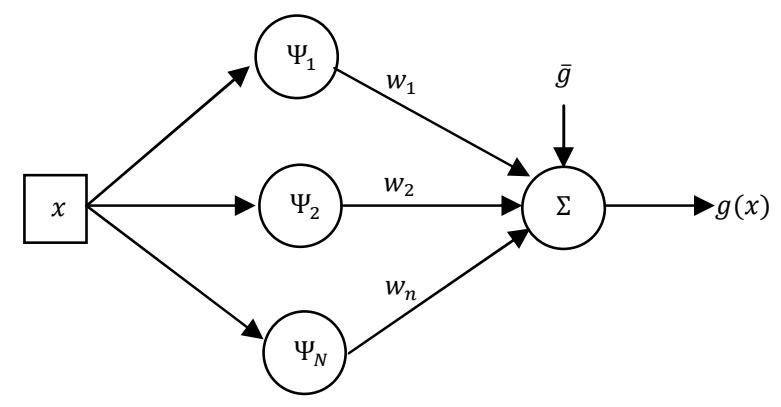

(a)

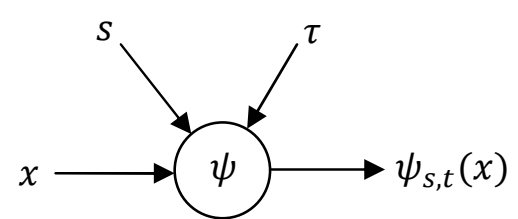

(b)

Figure 4.2 (a) ANN using wavelets as the activation function. (b) Single neuron modeling wavelet. 


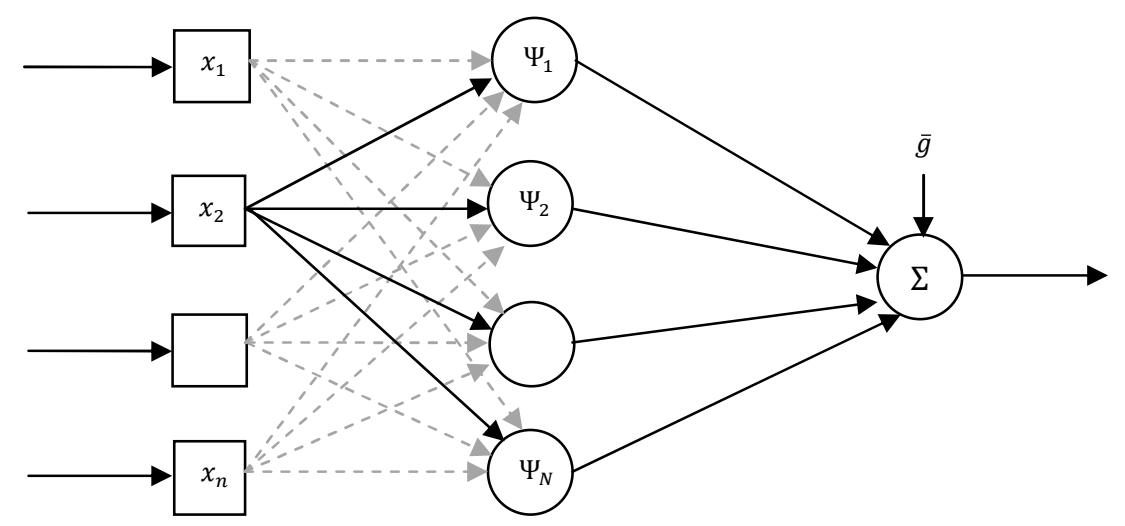

Figure 4.3 Basic wavelet neural network structure.

\subsection{Learning Algorithm}

Denote $\theta$ as the vector collecting all the parameters of the network in equation (4.3) and denote the output as $g_{\theta}(\tilde{x})$. The learning algorithm should minimize the following criterion:

$$
c(\tilde{\theta})=\frac{1}{2} E\left\{\left[g_{\theta}(\tilde{x})-y\right]^{2}\right\}
$$

A gradient algorithm is implemented to recursively minimize the criterion (4.4) using input-output observations. This algorithm modifies the vector $\tilde{\theta}$ after each measurement $\left(\tilde{x}_{k}, y_{k}\right)$ in the opposite direction of the gradient of [3]:

$$
c\left(\tilde{\theta}, \tilde{x}_{k}, y_{k}\right)=\frac{1}{2}\left[g_{\theta}\left(\tilde{x}_{k}-y_{k}\right)\right]^{2}
$$




\subsubsection{Calculating Gradient}

Gradient of the equation (4.5) is taken with regard to each component of the parameter vector $\theta$. For convenience, the following notations will be used:

$$
\begin{gathered}
\psi^{\prime}(\tilde{x})=\frac{d}{d \tilde{x}} \psi(\tilde{x}) \\
e_{k}=g_{\theta}(\tilde{x})-y_{k} \\
\tilde{z}_{i}=\frac{\tilde{x}_{k}-\tilde{\tau}_{i}}{\tilde{s}_{i}}
\end{gathered}
$$

The partial derivatives of the function $c\left(\tilde{\theta}, \tilde{x}_{k}, y_{k}\right)$ with respect to $\bar{g}, w_{i}, \tilde{\tau}_{i}$, and $\tilde{s}_{i}$ are calculated as follow:

$$
\begin{aligned}
\frac{d c}{d \bar{g}} & =\frac{d}{d \bar{g}}\left(\frac{1}{2}\left[g_{\theta}\left(\tilde{x}_{k}\right)-y_{k}\right]^{2}\right) \\
& =g_{\theta}\left(\tilde{x}_{k}\right)-y_{k} \\
& =e_{k} \\
\frac{d c}{d w_{i}} & =\frac{d}{d w_{i}}\left(\frac{1}{2}\left[g_{\theta}\left(\tilde{x}_{k}\right)-y_{k}\right]^{2}\right) \\
& =\left(g_{\theta}\left(\tilde{x}_{k}\right)-y_{k}\right) g_{\theta}^{\prime}\left(\tilde{x}_{k}\right) \\
& =\left(g_{\theta}\left(\tilde{x}_{k}\right)-y_{k}\right) \frac{d}{d w_{i}}\left(\sum_{i=1}^{N} w_{i} \Psi\left(\tilde{z}_{i}\right)+\bar{g}\right) \\
& =\left(g_{\theta}\left(\tilde{x}_{k}\right)-y_{k}\right) \Psi^{\prime}\left(\tilde{z}_{i}\right) \\
& =e_{k} \Psi^{\prime}\left(\tilde{z}_{i}\right) o r e_{k} \psi^{\prime}\left(\tilde{z}_{i}\right) \\
\frac{d c}{d \tilde{\tau}_{i}} & =\frac{d}{d \tilde{\tau}_{i}}\left(\frac{1}{2}\left[g_{\theta}\left(\tilde{x}_{k}\right)-y_{k}\right]^{2}\right) \\
& =\left(g_{\theta}\left(\tilde{x}_{k}\right)-y_{k}\right) g_{\theta}^{\prime}\left(\tilde{x}_{k}\right) \\
& =\left(g_{\theta}\left(\tilde{x}_{k}\right)-y_{k}\right) \frac{d}{d \tilde{\tau}_{i}}\left(\sum_{i=1}^{N} w_{i} \Psi\left(\tilde{z}_{i}\right)+\bar{g}\right) \\
& =\left(g_{\theta}\left(\tilde{x}_{k}\right)-y_{k}\right) w_{i} \Psi^{\prime}\left(\tilde{z}_{i}\right) \\
& =\left(g_{\theta}\left(\tilde{x}_{k}\right)-y_{k}\right) w_{i} \frac{d}{d \tilde{\tau}_{i}}\left(\left|\tilde{s}_{i}\right|^{-\frac{1}{2}} \psi\left(\frac{\tilde{x}-\tilde{\tau}_{i}}{\tilde{s}_{i}}\right)\right)
\end{aligned}
$$




$$
\begin{aligned}
& =\left(g_{\theta}\left(\tilde{x}_{k}\right)-y_{k}\right) w_{i}(-1)\left(\frac{1}{\tilde{s}_{i}}\right) \psi^{\prime}\left(\tilde{z}_{i}\right) \\
& =-e_{k} w_{i}\left(\frac{1}{\tilde{s}_{i}}\right) \psi^{\prime}\left(\tilde{z}_{i}\right) \\
\frac{d c}{d \tilde{s}_{i}} & =\frac{d}{d \tilde{s}_{i}}\left(\frac{1}{2}\left[g_{\theta}\left(\tilde{x}_{k}\right)-y_{k}\right]^{2}\right) \\
& =\left(g_{\theta}\left(\tilde{x}_{k}\right)-y_{k}\right) g_{\theta}^{\prime}\left(\tilde{x}_{k}\right) \\
& =\left(g_{\theta}\left(\tilde{x}_{k}\right)-y_{k}\right) \frac{d}{d \tilde{s}_{i}}\left(\sum_{i=1}^{N} w_{i} \Psi\left(\tilde{z}_{i}\right)+\bar{g}\right) \\
& =\left(g_{\theta}\left(\tilde{x}_{k}\right)-y_{k}\right) w_{i} \Psi^{\prime}\left(\tilde{z}_{i}\right) \\
& =\left(g_{\theta}\left(\tilde{x}_{k}\right)-y_{k}\right) w_{i} \frac{d}{d \tilde{s}_{i}}\left(\left|\tilde{s}_{i}\right|^{-\frac{1}{2}} \psi\left(\frac{\tilde{x}-\tilde{\tau}_{i}}{\tilde{s}_{i}}\right)\right) \\
& =\left(g_{\theta}\left(\tilde{x}_{k}\right)-y_{k}\right) w_{i}(-1)\left(\frac{1}{\tilde{s}_{i}^{2}}\right)\left(\tilde{x}_{k}-\tilde{\tau}_{i}\right) \psi^{\prime}\left(\tilde{z}_{i}\right) \\
& =-e_{k} w_{i}\left(\frac{1}{\tilde{s}_{i}^{2}}\right)\left(\tilde{x}_{k}-\tilde{\tau}_{i}\right) \psi^{\prime}\left(\tilde{z}_{i}\right)
\end{aligned}
$$

\subsubsection{Parameters Initialization}

Wavelet Neural Network parameters initialization is done according to [3]. For the sake of simplicity, the one dimensional case will be considered. Assume $f(x)$ is a function to be approximated over the range $\mathcal{D}=[a, b]$. The output of wavelet neural network can be written as:

$$
g(x)=\sum_{i=1}^{N}\left(w_{i} \psi\left(\frac{x-\tau_{i}}{s_{i}}\right)\right)+\bar{g}
$$

The initialization of this network includes the initialization of parameters $\bar{g}, w_{i}, \tau_{i}$, and $s_{i}$ for $i=1,2 \ldots N$. The estimation of the mean of function $f(x)$ is used to initialize $\bar{g}$. Variables $w_{i}$ 's are set to a random number or just simply set to zero. A method to initialize $\tau_{i}{ }^{\prime}$ s, and $s_{i}{ }^{\prime}$ s also needs to be determined. 
To initialize $t_{1}$ and $s_{1}$, select a point $p$ between $a$ and $b: a<p<b$.

$$
p=\int_{a}^{b} x \rho(x) d x
$$

and

$$
\rho(x)=\frac{\varrho(x)}{\int_{a}^{b} \varrho(x) d x}, \text { where } \varrho(x)=\left|\frac{d f(x)}{d x}\right|
$$

which must be estimated from noisy input/output observations $\{x, f(x)\}$. The calculation $t_{1}$ and $s_{1}$, then

$$
t_{1}=p, s_{1}=\xi(b-a)
$$

A constant $\xi$ is usually chosen to be 0.5 . Now the interval $[a, b]$ is divided into two subintervals. The process keeps repeating itself recursively on each sub-interval. As for the multidimensional case, $\bar{g}$, and $w_{i}$ 's are handled the same way. To initialize $\tilde{t}_{1}$ and $\tilde{s}_{1}$, the procedures are handled separately for different coordinates.

\subsection{Implementation}

A Wavelet Neural Network model is implemented to simulate function approximation.

A wavelet function chosen for this experiment is Gaussian-derivative wavelet family:

$$
\psi(x)=-x e^{-\frac{1}{2} x^{2}}
$$

The target function to be approximate is defined by piecewise function:

$$
f(x)=\left\{\begin{array}{lr}
-2.186 x-12.864, & -10 \leq x<-2 \\
4.246 x, & -2 \leq x<0 \\
10 e^{-0.05 x-0.5} \sin ((0.03+0.7) x), & 0 \leq x \leq 10
\end{array}\right.
$$


over the domain $\mathcal{D}=[-10,10]$ uniformly sampled set of 201 points. Class $W_{N N_{1,7,1}}$ is trained for 10,000 iterations. The result is shown in figure 4.4 . The original function is displayed in green while the approximated WNN is shown in blue. In this case, the experimental learning rate is 0.02 . A piecewise function equation (4.12) is widely studied by many researchers in the field of neural networks [3][35][36]. This function is continuous and analyzable. However, traditional analytical tools become inefficient and often fail due to two reasons, namely, the wide-band information hidden at the turning points and the coexistence of linearity and nonlinearity [36].

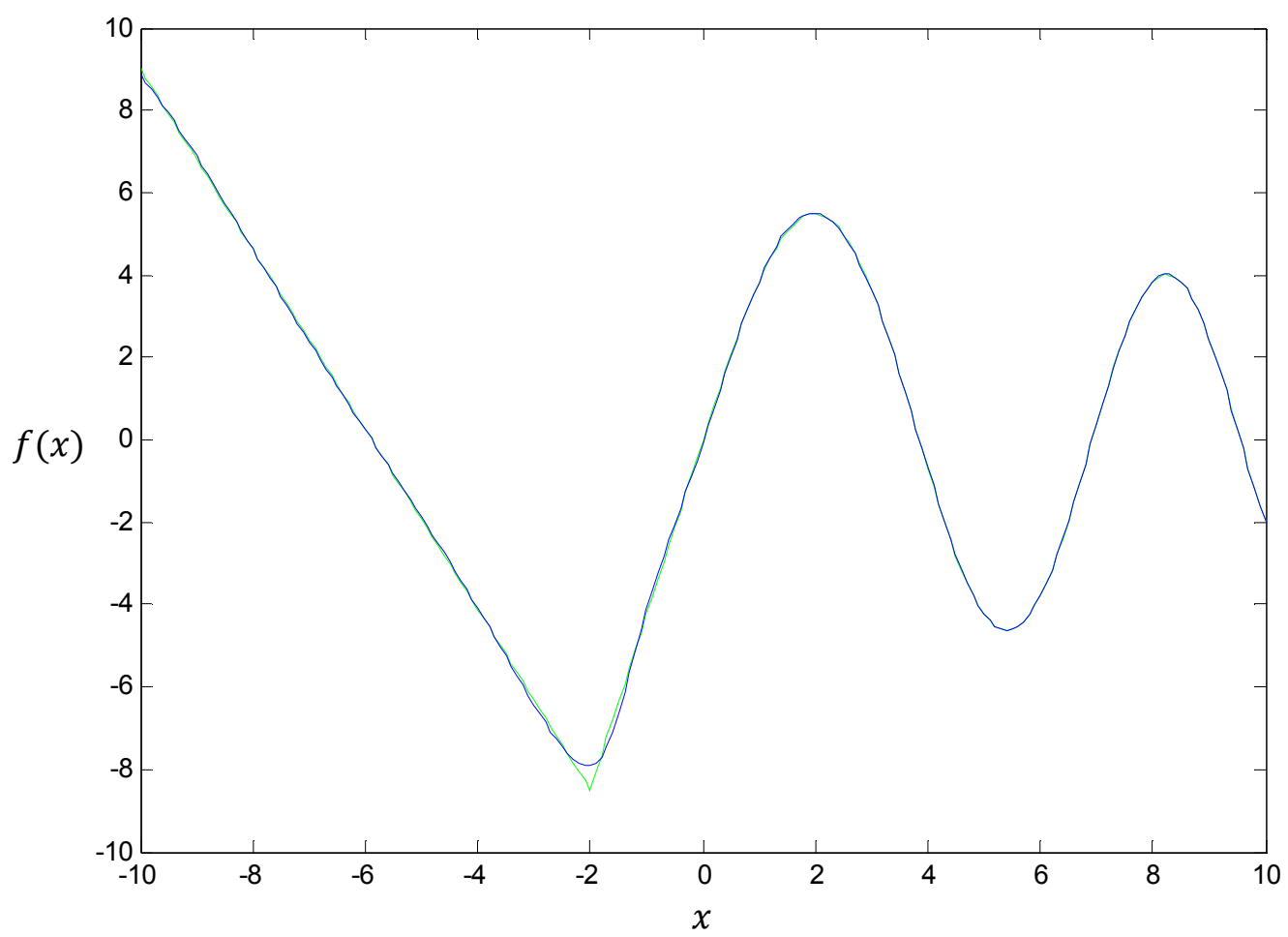

Figure 4.4 Wavelet Neural Network with 7 wavelons trained for 10,000 learning iterations

A Wavelet Neural Network is also simulated to approximate a two-variable function. For this experiment, the selected wavelet is: 


$$
\psi(\tilde{x})=x_{1} x_{2} e^{-\frac{1}{2}\left(x_{1}^{2}+x_{2}^{2}\right)}
$$

The target function is defined by:

$$
f(\tilde{x})=\left(x_{1}^{2}-x_{2}^{2}\right) \sin \left(\frac{1}{2} x_{1}\right)
$$

The original function is shown in figure 4.5 and the resulting approximation is shown in figure 4.6. The model for this structure is $\mathrm{WNN}_{2,49,1}$ and is trained for 40,000 iterations. The number of iteration was determined based numerous trial runs.

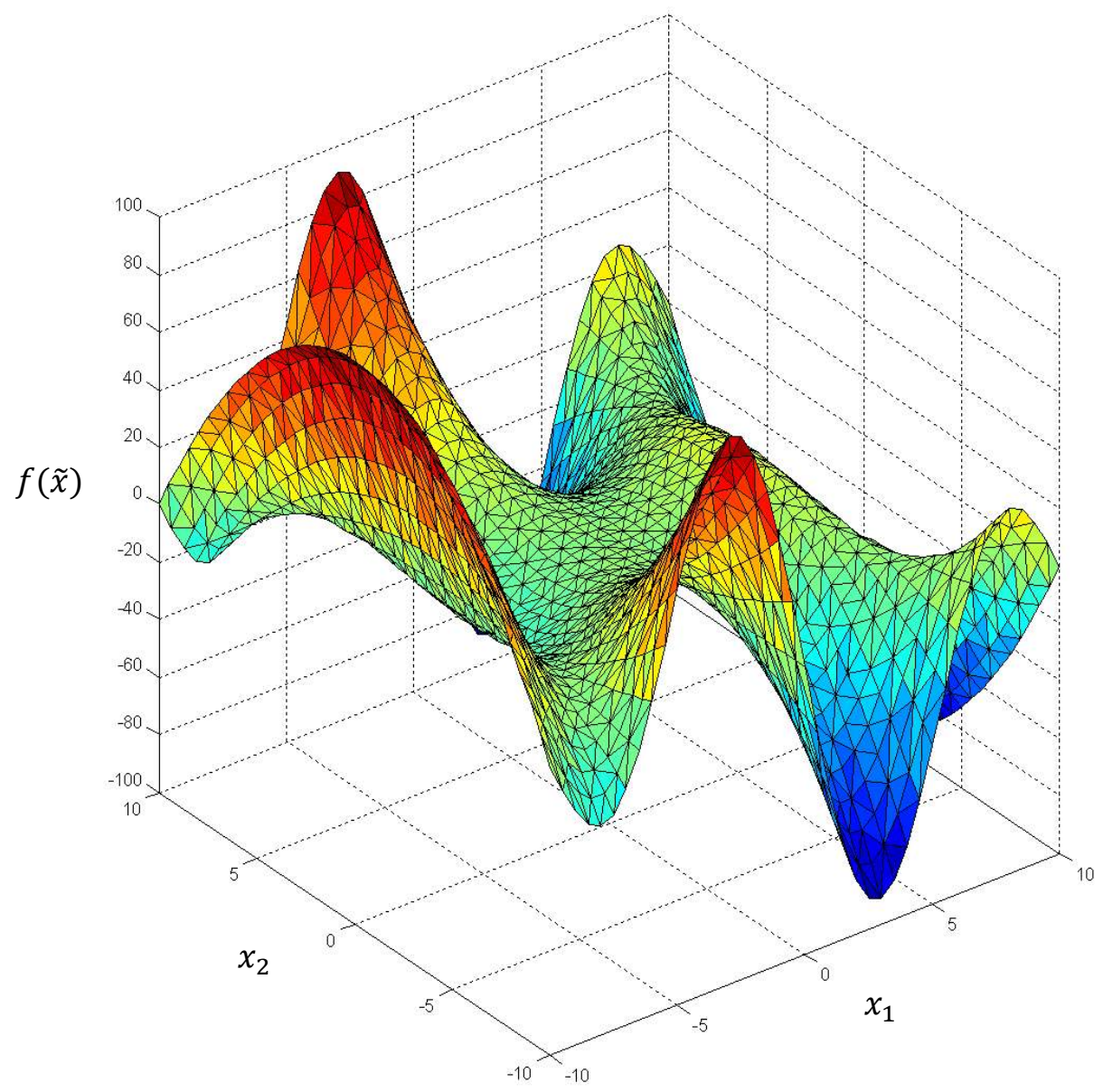

Figure 4.5 The original 3-D function to be approximated by WNN. 


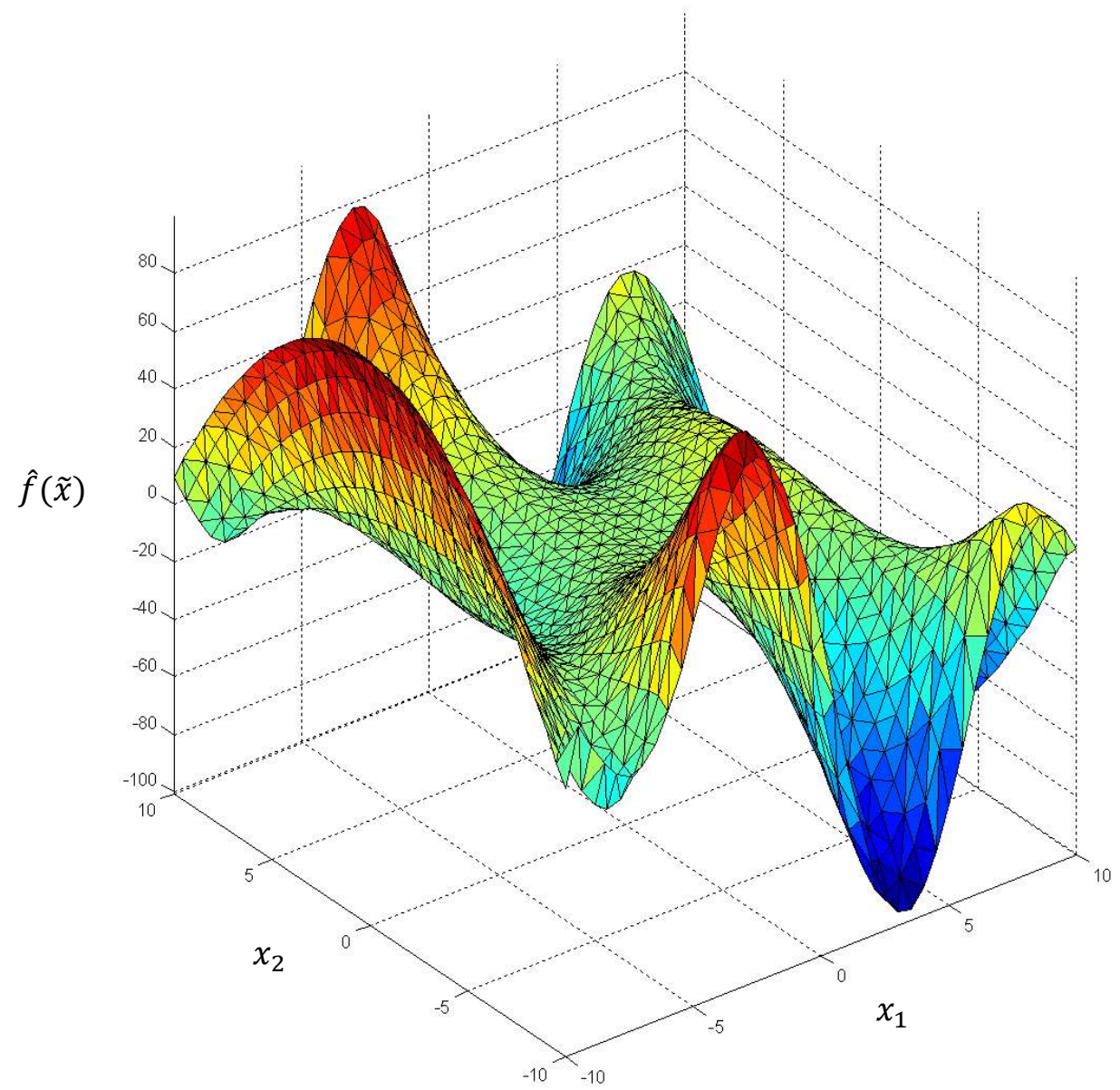

Figure 4.6 An approximated function by WNN after 40,000 iterations.

In addition to the previous results, the experiment is also performed on a function previously shown in chapter 2 (eq. 2.10 with eq. 2.13 as input function). The main purpose of this is to compare the performance of Wavelet Network and Neural Networks. Application for noise reduction is also considered.

In the first test, both Wavelet Network and Neural Network are constrained to have the same number or nearly the same parameters. This is to ensure that one network does 
not have more "memory" over the other. In general, the size of the neural network usually correlates to its capacity to learn and perform tasks. Larger networks have higher numbers of parameters that translate to more "memory". Hence, by keeping the number of the parameter of the two networks to be the same helps to ensure that the comparison is made between design and structure and not between the amounts of memory. The parameters' numbers are determined by a number of adjustable parameters consisting of weights, translations, dilations, and biases. Furthermore, both Neural Networks and Wavelet Neural Networks are given no more than 2,000 learning iterations. From numerous trial runs, this is the near-maximum number of iteration in which the results would not provide further significant improvement if more iteration is given for these particular networks. However, to accommodate for differences in their structural designs, two types of networks are executed at their own suitable learning rate determined from several experimental runs. All the measurements and numerical results are displayed in table 4.1 and figure 4.7-8 provide the graphical comparison. In these figures, the solid green line indicates the original function where the network is trained to learn while the dashed blue line is the networks' approximation. The results in table 4.1 are the typical average measurement from several runs.

Note that the comparison is also made over the multi-layer neural network as well. Because unlike the wavelet neural networks, which consist of only a single hidden layer, neural network's structure can expand to multiple layers, adding more complexity to the model while limited with the same number of parameters. The results are shown in figures 4.9-11. 
Table 4.1 WNN and ANN parameters for system modeling

\begin{tabular}{|c|c|c|c|c|c|}
\hline Method & $\begin{array}{c}\text { Learning } \\
\text { Rate }\end{array}$ & $\begin{array}{c}\text { Hidden } \\
\text { Layer }\end{array}$ & $\begin{array}{c}\text { Number of } \\
\text { Units }\end{array}$ & $\begin{array}{c}\text { Number of } \\
\text { Parameters }\end{array}$ & MSE \\
\hline $\begin{array}{c}\text { Neural } \\
\text { Network }\end{array}$ & 0.25 & 1 & $1-7-1$ & 22 & 0.0157 \\
\hline $\begin{array}{c}\text { Wavelet } \\
\text { Network }\end{array}$ & 0.015 & 1 & $1-7-1$ & 22 & 0.0030 \\
\hline $\begin{array}{c}\text { Neural } \\
\text { Network }\end{array}$ & 0.25 & 2 & $\begin{array}{c}1-3-3-1 \\
1-4-2-1 \\
1-2-4-1\end{array}$ & $\begin{array}{c}22 \\
21\end{array}$ & $\begin{array}{c}0.0105 \\
21\end{array}$ \\
\hline
\end{tabular}

From table 4.1, despite given the same number of parameters, the neural networks failed to perform as well as the wavelet network. There are large gap of means-squareerror (MSE) between the two types of networks. On the other hand, by re-arranging the neural network using two-hidden layer model, its performance is noticeably increased. Because there are many ways in which the network structural can be re-arranged, in this experiment, only three arrangements are used here.

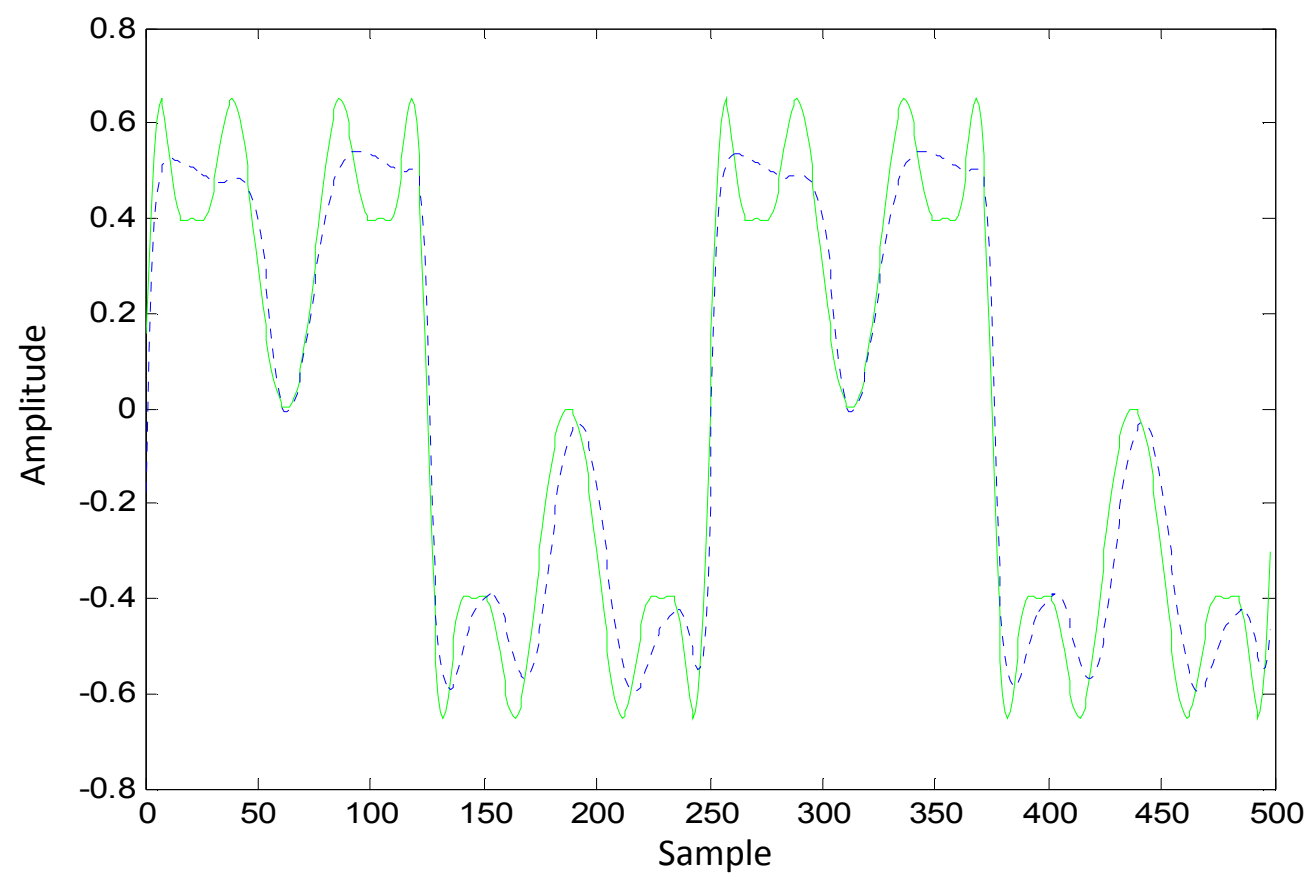

Figure 4.7 Single-layer Artificial Neural Network class N(1-7-1) 


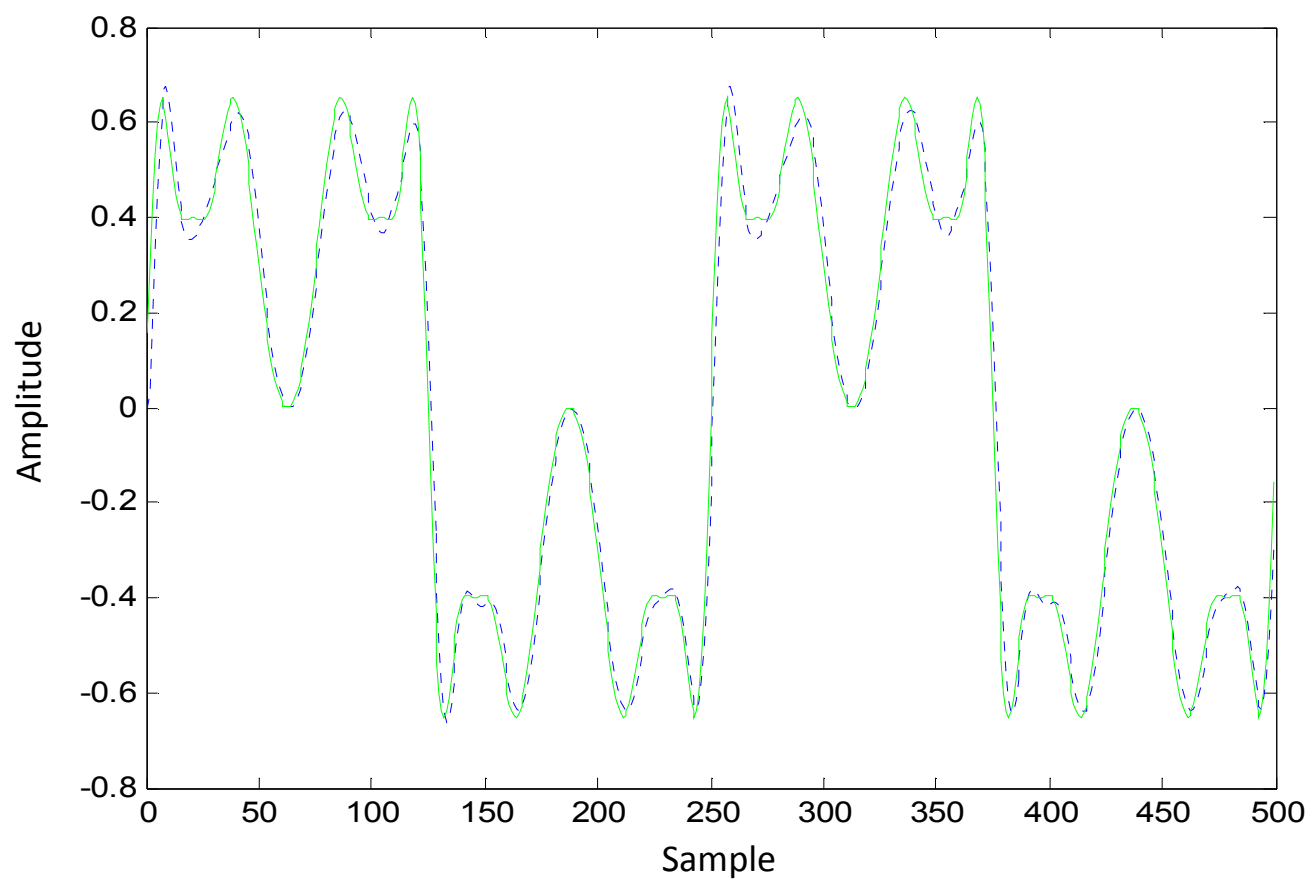

Figure 4.8 Wavelet Neural Network approximation

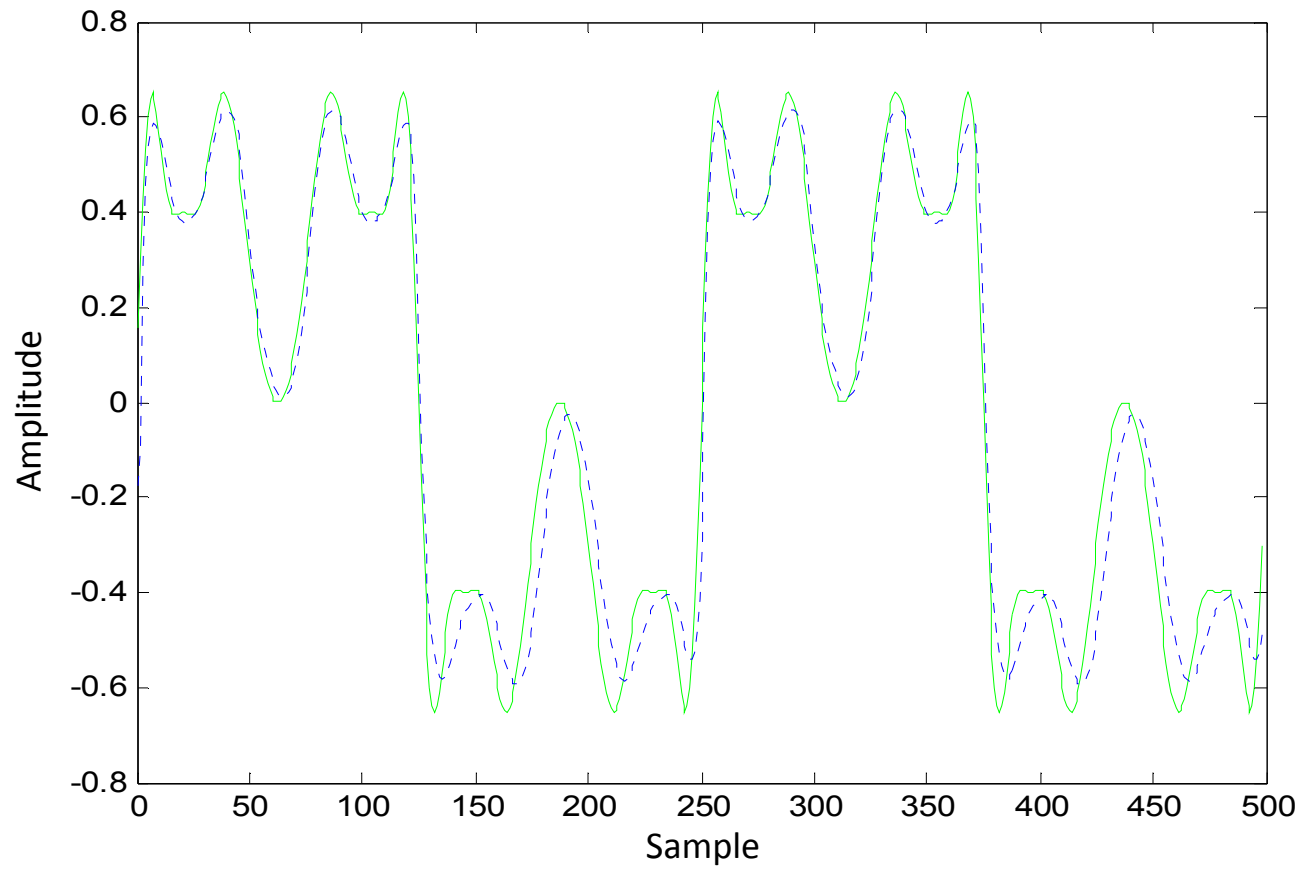

Figure 4.9 Multi-layer Neural Network approximation class N(1-3-3-1) 


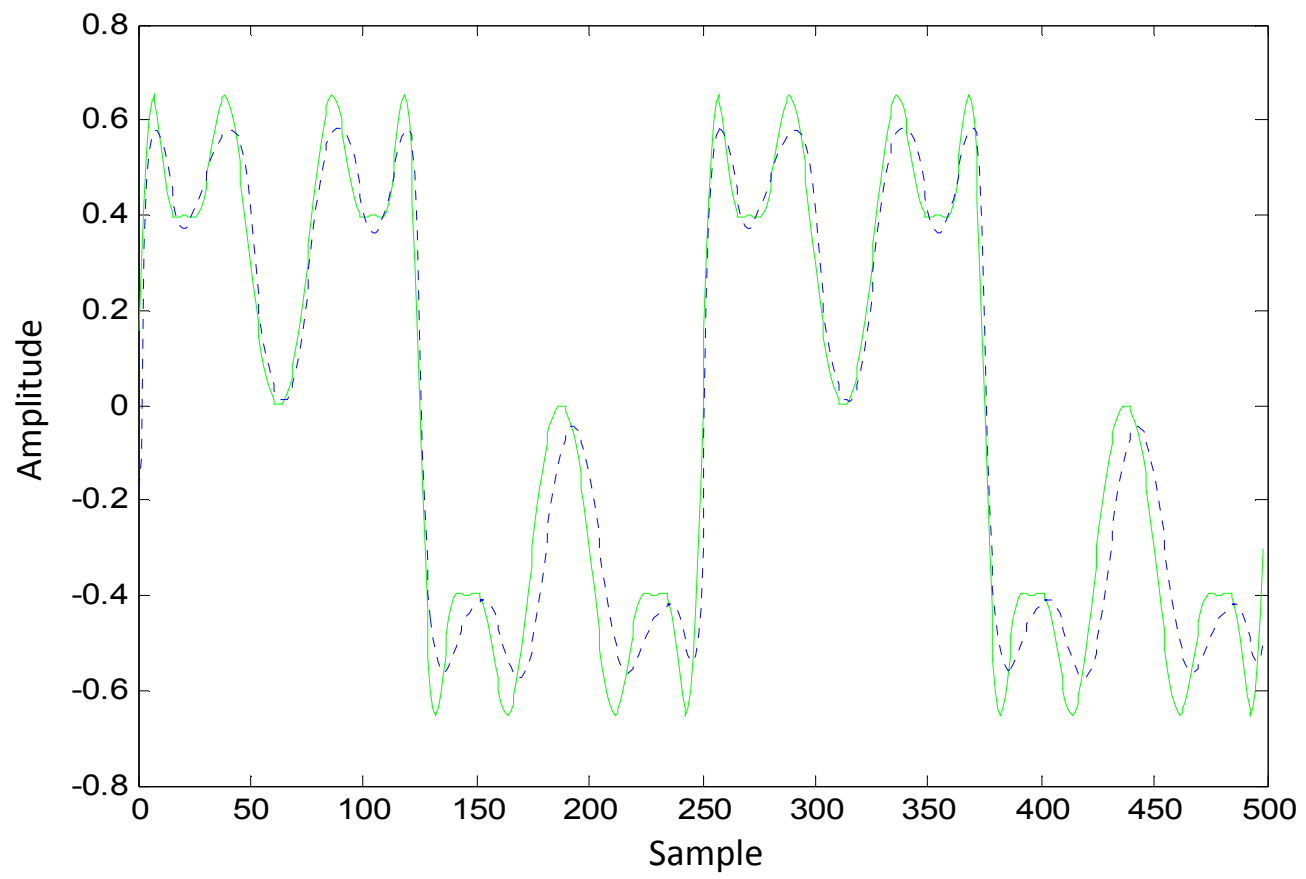

Figure 4.10 Multi-layer Neural Network approximation class N(1-4-2-1)

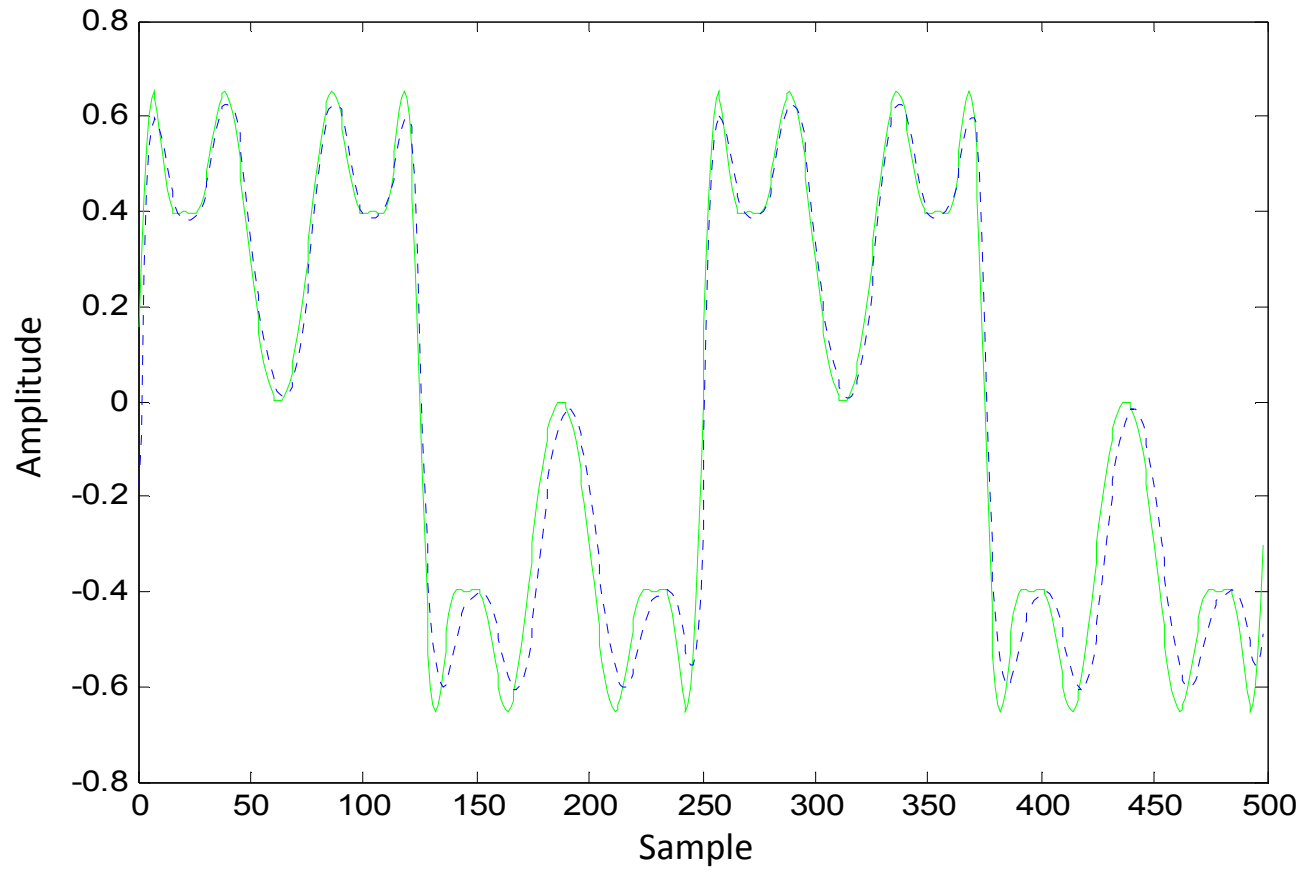

Figure 4.11 Multi-layer Neural Network approximation class N(1-2-4-1) 
In the second test, the overall structure of every network is kept the same. The only difference is the application. The goal of the test is to perform noise reduction over the same equation as the previous test (eq. 2.10). However, instead of a clean input (eq. 2.13), a randomly distributed noise $[-0.25,0.25]$ is added to the input signal. The approximated signal-to-noise ratio (SNR) of the noise at the input is calculated to be $39 \mathrm{~dB}$ and $11 \mathrm{~dB}$ at the output. The networks are trained to provide a noise tolerant characteristic. As noise reduction is a major part of the report, this comparison between both networks can provide a "clue" of how suitable each network is for the application. Once again, the evaluation of the multi-layer neural networks is also being compared here as a result of its improvement over the single layer network. The numerical summary of the experimental result is shown in table 4.2. Figure 4.12-15 provide the graphical results for this test where the green a represents a signal with no contamination at the input, red is the signal with a contaminated input, and blue is a filtered signal. Note that SNR is calculated using equation (4.15) where $A$ is the root mean square (RMS) amplitude [34]:

$$
S N R(d B)=20 \log _{10}\left(\frac{A_{\text {signal }}}{A_{\text {noise }}}\right)
$$

Table 4.2 WNN and ANN parameters for noise reduction

\begin{tabular}{|c|c|c|c|c|c|}
\hline Method & $\begin{array}{c}\text { Learning } \\
\text { Rate }\end{array}$ & $\begin{array}{c}\text { Hidden } \\
\text { Layer }\end{array}$ & $\begin{array}{c}\text { Number of } \\
\text { Units }\end{array}$ & $\begin{array}{c}\text { Number of } \\
\text { Parameters }\end{array}$ & $\begin{array}{c}\text { SNR } \\
\text { (dB) }\end{array}$ \\
\hline Wavelet Network & 0.015 & 1 & $1-7-1$ & 22 & 16.95 \\
\hline Neural Network & 0.25 & 1 & $1-7-1$ & 22 & 18.27 \\
\hline & & & $1-3-3-1$ & 22 & 19.65 \\
Neural Network & 0.25 & 2 & $1-4-2-1$ & 21 & 19.37 \\
& & & $1-2-4-1$ & 21 & 19.00 \\
\hline
\end{tabular}



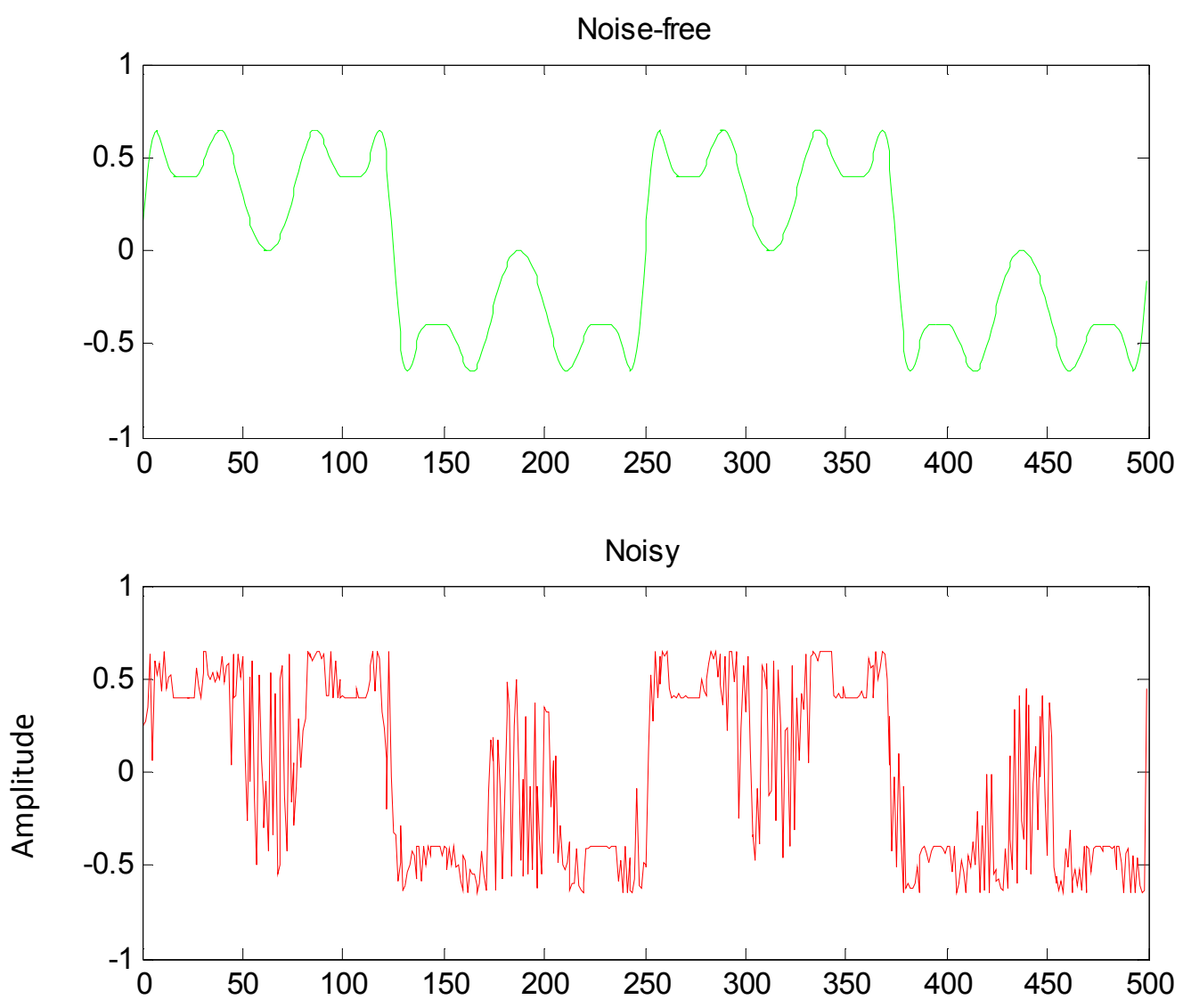

Filtered

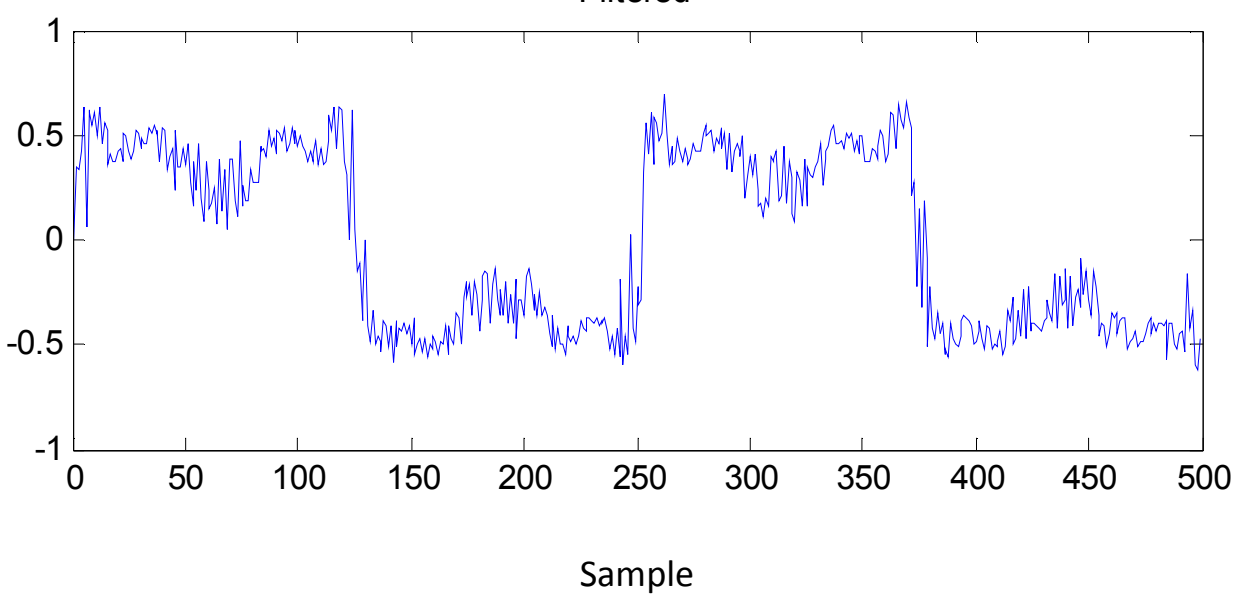

Figure 4.12 Wavelet network class N(1-7-1) 

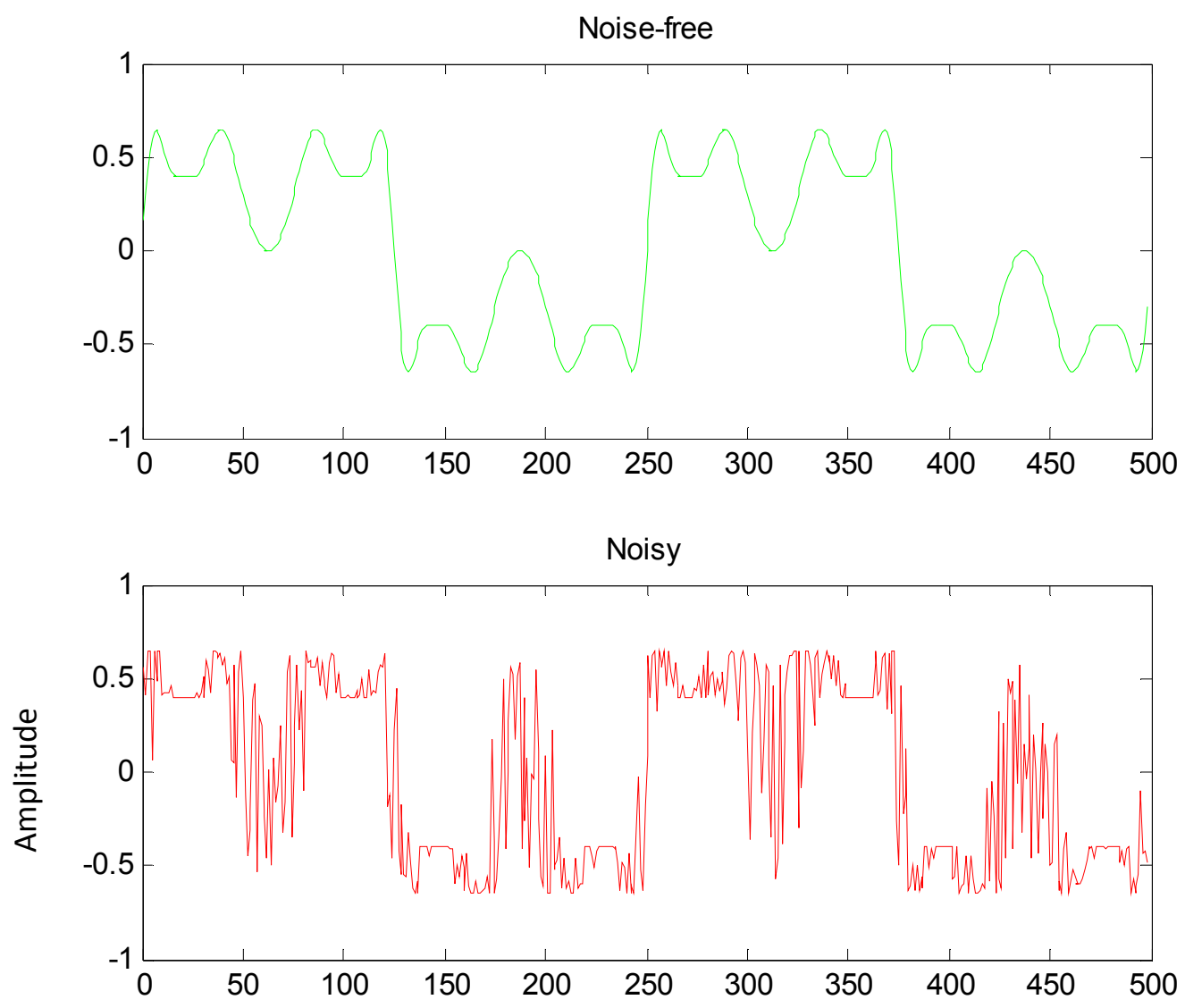

Filtered

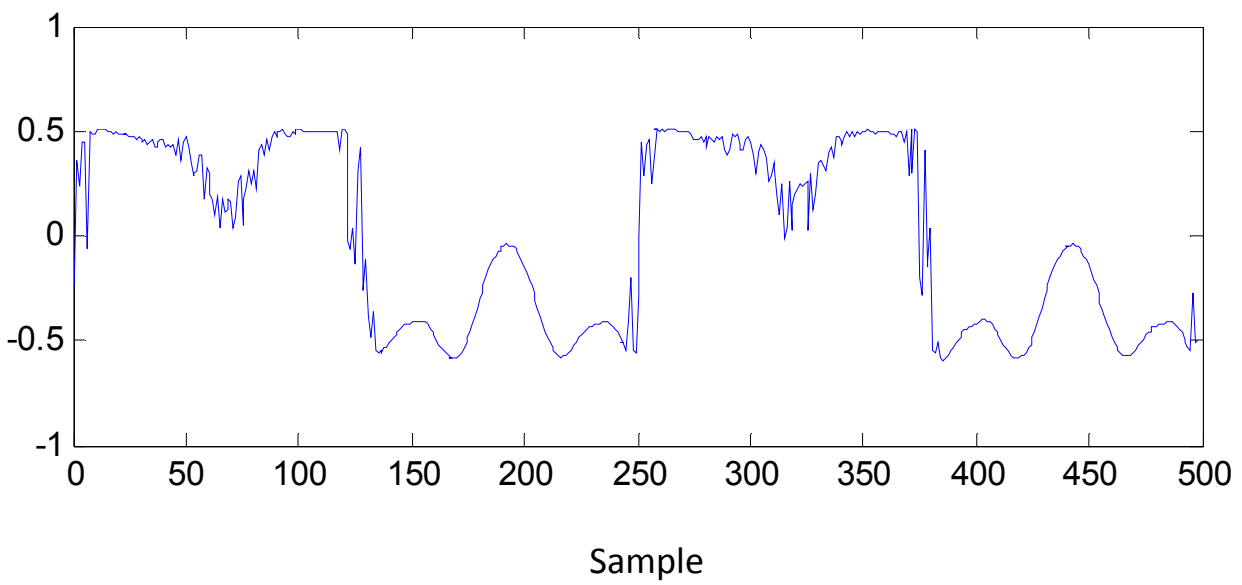

Figure 4.13 Single-layer neural network class N(1-7-1) 

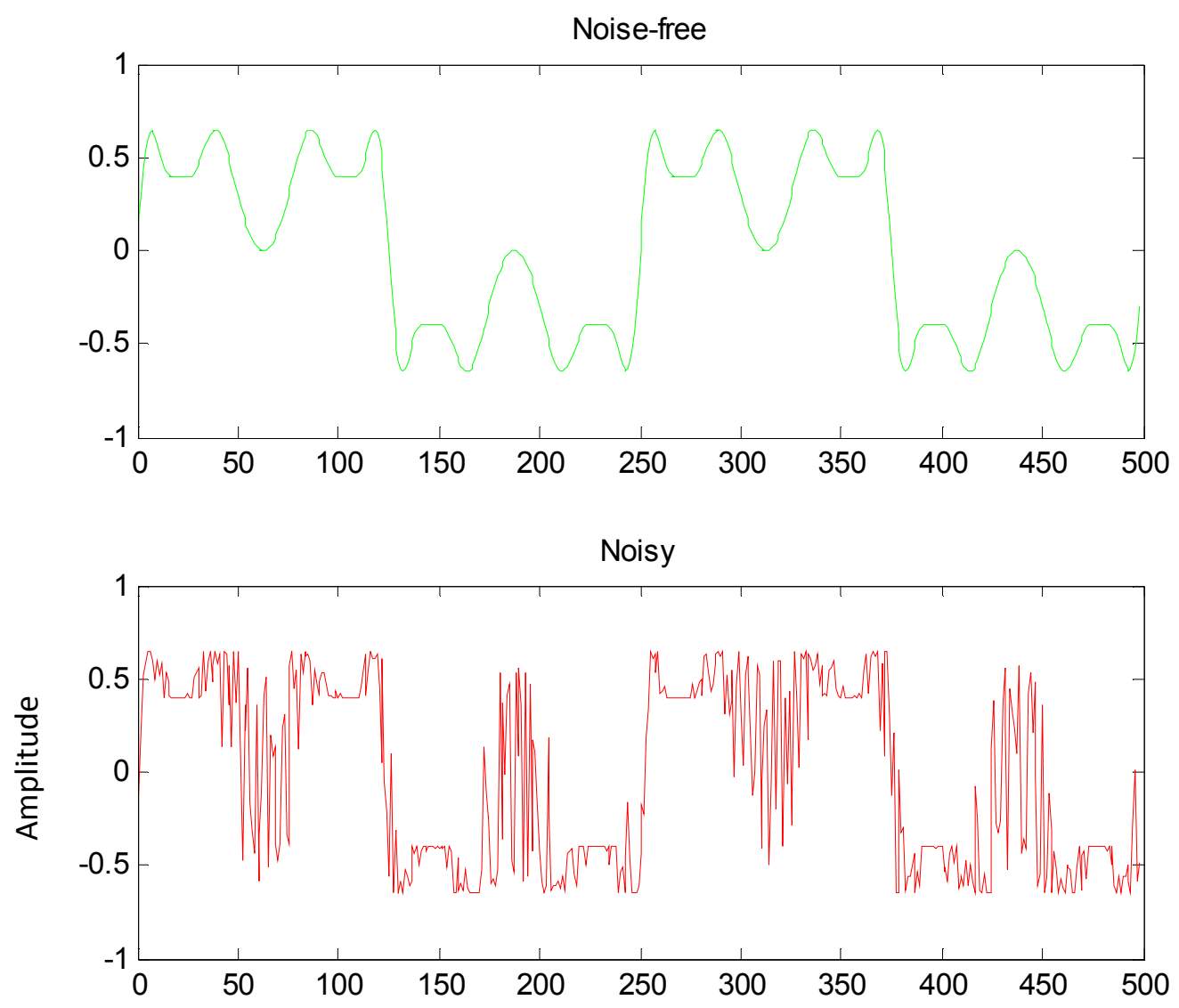

Filtered

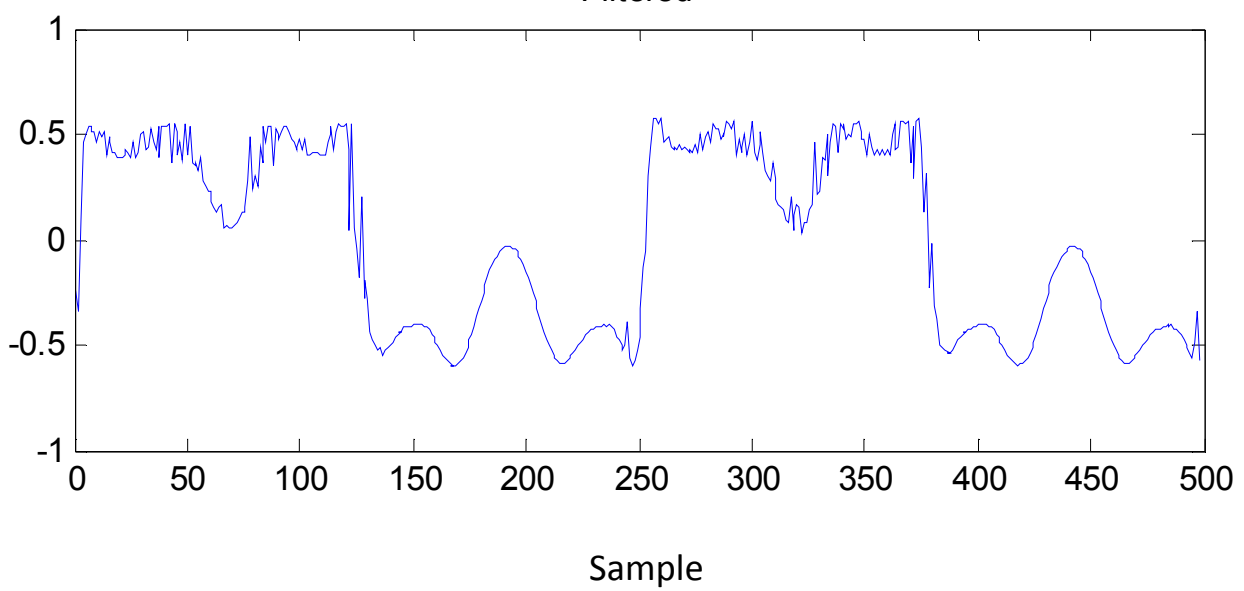

Figure 4.14 Multi-layer neural network class N(1-3-3-1) 

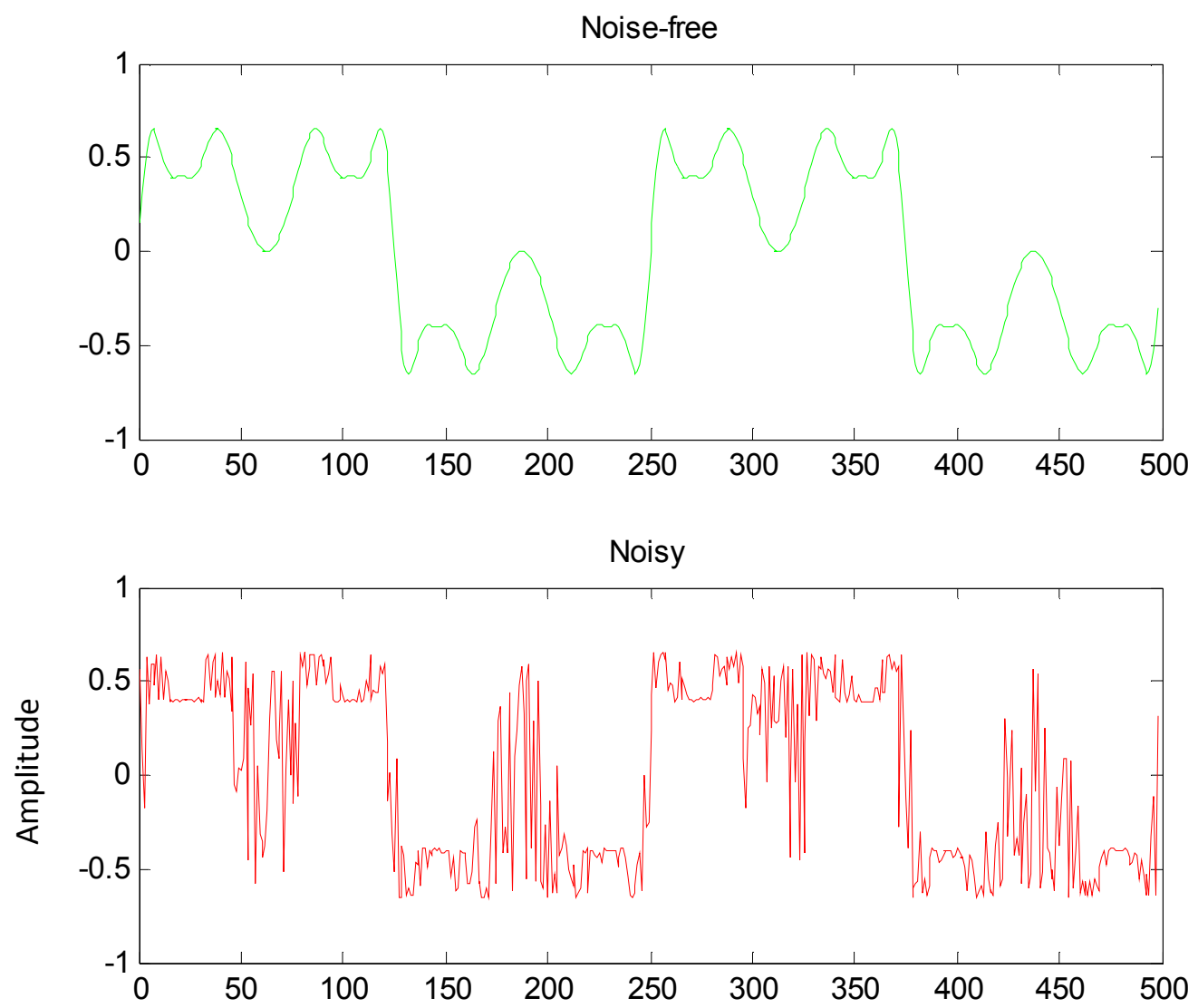

Filtered

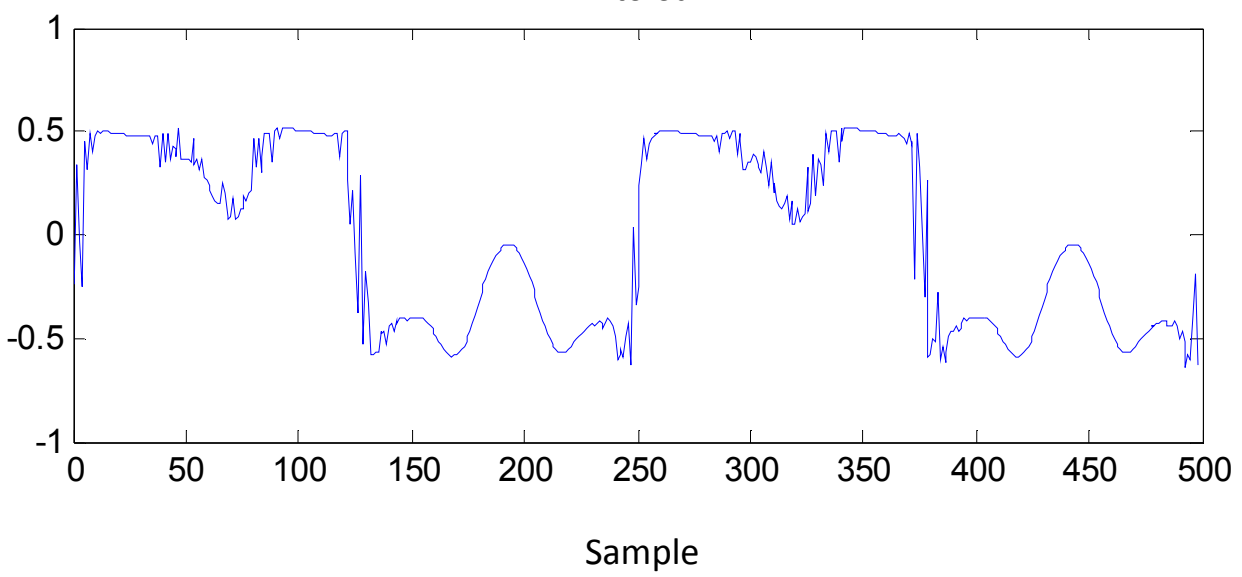

Figure 4.15 Multi-layer neural network class N(1-4-2-1) 

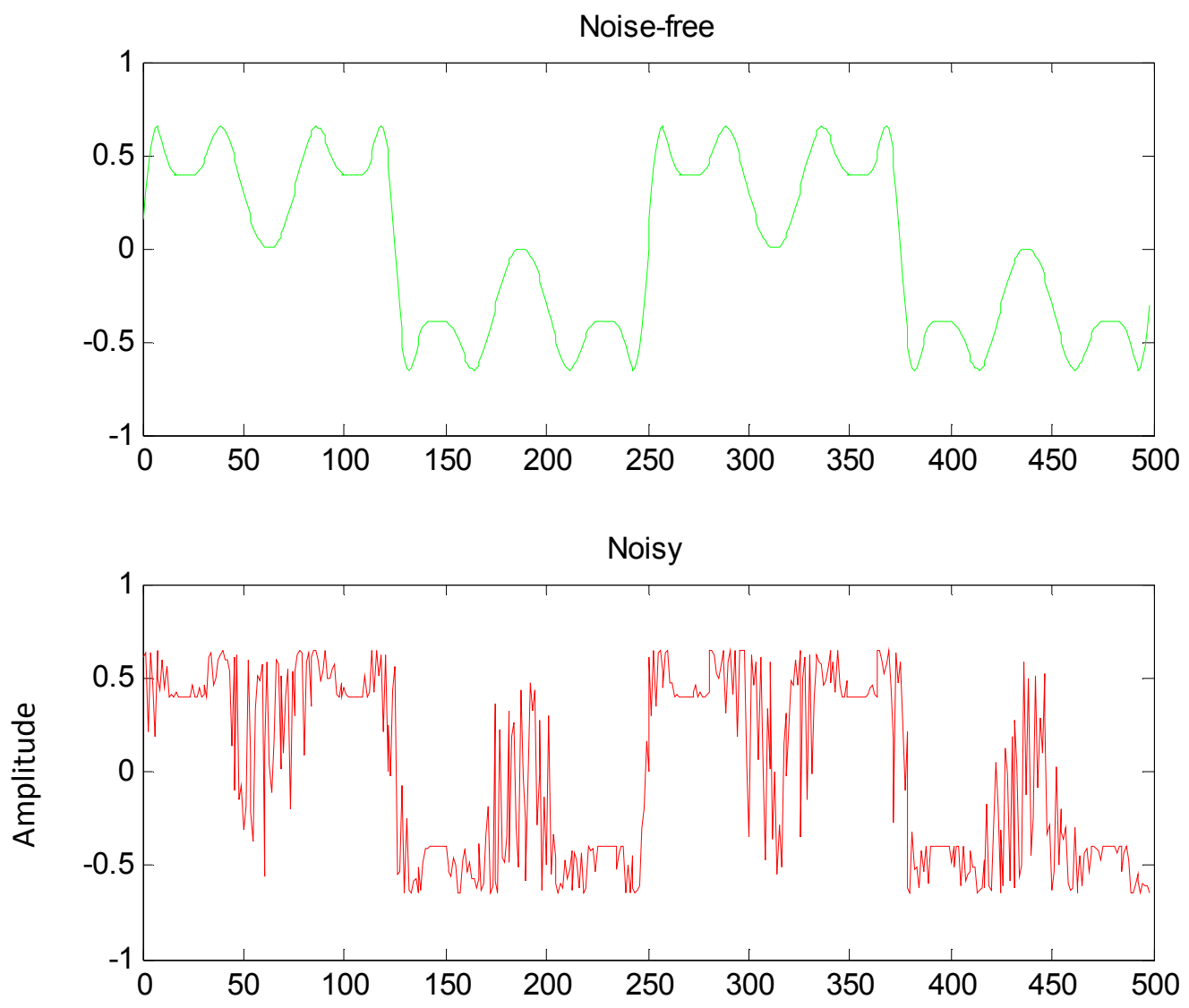

Filtered

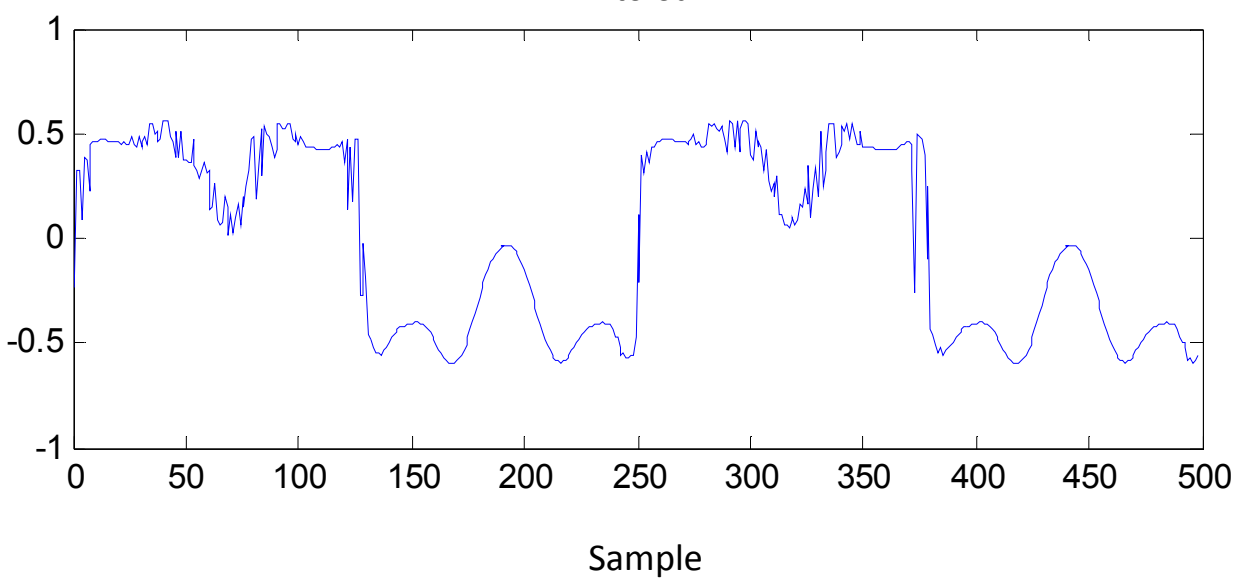

Figure 4.16 Multi-layer neural network class N(1-2-4-1) 


\subsection{Conclusion}

A Wavelet Neural Network, based on the wavelet transform, is a multi-resolution hierarchical artificial neural network, which organically combines the good localization characteristics of the wavelet transform theory and the adaptive learning virtue of neural networks. Its performance is demonstrated through simulations as well as in comparisons with a conventional ANN for system modeling applications from inputoutput observations. From the experiment, WNN is proven to out-perform ANN in system modeling. In order for ANN to further improve in performance, its structure is needed to be re-built into a multi-hidden layer structure adding more complexity into the system. Even so, WNN still performs better. In a second test, the training procedure consists of noise injected into the input. This in-turn motivates the network to become more noise tolerable. Keep in mind that these networks consist of no more than 7 neurons/wavelons in their structure. For this particular task, this is considered insufficient. Hence, good results should not be expected. The point of choosing 7 neurons/wavelons only serves as a test comparison to see how well the networks are able to learn in the situation where resources are insufficient. On the other hand, if sufficient numbers of neurons/wavelons are given, all the networks will be able to learn and perform well. As a result, differences in output margin between networks are small and hard to compare.

Recently, the study of WNN has become an active subject in many scientific and engineering research areas. Different designs as well as various type of learning 
algorithms for WNNs have been proposed in an attempt to improve the performance evermore. In the next chapter, some of these designs will be presented and compared. 


\section{Chapter 5: Learning Algorithms and Design comparison}

Based on wavelet theory, a new notion of the wavelet neural networks has been proposed as an alternative to feed forward neural networks for approximating arbitrary nonlinear functions. Following that, many scholars have recently proposed further improvements upon this existing method. A large number of those designs has proven to be very effective and promising. In this chapter, the comparison between some of those designs will be made based on their learning abilities and accuracies. Because Wavelet Neural Networks are a very active subject, many designs and algorithms have been proposed, and it is not possible to cover all of them in this paper. Hence, only some of the interesting methods will be presented. The design selections are mainly dictated by the author's personal interest. They are mainly optimization algorithms, evolutionary algorithms, fuzzy logic, local linear, and the combination of these. But before these methodologies can be presented, some of the basic understanding of these individual designs is needed. In the following few sections, these concepts will be introduced in brief detail. 


\subsection{Genetic Algorithm}

Genetic Algorithms (GAs) are adaptive search algorithm based on the evolutionary ideas of natural selection and genetic. The basic concept of GAs is designed to simulate processes in a natural system necessary for evolution that follows the principles first laid down by Charles Darwin's "survival of the fittest." As such they represent an intelligent exploitation of a random search within a defined search space to solve a problem.

GA begins with a set of $k$ randomly generated states, called the population. Each state, or individual, is presented as a string over a finite alphabet - most commonly, a string of Os and 1s. Each state is, then, rated by the evaluation function or (in GA terminology) the fitness function. A fitness function should return higher values for better states.

After every state is evaluated, a random choice of pair is selected for reproduction, in accordance with the probabilities. Note that these probabilities are usually set higher for states that return higher fitness values. Some individual may be selected twice usually those with high fitness values - and some may not be selected at all - usually those in the lower range values. For each pair to mate, a crossover point is randomly chosen from the positions in the string.

The offspring themselves are created by crossing over the parent strings at the crossover point. For example, shown in figure 5.1, the first child of the first pair gets the first four strings from the first parent and the remaining string from the second parent, whereas the second child gets the first four strings from second parent and the rest from the first parent. The example illustrates the fact that, when two parent states are 
quite different, the crossover operation can produce quite diverse results during the early process, so crossover frequently takes large steps in the state space early in the search process and smaller steps later on when most individuals are quite similar.

Finally, the offspring are subject to random mutation with a small independent probability. In nature, the mutation occurrence is believed to be a main driving force for evolution. The result of mutation can often result in a weaker individual. Occasionally the result might be to produce a stronger one. In each case, it can be assumed that mutation is doing something new to subtly change some part of the chromosome. The assumption is also made that such changes occur spontaneously and with no reference or affect from other members of the population. In general, mutation is something new that is happening. For GA, such change is also happening here. The most important aspect is that it should occur rarely and that one individual should have its pattern adjusted very slightly. It is also important to remember that mutations should occur very infrequently; otherwise, they will have a disruptive effect on the fitness of the overall population. 


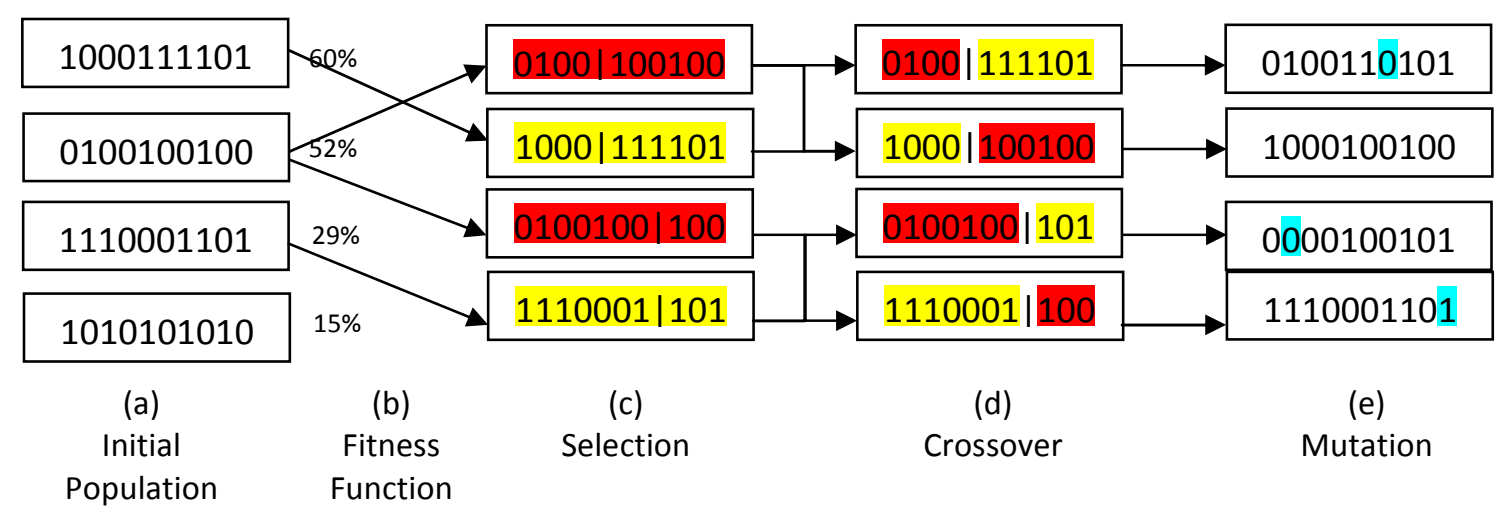

Figure 5.1 The genetic algorithm.

The initial population (a) is ranged by the fitness function in (b), resulting in pairs for mating in (c). They produce offspring in (d), which are subject to mutation in (e).

\subsection{Particle Swarm Optimization}

The Particle Swarm Optimization (PSO) is a population based optimization method first proposed by Kennedy and Eberhart [10] in 1995, inspired by the social behavior of bird flocking or fish schooling. PSO shares many similarities with evolutionary computation techniques such as Genetic Algorithm (GA). The system is initialized with a population of random solutions and searches for optima by updating generations. However, unlike GA, PSO has no evolution operators such as crossover and mutation. In PSO, the potential solutions, called particles, fly through the problem space by following the current optimum particles.

Each particle keeps track of its coordinates, represented by a position vector $z_{i}$, in the problem space which is associated with the best solution (fitness) it has achieved so far. In addition, the fitness value is also stored. This value is called $p_{i}$. When a particle takes 
all the population as its topological neighbors, the best value is a global best and is called $p_{g}$.

The particle swarm optimization concept consists of, at each time step $t$, changing the velocity $v$ of (accelerating) each particle toward its $p_{i}$ location and $p_{g}$ location. Acceleration is weighted by random terms $\left(\phi_{1}\right.$ and $\left.\phi_{2}\right)$, randomly distributed in $[0,1]$, with separate constant numbers $\left(c_{1}\right.$ and $\left.c_{2}\right)$ being generated for acceleration toward $p_{i}$ and $p_{g}$ locations. Change in velocity $v$ can be summarized in equation (5.1):

$$
\begin{aligned}
v_{i}(t+1)=\chi & \left(\alpha v_{i}(t)+c_{1} \phi_{1}\left(p_{i}(t)-z_{i}(t)\right)\right. \\
& \left.+c_{2} \phi_{2}\left(p_{g}(t)-z_{i}(t)\right)\right)
\end{aligned}
$$

where $\chi$ and $\alpha$ are real constants. The parameter $\chi$ controls the magnitude of $v$, whereas the inertial weight $\alpha$ weights the magnitude of the old velocity $v_{i}(t)$ in the calculation of the new velocity $v_{i}(t+1)$. Based on the updated velocities, each particle changes its position according to the following equation:

$$
z_{i}(t+1)=z_{i}(t)+v_{i}(t+1)
$$

Recently, PSO has been successfully applied in many research and application areas. It is demonstrated that PSO gets better results faster and cheaper compared to other methods. Another reason that PSO is attractive is that there are few parameters to adjust. 


\subsection{Adaptive Diversity Learning}

The idea of Adaptive Diversity Learning PSO or ADLPSO was introduced by [14] in hope of avoiding the cluster tendency of the basic PSO. Because of the way basic PSO operates, when the optimum global is found by one particle, the other particles will be drawn toward it. If all particles end up in this optimum, they will stay at this optimum without much chance to escape. The basic idea of this ADL is to allow some of the particles to explore areas of the search space for a possible better solution.

The ADL is actually applied as an additional algorithm to the PSO. That is while some large percentages of the total particles are updated according to basic PSO, the smaller percentages are updated using ADL. According to [14], the proposed diversity model is applied using a specific probability density function (PDF):

$$
p(x)= \begin{cases}(1-q) \beta e^{\beta x}, & x \leq 0 \\ q \beta e^{-\beta x}, & x>0\end{cases}
$$

By solving the PDF inversely, a random diversity search vector $\tilde{d}=\left[d_{1}, d_{2}, \ldots, d_{N}\right]$ :

$$
d_{i}= \begin{cases}\frac{1}{\beta} \ln \left(\frac{r_{i}}{1-q_{i}}\right), & 0<r_{i} \leq\left(1-q_{i}\right) \\ -\frac{1}{\beta}\left(\frac{1-r_{i}}{q_{i}}\right), & \left(1-q_{i}\right) \leq r_{i}<1\end{cases}
$$

where $r_{i}$ is a random real number uniformly distributed between [0, 1]. Adjustable parameters $q_{i} \in[0,1]$ determine the probability of the search direction. The larger the $q_{i}$ is, the higher the search probability in the positive direction. For further information on the characteristic of equation (5.3) and its parameters, refer to [14]. Lastly, the 
control parameter $\beta$, in equation (5.4) is adjusted according to the search process with iteration steps $t$ as follows:

$$
\beta(t+1)=\frac{(\beta(t)-\beta(0)) t}{T}+\beta(0)
$$

A fixed variable $T$ indicates the maximum number of iterations.

Similar to the basic PSO, ADL performs its diversity update using the following rule:

$$
\tilde{z}(t+1)=\tilde{z}(t)+\tilde{d}(t+1)
$$

Where $\tilde{z}(t+1)$ represents the model free parameters at iteration $t+1$ and each element of the vector $\tilde{d}(t+1)$ takes the same form as equation (5.4).

\subsection{Neuro-Fuzzy}

Neural Networks can learn from data, but weights cannot be interpreted directly. They are black boxes to the user. On the other hand, Fuzzy Systems consist of interpretable linguistic rules. But unlike Neural Networks, they cannot learn. Hence, the combination of Neural Networks and Fuzzy Logic form a technique that mutates the human-like reasoning style of fuzzy systems with the learning and connectionist structure of neural networks. Figure 5.2 displays a Neuro-Fuzzy Network with all its basic layers. Note that, because the focus of this paper is Wavelet Neural Network, the topic of Neuro-Fuzzy will not be discussed in greater detail here. The concept will only be briefly introduced to the readers, so that the readers can develop sufficient understand necessary for the following section. For further information on this specific topic, refer to [18], [19]. 


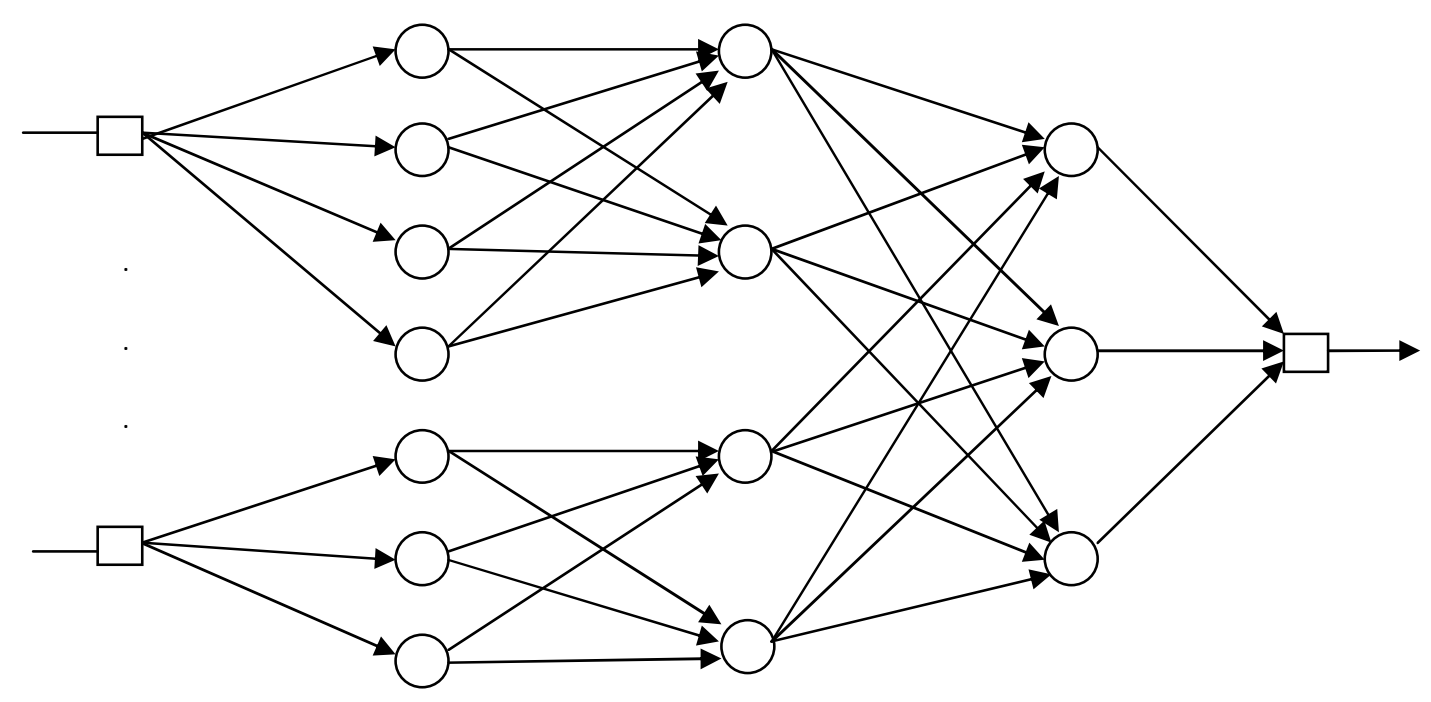

\begin{tabular}{|c|c|c|c|c|c|c|c|}
\hline Input & Antecedent & Weight & Rule & Weight & Consequent & Weight & Output \\
\hline Layer 1 & Layer 2 & Layer 3 & Layer 4 & Layer 5
\end{tabular}

Figure 5.2 Basic Neuro-Fuzzy Network Structure

The Neuro-Fuzzy model consists of five layers feed forward network, which is comprised by the input, diffizification, rule, composite, and output layer.

The First Layer - The first layer is the input layer, where every node corresponds to one of the input variables.

The Second Layer - This is the diffizification layer and consists of input membership function of the antecedent and their weights. The antecedent performs somewhat like the IF-statement (ie. IF X is A).

The Third Layer - In this layer, only the activated nodes of the diffizification are linked which referred to as being "fired" and the value at the end of each rule represents the 
initial weight of the rule that will be adjusted to its appropriate level at the end of training.

The Fourth Layer - The fourth layer is a composite layer. Here, each neuron represents a consequent proposition; its membership function can be implemented by combining one or two sigmoid functions and linear functions. In a simple term, one can think of this layer as the THEN-statement layer of the second layer (ie. THEN Y is B).

The Fifth Layer - The last layer simply combines all the outputs that are fired from the fourth layer. The output of the fifth layer is the output of the whole network.

\subsection{Wavelet Neuro-Fuzzy}

In the previous section, Neuro-Fuzzy methods were introduced to provide some basic knowledge and background on the subject. In this section, the discussion will be more specific to the Wavelet Neuro-Fuzzy model. For the implementation presented in this paper, the structural design will follow the Generalized Wavelet Neuro-Fuzzy model proposed by [17].

Figure 5.3 shows a wavelet neuro-fuzzy model. The output of this model is defined by equation (5.7):

$$
\hat{Y}=\frac{a}{b}=\frac{\sum_{j=1}^{M}\left(\mu_{j} \cdot \hat{Y}_{W N N_{j}}\right)}{\sum_{j=1}^{M} \mu_{j}}
$$


where $\hat{Y}_{W N N}$ is output of a wavelet neural network and $\mu_{j}$ is output rule's firing strength calculated by equation (5.8):

$$
\mu_{j}=\prod_{i=1}^{n} e^{-\left(\frac{x_{i}-\bar{x}_{j, i}}{\sigma_{j, i}}\right)^{2}}
$$

The parameters $\bar{x}_{j, i}$ and $\sigma_{j, i}$ are the mean and standard deviation, respectively, of the $\mathrm{j}^{\text {th }}$ term associated with $\mathrm{i}^{\text {th }}$ input variable $x_{i}$. Parameter $M$ is the number of rules and $n$ is number of input dimensions. For the sake of clarification, the output of the wavelet neural network in equation (5.7) is re-written in the form of:

$$
\widehat{Y}_{W N N_{j}}=\sum_{s=1}^{M} \sum_{\tau=0}^{s} w_{s, \tau, j} \Psi_{s, \tau}\left(\sum_{j=1}^{n} C_{s, \tau}^{i} \cdot x_{i}\right)
$$

Note that instead of defining resolution parameters of wavelet as translation and dilation, a single variable $C_{s, t}^{i}$ is used instead.

For the model presented here, the adjustable learning parameters are $\bar{x}, \sigma, C$, and $w$ by using least square error algorithm:

$$
E=\frac{1}{2 P} \sum_{p=1}^{P}(\hat{Y}-y(p))^{2}
$$

Hence, the adjustment for each parameter is defined by

$$
\begin{aligned}
\sigma_{i, j}(t+1) & =\sigma_{i, j}(t)-\eta \frac{d E}{d \sigma_{i, j}} \\
& =\sigma_{i, j}(t)-\eta \frac{1}{P}(\hat{Y}-y(p)) \frac{d \hat{Y}}{d \sigma_{i, j}}
\end{aligned}
$$




$$
\begin{aligned}
=\sigma_{i, j}(t)-\eta & \frac{1}{P}(\hat{Y}-y(p)) \frac{d \hat{Y}}{d \mu_{j}} \frac{d \mu_{j}}{d \sigma_{i, j}} \\
=\sigma_{i, j}(t)-\eta & \frac{1}{P}(\hat{Y} \\
& \quad-y(p)) \frac{d \hat{Y}}{d \mu_{j}}\left[\left(\prod_{\substack{i=1 \\
i \neq j}}^{n} e^{-(z)^{2}}\right)\left(\frac{2}{\sigma_{j, i}}(z)^{2} e^{-(z)^{2}}\right)\right]
\end{aligned}
$$

$$
\begin{aligned}
\bar{x}_{i}(t+1) & =\bar{x}_{i}(t)-\eta \frac{d E}{d \bar{x}_{i}} \\
& =\bar{x}_{i}(t)-\eta \frac{1}{P}(\hat{Y}-y(p)) \frac{d \hat{Y}}{d \bar{x}_{i}} \\
& =\sigma_{i, j}(t)-\eta \frac{1}{P}(\hat{Y}-y(p)) \frac{d \hat{Y}}{d \mu_{j}} \frac{d \mu_{j}}{d \bar{x}_{i}} \\
& =\sigma_{i, j}(t)-\eta \frac{1}{P}(\hat{Y}-y(p)) \frac{d \hat{Y}}{d \mu_{j}}\left[\left(\prod_{\substack{i=1 \\
i \neq j}}^{n} e^{-(z)^{2}}\right)\left(\frac{2}{\sigma_{j, i}}(z) e^{-(z)^{2}}\right)\right]
\end{aligned}
$$

$$
\begin{aligned}
w_{s, \tau}^{j}(t+1) & =w_{s, \tau}^{j}(t)-\eta \frac{d E}{d w_{s, \tau}^{j}} \\
& =w_{s, \tau}^{j}(t)-\eta \frac{1}{P}(\hat{Y}-y(p)) \frac{d \hat{Y}}{d w_{s, \tau}^{j}} \\
& =w_{s, \tau}^{j}(t)-\eta \frac{1}{P}(\hat{Y}-y(p)) \frac{d \hat{Y}}{d \hat{Y}_{W N N_{j}}} \frac{d \hat{Y}_{W N N_{j}}}{d w_{s, \tau}^{j}} \\
& =w_{s, \tau}^{j}(t)-\eta \frac{1}{P}(\hat{Y}-y(p)) \frac{d \hat{Y}}{d \hat{Y}_{W N N_{j}}}\left[\Psi_{s, \tau}\left(\sum_{i=1}^{n} C_{s, \tau}^{i} x_{i}\right) x_{j}\right] \\
C_{s, \tau}^{j}(t+1) & =C_{s, \tau}^{j}(t)-\eta \frac{d E}{d C_{s, \tau}^{j}} \\
& =C_{s, \tau}^{j}(t)-\eta \frac{1}{P}(\hat{Y}-y(p)) \frac{d \hat{Y}}{d C_{s, \tau}^{j}} \\
& =C_{s, \tau}^{j}(t)-\eta \frac{1}{P}(\hat{Y}-y(p)) \frac{d \hat{Y}}{d \hat{Y}_{W N N_{j}}} \frac{d \hat{Y}_{W N N_{j}}}{d C_{s, \tau}^{j}} \\
& =C_{s, \tau}^{j}(t)-\eta \frac{1}{P}(\hat{Y}-y(p)) \frac{d \hat{Y}}{d \hat{Y}_{W N N_{j}}}\left[w_{s, \tau}^{j} \Psi_{s, \tau}^{\prime}\left(\sum_{i=1}^{n} C_{s, \tau}^{i} x_{i}\right) x_{i}\right]
\end{aligned}
$$


where $\eta$ is an adaptive learning parameter and $z, \frac{d \hat{Y}}{d \mu_{j}}, \frac{d \widehat{Y}}{d \hat{Y}_{W N N_{j}}}$ are defined by

$$
\begin{aligned}
z & =\frac{x_{i}-\bar{x}_{j, i}}{\sigma_{j, i}} \\
\frac{d \hat{Y}}{d \mu_{j}} & =\frac{\left(\hat{Y}_{W N N_{j}}\right)\left(\sum_{j=1}^{M} \mu_{j}\right)-\left(\sum_{j=1}^{M} \mu_{j} \hat{Y}_{W N N_{j}}\right)}{\left(\sum_{j=1}^{M} \mu_{j}\right)^{2}} \\
\frac{d \hat{Y}}{d \hat{Y}_{W N N_{j}}} & =\frac{\mu_{j}}{\sum_{j=1}^{M} \mu_{j}}
\end{aligned}
$$

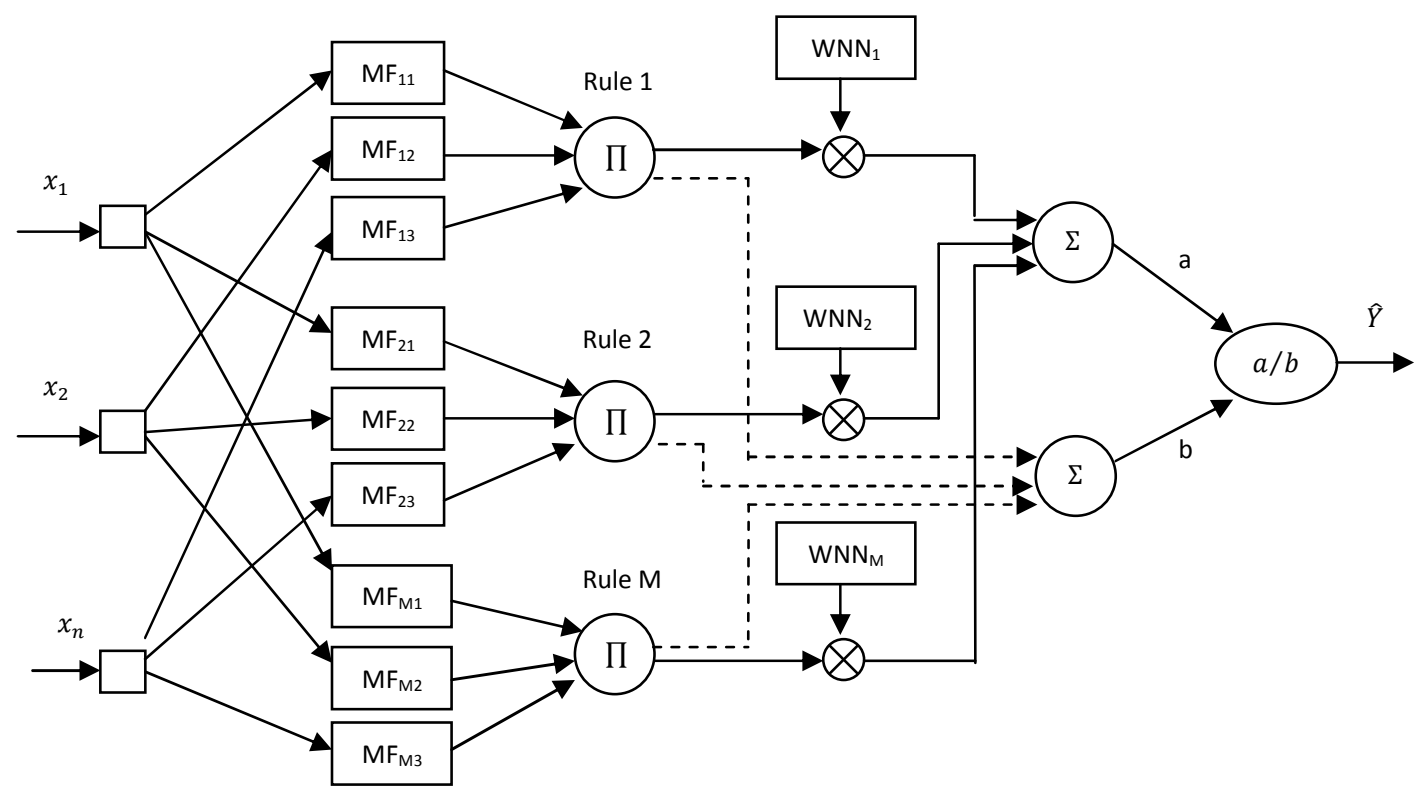

Layer 1

Layer 2

Layer 3

Layer 4

Layer 5

Figure 5.3 Generalized Neuro-Fuzzy model

\subsection{Local Linear Structure Implemented Wavelet Neural Network}

The local linear model for WNN (LLWNN) was proposed by [14] in which the connection weights between hidden layer units and output units are replaced by a local linear 
model. By doing so, this particular network will gain advantage over the traditional design in high dimensional as well as more complex problems. In traditional designs of ANN and WNN alike, the complexity of the network increases as the dimensions of the input increases. Because a network with higher hidden units provides larger capacity necessary to handle large input dimensions, higher dimensional problems usually translate to an increase in the number of the hidden units. Hence, taking the advantage of the local capacity by implementing local linear models will help to keep the number of hidden units down while providing larger capacity. The architecture of the LLWNN is shown in figure 5.4 . Its output is given by:

$$
y=\sum_{i=1}^{N} v_{i} \Psi_{i}(\tilde{x})
$$

A variable $v_{i}$ is a replacement of the traditional weight $w_{i}$. Instead, it consists of a piecewise constant model:

$$
v_{i}=w_{i, 0}+w_{i, 1} x_{1}+\cdots+w_{i, n} x_{n}
$$

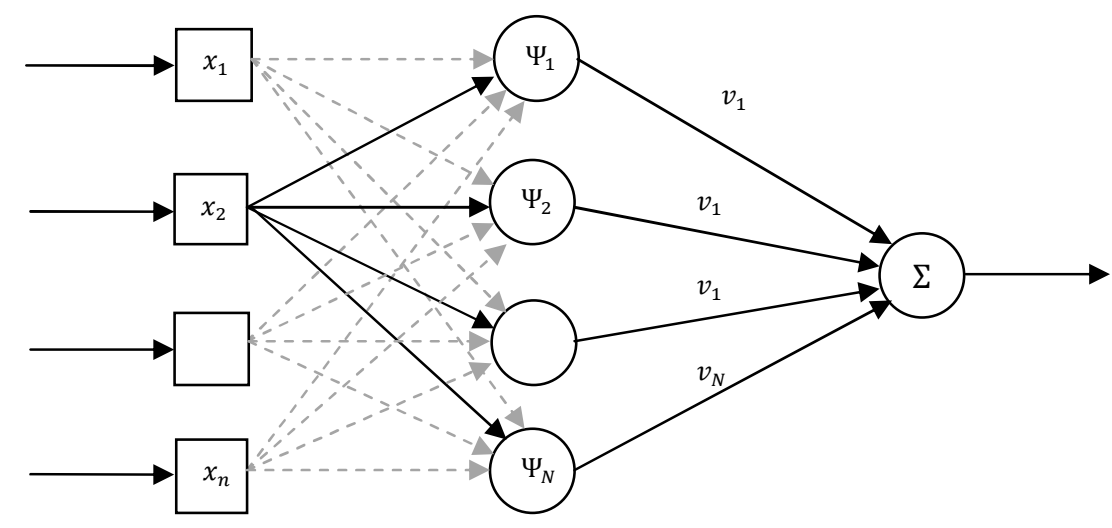

Figure 5.4 Basic wavelet neural network structure 


\subsection{System Models Comparison}

In this section, comparison of the models previously described will be presented. For the models' evaluation, these WNNs will simulate a piecewise function of equation (4.12) previously used in chapter 4.

$$
f(x)=\left\{\begin{array}{lr}
-2.186 x-12.864, & -10 \leq x<-2 \\
4.246 x, & -2 \leq x<0 \\
10 e^{-0.05 x-0.5} \sin ((0.03+0.7) x), & 0 \leq x \leq 10
\end{array}\right.
$$

The objective performance benchmark includes the approximation accuracy property, convergence speed as well as the quality of approximation proportional to the network's size. Note that, the accuracy of model approximation depends highly on the adjustable learning parameters. Therefore, in order to provide a valid comparison, the number of the parameters for each model is kept within a comparison range of one another. Additionally, the learning rate for all models is set to 0.02 with 2,000 iterations.

$$
\psi(x)=-x e^{-\frac{1}{2} x^{2}}
$$

A Gaussian-derivative wavelet in equation (4.11) is used for all networks in this experiment with exception to GMM-WWN (formal introduction will be introduced shortly) where an addition Mexican Hat wavelet is also being used. The Mexican Hat wavelet is characterized by equation (5.20).

$$
\psi(x)=\left(1-x^{2}\right) e^{-\frac{x^{2}}{2}}
$$


Brief introduction of the methods and their parameters are as following.

Genetic Wavelet Neural Network (GWNN) - To battle with the problem of local minima for neural network learning with gradient decent algorithm, Genetic Algorithm is introduced for finding a general solution before fine-tuning with gradient decent. For this particular experiment, all the constant parameters are listed in table 5.1.

Table 5.1 GWNN and GMM-WNN parameter setting

\begin{tabular}{|l|l|}
\hline Number of population & 20 \\
\hline Genetic Learning & $20 \%$ of total iteration \\
\hline Bit Resolution & 16 (2's complement) \\
\hline Mutation Percentage & $20 \%$ \\
\hline Additional Rules: \\
\hline Top 40\% are guarantee for cross-over \\
\hline Bottom 20\% will automatically die out \\
\hline
\end{tabular}

GWNN Implementing Multiple Mother Wavelets and Hybrid Gradient Learning (GMMWNN) - Information consists of signals which are made up of different patterns that change in different time slots. Therefore, by introducing an additional mother wavelet to the system, neural networks will be able to determine the suitability of the mother wavelets on different sections of the signals for better representation. Because of this the idea of the implemented mother wavelets is applied. GMM-WNN's parameters are the same as GWNN. See table 5.1.

Local Linear Wavelet Neural Network (LLWNN) - The local linear model added to the WNN will help to increase the network's capacity while maintaining a small size structure. 
Local Linear with Hybrid ADLPSO and Gradient Learning (ADLPSO-LLWNN) - To battle with the problem of local minima for neural network learning with gradient decent algorithm, ADLPSO is introduced as swarm's search looking for an optimal solution at multiple points. ADLPSO-LLWNN's parameters are defined in table 5.2:

Table 5.2 ADLPSO-LLWNN parameter setting

\begin{tabular}{|l|l|}
\hline Number of population & 20 \\
\hline $\mathrm{B}(0)$ & 0.05 \\
\hline $\mathrm{B}(1)$ & 0.4 \\
\hline$\alpha$ & 0.7 \\
\hline $\mathrm{c} 1=\mathrm{c} 2$ & 2.0 \\
\hline$\chi$ & 0.8 \\
\hline ADLPSO Learning & $20 \%$ of total iteration \\
\hline PSO population & Top $70 \%$ of population \\
\hline ADL population & Bottom $30 \%$ of population \\
\hline
\end{tabular}

Wavelet Neuro-Fuzzy Network (WNFN) - The fuzzy model provides the advantage of a human-like knowledge base of fuzzy inference. A fuzzy logic system has been shown to arbitrarily approximate any nonlinear function and has been successfully applied to system modeling. Due to the fact that WNFN structure is very large, for the implementation in the following section, the number of networks is limited to keep the number of parameters within a certain range. Here, the number of rule is equal to the number of network which is 2 .

Conventional Wavelet Neural Network (WNN) - Lastly, traditional WNN will also be compared against the above models to get perspectives of the scale of the improvement for each model. 
Table 5.3 shows the approximation results obtained with the six methods. As you can see, except for WNFN, all networks have made improvement upon WNN. Once again, the numbers of parameters for each method are kept within a compatible range of one another. As a result, WNFN is put into disadvantage due to its large structure.

The implementation of multiple wavelet of GMM-WNN shows large improvement over GWNN. It also has the best match approximation of all. Another outstanding method is ADLPSO-LLWNN with figure of merit (calculated using simple average error) almost as low as GMM-WNN. GMM-WNN and ADLPSO-LLWNN are the two best performers of all these methods. However, the evaluation is also based on time. Though the two methods may have very close approximation; ADLPSO-LLWNN took almost half of the time of GMM-WNN. A study of error function in figure 5.5 also shows that LLWNN has a much quicker convergent rate even at low iterations. A normal WNN also has a good convergent rate at the beginning. But its convergence rate is much smaller as the number of iterations get higher. This may be due to the problem of local minima.

Table 5.3 Simulation result from all models and their parameters comparison

\begin{tabular}{|c|c|c|c|c|c|}
\hline Method & $\begin{array}{c}\text { Number of } \\
\text { Wavelons }\end{array}$ & $\begin{array}{c}\text { Number of } \\
\text { Networks }\end{array}$ & $\begin{array}{c}\text { Number of } \\
\text { Parameters }\end{array}$ & $\begin{array}{c}\text { Training } \\
\text { Time (s) }\end{array}$ & $\begin{array}{c}\text { Figure } \\
\text { of Merit }\end{array}$ \\
\hline WNN & 6 & 1 & 19 & 3.718 & 0.1368 \\
\hline GWNN & 6 & 20 & 19 & 6.382 & 0.0668 \\
\hline GMM-WNN & 6 & 20 & 19 & 7.905 & 0.0374 \\
\hline LLWNN & 5 & 1 & 20 & 2.656 & 0.0554 \\
\hline $\begin{array}{c}\text { ADLPSO- } \\
\text { LLWNN }\end{array}$ & 5 & 20 & 20 & 4.424 & 0.0389 \\
\hline WNFN & 2 & 2 & 20 & 4.076 & 0.8388 \\
\hline
\end{tabular}




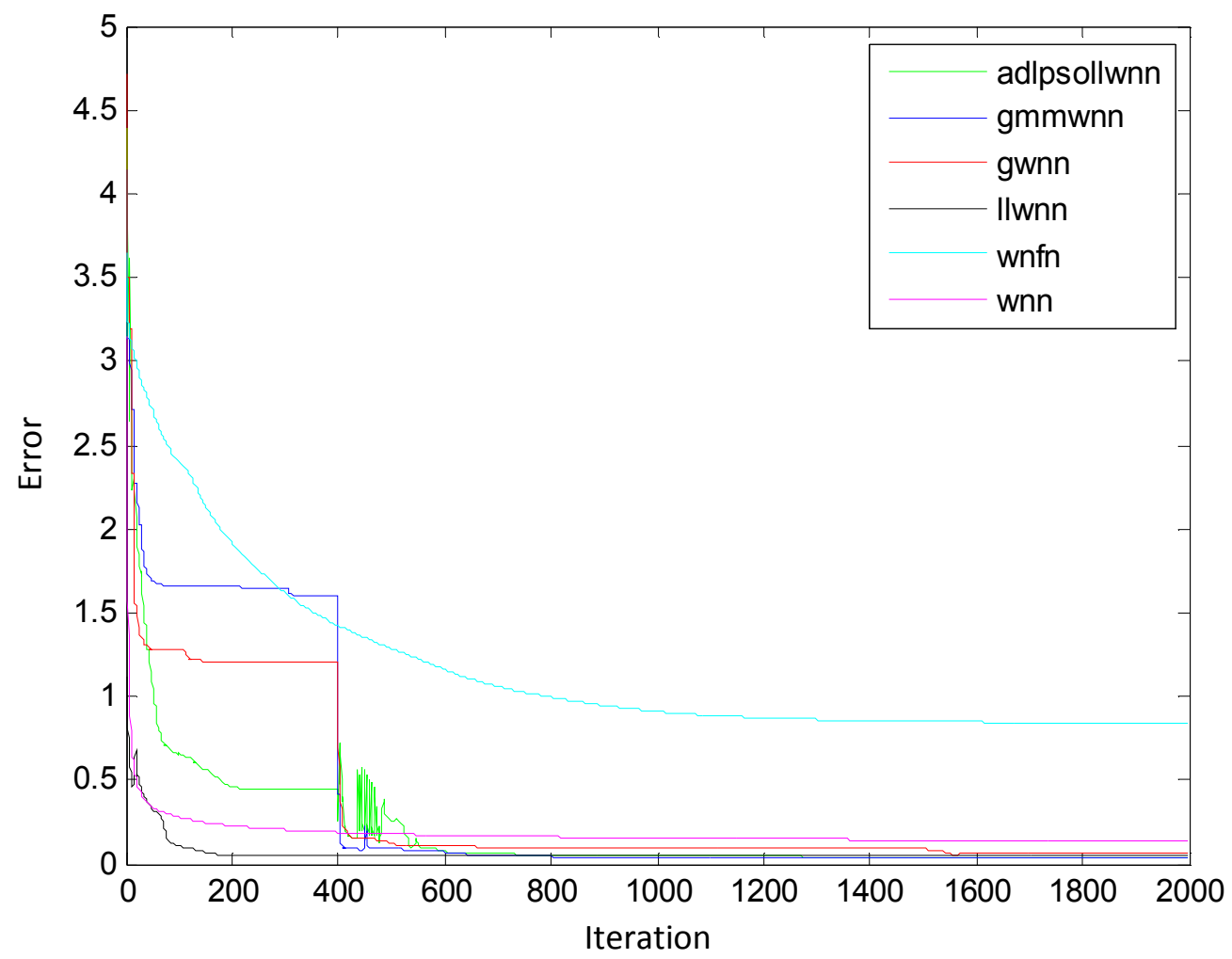

Figure 5.5 Objective function error for each model recorded for every iteration

\subsection{ECG Noise Reduction Comparison}

Electrocardiography or ECG is the recording of the electrical activity of the heart over time via skin electrodes. More often than not, contaminating noise appears as a byproduct while measurement is taken place. Formal introduction of ECG will be presented the next chapter. As the interest of this paper involves noise reduction in ECG, it is interesting to see WNNs performance aspect in this application. There are many types of contaminating noises in ECG, see chapter 6 . However for general comparison purposes, only white noise will be considered. White noise, unlike other ECG's noise, covers large frequencies band. Not only is it common in ECG, white noise can also be 
found in almost all application relating to signal processing. Therefore, if WNNs are able to perform well in the application of ECG, perhaps there's a potential for other noise reduction applications as well.

During this particular test, the structure size for each network is kept the same as in the previous section. Only the number of wavelons is increased to compensate for the higher complexity of ECG compared to previous tests. Moreover, due to the major difference in structural layout of the network, WNFN needs to be re-tested with different learning rates to accommodate for a higher number of rules and networks. Various combinations of rules and networks were used against several learning rates to find the optimal parameters. With an exception of WNFN, which has the learning rate of 0.5 , all others are kept at the same learning rate of 0.02 . See table 5.4 for specification of each network.

In testing, each network will be given 4,000 sample points for noisy ECG and 4,000 sample points for noise-free ECG during the training time. The noisy ECG is fed into the network's input and noise-free ECG is used for reference comparison at the output generating error necessary for back-propagation method in updating the networks parameters. Upon completion of training, a new set of 4,000 sampled noisy ECG, continued sampled data from training set, will be used for testing (see figure 5.6). Comparison between each WNN will be the length of training time as well as overall improvement in signal-to-noise ratio (SNR). 
Table 5.4 ECG Noise Reduction Results

\begin{tabular}{|c|c|c|c|c|c|}
\hline Method & $\begin{array}{c}\text { Number of } \\
\text { Wavelons }\end{array}$ & $\begin{array}{c}\text { Number of } \\
\text { Networks }\end{array}$ & $\begin{array}{c}\text { Number of } \\
\text { Parameters }\end{array}$ & $\begin{array}{c}\text { Training } \\
\text { Time (s) }\end{array}$ & $\begin{array}{c}\text { SNR* } \\
\text { Improved (dB) }\end{array}$ \\
\hline WNN & 100 & 1 & 301 & 1042 & 7.54 \\
\hline GWNN & 100 & 20 & 301 & 1513 & 8.00 \\
\hline GMM-WNN & 100 & 20 & 301 & 1580 & 7.55 \\
\hline LLWNN & 75 & 1 & 300 & 933 & 4.67 \\
\hline $\begin{array}{c}\text { ADLPSO- } \\
\text { LLWNN }\end{array}$ & 75 & 20 & 300 & 1361 & 4.10 \\
\hline WNFN & 10 & 10 & 320 & 3394 & 4.36 \\
\hline
\end{tabular}

*Method in which SNR are calculated for ECG related signal can be found in section 7.2.2.

The results collected from testing are shown in table 5.4 along with WNN's structure sizes, parameters, training time (in seconds), as well as SNR improvements over the original noisy ECG. For complete information on ECG related information such as sampling frequency and sources, see section 7.2. In figure 5.6, SNR of the noisy signal is found to be $17.7 \mathrm{~dB}$. WNNs will need to reduce the existing noise and improve overall SNR. Surprisingly, regular WNN, GWNN, and GMM-WNN are shown to have improved SNR over the other three. This is unexpected because of the result shown in previous tests where ADLPSO-LLWNN seemed show better results overall. There is, however, some convergence problems with GA and PSO. With the usage of either algorithm in combination with WNNs, roughly $30 \%$ of the tests would not converge. Meaning that, not only the noise would not be reduced, but that the network introduced additional noise to the signal. As a result, SNR declined. For the remaining $70 \%$, the networks were able to reduce the overall SNR by the average of $7.5 \mathrm{~dB}$ for GA (GWNN and GMM-WNN) and $4.0 \mathrm{~dB}$ for PSO (ADLPSO-LLWNN). This could be the result affected by how the GA and PSO settle down at the end of their trainings. After all, the results of both GA and PSO did depend somewhat from random numbers. In any case, the real cause of this 
effect is unknown. Further investigation is needed. However, because the point of this paper is not meant to focus on this specific topic, the author would like to leave the subject.

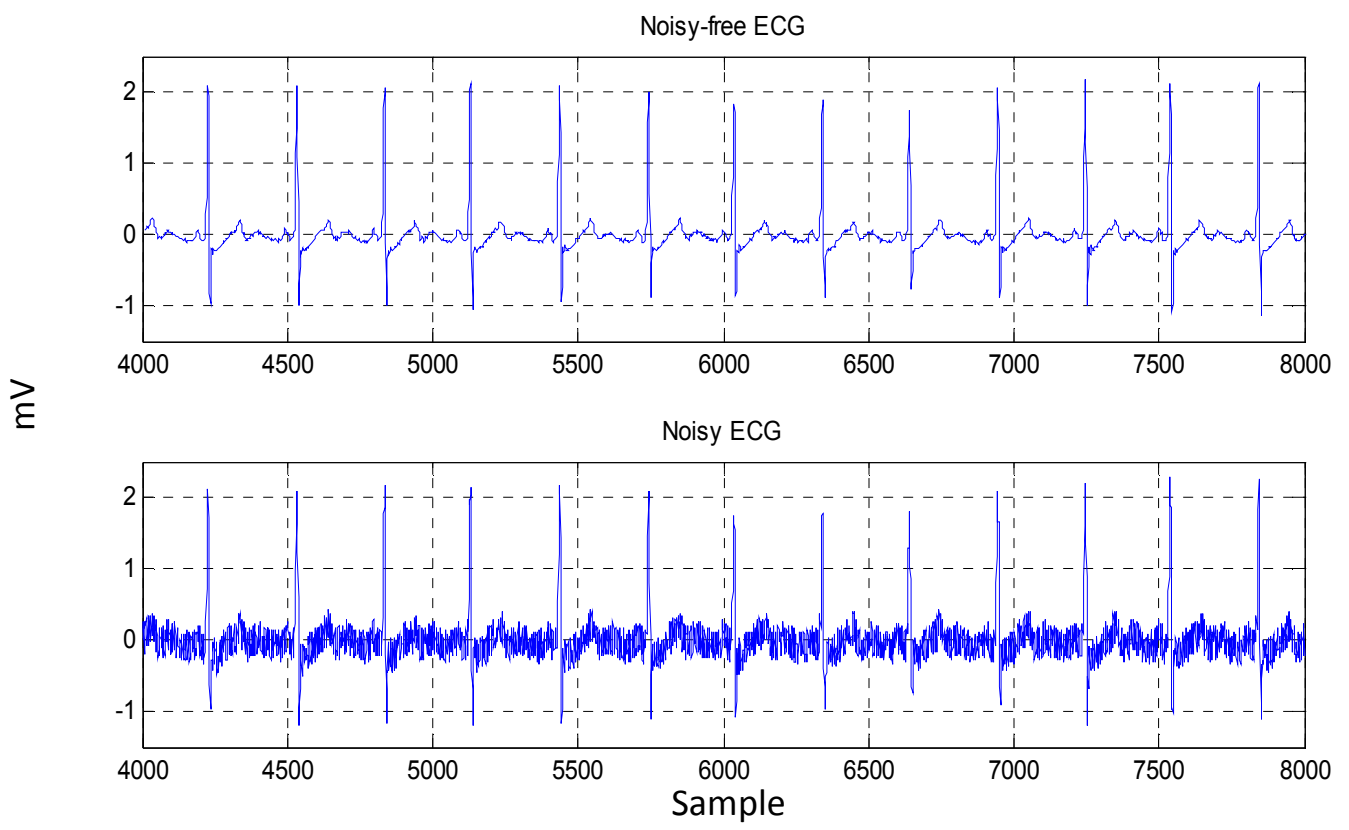

Figure 5.6 Original noise-free ECG (top) and Unfiltered noisy ECG signal (bottom)

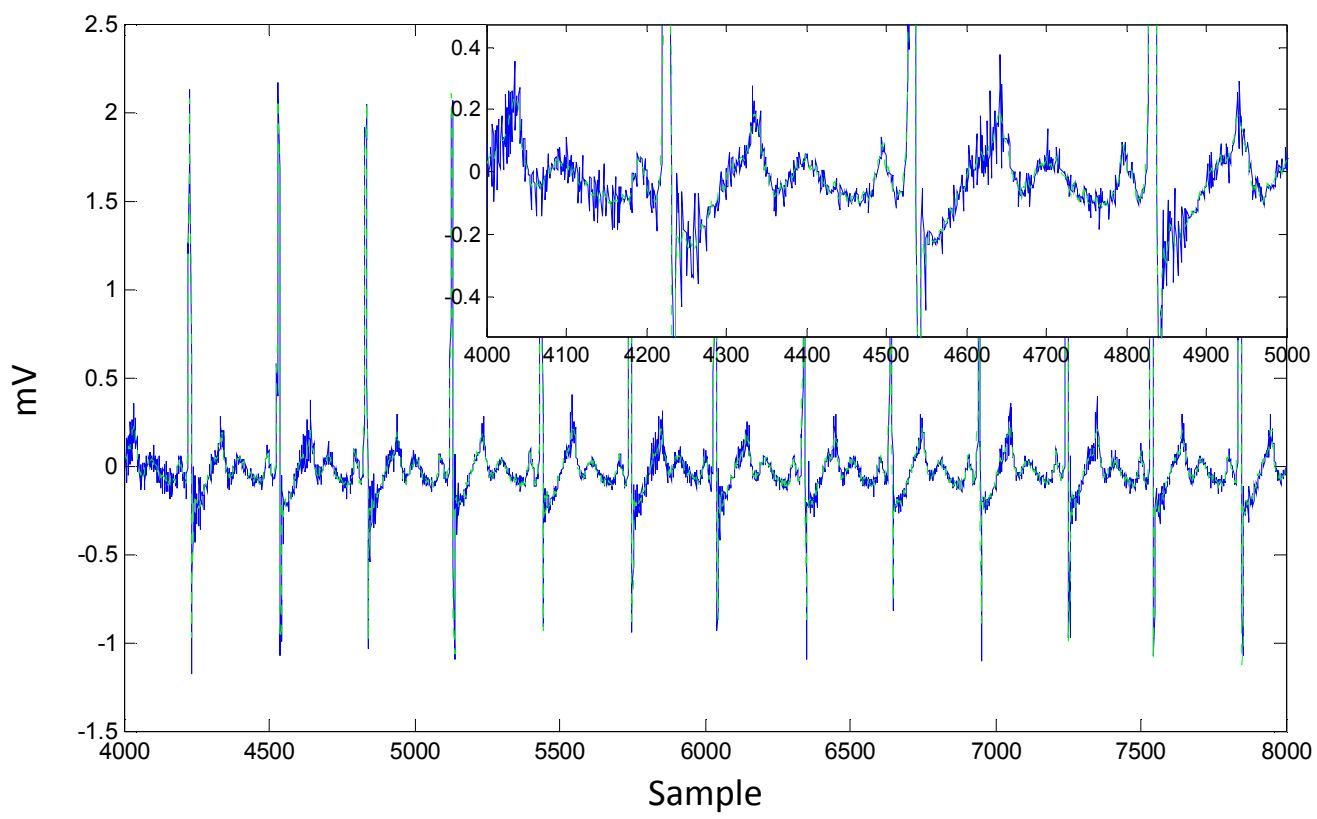

Figure 5.7 WNN result for ECG noise reduction 


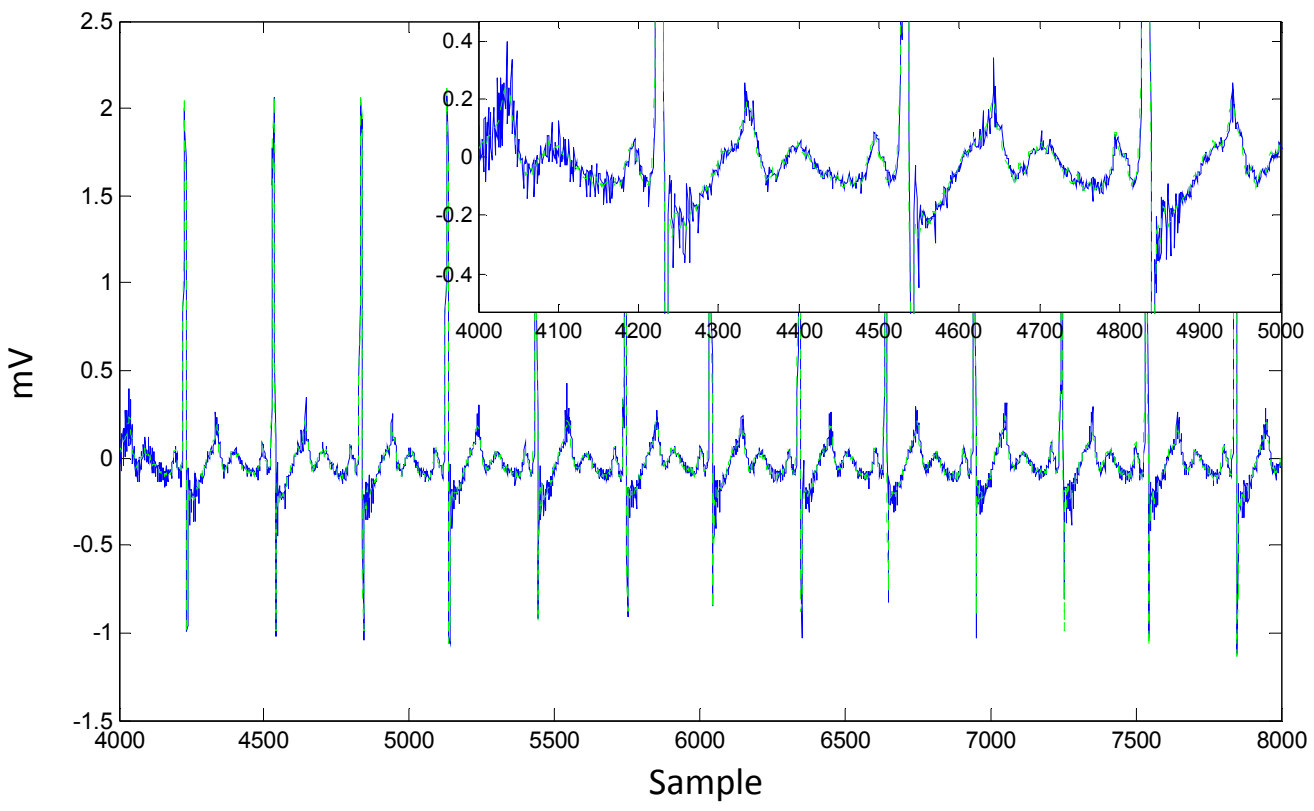

Figure 5.8 GWNN result for ECG noise reduction

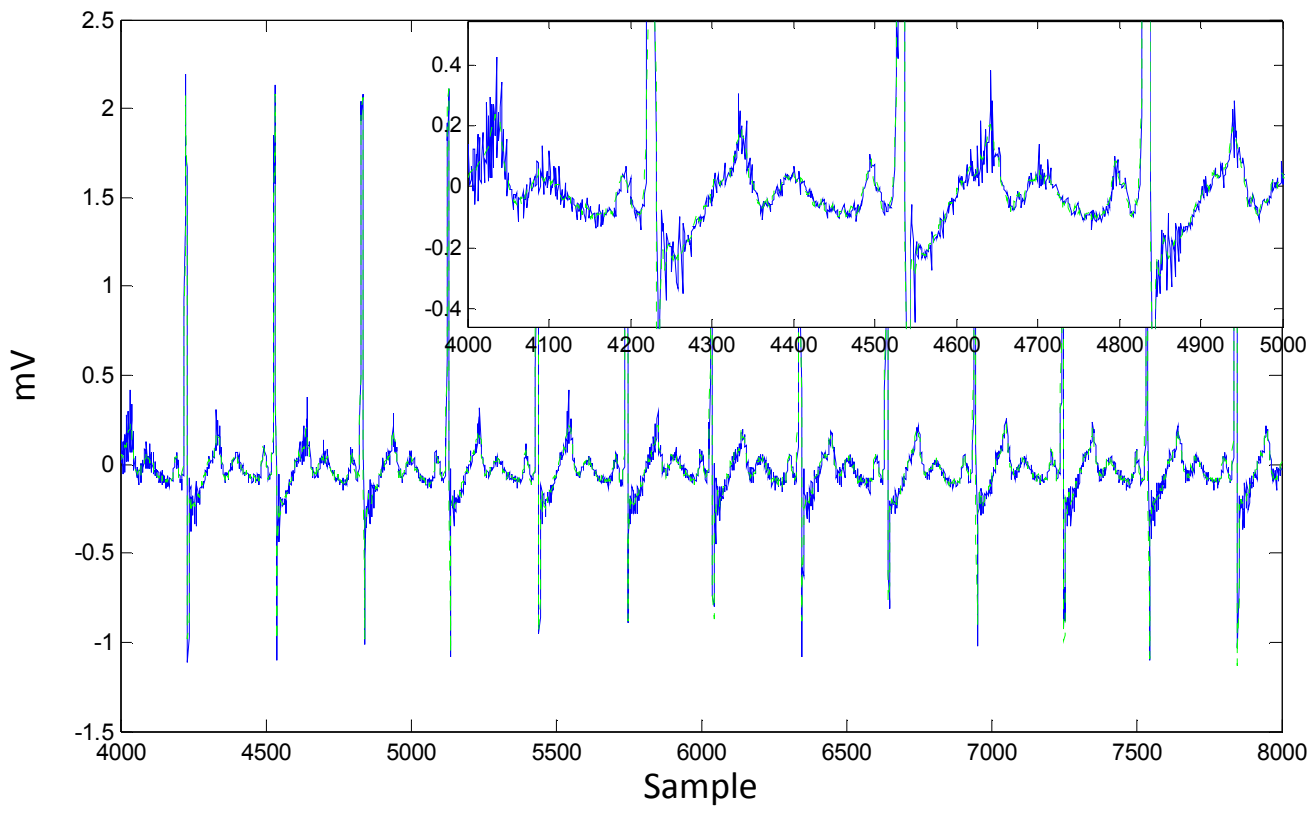

Figure 5.9 GMM-WNN result for ECG noise reduction 


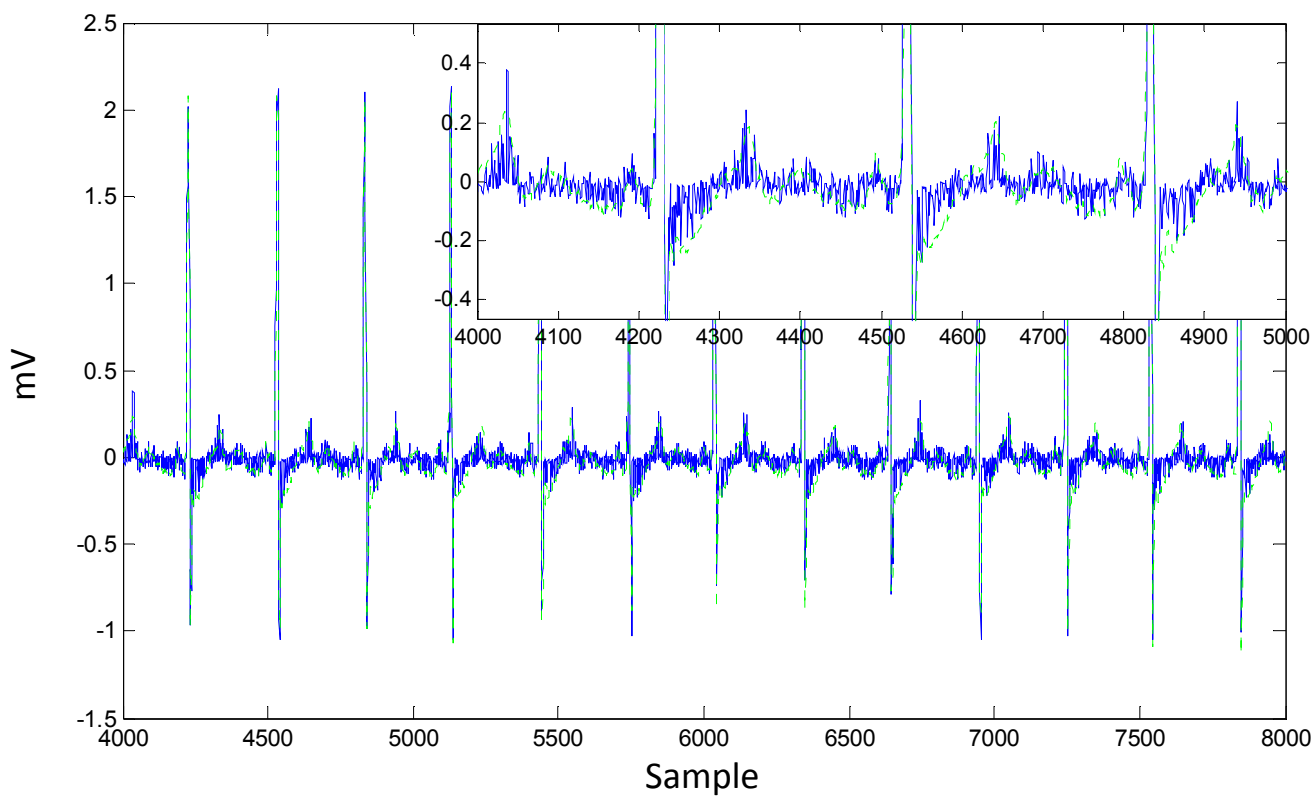

Figure 5.10 LLWNN result for ECG noise reduction

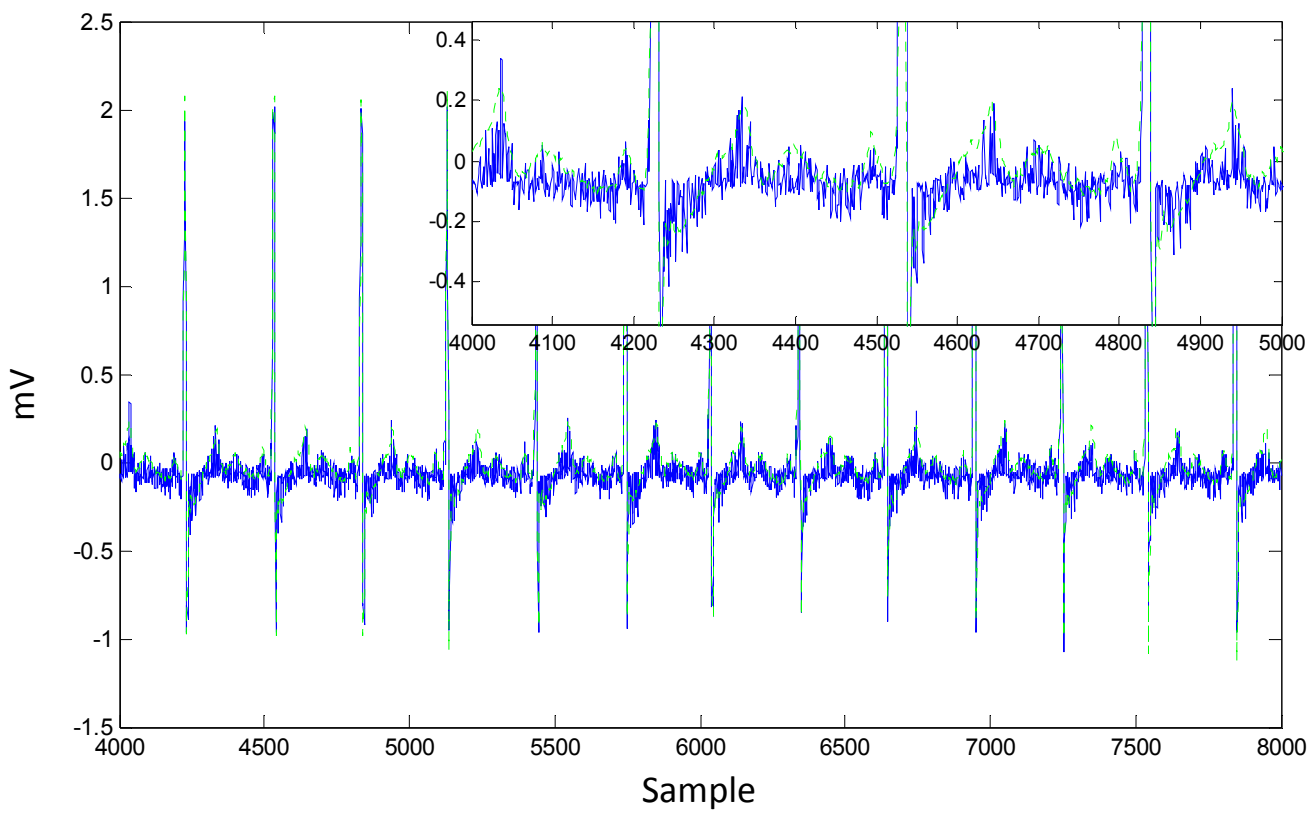

Figure 5.11 ADLPSO-WNN result for ECG noise reduction 


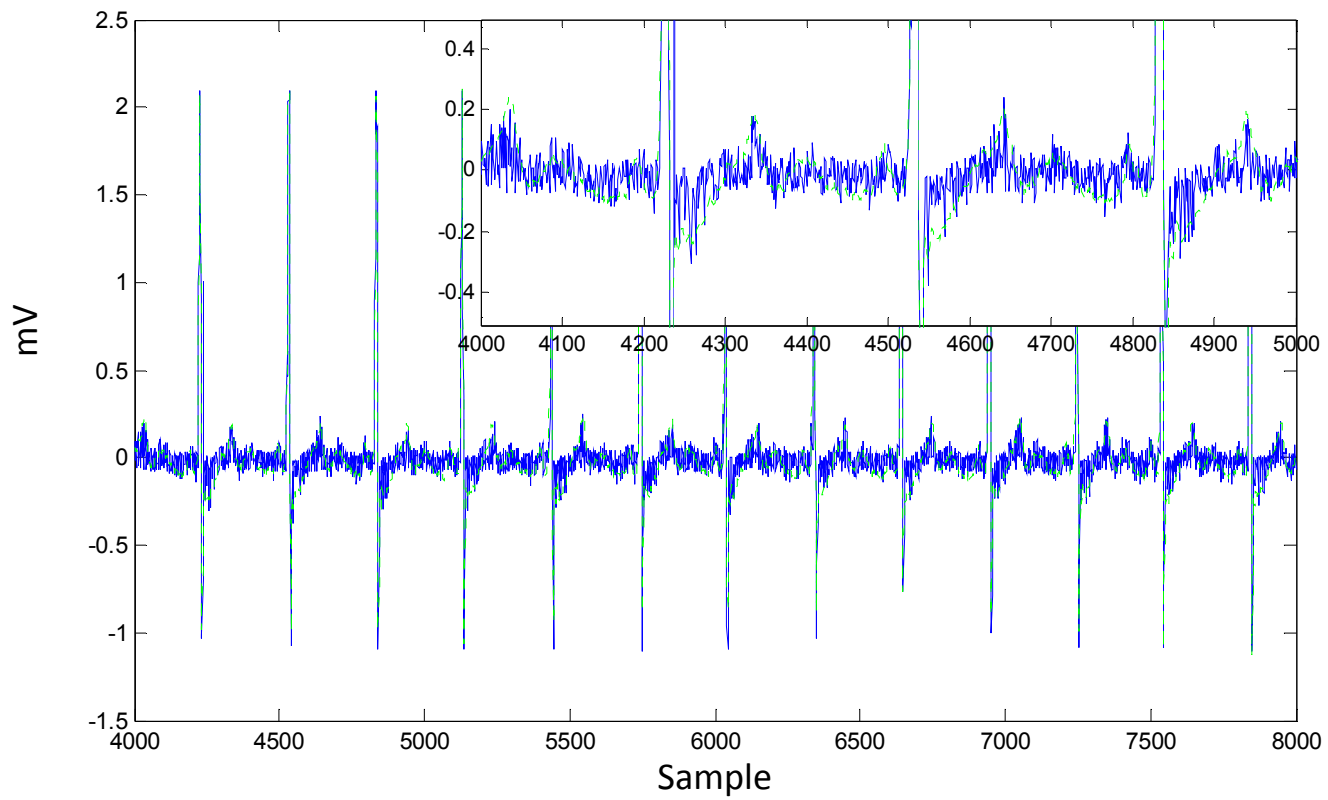

Figure 5.12 WNFN result for ECG noise reduction

\subsection{Conclusion}

In the attempts to improve upon the wavelet neural network, various designs and algorithms have been incorporated into the existing network. Some research has focused on modifying and adapting new structures into the network while others have tried to improve learning strategy and still, there are those that would combine two or more algorithms in hope of eliminating weaknesses in the original design.

The applications of system modeling and noise reduction were provided as comparison tests between each model. For modeling application, GMM-WNN and ADLPSO-LLWNN provided the two best results. This could be credited by the fact that GMM-WNN 
provides additional choices of activation function for different shape of input signals. As for ADLPSO-LLWNN, a diversification search, on top of an existing PSO, ensures a lesser chance of the network being trapped at a local minima.

For the next comparison in the application of noise reduction, WNN, GWNN, and GMMWNN are the three most improved networks while LLWNN, ADLPSO-LLWNN, and WNFN are the lower improved group. The only difference between these two groups is that the first group did not have their original WNN's structures altered in anyway. For the lower improved networks, all of them utilized local linear design. In additional, WNFN combines translation and dilation parameters into a single parameter. From these tests, GMM-WNN has good performance overall in both comparisons.

The investigations of WNNs and noise reduction have shown the effectiveness of the network to reduce white noise from a non-stationary a cardiac signal. Despite SNR improvement, the output signal still appeared noisy and unacceptable.

In the actual situation, there are several more interferences beside white noise. Some of them are much more difficult to remove than the white noise presented here. In chapter 7, another more suitable approach for noise reduction involving Wavelet and Neural Networks will be presented. 


\section{Chapter 6: ECG and Noises Artifact in ECG}

Biomedical signals like electrocardiogram (ECG) are often contaminated by noise sources such as power line interference and disturbances due to movement of the recording electrodes. In addition, biomedical signals often interfere with one another; signals due to muscle contractions often contaminate ECGs, for example.

In this following chapter, brief information regarding ECG will be introduced as well as various noise models and their characteristics associated with ECG. At the end of this chapter, a simple adaptive noise reduction algorithm will be presented and implemented.

\subsection{ECG signal and Its Component}

An electrocardiogram or ECG is a representation of the electrical activity of the heart muscle as it is recorded from the body surface. A basic wave form of ECG of one cardiac cycle is shown in figure 6.1 that consists of a P-wave, QRS-complex and a T-wave. The processes in which these waves are formed are summarized here in this section. 


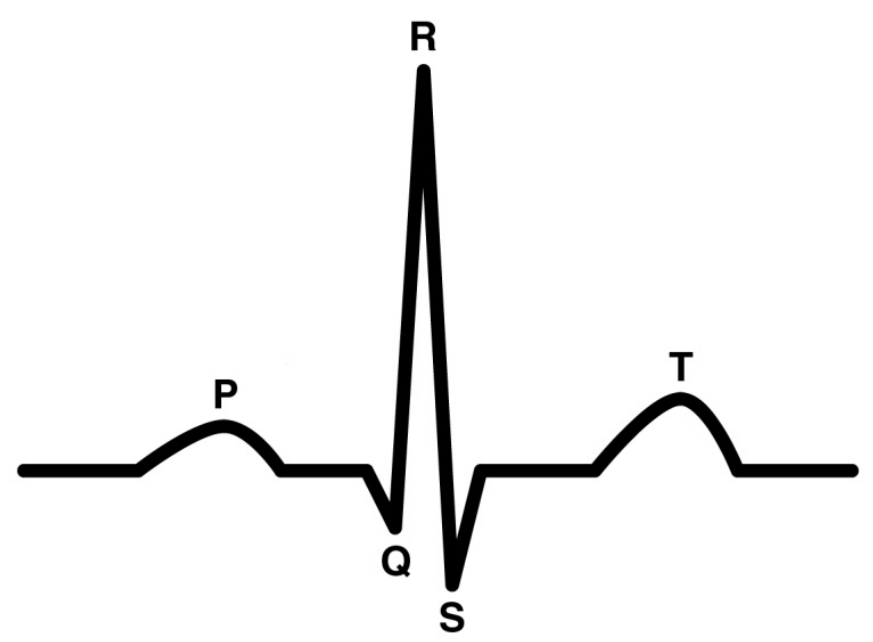

Figure 6.1 Basic ECG signal

The heart has its own built-in electrical system, called the conduction system [21], see figure 6.2. The conduction system sends electrical signals throughout the heart that determine the timing of the heartbeat and cause the heart to beat in a coordinated, rhythmic pattern as shown in figure 6.1. The electrical signals, or impulses, of the heart are generated by a clump of specialized tissue called the sinus node. Each time the sinus node generates a new electrical impulse, that impulse spreads out through the heart's upper chambers, called the right atrium and the left atrium. This electrical impulse, as it spreads across the two atria, stimulates them to contract, pumping blood into the right and left ventricle. The simultaneous contraction of the atria results in P-wave in figure 6.1. The electrical impulse then spreads to the atrioventricular (AV) node, which is another clump of specialized tissue located between the atria and the ventricles. The AV node momentarily slows down the spread of the electrical impulse, to allow the left and right atria to finish contracting. This causes a pause between the P-wave and Q-wave in figure 6.1. From the AV node, the impulse spreads into a system of specialized fibers 
called the "His bundle" and the right and left bundle branches. These fibers distribute the electrical impulse rapidly to all areas of the right and left ventricles, stimulating them to contract in a coordinated way. With this contraction, blood is pumped from the right ventricle to the lungs, and from the left ventricle throughout the body. This action produces the QRS-complex. Lastly, depolarization of the ventricular myocytes begins immediately after QRS and persists until the end of the T-wave.

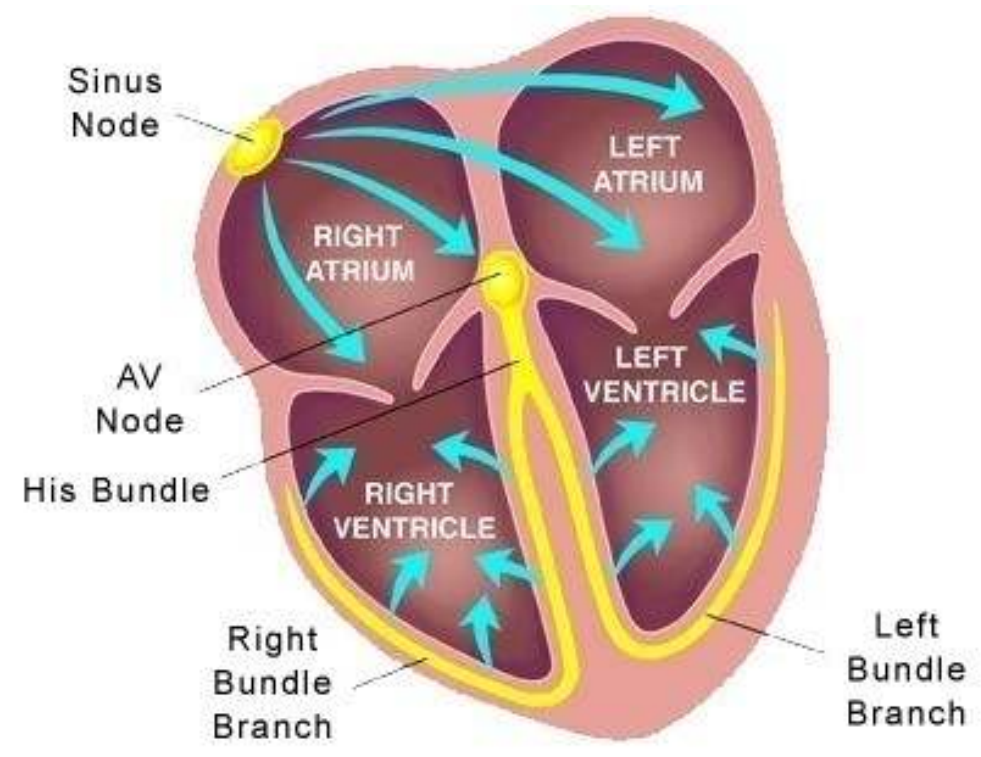

Figure 6.2 The conduction system of the heart. Image is taken from [22]

\subsection{Type of Artifacts}

Electrocardiographic (ECG) signals may be corrupted by various kinds of noise. Typical examples are power-line interference, electrode contact, motion artifacts, muscle contraction, baseline drift, instrumental noise, and electrosurgical noise [23]. 


\subsubsection{Power Line Interference}

Power line interference consists of $60 \mathrm{~Hz}$ pickup (in the U.S.) and harmonics that can be modeled as sinusoids and combination of sinusoids. Characteristic of power line noise include amplitude and frequency content of the signal. These Characteristics are generally consistent for a given measurement situation and, once set, will not change during a detector evaluation. Typical parameters are:

- Frequency content of $60 \mathrm{~Hz}$ (fundamental) with harmonics

- Amplitude up to $50 \%$ of peak-to-peak ECG amplitude

\subsubsection{Electrode Contact Noise}

Electrode contact noise is transient interference caused by the loss of contact between the electrode and the skin, which effectively disconnects the measurement system from the subject. The loss of contact can be permanent, or can be intermittent, as would be the case when a loose electrode is brought in and out of contact with the skin as a result of movements and vibration. This switching action at the measurement system input can result in large artifacts since the ECG signal is usually capacitively coupled to the system. With the amplifier input disconnected, $60 \mathrm{~Hz}$ interference may be significant.

Electrode contact noise can be modeled as a randomly occurring rapid baseline transition (step) that decays exponentially to the baseline value and has a superimposed $60 \mathrm{~Hz}$ component. This transition may occur only once or may rapidly occur several times in succession. Characteristics of this noise signal include the amplitude of the 
initial transition, the amplitude of the $60 \mathrm{~Hz}$ component, and the time constant of the decay. Typical parameters:

- Duration time $\sim 1$ second

- Amplitude of the maximum recorder output

- Frequency of $60 \mathrm{~Hz}$ Time constant about 1 second

\subsubsection{Motion Artifacts}

Motion artifacts are transient (but not step) baseline changes caused by variations in the electrode-skin impedance with electrode motion. As this impedance changes, the ECG amplifier sees a different source impedance, which forms a voltage divider with the amplifier input impedance. Therefore, the amplifier input voltage depends on the source impedance, which changes as the electrode position changes. The usual cause of motion artifacts will be assumed to be vibrations or movement of the subject. The shape of the baseline disturbance caused by motion artifacts can be assumed to be a biphasic signal resembling one cycle of a sine wave. The peak amplitude and duration of the artifact are variables. Typical parameters:

- Duration about $100-500$ milliseconds

- Amplitude- $500 \%$ of peak-to-peak ECG amplitude 


\subsubsection{Muscle Contractions (EMG)}

Muscle contractions cause artifactual millivolt-level potentials to be generated. The baseline electromyogram is usually in the microvolt range and is usually insignificant. The signals, resulting from muscle contraction, can be assumed to be transient bursts of zero-mean band-limited Gaussian noise. The variance of the distribution may be estimated from the variance and duration of the bursts. Typical parameters:

- Standard Deviation of $10 \%$ of peak-to-peak ECG amplitude

- Duration about 50 milliseconds

- Frequency Content-DC to $10,000 \mathrm{~Hz}$

\subsubsection{Baseline Drift with Respiration}

The drift of the baseline with respiration can be represented as a sinusoidal component at the frequency of respiration added to the ECG signal. The amplitude and frequency of the sinusoidal component should be variable. The amplitude of the ECG signal also varies by about 15 percent with respiration. The variation can be reproduced by amplitude modulation of the ECG by the sinusoidal component which is added to the baseline. Typical parameters:

- Amplitude variation of 15 percent of peak-to-peak (p-p) ECG amplitude

- Baseline variation of15 percent of p-p ECG amplitude variation at 0.15 to $0.3 \mathrm{~Hz}$ 


\subsubsection{Instrumentation Noise Generated by Electronic Devices}

Artifacts generated by electronic devices in the instrumentation system cannot be corrected. The input amplifier has saturated and no information about the ECG can reach the detector. In this case manual prevention and coercive action need to be undertaken.

\subsubsection{Electrosurgical Noise}

Electrosurgical noise completely destroys the ECG and can be represented by a large amplitude sinusoid with frequencies approximately between $100 \mathrm{kHz}$ and $1 \mathrm{MHz}$. Since the sampling rate of an ECG signal is 250 to $1000 \mathrm{~Hz}$, an aliased version of this signal would be added to the ECG signal. The amplitude, duration, and possibly the aliased frequency should be variable. Typical parameters:

- Amplitude about 200 percent of peak-to-peak ECG amplitude

- Frequency Content of Aliased $100 \mathrm{kHz}$ to $1 \mathrm{MHz}$ Duration for 1-10 seconds

Other kind of interfering noises include Gaussian noises: white noise and colored noises.

\subsection{Adaptive Noise Reduction}

Adaptive filtering techniques are shown to be useful in many biomedical applications including noise reduction. The basic idea behind adaptive filtering will be summarized 
here. The implementation of noise reduction will be presented using a Least-MeanSquare (LMS) algorithm as an example.

\subsubsection{Least Mean Square (LMS) Algorithm}

Let $d(n)$ be the contaminated signal $d(n)=s(n)+x(n)$ where $s(n)$ is clean signal and $x(n)$ is noise. Using an input noise $x(n)$ and an FIR filter $H(z)$, signal $d(n)$ can be estimated as shown below.

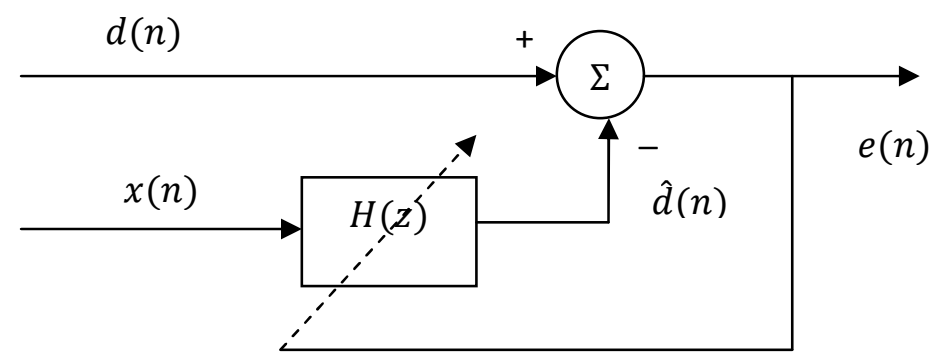

Figure 6.3 General LMS adaptive filter $H(z)$

There must be a correlation between $x(n)$ and $d(n)$. Because filter coefficients are adaptive to the signal and are updated for each $n$, this is a basic adaptive filter. The estimated signal $\hat{d}(n)$ is expressed by the following equation.

$$
\hat{d}(n)=\sum_{m=0}^{M} h_{m}(n) x(n-m)=\tilde{h}(n)^{T} \tilde{x}(n)
$$

where

$$
\tilde{h}(n)=\left[\begin{array}{c}
h_{0}(n) \\
h_{1}(n) \\
\vdots \\
h_{M-1}(n)
\end{array}\right] \text { and } \tilde{x}(n)=\left[\begin{array}{c}
x(n-d) \\
x(n-d-1) \\
\vdots \\
x(n-d-M)
\end{array}\right]
$$


Here, $d$ can be positive or negative. The error which is the difference between the signal and the estimated signal needs to be minimized by choosing the best filter coefficients. The error squared is to be minimized for each $n$ is given by

$$
J_{n}=e^{2}(n)=\left[d(n)-\tilde{h}(n)^{T} \tilde{x}(n)\right]^{2}
$$

Assume that a simplified error function with respect to the filter coefficients is shown in figure 6.4 .

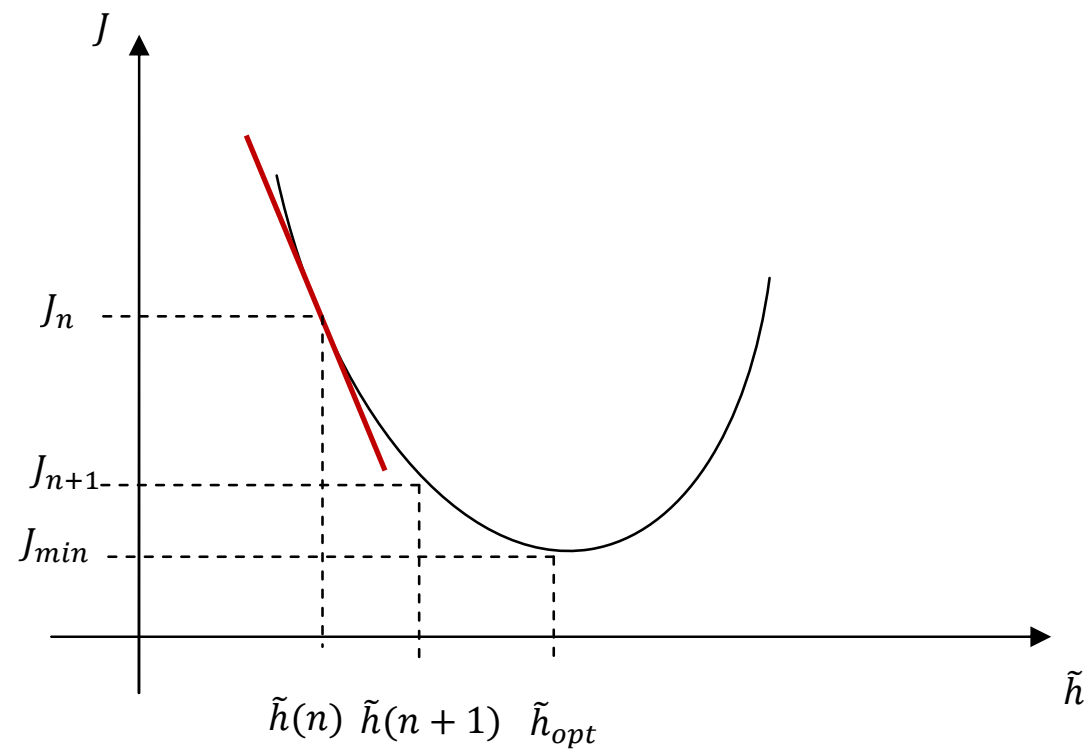

Figure 6.4 Simplified error function with respect to the filter coefficients

The slope or the first derivative of $J_{n}$ with respect to $\tilde{h}(n)$ is given by

$$
\frac{d J_{n}}{d \tilde{h}(n)}=-2 e(n) \tilde{x}(n)
$$

Let $\widetilde{h}_{\text {opt }}$ be the best or the optimum filter coefficients at which the squared error is minimized. If $\tilde{h}(n)$ is at the left-hand side of $\tilde{h}_{\text {opt }}$, the filter coefficients in the next step, $\tilde{h}(n+1)$, should move to the right (or positive direction). Note that the slope is 
negative in this case. On the other hand, if $\tilde{h}(n)$ is at the right-hand size of $\tilde{h}_{\text {opt }}$, the filter coefficients in the next step, $\tilde{h}(n+1)$, should move to the left (or negative direction). The slope is positive in this case. Thus, the filter coefficients in the next step should be equation (6.4).

$$
\tilde{h}(n+1)=\tilde{h}(n)+\mu\left(-\frac{d J_{n}}{d \tilde{h}(n)}\right)
$$

When the slope is negative, positive correction will be made. And when the slope is positive, negative correction will be made. The $\mu$ parameter is termed the adaptation parameter. If it is too large, there will be too much fluctuation in the filter coefficients. If it is too small, it will take a long time for the filter coefficients to converge. By plugging equation (6.3) into equation (6.4), an adaptive rule is obtained.

$$
\tilde{h}(n+1)=\tilde{h}(n)+2 \mu e(n) \tilde{x}(n)
$$

The summary of the adaptive algorithm is as follows:

1. Initialize $\tilde{h}(n)$ with any number. Random numbers are usually used.

2. Compute $e(n)=d(n)-\tilde{h}(n)^{T} \tilde{x}(n)$.

3. Update $\tilde{h}(n+1)=\tilde{h}(n)+2 \mu e(n) \tilde{x}(n)$.

4. Go to 2 and continue.

\subsection{Implementation and Result}

One simple but important application is in $60 \mathrm{~Hz}$ power line interference cancellation. A reference input signal, representing power line interference measuring from some part 
of the body, other than the ECG recording area, may be used to cancel the power line interference from the ECG. Because signal is measured at different locations of the body, phase shift characteristics of a reading power line signal is expected.

For this simulation, a $60 \mathrm{~Hz}$ power line signal generated by sinusoid is added to ECG signal. An adaptive filter is to be implemented using the same $60 \mathrm{~Hz}$ power line signal as reference input with added phase shift of $\pi / 2$ radian signifying time measurement delay. In addition, the amplitude is set to $50 \%$ of ECG's peak-to-peak. Adaptive filter is simulated over 2,000 sample points. Filtered signal is shown in figure 6.5. The filter was designed with the following specifications:

Table 6.1 LMS parameters

\begin{tabular}{|c|c|}
\hline $\mathbf{M}$ & $\boldsymbol{\mu}$ \\
\hline 5 & 0.02 \\
\hline
\end{tabular}
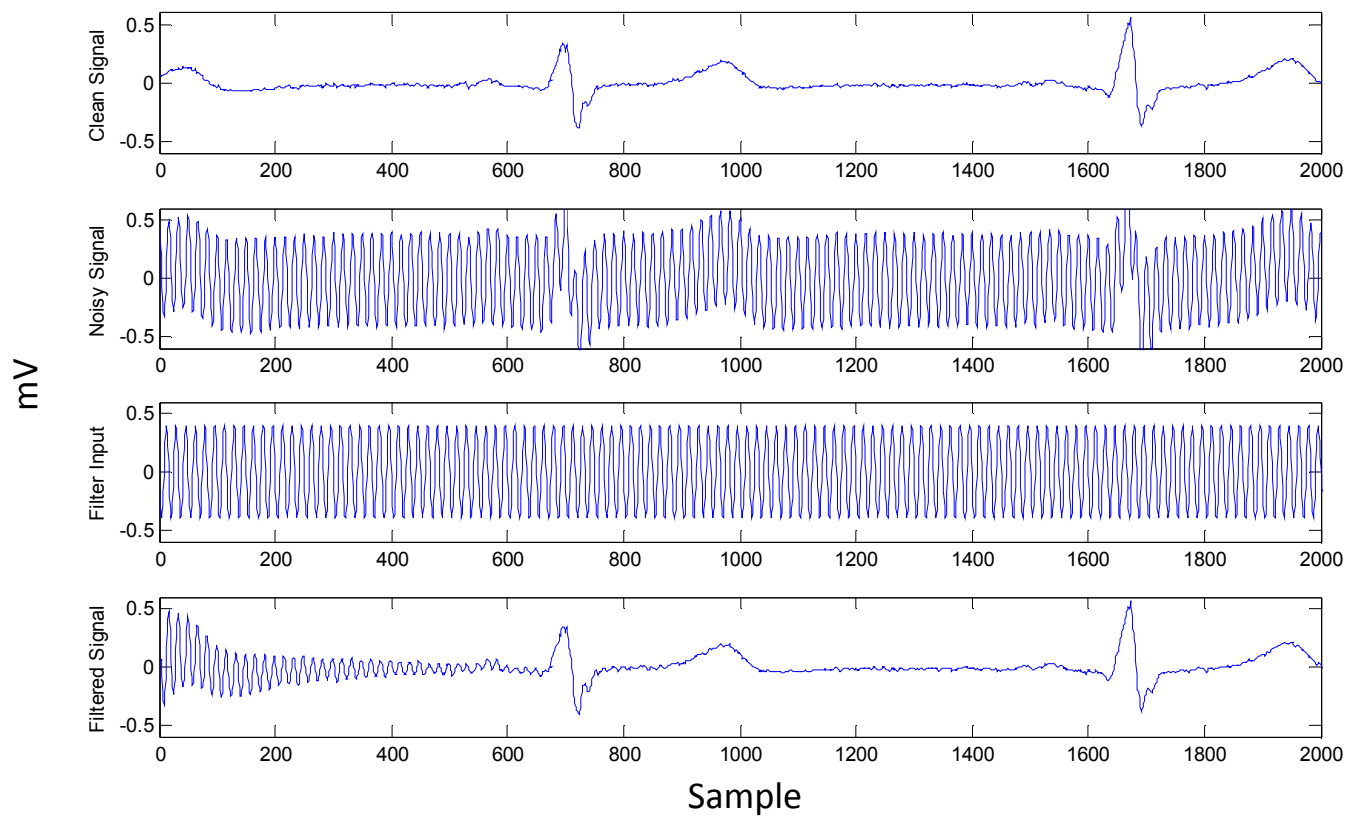

Figure 6.5 LMS result as ECG adaptive power-line noise cancellation 


\subsection{Conclusion}

This chapter has briefly introduced many of the commonly known noises associated with ECG signal as well as descriptions needed for reconstruction. Furthermore, a classical and simple noise reduction scheme implementing Least Mean Square (LMS) is also introduced.

LMS has shown to have a good noise cancellation result. The algorithm quickly converged and successfully removed power-line noise exposing the clean ECG signal as shown in figure 6.5. However, for LMS to work, both of the noise signals (measurement noise and noise contaminating signal) must be correlated to each other in some way. In addition, noise signals also need to be uncorrelated to the desired signal, ECG in this case. In a situation where the two noises are equal, LMS will be able to recover the original signal perfectly. But due to the nature of the error signal, the two noises will never be exactly the same. Noise, from where it is read, propagated through certain distance, and hence distorted in some way before it mixes with the ECG signal. In this case, LMS will never be able to fully recover the signal. To make matters worse, in some cases, the measurement noises may not be available at all making it impossible to implement LMS algorithm. In the next chapter, a proposed method of noise reduction utilizing the multi-resolution ability of wavelet technology and adaptability of neural networks will be introduced to overcome such problems. Noise reduction is implemented using wavelet decomposition and multi-layer neural network connecting in cascade formations. 


\section{Chapter 7: ECG Noise Reduction}

The results reported in chapter 4 look promising. A single-input-single-output (SISO) neural network with as little as six neurons was able to successfully train to tolerant noise on sinusoid waveform. For that given problem, the contaminated noise is a wideband while the desired signal is located at a single band. As an alternative and easier way to solve this problem, a band-pass-filter can be incorporated into the system and produce a cleaner input and hence avoiding the need to use neural networks for noise reduction for such purposes. In the application of ECG, however, the contaminated noises usually consists of frequency ranges that overlap with the ECG signal itself making it extremely difficult for filtering. Figure 7.1 provides frequency band spectrum of typical ECG signal taken from [30] of sampled rate $360 \mathrm{~Hz}$. As shown in the figures, these frequencies' range expand in the lower part of the spectrum to no higher than 70 $\mathrm{Hz}$ which also covers the range of the noises' frequencies shown in figure 7.2. The two frequencies plots displayed in figure 7.2 are electrode motion artifacts and muscle (EMG) artifacts taken from [25]. They are two of common ECG noises mentioned in chapter 6. But unlike other noises, these two are the most difficult noises to filter due to the spectrum overlap. Of these two, electrode motion artifact is generally considered the most troublesome, since it can mimic the appearance of ectopic beats and cannot be 
removed easily by simple filters, as can noise of other types [25]. Without the right technique to eliminate these types of noises, a doctor could misinterpret the signal. Therefore, he would not be unable to provide accurate diagnosis of the patients.

In this chapter, an adaptive method of neural network is proposed to remove ECG noises. One example of an adaptive filter was LMS algorithm for the $60 \mathrm{~Hz}$ power-line interference cancellation, shown in previous chapter, that utilize a reference signal representing power-line interference from some part of the body (other than the ECG recording area) may be used to cancel power-line interference from the ECG. But unlike the LMS algorithm, the proposed technique using neural networks does not require reference signals. This is due largely to cancellation of noises such as muscle (EMG) artifact that can differ from one part of the body to another due to the difference of motion movement between individual muscles. Rather, the technique is taking the advantage of the memorization capability of neural networks to learn the general signature of a patient pre-recorded noises-free ECG during the training phase. Wavelet decomposition is also utilized for frequency band separation making it easier for neural networks to recognize and interpret the signal. 


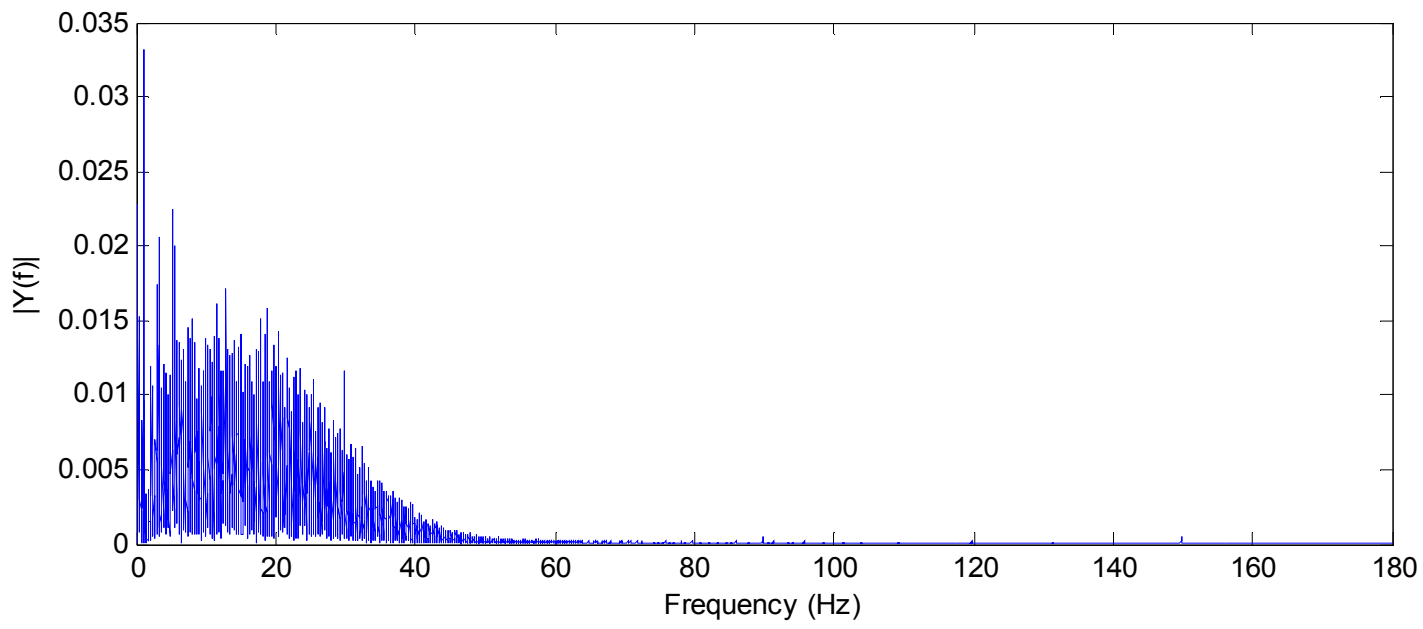

(a) Frequency spectrum of ECG record 115

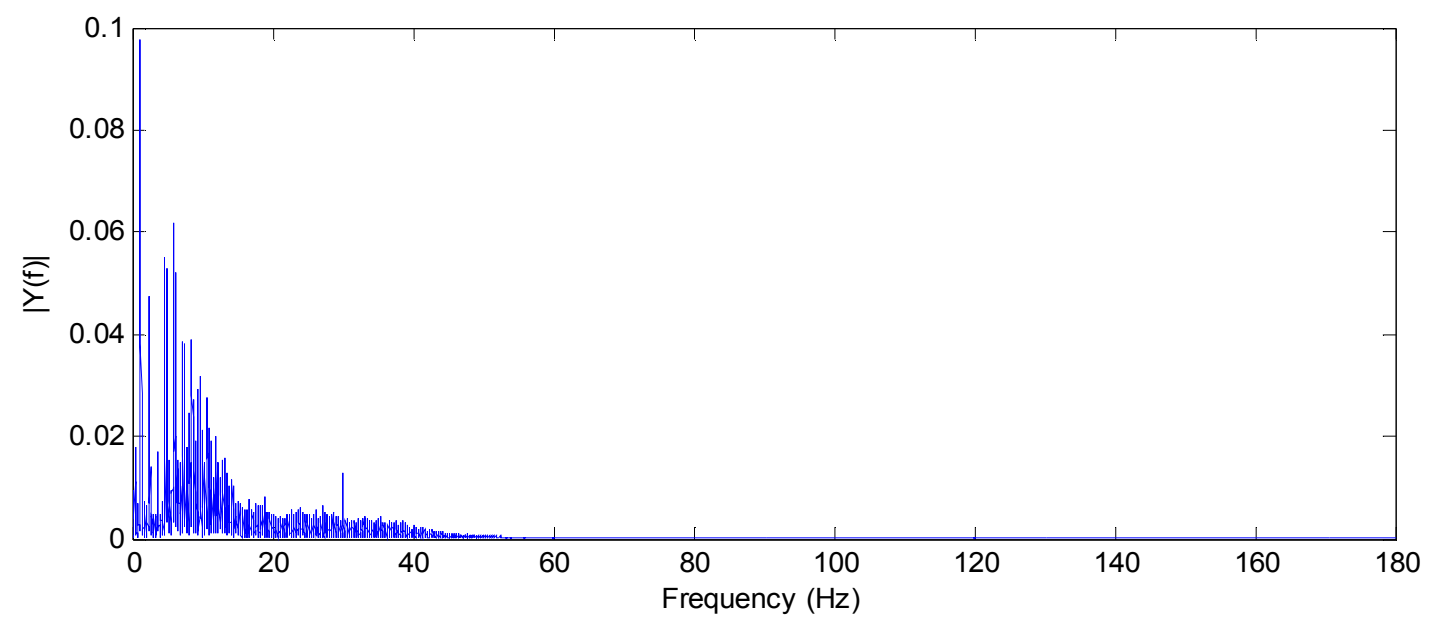

(b) Frequency spectrum of ECG record 118

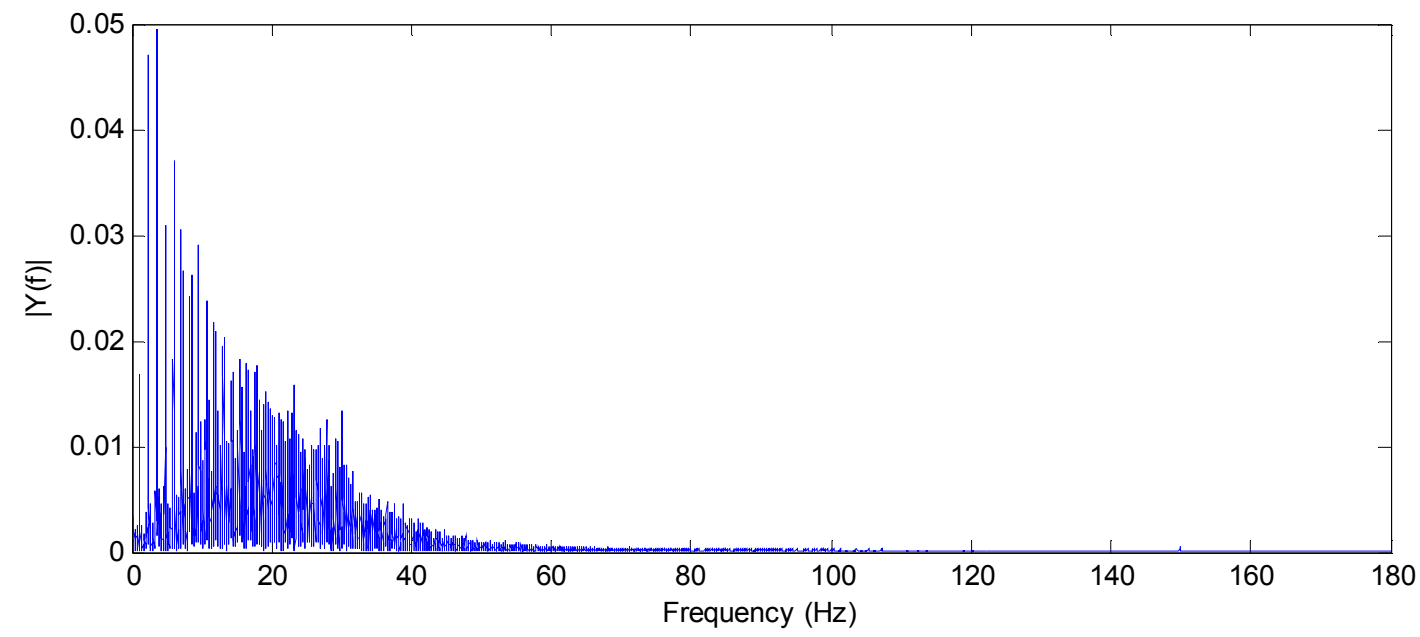

(c) Frequency spectrum of ECG record 220

Figure 7.1 Frequency spectrum analyses of typical ECG signals taken from [30] 


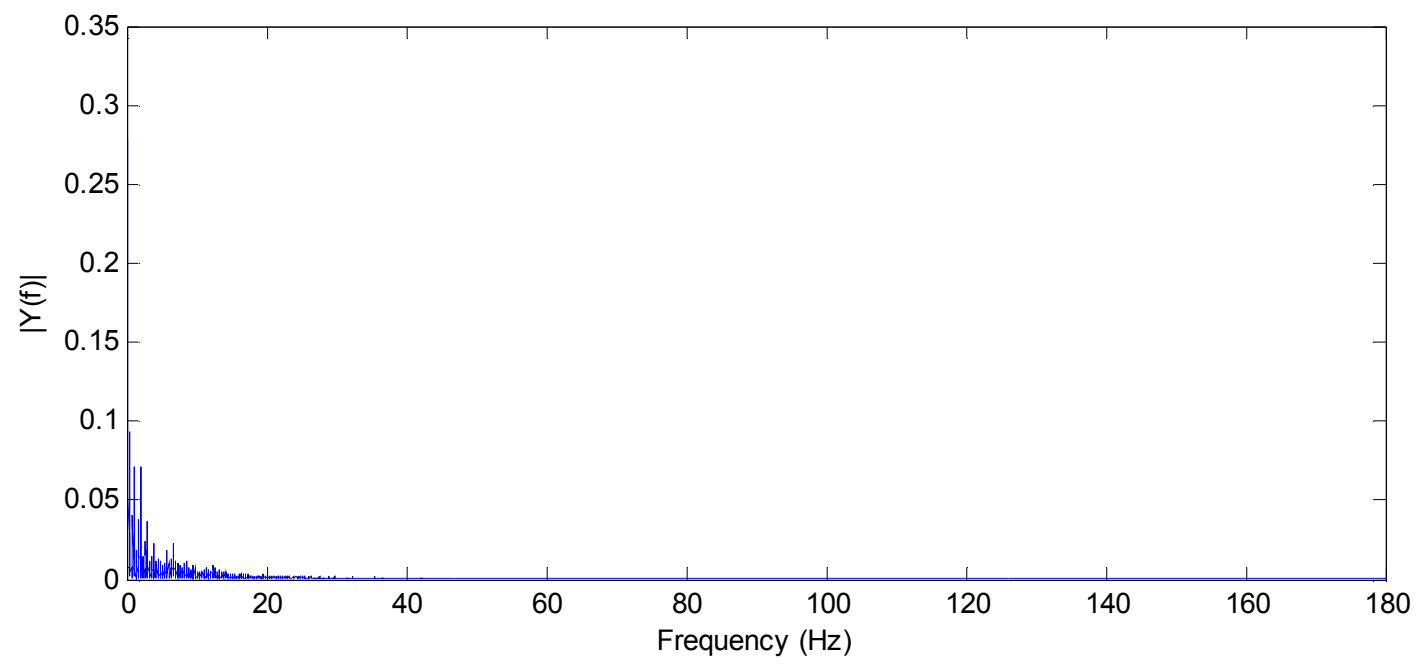

(a) Frequency spectrum of electrode movement

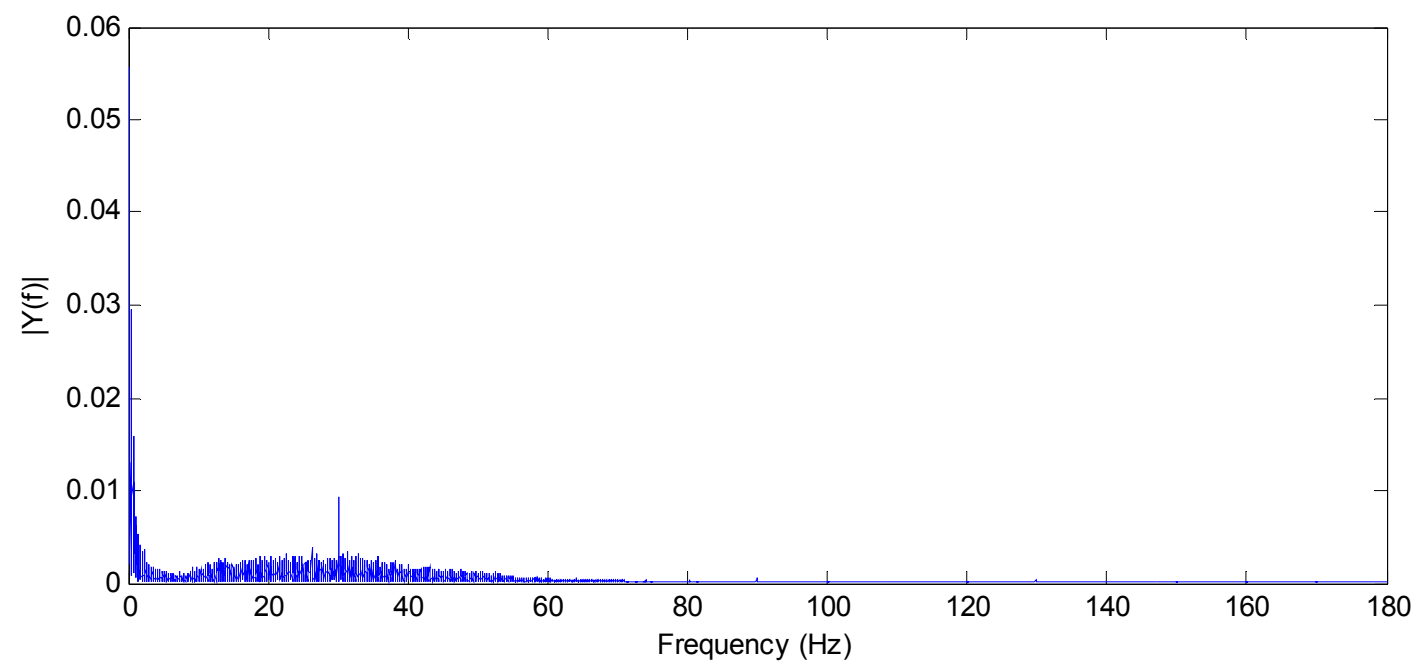

(b) Frequency spectrum of motion artifact

Figure 7.2 Frequency spectrum analyses of electrode movement and motion artifact taken from [25]

\subsection{Noise Reduction's Structure}

\subsubsection{Noise Reduction via Wavelet Decomposition and Neural Networks}


Figure 7.3 displays the overall scheme for noise reduction training and filter structure. The noisy ECG signal is decomposed by Discrete Wavelet Transform (DWT) into wavelet and scaling coefficients. Sub-band coefficient thresholding is then perform on these coefficients filtering only those that represent signals of interest and discard coefficients that do not represent ECG in anyway. More detail of the thresholding is explained in the next section. After the thresholding process, the remaining coefficients are those that truly represent and partially represent the ECG signal. Because noises among the coefficients still exist, a neural network is the last and final filtration process to remove the remaining noises mixture among the coefficients. In addition, neural network also performs Inverse Discrete Wavelet Transform (IDWT) on the signal. Thus, rendering the final filtered ECG signal. Note that normalization equation (7.1) and de-normalization equation (7.2) with $[-1,+1]$ limited interval are placed as pre and post-process of ECG signal.

$$
\begin{gathered}
\hat{s}(t)=2\left(\frac{s(t)-\min (X, Y)}{\max (X, Y)-\min (X, Y)}\right)-1 \\
s(t)=\frac{(\hat{s}(t)+1)(\max (X, Y)-\min (X, Y))}{2}+\min (X, Y)
\end{gathered}
$$

where $s(t)$ and $\hat{s}(t)$ are signal of interest and its normalized form, respectively. Variables $X$ and $Y$ are the complete training sets for the input signal and reference signal. This is because the hyperbolic (see table 2.1 ) is chosen as the activation function of neural network. Because of its characteristic, all signals must be normalized down to the $[-1,+1]$ range to accommodate for the activation function. The proposed neural 
network implemented in this chapter belong to class $N_{64,56,12,10}$. It is a simple multi-layer feed forward structure. This particular size of the proposed model is chosen based on numerous experimental runs using ECG record 220 (see figure 7.12). Note that input nodes of 64 are a restricted input size, resulting from DWT. In the next section, this input restriction will be explained clearly in detail. As for the hidden layers and output layer, these numbers are chosen based on experiments. Note that the output of the neural network is 10 . This does not imply that the network map its output 10 samples at the time during the actual test run. In fact, only one of those outputs is considered to be the output. The remaining outputs are only used during the learning process. An increase in output nodes allows the network to gain an extra references comparison to generate more errors which are utilized by back propagate algorithm to update weights in all the layers. Figure 7.4 displays error in relation between number of output nodes and signal-to-noise ratio (SNR). By inspection, the fitting trend-curve seems to flat out at about 10 outputs which indicate the minimum number of output nodes necessary for the application. Hence, output node of 10 is chosen. Once the training is completed, 9 out of 10 outputs will be discarded.

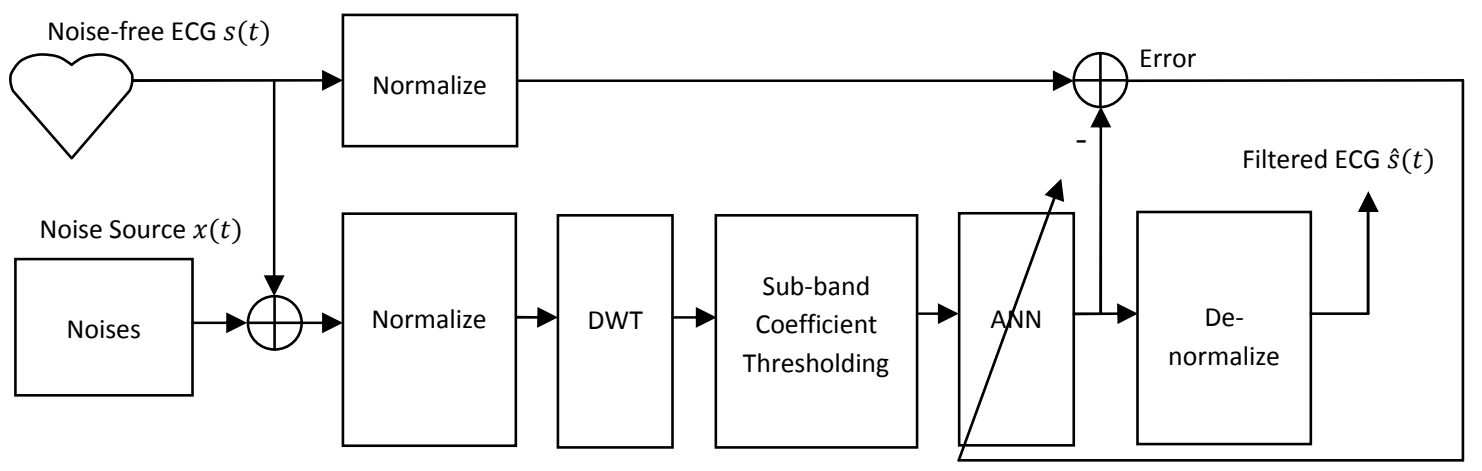

Figure 7.3 Overall adaptive noise reduction training scheme 


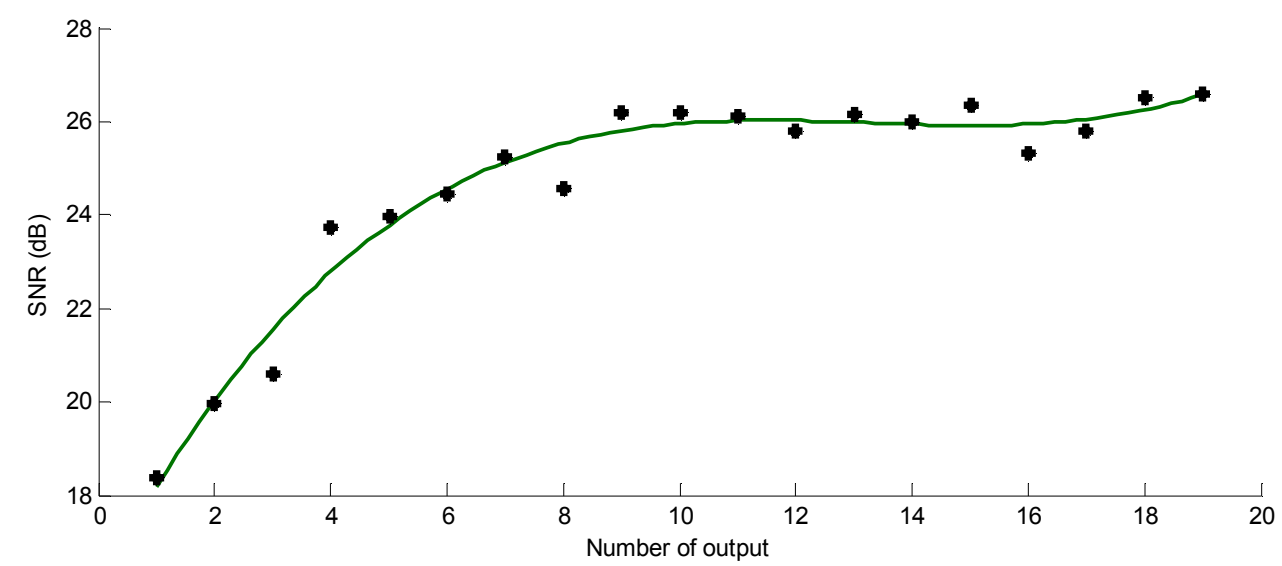

Figure 7.4 SNR gain comparing with number of output of neural network

\subsubsection{Discrete Wavelet Transform and Coefficient Thresholding}

The ECG signal during DWT pre-process after the normalization is put into cascade series form by delay units as shown in figure 7.5. The total length of this signal is chosen to be 256. This number is not chosen arbitrarily but based on the restriction of wavelet input which must be divisible by $2^{L}$ where $L$ is number of decomposing level. Once the coefficients are obtained, thresholding is performed to remove any irrelevant coefficients. The remainders are to be processed by a neural network.

There are several ways in which thresholding can be performed through DTW. The two most common methods are: soft and hard thresholding which usually requires a method of finding a threshold value at each transformed level either by finding a mean, standard deviation, or through a formula such as universal threshold (eq. 3.13) as demonstrated in chapter 3. These threshold methods can be done over selected scale and/or frequency, depending on the need of application. However, for noise reduction 
implemented in this paper, the unwanted coefficients are simply discarded - removed from the process.

The purpose of placing this sub-band coefficient threshoding serves two main purposes: first to remove unwanted frequency-noise bands which are mostly white noise and secondly is to help to reduce number of input nodes for neural network. As a result, there will be less unnecessary information for neural network to process. Hence, reduce the number of hidden nodes which further decrease the amount of time needed for training.

As mentioned in chapter 3 , the DWT process can be thought of as separating a signal into different frequency bands, using the series of low-pass-filter and high-pass-filter (see figure 3.6). If a signal is sampled at $360 \mathrm{~Hz}$, as it is the case in this paper, the first level of DWT will separate the signal into two pieces with the first half $0-180 \mathrm{~Hz}$ and the second $180-360 \mathrm{~Hz}$, roughly estimated. In the second level, the lower half is further split into another two portions: $0-90 \mathrm{~Hz}$ and $90-180 \mathrm{~Hz}$. In modern ECG monitors filtration for diagnostic mode, the range of interest is between $0.05 \mathrm{~Hz}$ at the lower end of spectrum which allows for ST segments to be recorded and 40,100, or $150 \mathrm{~Hz}$ in the higher end spectrum [32]. The choice for the frequency at the higher end depends on the patients' health and profile conditions as well as what type of diagnostic a doctor wants to capture. For instance, if a patient's heart beat is rapid and the QRT complex expands at a very short interval, ECG frequency spectrum may need to cover as high as $100 \mathrm{~Hz}$. Also, the characteristic of some heart related diseases may only exist at higher frequencies. 
By allowing higher cut-off frequencies to cover larger bands, a doctor will be able to diagnose a patient's condition. In this paper, the tested ECG have characteristics shown in figure 7.1; they cover at a maximum of around $80 \mathrm{~Hz}$. Allowing the convenience of current sampled frequency of $360 \mathrm{~Hz}$ and the restrictive nature of DWT, the frequency range for noise reduction implemented in this paper is chosen to be $0-90 \mathrm{~Hz}$ (frequency range of scaling coefficient after the second level). In other words, if 256 sampled points are to be transformed through DWT, only 64 coefficients are to be processed by a neural network. Again, because the analysis of the three patients' heart rate from figure 7.1 is shown to be $70 \mathrm{~Hz}$ or less, anything higher are noises and simply can be discarded. By doing so, not only are some of the unwanted noises removed, but also a lesser amount of input nodes is needed for a neural network.

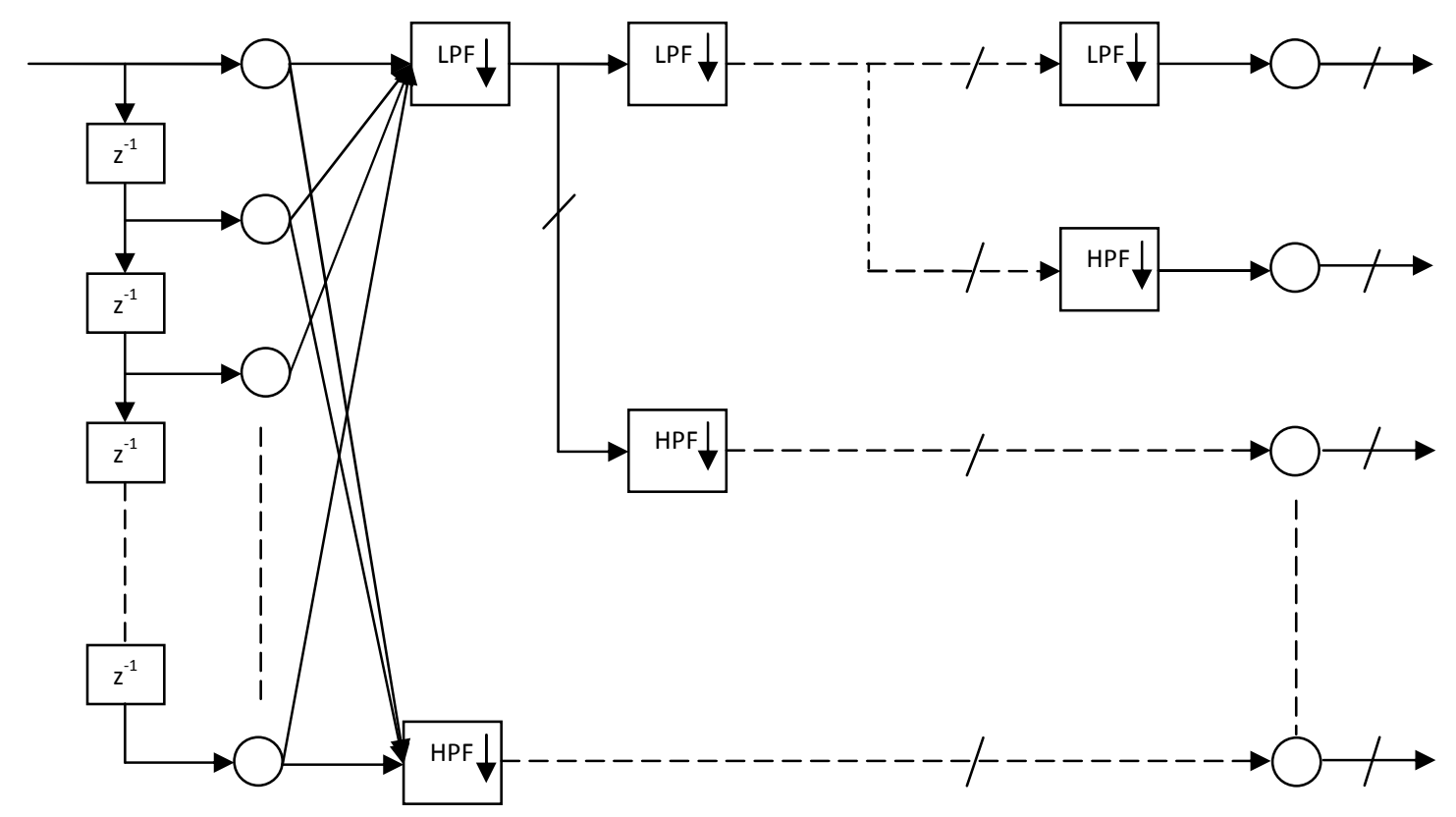

Figure 7.5 Wavelet Decomposition and coefficients thresholding 
The DWT resolution is dictated by the level of the transformation. However, for a discrete signal, the number of levels a signal is allowed to transform is limited by the number of input samples. In other words, a signal length of 8 can only be split in half at the most 3 times. This translates to a maximum number of levels DWT can perform on this signal. Hence, a signal of 256 in length can be transformed 8 levels at maximum. This is a number in which will be implemented in DWT at the end of this chapter.

\subsection{Data}

\subsubsection{Noises selection}

A noise reduction test is performed on three different patients. In this paper, only five types of noises are considered for noise reduction application. They are baseline wander, electrode motion artifact, muscle (EMG) artifact, Gaussian white noise, and $60 \mathrm{~Hz}$ power-line. For the remaining noises: electrosurgical noise, noise from electronic devices, and instrumentation noise will be not included here because these noises behave similarly to the random model in EMG. Using the same reasoning, motion artifact is much like the baseline drift in respiration so it is also not included [23].

All the ECG signals are taken from [30]. The three noises: baseline wander, electrode motion artifact, and muscle (EMG) artifact are taken from [25]. Every file in the database consists of 2 lead recordings sampled at $360 \mathrm{~Hz}$ with 11 bits per sample of 
resolution. The last two noises: Gaussian white noise and power-line interference are generated using Matlab.

The signal used under training is in the first 4,000 samples from a patient with the corruption added to the signal. After the training, the next following portion of 4,000 samples, both from ECG and noises, will be used for testing. In the first set of test, individual noise will be done separately. Next, the simulation will take a composite of all noises and add that to the ECG signals. Again, the network will be allowed to have the first 4,000 samples as training set. When training is completed, a portion of 4,000 samples (excluding any overlap from the first 4,000 sampled used during training) will be selected from the database to be used as testing data.

The performance evaluation discard results from training and only considers the results from testing.

\subsubsection{Calculating SNR}

The methodology, in which signal-to-noise ratios (SNR) is calculated, is based on reference [33]. SNR is commonly expressed in decibels (dB):

$$
S N R=10 \log \left(\frac{S}{N}\right)
$$

where $S$ is the power of the signal and $N$ is the power of the noise. However, unlike the typical calculation, $S$ and $N$ cannot be taken and calculated based on mean squared amplitude due to the complications described in [33]. In view of these issues, [33] 
defines $S$ as a function of the QRS amplitude, and $N$ as a frequency-weighted noise power measurement.

Power signal $S$ can be determined by locating the individual QRS complexes then expanding them into windows of 100 ms or 18 samples points on both side of the QRS's center given a sampled frequency of $360 \mathrm{~Hz}$ to measure the peak-to-peak amplitude. The largest $5 \%$ and the smallest $5 \%$ of the measurements are discarded. The estimated peak-to-peak of QRS is measured as the mean amplitude of the remaining $90 \%$. By taking the squares of this estimated peak-to-peak amplitude and dividing the result by 8 (correct for sinusoids and is close enough for this purpose), the QRS power, or estimated $S$, is obtained.

To determine $S$, noises' windows are also measured in the similar fashion as described above using the QRS complexes as a center reference. For each of those windows, the mean amplitude and the root mean squared difference between the signal and this mean amplitude is calculated. Similarly to the calculation of $S$, the largest $5 \%$ and the smallest $5 \%$ are discarded and estimated RMS noise amplitude as the mean of the remaining $90 \% . N$ is the square of this estimate.

The calculations of $S$ and $N$ are performed separately for each pair of the clean and noise signals. 


\subsection{Simulation Result and Discussion}

The effectiveness of the proposed method is demonstrated in this section. First, the network is trained to remove one type of noise at a time. By doing so, one can examine the results closely to the reaction of the individual noise whether or not the network performance meets the acceptable margin. Next, the simulation will combine all the noises as it would in a real ECG diagnosis situation and have the network train to remove those noises. To further test the effectiveness, the network is trained with different patients' ECG as well. The test also measures the performance and consistency of how well the network is able to learn by repeatedly training it with the same training data set and also testing it with the same testing data set. The only different is the initialization of the parameters. Finally, the experiment runs several comparisons with alternative selections of network models and shows the actual improvement of the proposed method over traditional method.

\subsubsection{Removal of single noise}

The test results for the removal of an individual noise are shown in table 7.1 and the graphical results are shown in figure 7.6-10. As one would expect, a power-line is the easiest to remove due to its stationary characteristic where the periodic cycle can be easily predicted. Next are baseline wander and Gaussian white noise. These two are also easy to remove despite the non-stationary characteristic of baseline drift. Both baseline and Gaussian noise are located at the very low-end and wide-band frequency spectrum, respectively. The upper-end of Gaussian white noise frequencies were already removed 
through the threshold process of DWT. The output of 64 coefficients from DWT to a neural network contains a scaling coefficient which represents the lowest end of a spectrum of $1.4 \mathrm{~Hz}$ and lower. This coefficient contains both noise as well as an ECG signal. Due to maximum level restriction of DWT, this scalar cannot be split any further. Thus, a neural network performed most of the filtering to remove baseline noise.

Both Electrode motion and EMG were expected to be the two most difficult to remove due to reasons explained earlier in this chapter. The improved SNR for Electrode motion is the lowest compared with the rest; nevertheless, results still looks acceptable. In figure 7.7, some of the high amplitude spits from the noise are still visible after the filtration. Most noticeable is the one located at the beginning of the signal around a sample point of 4,300 . High spits, like this one, often confuse a neural network tricking it to think that it is one of the ECG signatures as its morphology often resembles that of the $\mathrm{P}, \mathrm{QRS}$, or T waves.

Table 7.1 Individual Noise Removal Summary

\begin{tabular}{|l|r|r|r|}
\hline \multicolumn{1}{|c|}{ Noise } & $\begin{array}{c}\text { Pre-filter } \\
\text { SNR (dB) }\end{array}$ & $\begin{array}{c}\text { Post-filter } \\
\text { SNR (dB) }\end{array}$ & $\begin{array}{c}\text { Improved } \\
\text { SNR (dB) }\end{array}$ \\
\hline Baseline Wander & 19.18 & 30.74 & 11.56 \\
\hline Electrode Motion Artifact & 15.65 & 25.29 & 9.64 \\
\hline Muscle (EMG) Artifact & 22.90 & 28.09 & 5.19 \\
\hline 60 Hz Power-line & 10.95 & 33.31 & 22.36 \\
\hline Gaussian White Noise & 17.69 & 29.49 & 11.80 \\
\hline \multicolumn{3}{|c|}{ Average Improvement of SNR } & 12.11 \\
\hline
\end{tabular}

Note: SNR for ECG signal is calculated according to [33]. 

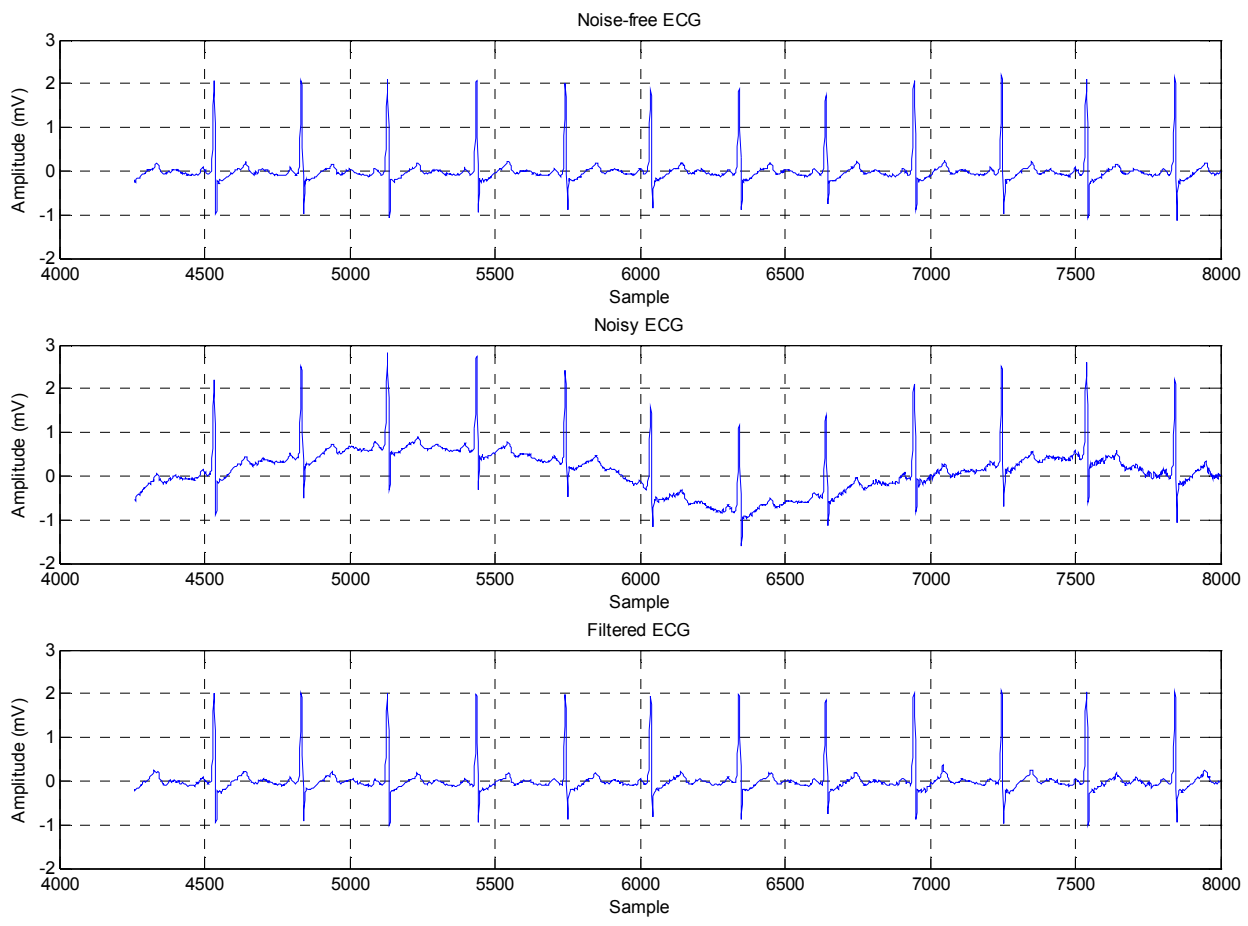

Figure 7.6 Baseline wander removal
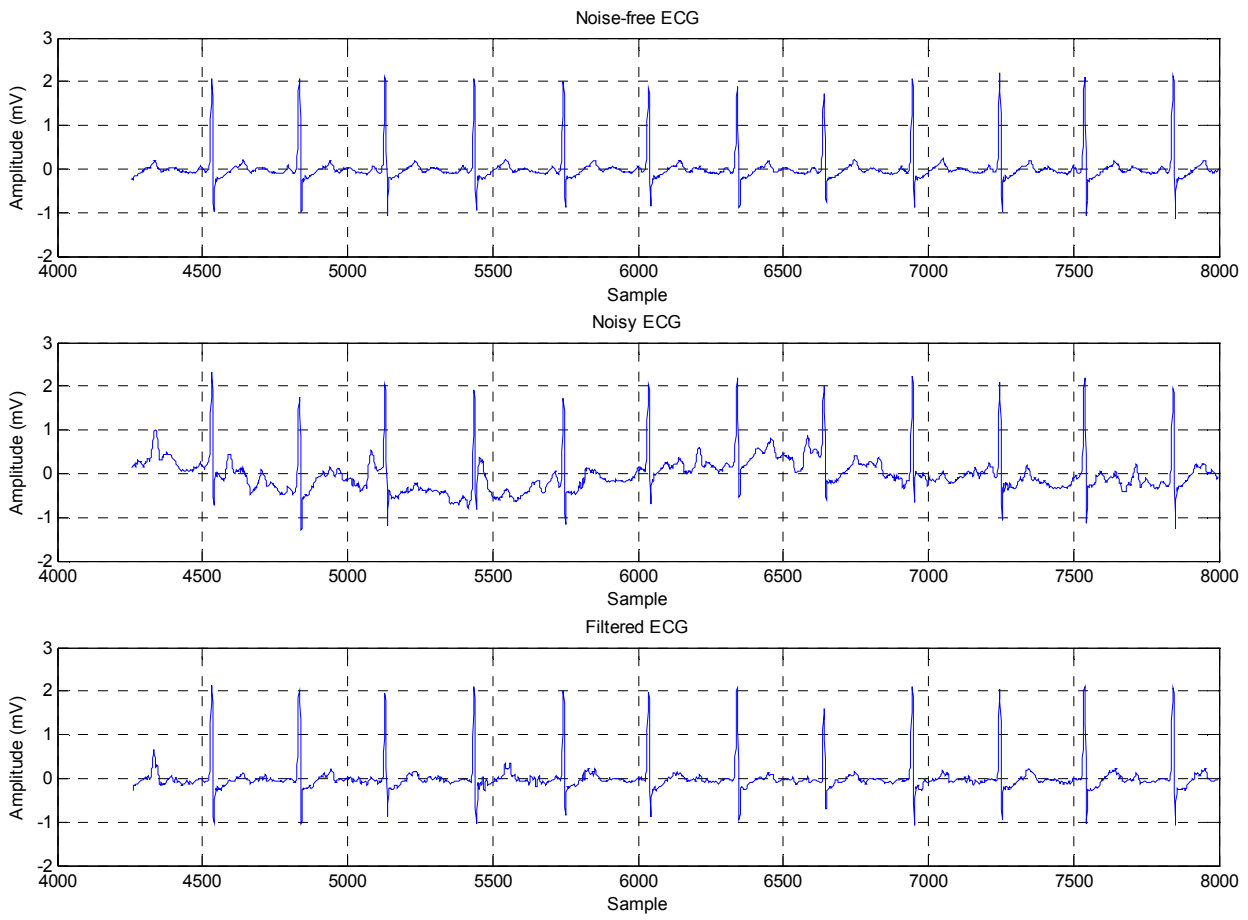

Figure 7.7 Electrode motion artifact removal 

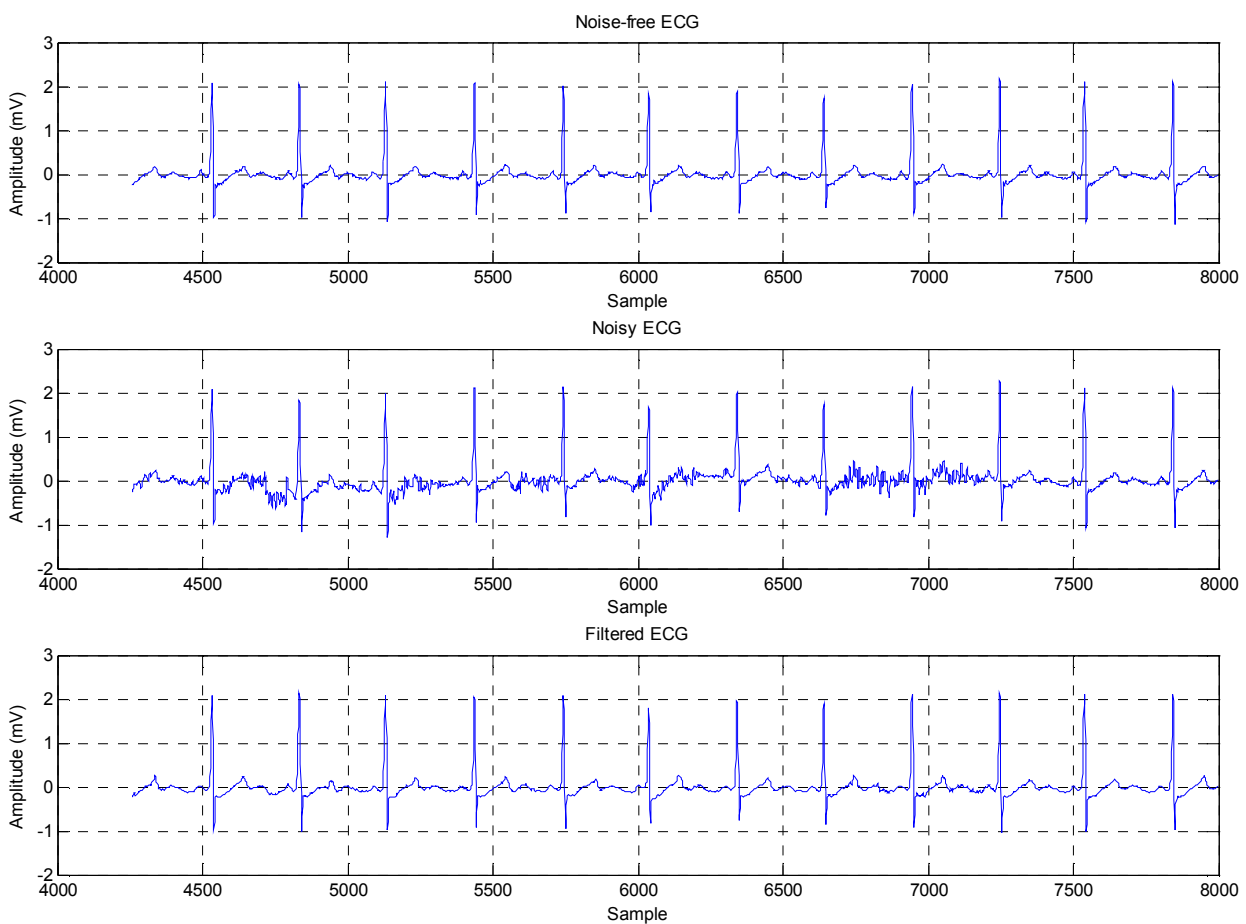

Figure 7.8 Muscle (EMG) artifact removal
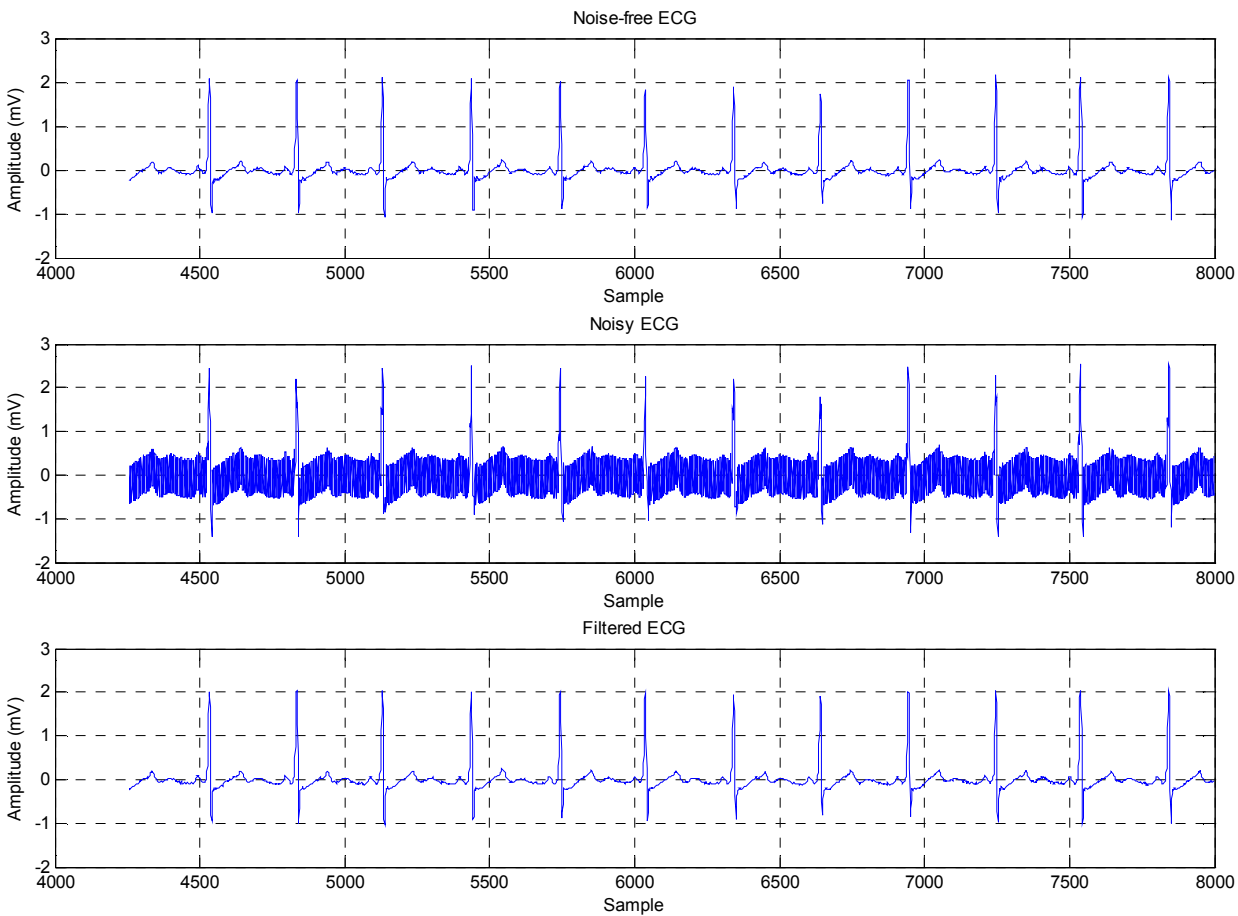

Figure 7.9 The $60 \mathrm{~Hz}$ power-line removal 

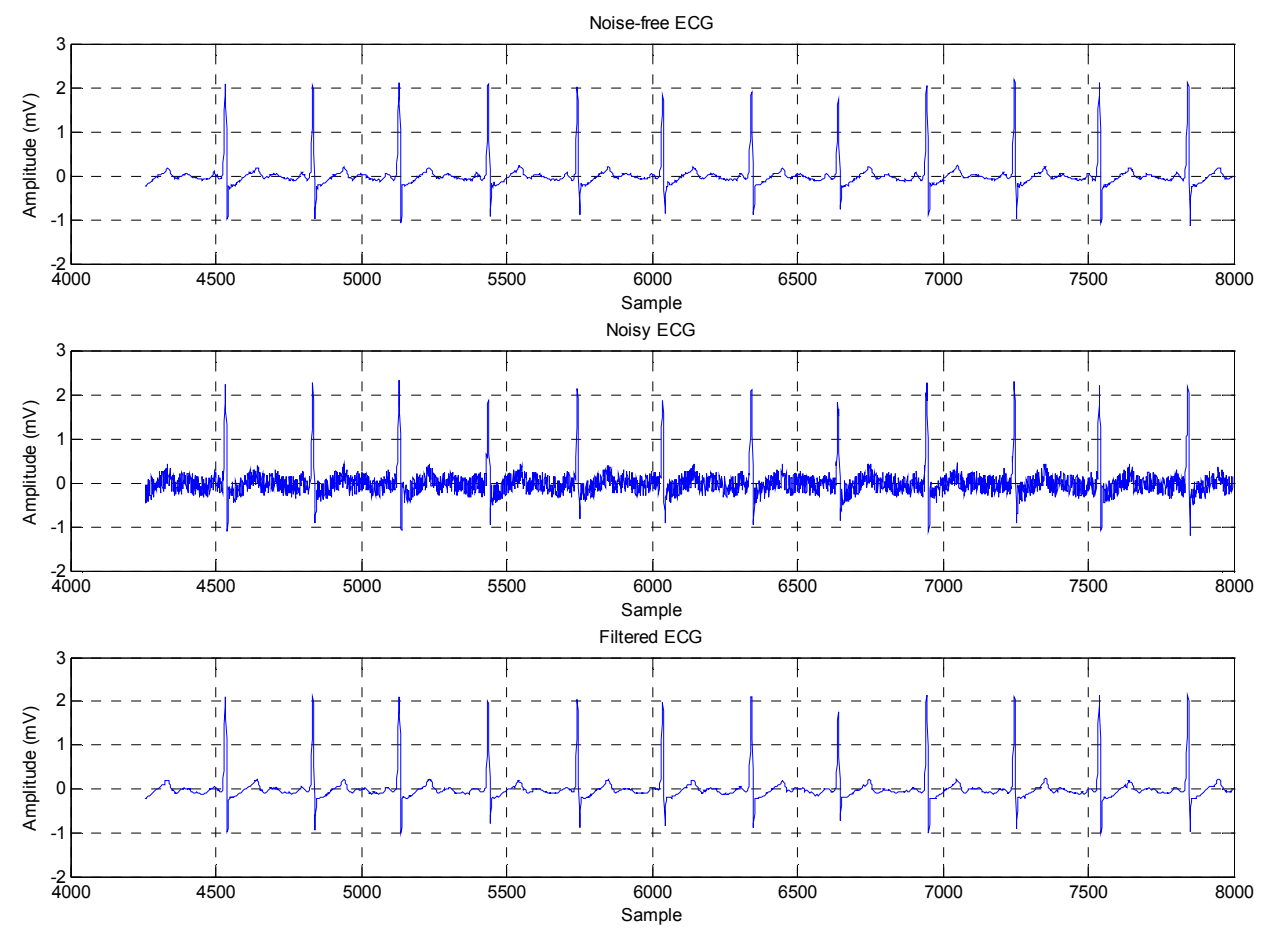

Figure 7.10 Gaussian white noise removal

\subsubsection{Removal of combined noises}

The experimental results from the previous section show that the proposed method was able to perform ECG noise reduction on individual noise with an acceptable margin. In this section, the experiment will test the network's performance when dealing with a combination of all noises. In addition, the network is trained on different ECG records. This is important because a different patient has a different ECG shape. In fact, it is the difference in shape that allows a doctor to perform the patient's diagnosis. Patients with healthy hearts usually have a similar shape and characteristic throughout. The more uniform the shape, the easier the network is able to perform noise reduction. Even 
among patients with consistency in ECG shape, one patient's ECG may have sharper spits that closely resemble electrode motion while others may have a smoother curve. In this case, the filtering result will be different from patient to patient. In the situation where a patient is suffering from a severe heart-related disease, the ECG shape will alter dramatically as shown in figure 7.11. Such abnormal behavior of ECG will make it extremely difficult for the network to learn. However, for the purpose of noise reduction implemented in this paper, all ECGs used in the experiment are assumed to have a typical shape. Though this problem can be generally solved by introducing additional nodes to the hidden layers or even adding an extra layer and then fine-tuning the network for optimal results; however, for the sake of simplicity, the demonstration will be focusing on a more simple and uniform signal (figure 7.12).
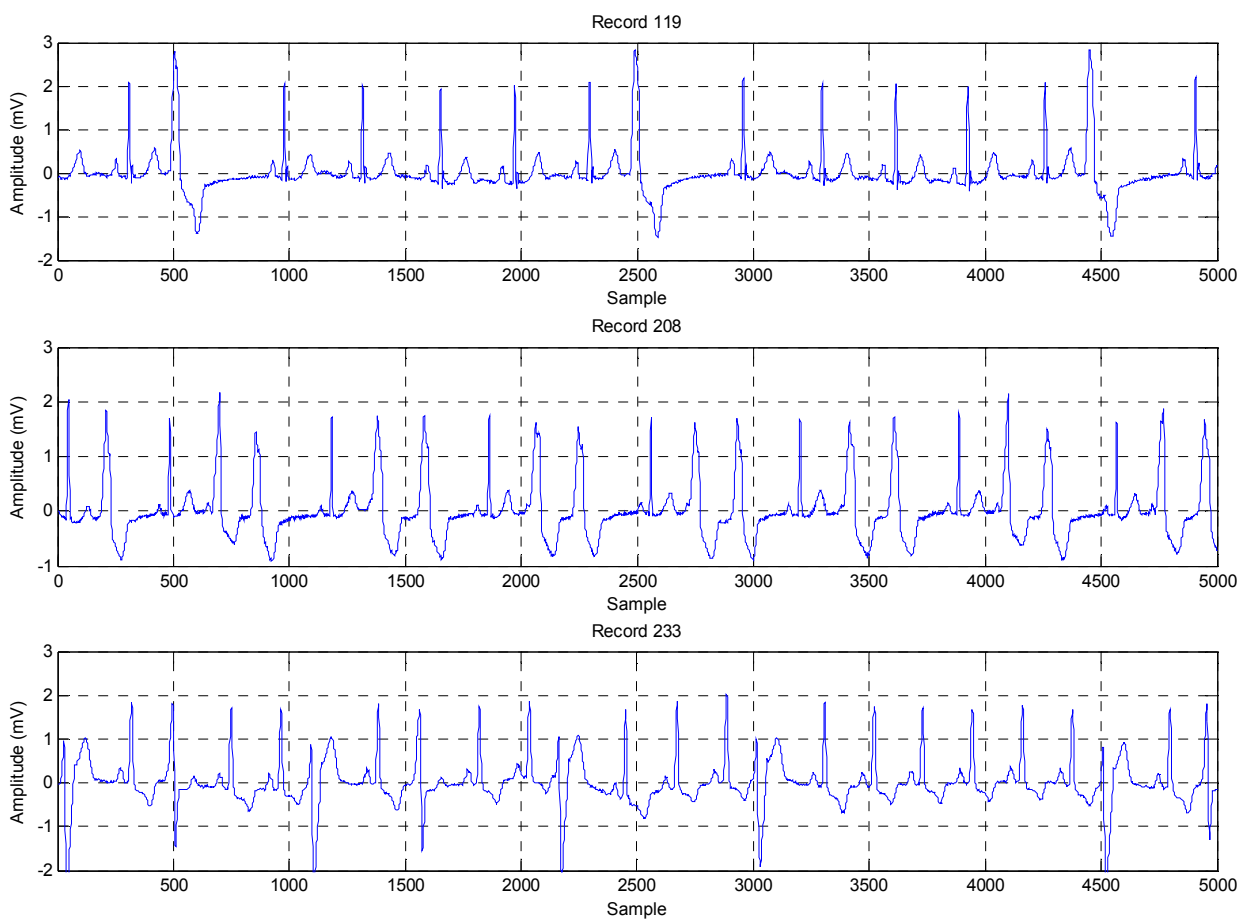

Figure 7.11 ECG with irregular shape taken from [30]. Record: 119, 208, 233 
In this experiment, noise reduction will be performed on three ECG records: 103,115 , and 220. Noise-free versions of all three records are shown Figure 7.12. The three particular signals are chosen because of their distinctive behavior. The success of noise reduction depends on how well a neural network can understand the basic characteristics of the ECG signal. Some ECG signals may be easy for the network to learn while others may be more difficult. Hence, it is important to try out at least a few different types of ECG. Record 103 has smooth and curvy characteristics while Record 220 consists of sharp triangular spits. Record 115 has a very low profile when compared to Record 103 and 220. In addition to this, the network is also tested for consistency by training and testing it 10 times. Example results of noise reduction for three records are shown in figure 7.13-15. Table 7.2 provides the SNR improvement of each 10 tests for three ECG signals over the noisy ECG's SNR display on the very top row. The average SNR improvement can be found on a very last row of each ECG record column.

Table 7.2 Performance consistency in term of SNR improvement

\begin{tabular}{|l|r|r|r|}
\hline \multicolumn{1}{|c|}{ Test \# } & $\begin{array}{c}\text { ECG Record } \\
\mathbf{1 0 3}\end{array}$ & $\begin{array}{c}\text { ECG Record } \\
\mathbf{1 1 5}\end{array}$ & $\begin{array}{c}\text { ECG Record } \\
\mathbf{2 2 0}\end{array}$ \\
\hline Original noisy SNR & $8.98-9.23 \mathrm{~dB}$ & $10.11-10.56 \mathrm{~dB}$ & $9.39-10.04 \mathrm{~dB}$ \\
\hline 1 & $12.22 \mathrm{~dB}$ & $11.82 \mathrm{~dB}$ & $16.46 \mathrm{~dB}$ \\
\hline 2 & $11.99 \mathrm{~dB}$ & $12.89 \mathrm{~dB}$ & $15.22 \mathrm{~dB}$ \\
\hline 3 & $12.23 \mathrm{~dB}$ & $11.98 \mathrm{~dB}$ & $15.77 \mathrm{~dB}$ \\
\hline 4 & $10.17 \mathrm{~dB}$ & $12.61 \mathrm{~dB}$ & $16.07 \mathrm{~dB}$ \\
\hline 5 & $12.00 \mathrm{~dB}$ & $12.37 \mathrm{~dB}$ & $14.40 \mathrm{~dB}$ \\
\hline 6 & $12.24 \mathrm{~dB}$ & $11.76 \mathrm{~dB}$ & $16.13 \mathrm{~dB}$ \\
\hline 7 & $11.34 \mathrm{~dB}$ & $10.85 \mathrm{~dB}$ & $15.76 \mathrm{~dB}$ \\
\hline 8 & $12.52 \mathrm{~dB}$ & $10.87 \mathrm{~dB}$ & $16.22 \mathrm{~dB}$ \\
\hline 9 & $11.61 \mathrm{~dB}$ & $12.92 \mathrm{~dB}$ & $16.32 \mathrm{~dB}$ \\
\hline 10 & $13.01 \mathrm{~dB}$ & $12.68 \mathrm{~dB}$ & $14.82 \mathrm{~dB}$ \\
\hline Average & $11.93 \mathrm{~dB}$ & $12.08 \mathrm{~dB}$ & $15.71 \mathrm{~dB}$ \\
\hline
\end{tabular}



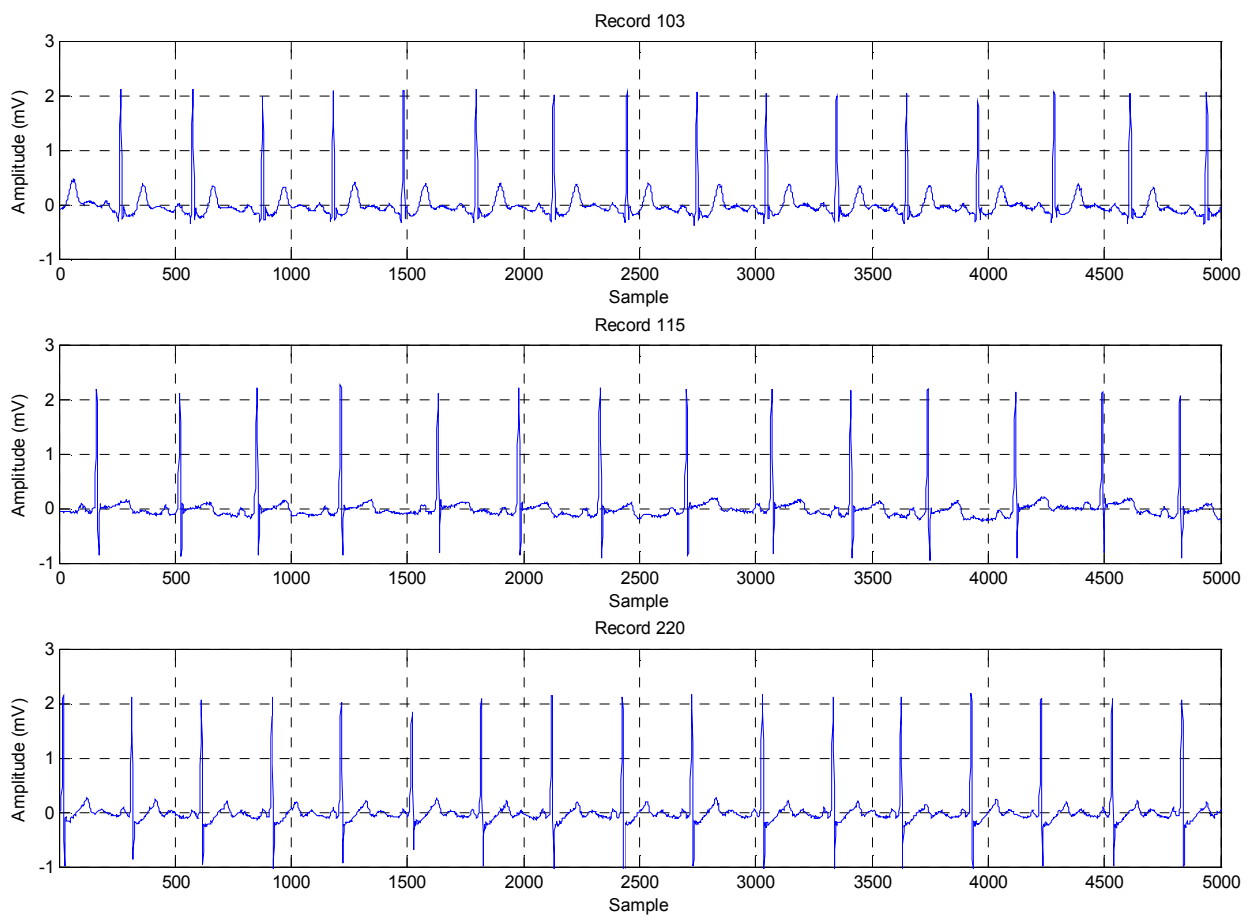

Figure 7.12 Three ECG records taken from [30]: 103, 115, and 220
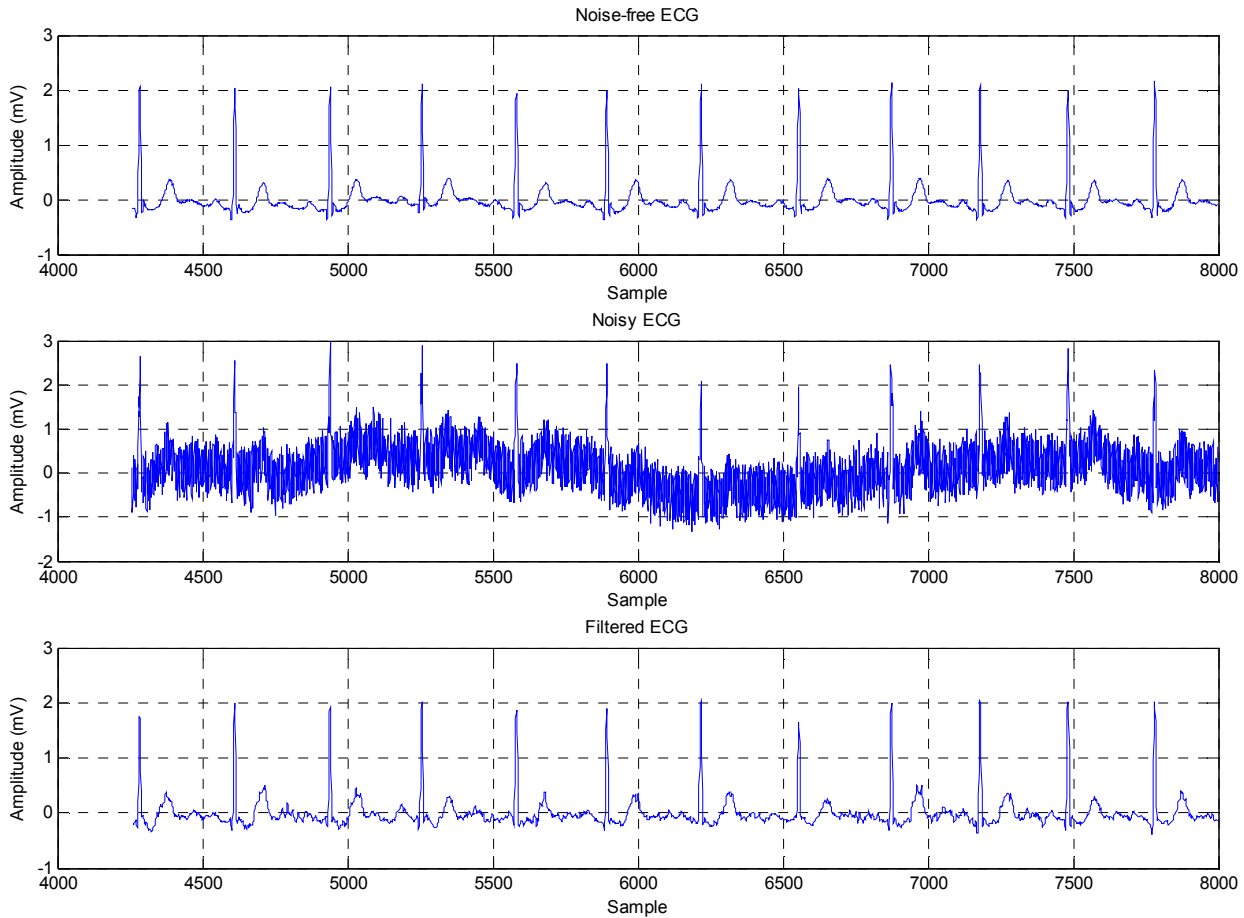

Figure 7.13 Noises combined removal using patient's ECG record 103 

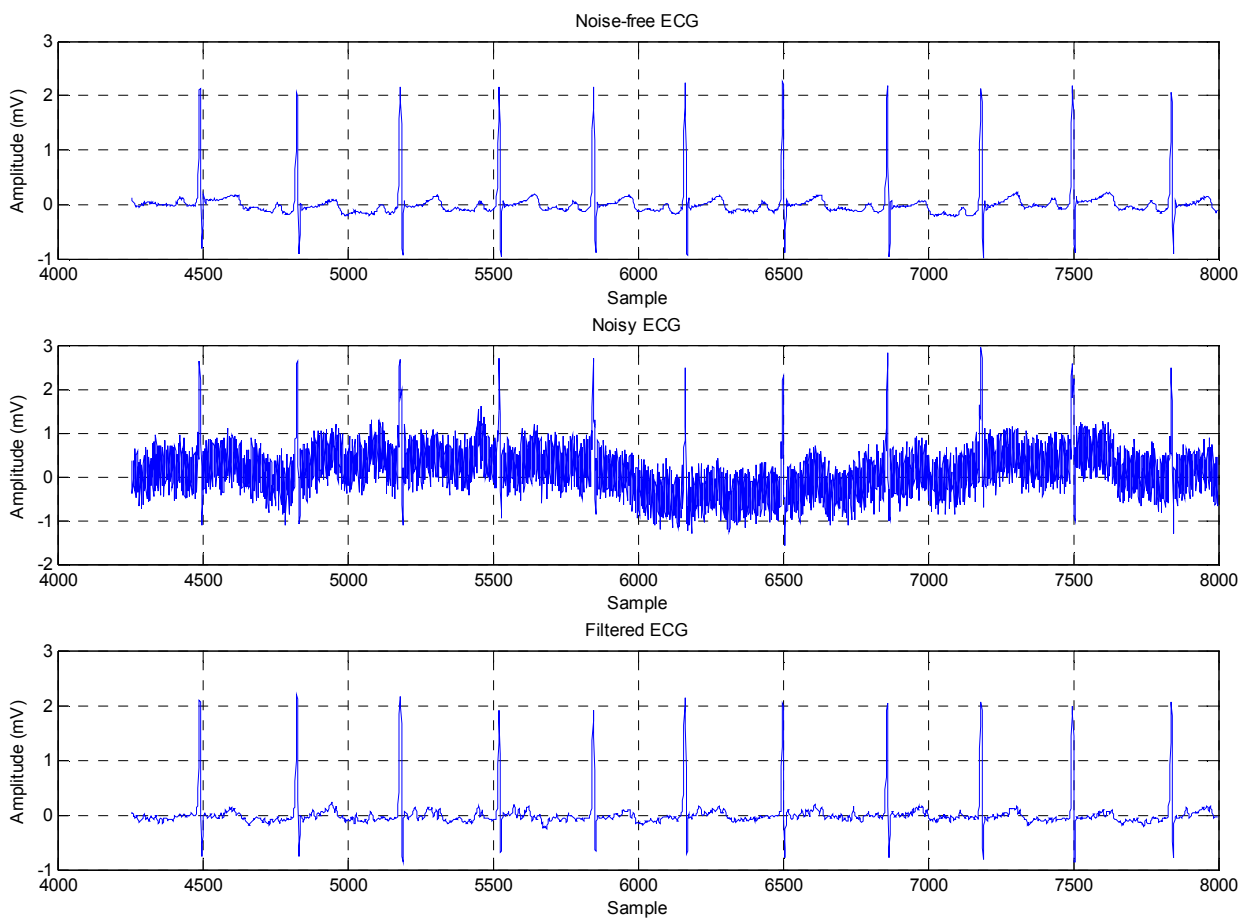

Figure 7.14 Noises combined removal using patient's ECG record 115
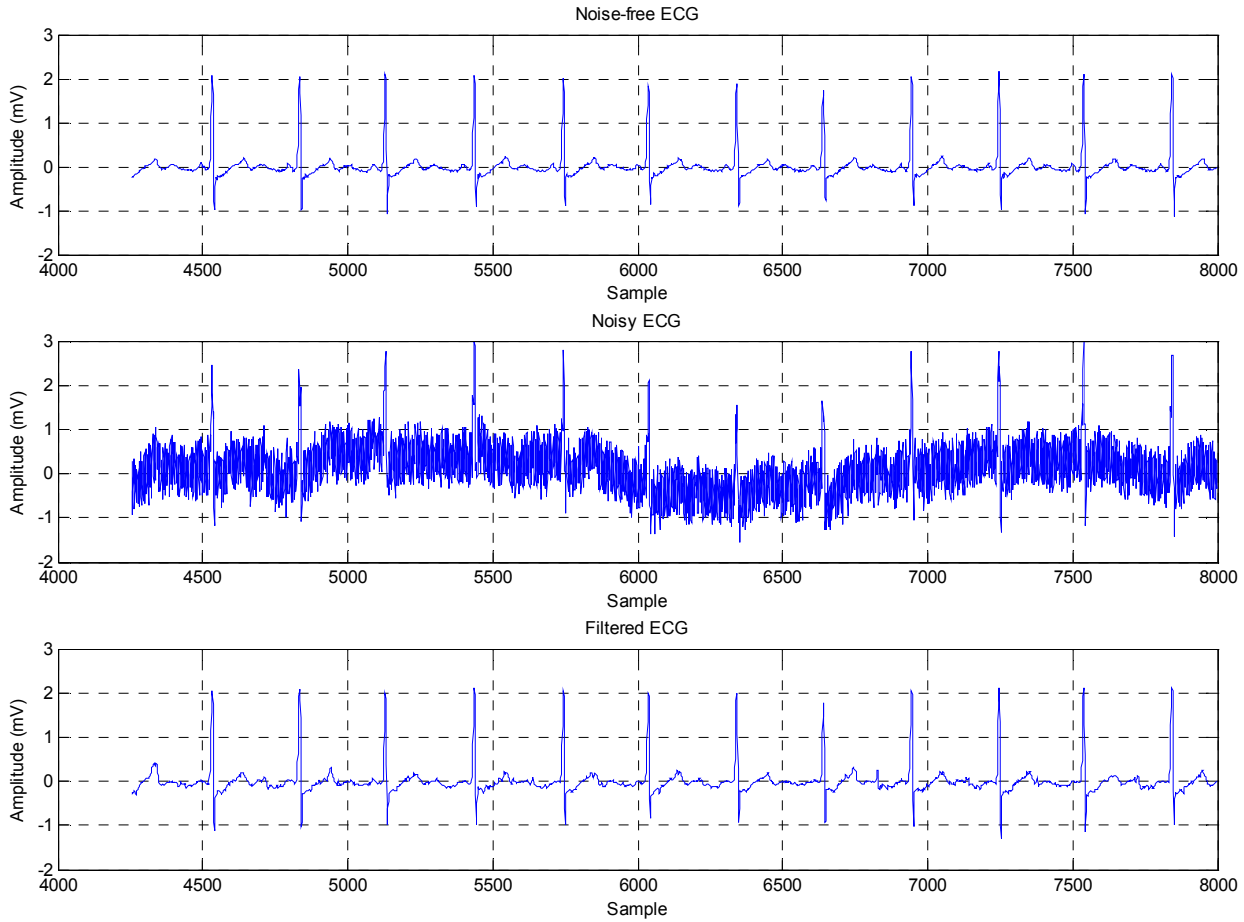

Figure 7.15 Noises combined removal using patient's ECG record 220 
From table 7.2, not only the network manages to remove noises from ECG, it is also able to prove consistency over 10 consecutive runs. However, it is obvious that the level of SNR improvement depends highly on the shape of ECG. For this particular comparison, ECG record 220 is most suitable for the current class of neural networks $\left(N_{64,56,12,10}\right)$ with average SNR improvement of $15.7 \mathrm{~dB}$ while ECG record 103 and 115 gain about $12 \mathrm{~dB}$. As stated earlier, this is because the fine-tuning of the network was done with record 220. The number of hidden nodes may not be sufficient enough for the network to work with record 103 and 115 . If this is really a case, adding more nodes to the network should improve SNR performance. To do this, a larger class of neural network $\left(N_{64,60,15,10)}\right.$ is trained on the on ECG record 103 and 115 . Figure 7.16-17 provide input-output results from the testing phases for both records. The original noise-free ECG is shown in green, noisy signal is in blue, and filtered signal is red. For better understanding, SNR is also calculated from the average of 5 runs, shown in table 7.3. Indeed, the average improved SNR are higher than that in table 7.2. SNR of record 103 improves $0.85 \mathrm{~dB}$ and record 115 improves $1.02 \mathrm{~dB}$. Because only 7 nodes were added to the previous class of neural network, such a small improvement is expected.

Table 7.3 SNR improvement with larger training network

\begin{tabular}{|l|r|r|}
\hline \multicolumn{1}{|c|}{ Test \# } & ECG Record 103 & \multicolumn{1}{c|}{ ECG Record 115 } \\
\hline Original noisy SNR & $8.98-9.29 \mathrm{~dB}$ & $9.92-10.24 \mathrm{~dB}$ \\
\hline 1 & $12.35 \mathrm{~dB}$ & $13.37 \mathrm{~dB}$ \\
\hline 2 & $12.93 \mathrm{~dB}$ & $12.85 \mathrm{~dB}$ \\
\hline 3 & $13.12 \mathrm{~dB}$ & $13.07 \mathrm{~dB}$ \\
\hline 4 & $12.50 \mathrm{~dB}$ & $13.24 \mathrm{~dB}$ \\
\hline 5 & $13.01 \mathrm{~dB}$ & $12.98 \mathrm{~dB}$ \\
\hline Average & $12.78 \mathrm{~dB}$ & $13.10 \mathrm{~dB}$ \\
\hline
\end{tabular}




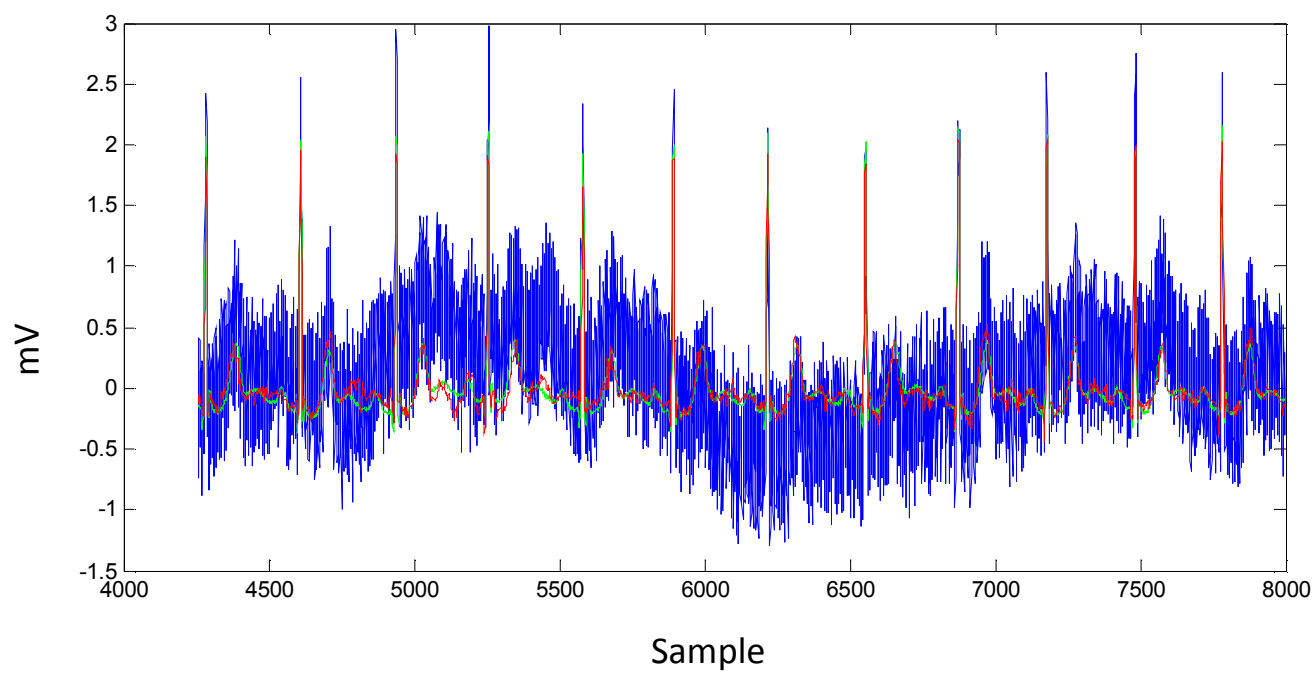

Figure 7.16 Test result of ECG record 103 from re-training with larger network

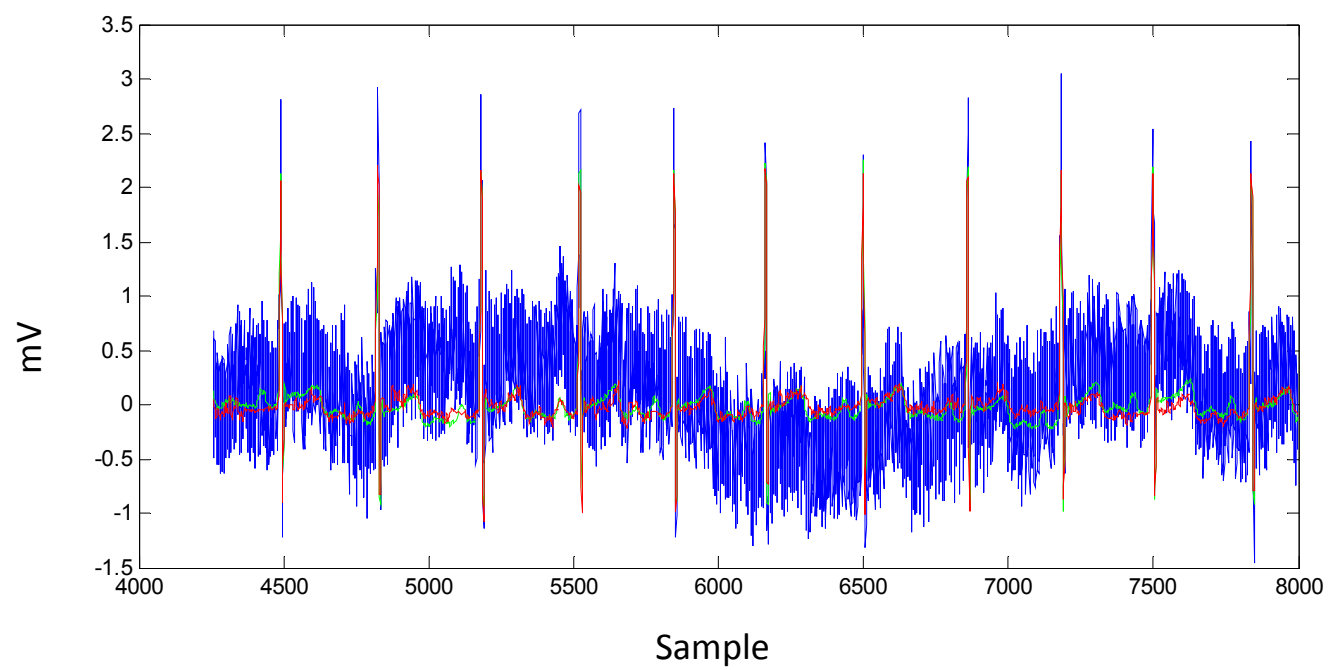

Figure 7.17 Test result of ECG record 115 from re-training with larger network

More hidden nodes contribute to better filtering results. This, however, does not come without a cost. Adding more nodes to the network also means that additional training time is needed. Typically, more nodes usually yield better results; however adding large numbers of nodes is not desirable. The structural outline of the neural network must be balanced between the number of nodes and training time. The balance between more 
nodes and training time is only a matter of fine-tuning the system by adding and/or removing nodes from the network to achieve optimum performance.

\subsubsection{Effect of DWT and coefficient threshold on the signal}

The previous section had shown the ability of the proposed method to the removed ECG noises and improving overall SNR. Noisy ECG is transformed into coefficients which then are fed to a neural network. The main purpose of running the signal through a DWT is mainly for bandwidth decomposition in which neural network can better process the signal. But during this process, coefficient thresholding is also performed. As a result, high frequency noise is removed. From this, one can see that coefficient thresholding has its part in removing noise. In this section, DWT and thresholding are to be examined by inspecting the signal before and after these steps and analyzing their impact on noise reduction.

A general DWT transformation of an ECG signal (record 220) for each level is shown in figure 7.18. The very top signal is the original noisy ECG. The next following 8 plots $\left(d_{1-8}\right)$ are decomposed signals of the wavelet coefficient. Lastly, the very bottom signal is a scaling signal. For better visualization of the thresholding process, both wavelet and scaling coefficients are chained into on long series shown figure 7.19 with the right hand side representing higher bandwidth and to the left representing lower bandwidth. As one can see, there are fewer fluctuations on the right side when comparing to the left side. This is because all the coefficients on the right half consist of only white noise. On 
Wavelet Decomposition
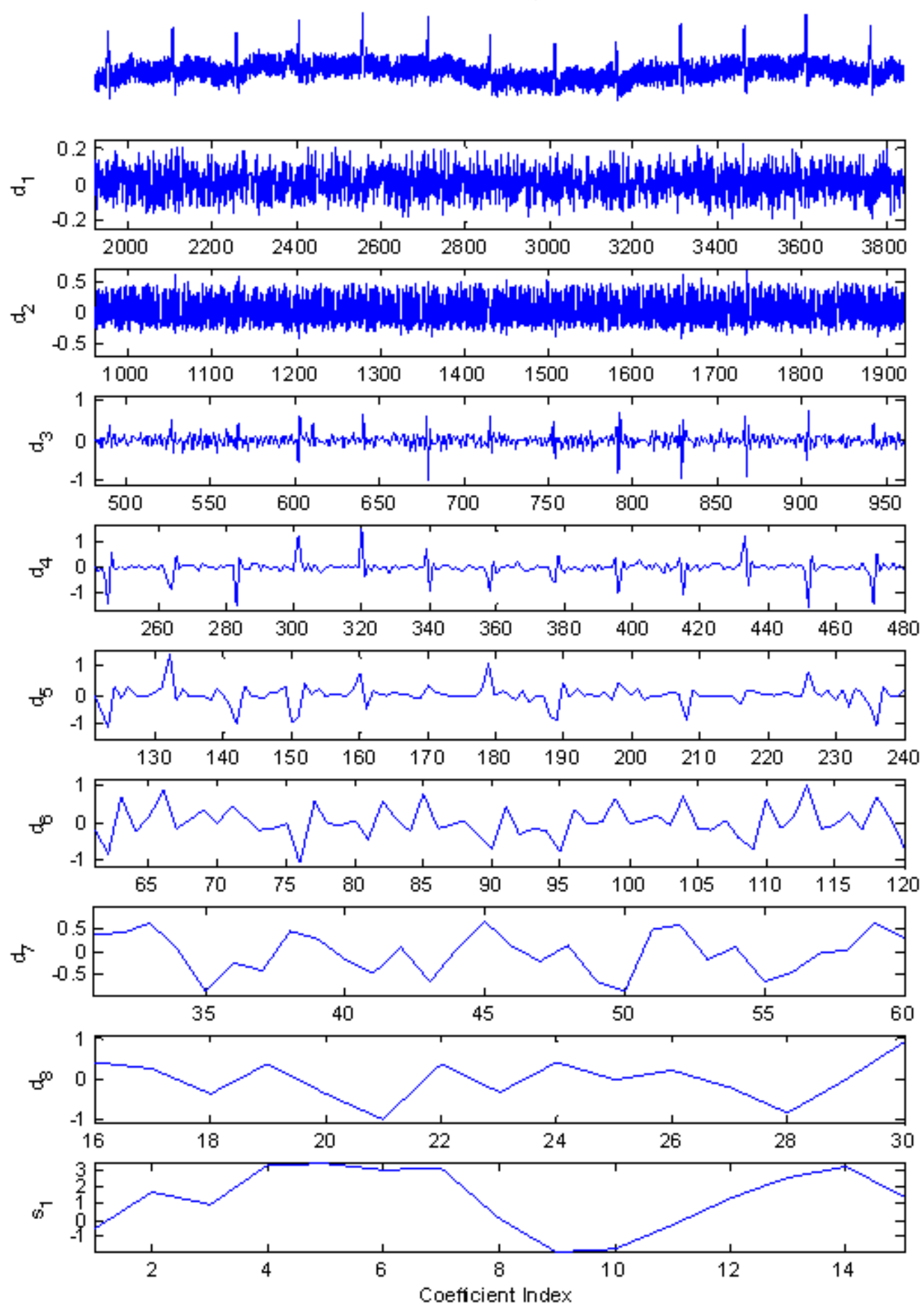

Figure 7.18 Wavelet decomposition of 8 levels 
the other hand, the left half of the coefficients contain many interesting fluctuations in amplitude. This is because they contain an ECG signal as well as a baseline drift, EMG, electrode motion artifact, and $60 \mathrm{~Hz}$ power-line noises. It is apparent that the signals of interest are contained only in the left half of the coefficients and only this half is to be processed by a neural network. By looking at the coefficients alone, it is difficult to understand what the left half of the signal look like. Inversing the coefficients

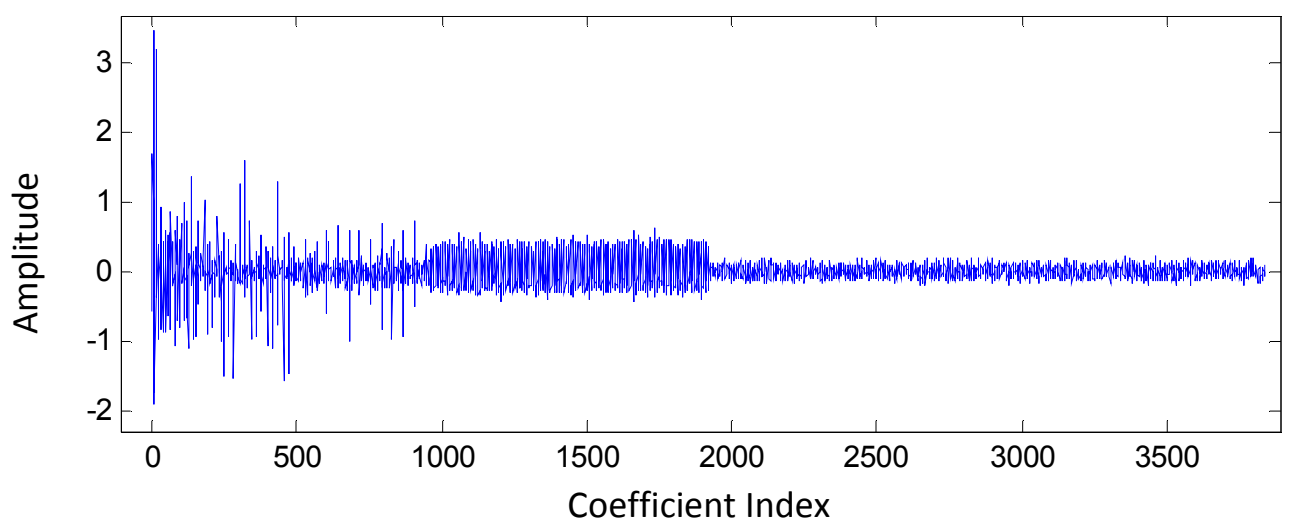

Figure 7.19 Noisy ECG coefficients

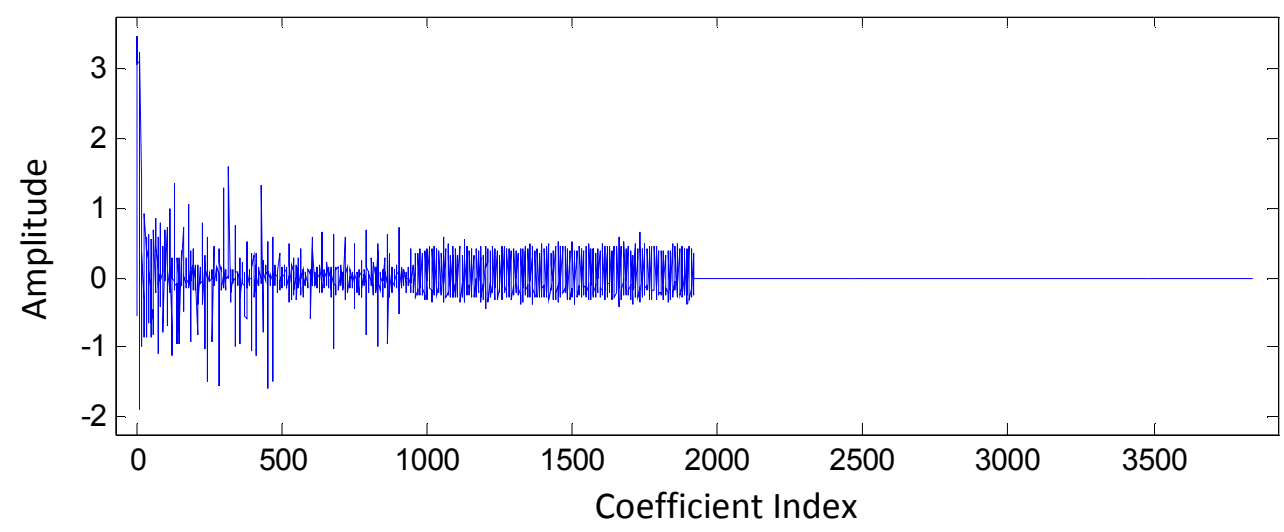

Figure 7.20 Removing coefficients at high frequency 


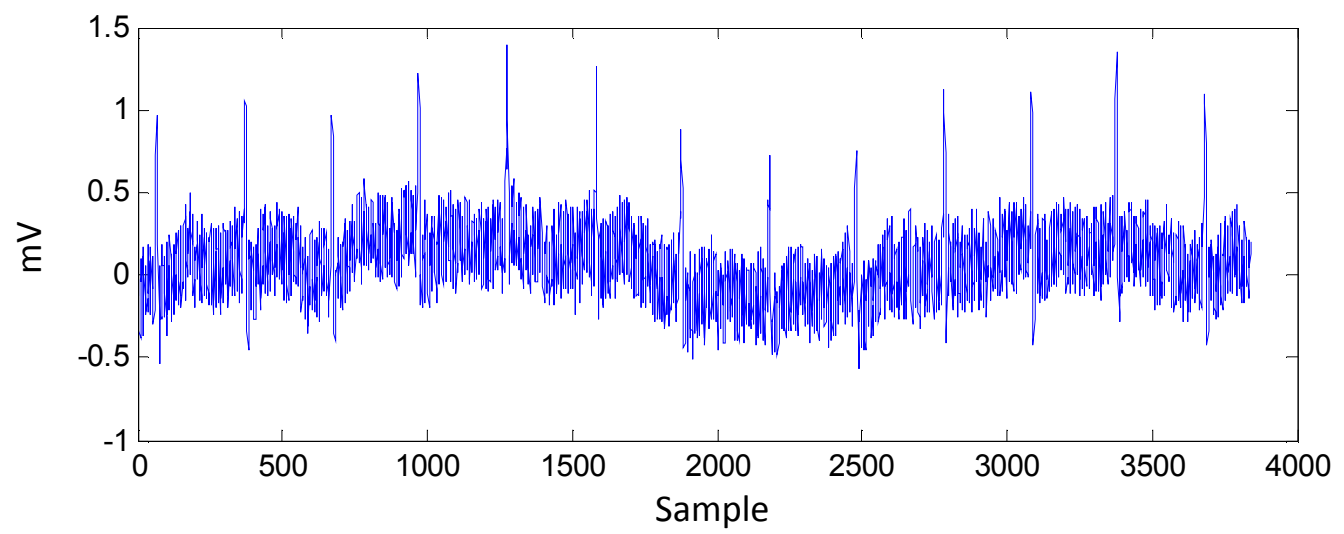

(a) Noisy ECG before thresholding

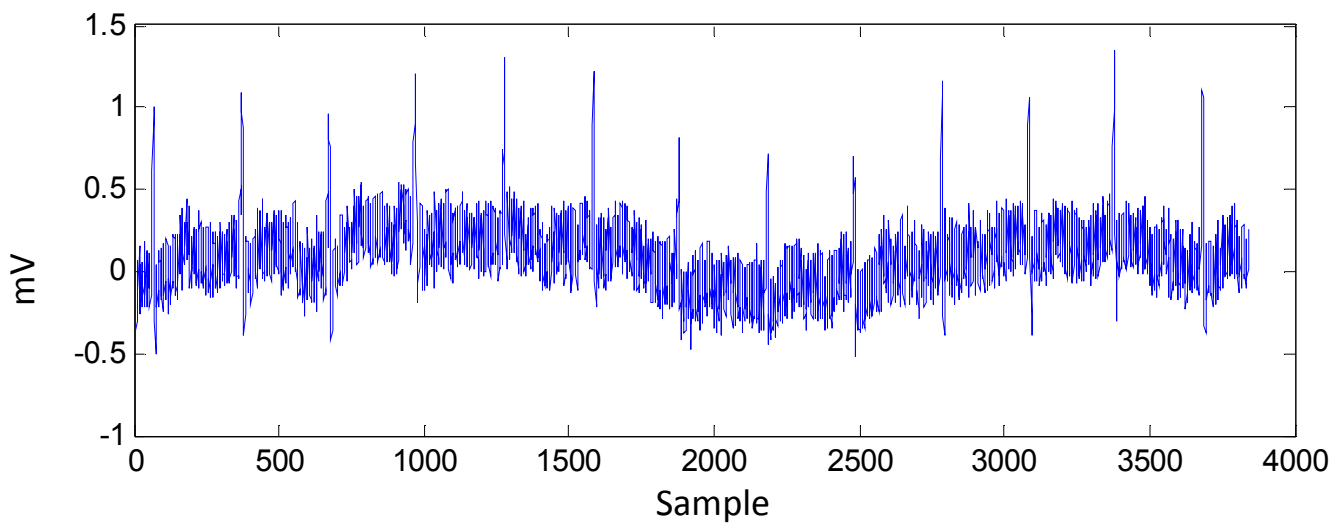

(b) Noisy ECG after thresholding

Figure 7.21 Comparing noisy ECG signal prior and after the thresholding

back to the original signal can help understanding visually what is actually given to neural network. To do so, first, the right half is set to zero. In DWT, setting any coefficient to zero is the same process as discarding it, see figure 7.20. A comparison between noisy ECGs before and after thresholding is shown in figure 21.

Visually, the comparison between the noisy ECG before threshold (figure 21.a) and noisy ECG after threshold (figure 21.b) shows no differences. Only through the SNR calculation is the difference revealed. The SNR of the original signal is about $9.82 \mathrm{~dB}$, while the processed signal has $10.11 \mathrm{~dB}$ which translates to less than $0.3 \mathrm{~dB}$ improvements. The 
improvement is very small and insignificant. One may ask: why then include the threshold? The significance of the threshold does not lie on how much noise is removed but how many coefficients it eliminates without affecting the ECG signal. As stated earlier in this chapter, the step the threshold takes is not for noise removal but for removing unnecessary coefficients and reducing the number of neural network inputs needed to process the information. Noise reduction is simply a byproduct of such process.

\subsubsection{Comparison with Transitional Methods}

The filtration of the traditional methods consists of four different linear filters: Lowpass filter (LPF), High-pass filter (HPF), Notch filter, and Signal averaging. All four filters are chained in cascaded form with setting shown in table below:

Table 7.4 Filters setting

\begin{tabular}{|l|r|r|}
\hline \multicolumn{1}{|c|}{ Filter } & Cut-off Frequency & \multicolumn{1}{c|}{ Order } \\
\hline Low-pass & $90 \mathrm{~Hz}$ & 501 \\
\hline High-pass & $0.5 \mathrm{~Hz}$ & 500 \\
\hline Notch & $55 \mathrm{~Hz}, 65 \mathrm{~Hz}$ & 500 \\
\hline Signal Avg. & $\mathrm{N} / \mathrm{A}$ & 5 \\
\hline
\end{tabular}

LPF and HPF serve as band pass filters allowing only signal within ECG frequencies band to pass through. A notch filter is used to remove $60 \mathrm{~Hz}$ power line noise. Lastly, signal averaging will be performed to smooth the overall signal. The original ECG signal is contaminated with baseline wander, muscle (EMG) artifact, electrode motion, $60 \mathrm{~Hz}$ power-line, and white noise. SNR of the noisy signal is measured to be $9.63 \mathrm{~dB}$. After filtering the signal, the SNR is $16.15 \mathrm{~dB}$. Despite this improvement, the filtered signal is 

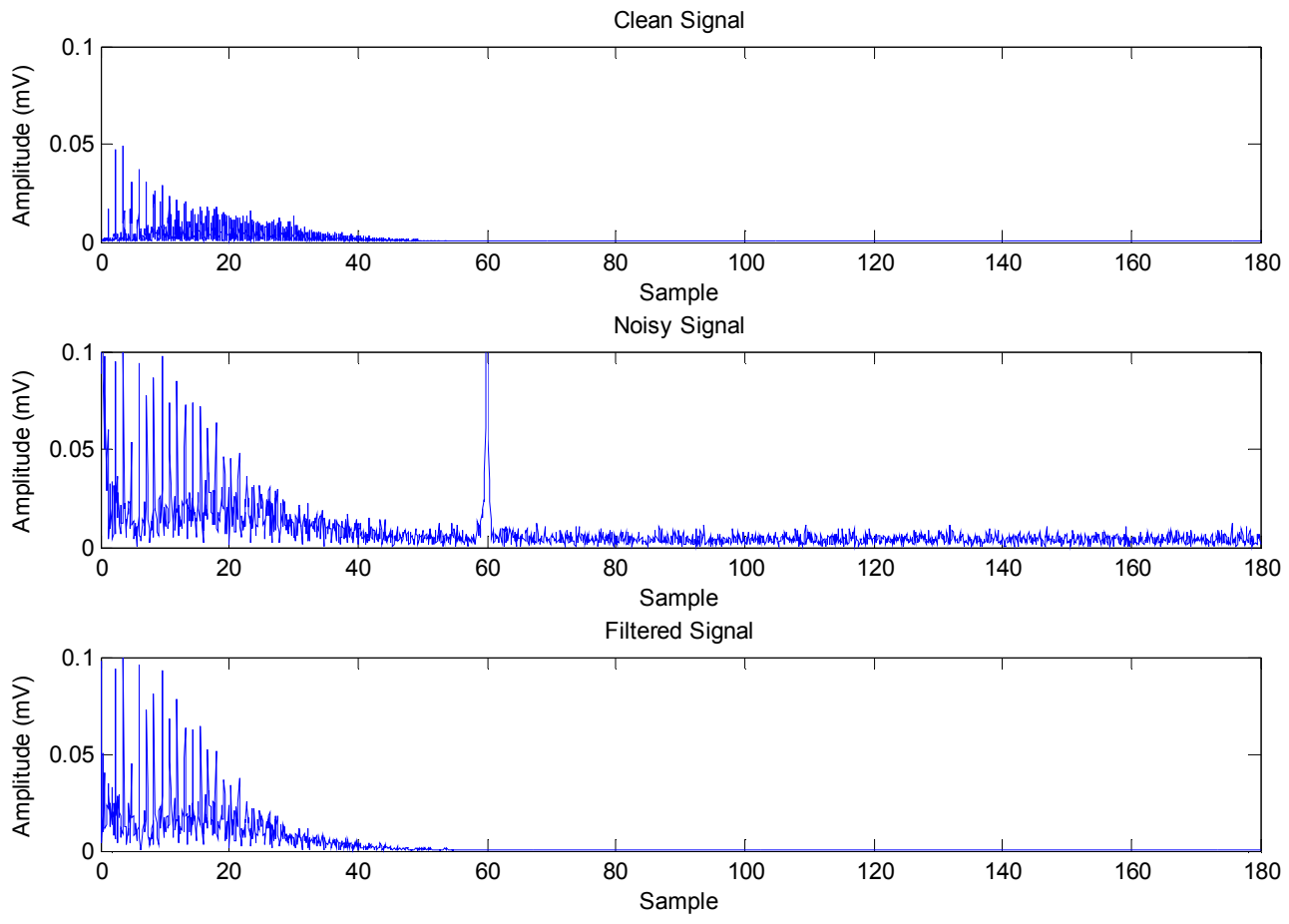

Figure 7.22 Frequency analysis of signal prior and after filtering
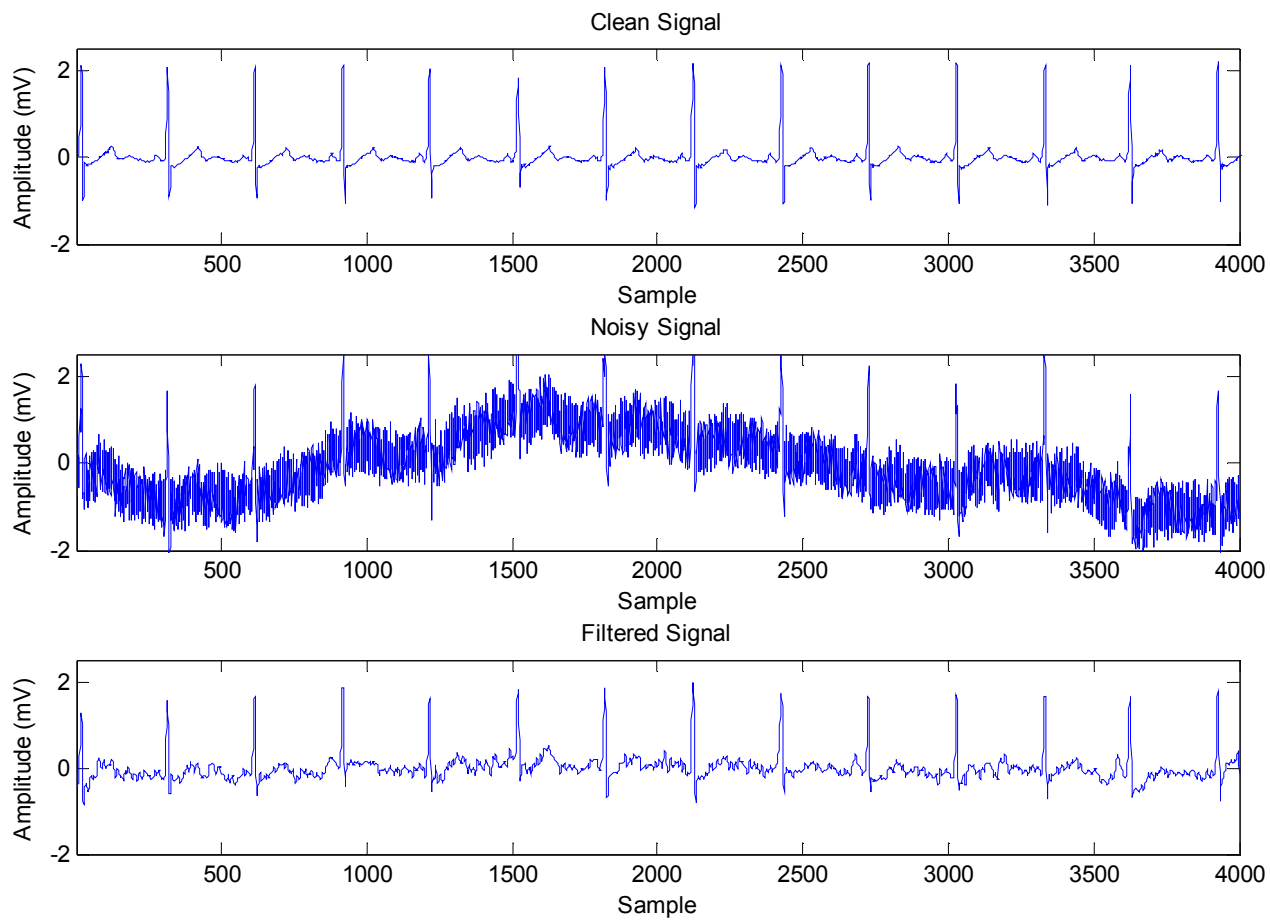

Figure 7.23 ECG signals in time domain 
still suffered from EMG and electrode motion since none of traditional methods are capable of removing them. Figure 7.2 provides the comparison between clean, noisy, and filtered signals, respectively, in frequency domain. The result from adding noises to the ECG can be clearly seen. Due to baseline wander, EMG, and electrode motion, the amplitude at the low frequencies is more visible. At $60 \mathrm{~Hz}$, there is power line interference. And finally, the effect of wide frequencies noise band of white noise expand throughout the frequencies range. After passing the signals through linear filters, low frequency noise at the baseline is reduced, $60 \mathrm{~Hz}$ power line noise disappears, and the effect of the white noise seems to be eliminated. But because of the frequency overlap of EMG and electrode motion, the traditional linear filter methods could not give good results. The inspection of the time domain in figure 7.23 clearly shows the effect of these unfiltered noises.

\subsubsection{Replacement of DWT with LPF and down-sampling}

In this section, the proposed method will be compared with another filtering method that utilizes a similar technique. If the coefficient thresholding in the proposed method is used as a means to reduce the number of inputs to a neural network, then a simple low-pass-filter (LPF) and down sampling technique should do the same thing, and hence avoid the need for DWT. In this section, the evaluation between such methods and the proposed method will be compared.

This alternative design is similar to that in figure 7.3. But instead of a DWT, this alternative design will have LPF and the down-sampling in place of coefficient threshold. 
The LPF removes any frequency of $90 \mathrm{~Hz}$ or higher, followed by down-sampling to cut down the signal, matching the current neural network's input node. Even though the process performed by this alternative method met similar goals to the original design proposed, the following tests, consisting of 5 consecutive runs for each design, proved that the test result of the original proposed method had a better SNR improvement. The result is shown in table 7.5 .

Table 7.5 SNR improvement using LPF and downsampling

\begin{tabular}{|l|r|r|}
\hline \multicolumn{1}{|c|}{ Test \# } & $\begin{array}{c}\text { LFP and Down } \\
\text { sample }\end{array}$ & $\begin{array}{c}\text { DWT and } \\
\text { threshold }\end{array}$ \\
\hline Original noisy SNR & $9.97-9.97 \mathrm{~dB}$ & $9.46-10.02 \mathrm{~dB}$ \\
\hline 1 & $7.38 \mathrm{~dB}$ & $16.11 \mathrm{~dB}$ \\
\hline 2 & $8.23 \mathrm{~dB}$ & $15.75 \mathrm{~dB}$ \\
\hline 3 & $8.01 \mathrm{~dB}$ & $15.92 \mathrm{~dB}$ \\
\hline 4 & $7.21 \mathrm{~dB}$ & $16.32 \mathrm{~dB}$ \\
\hline 5 & $6.93 \mathrm{~dB}$ & $15.44 \mathrm{~dB}$ \\
\hline Average & $7.55 \mathrm{~dB}$ & $15.91 \mathrm{~dB}$ \\
\hline
\end{tabular}

Clearly, SNR improvement of the alternative method is no where match the proposed method. This is mainly because of DWT and its frequency decomposing ability. The key influence of the neural network to learn does not always depend solely on the size of its structure. The pre-process step of transforming the signal into categories of frequencies band such as decomposition can also improve the learning performance of the network as well. In other words, the learning efficiency of neural network improves if its inputs are more organized. This will allow the neural network to determine the relationship between input and output easier. The sample of graphical comparison results are shown 
in figure 7.24 where the blue represents noisy signal, green is noise-free, and red is the filtered signal.

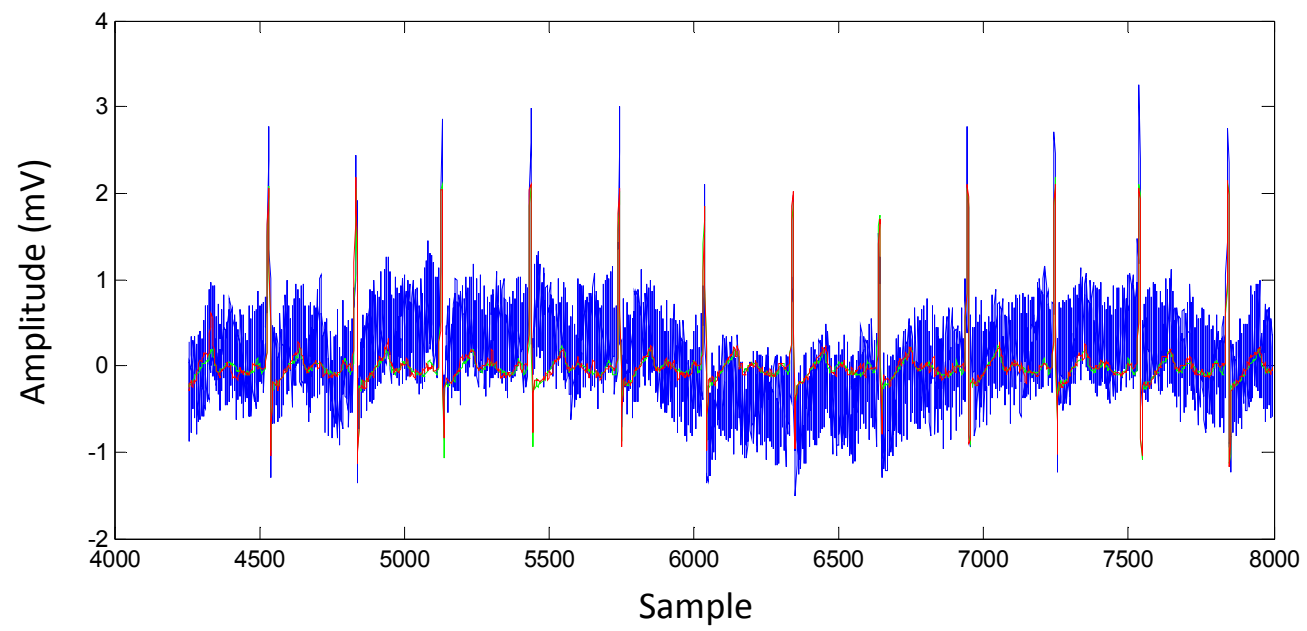

(a) Result for proposed method

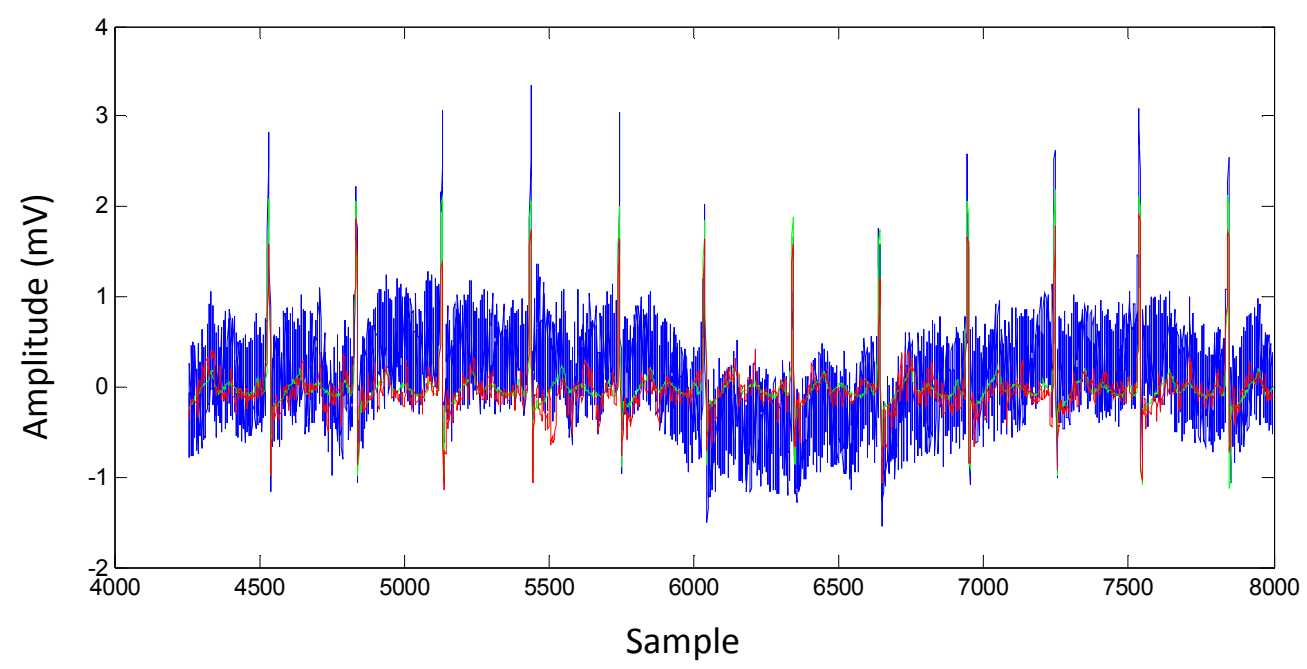

(b) Result from similar alternative method

Figure 7.24 Noise reduction comparisons between the proposed method and a similar alternative method 


\subsection{Conclusion}

The method of wavelet decomposition and artificial neural network presented in this chapter has demonstrated its noise reduction capability of highly non-linear and nonstationary signals through an application of electrocardiogram (ECG). In the specific models given, the delayed values of relevant signals in the system are used as inputs to wavelet decomposition. Its outputs are, then, filtered through the process of coefficient thresholding which removes the majority of coefficients that are not relevant to the signal of interest. Next, these remaining coefficients are used as inputs to a multilayer neural networks. Methods for the adjustment of parameters in generalized neural networks are treated, and the concept of back propagation was introduced in chapter 2 to generate partial derivatives of a performance index with respect to adjustable parameters. From the implementation shown in this chapter, the recovered signals are shown to have good SNR improvement over the contaminated signals. Once trained, the neural network can recognize the wavelet decomposed coefficients of a particular patient and can reconstruct the signal with most of the noises removed. With sufficient training, the network may be applied to process the ECG signals in real-time. 


\section{Chapter 8: Discussion and Conclusions}

In this paper, an alternative method to reduce noises in ECG is proposed that combine wavelet decomposition and neural network. In the last chapter, the effectiveness of this method was demonstrated. This method is superior to conventional methods because it does not depend on the details of the signals studied. In particular, neural network can learn from the sample data during the training session and adapt the basic shape of ECG to reproduce the signal again during the operation. Even with noises such as EMG and electrode motion that have the overlapped frequencies, thus posing serious difficulties for traditional methods, neural network can learn to differentiate differences in signal characteristics and are able to reproduce the signal that closely resembles a noise-free version. But the success of this proposed technique cannot be credited to the neural network alone; wavelet decomposition also plays a major role, aiding the neural network in decomposing a signal into component bands and eliminating most of the unnecessary information for the network to process. As adaptability of neural network gives its capability to learn, it is also the cause of inconsistency during the learning process. This means that the two identical sets of training data are given to the same network, but trained at different times, will not provide the same results. 
Recall from chapter 6 that the LMS algorithm is guaranteed to converge to a solution that minimizes the mean squared error, so long as the learning rate is not too large. This is true because the mean squared error for a single-layer linear network is a quadratic function. The quadratic function has only a single stationary point. In addition, the Hessian matrix of a quadratic function is constant; therefore, the curvature of the function in a given direction does not change, and the function contours are elliptical [42].

Back-propagation is a generalization of the LMS algorithm. Like LMS, it is also an approximate steepest descent algorithm for minimizing the mean squared error. In fact, the algorithm is equivalent to the LMS algorithm when used on a single-layer linear network. When applied to multi-layer networks, however, the characteristics of Backpropagation are quite different. This has to do with the differences between the mean squared error performance surfaces of single-layer linear networks and multilayer nonlinear networks. While the performance surface for a single-layer linear network has a single minimum point and constant curvature, the performance surface for a multilayer network may have many local minimum points, and the curvature can vary widely in different regions of the parameter space. [43].

Due to the nature of neural network, the results they give are not exact but the method is considered to be successful in this particular application if they can provide a solution within a few percentage errors. Developing networks with greater accuracy, most often, corresponds to simply carrying out the training over longer periods (more iterations) 
and/or increasing the number of hidden nodes. For demonstration, the noise reduction method implemented in chapter 7 is applied to one of the patient (record 119) who has irregular cardiovascular which poses difficulty for the current setting used previously. To accommodate for additional work load, the neural network is increased to class $N_{64,64,32,10}$ with double training data and double training iterations. The noisy SNR is measured to be $9.18 \mathrm{~dB}$. After the filtration, the filtered signal has $17.69 \mathrm{~dB}$. Result shown in figure 8.1 provides good noise reduction results.
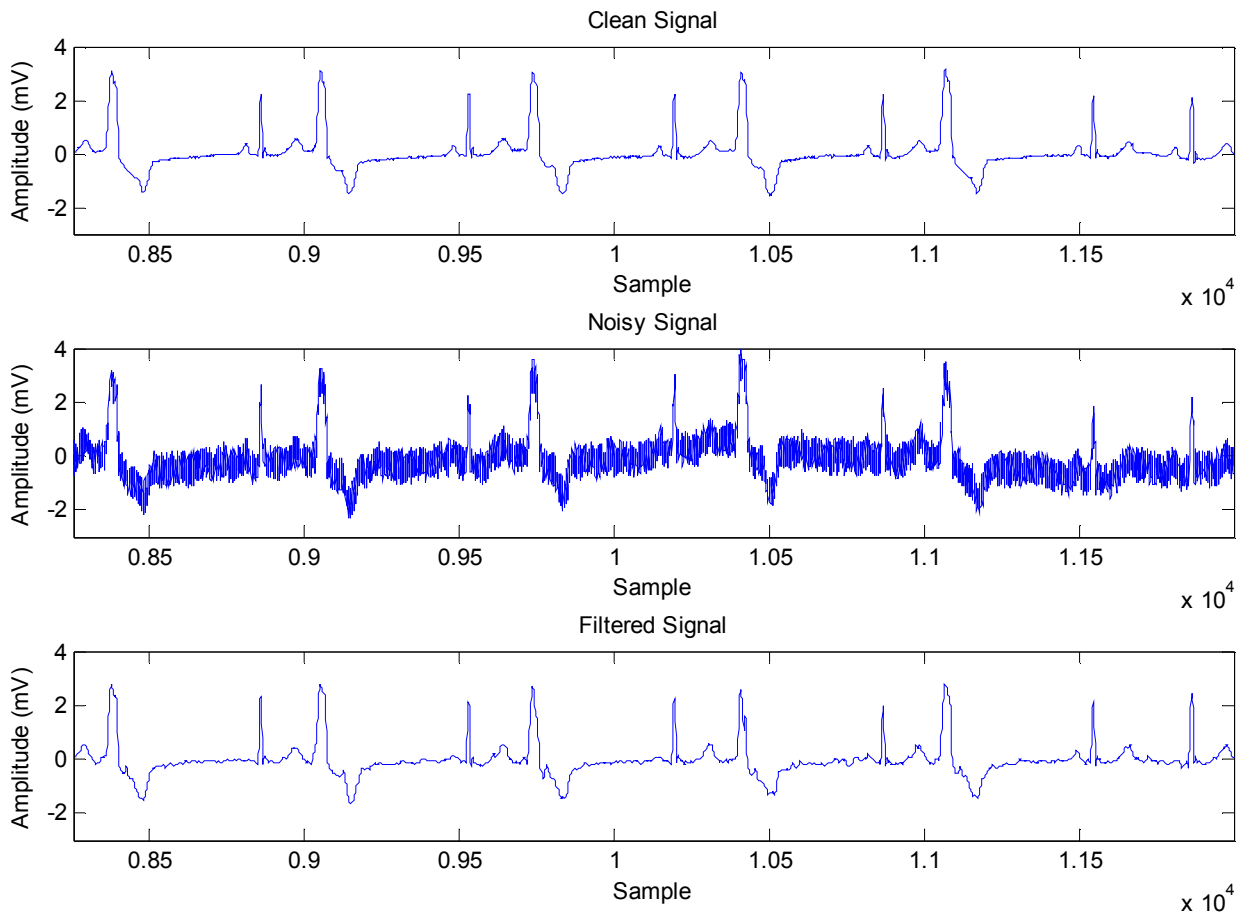

Figure 8.1 ECG noises reduction on record 119

In addition, the filtration is also applied to record 208 and 233 with noisy signals are measured to be 7.91 and 8.65 , respectively. See figure 8.2 and 8.3 . 

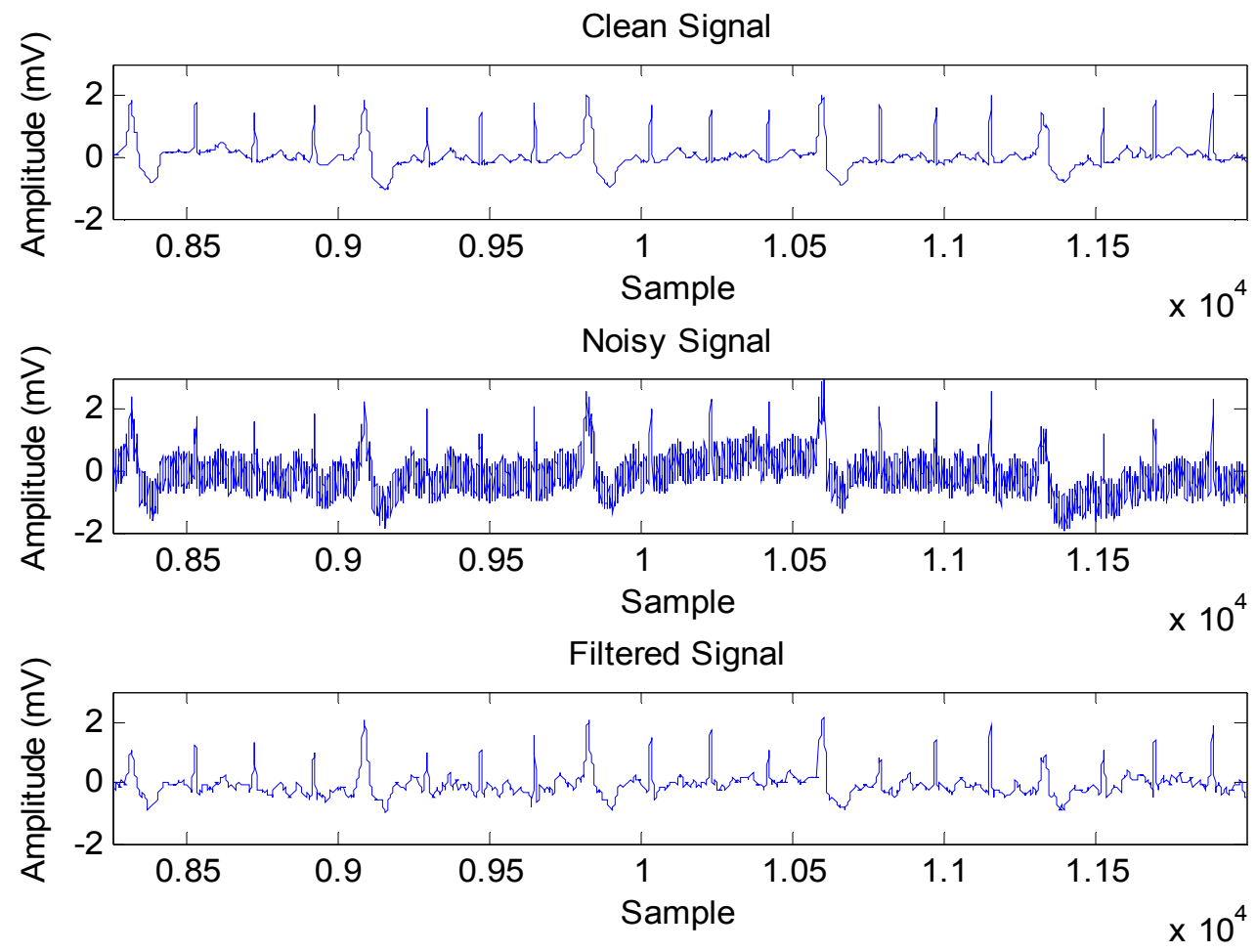

Figure 8.2 ECG noises reduction on record 208
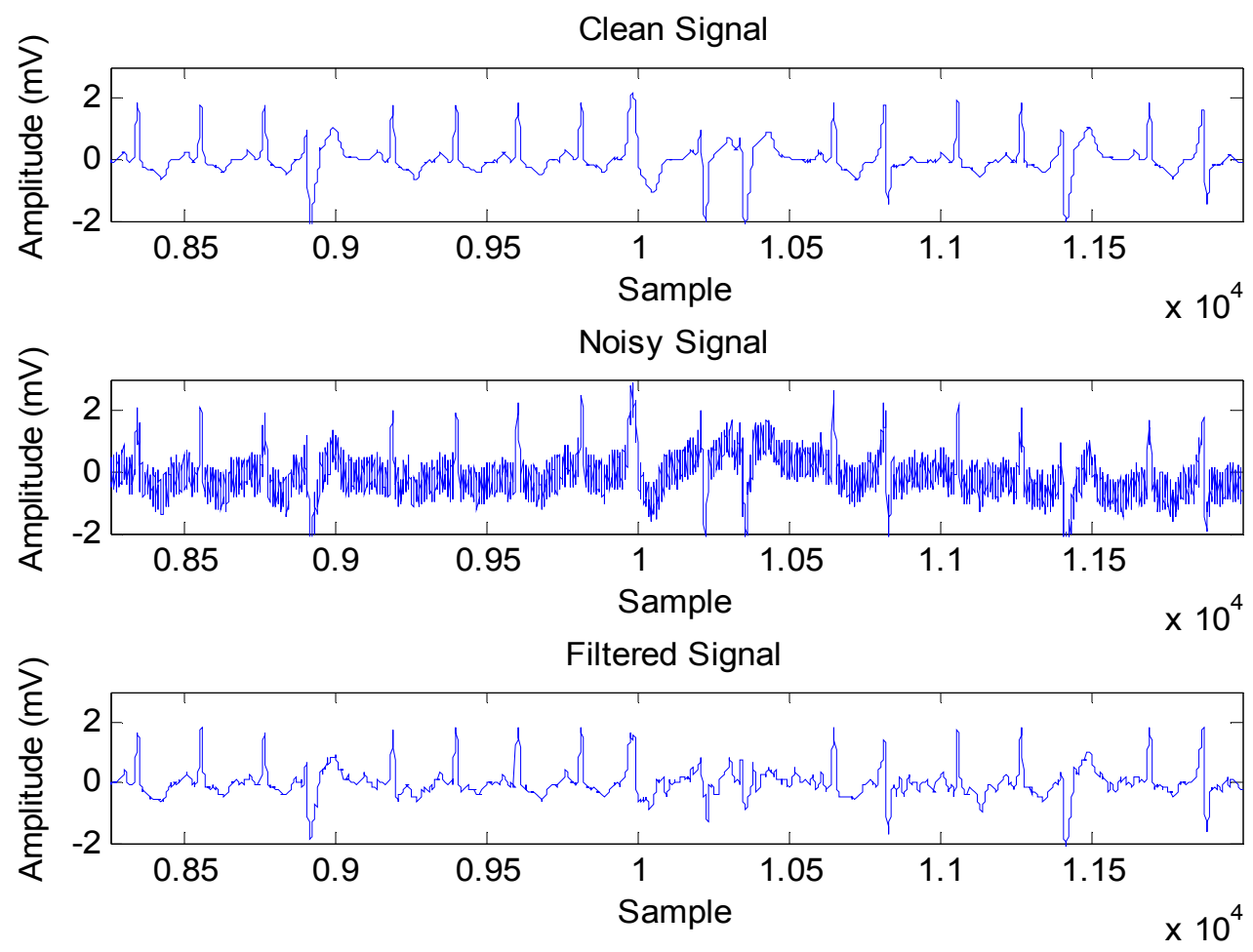

Figure 8.3 ECG noises reduction on record 233 
Unfortunately, the results from record 208 and 233 do not have good improvement as that of record 119. Filtering SNR results are $11.74 \mathrm{~dB}$ for record 208 and $12.23 \mathrm{~dB}$ for record 233. Despite the already increased in resources for neural network, the network could not produce acceptable results. This could due to the fact that the neural network requires even more resources to accommodate for higher complexity of these signals' characteristics. Therefore, providing more hidden nodes and longer training time would be the obvious solution for record 208 and 233.

Adding additional resources to the neural network will increase its potential and improve overall results. However, asymptotic best results achievable are difficult to predict and the expense in computational time may become prohibitive. On top of that, too much training may also cause over-fitting problem. So, choosing the right network size and number of training iterations is a classic problem with all neural networks. It must be noted that the number of iteration applied in previous chapter was determined through trial and error. Because the learning results of neural network are not static and directly dependent on the number of iterations, some $x$ numbers of training will yield different result from the same number in previous learning. The goal here is not to aim for the most optimal results, but rather, the acceptability, as explained previously. In other words, the number of training iterations must guarantee that the network will converge within an "acceptable" range.

Improvement in results also depends on a good training set of data. A neural network is highly dependent to its initial training data. If the fluctuation of the inputs to neural 
network is within some expected boundary, training data can be chosen to cover those ranges. However, due to the nature of biological noises and ECG signal, which varies from one individual to the next, a large sample of training data may be needed from every individual as reference for a neural network.

Neural network training is equivalent to expanding a function in terms of basis functions, where each hidden node is a basis function described, for example, by an optimally parameterized hyperbolic function (or other such lower and upper bounded function). The back-propagation algorithm is an effective means of achieving the parameterization. However, it is not clear if it is the best algorithm to achieve this end. Nevertheless the experimental results from the chapter 7 provided good overall results. Recent study of back-propagation in [42] provided a different alternative variation of the method: Momentum back-propagation (MOBP), Variable learning rate back-propagation (VLBP), and Levenberg-Marquardt back-propagation (LMBP). It will be interesting to see how well they perform in a noise reduction applications.

Apart from all that has mentioned, initialization of network's parameters was the least talked about topic. In many common practices, including this paper, initialization often involves assigning some randomly generated number to every parameter. The hope is that these parameters will slowly converge as the algorithm of back-propagation performs the fine-tuning for each iteration. In [29], a good initialization algorithm can dramatically improve the learning efficiency and reduce the number of training iterations. In the modeling application, [29] had shown that a learning of a function that 
is similar to equation (4.12) can be achieved in as little as 4 iterations. As in the future work, this topic is definitely worth looking into.

This paper proposed a novel dynamical nonlinear wavelet domain coefficient thresholding model and multi-layer neural network for signals of cardiovascular origin to reduce noise in signals. The initial experiment was conducted on a normal ECG with combination of common noises and found the proposed model robust. Experimental results have been evaluated and are confirmed that the technique is able to achieve good SNR. Further, implementation of this algorithm is simple and straight forward. The results are visually pleasant and proved to be superior to conventional methods such as linear filters. The proposed model has potential application in ECG noise reduction applications. 


\section{References}

[1] Hyun Dong Kim, Chul Hong Min, Tae Seon Kim, "Adaptable Noise Reduction of ECG singals for Feature Extraction," J. Wang et al. (Eds.): ISNN 2006, LNCS 3973, pp.586-591, 2006.

[2] Shin'ichi Tamura, Masami Nakamura, "Improvements to The Noise Reduction Neural Network," Acoustics, Speech, and Signal Processing, ICASSP-90., S15b.5, 1990.

[3] Qinghua Zhang, Albert Benveniste, "Wavelet Networks, IEEE Trans. Neural Network Vol. 3, No. 6, p.889-898, 1992.

[4] Electric Textbook StateSoft, "Activation Function" http://www.statsoft.com/textbook/glosa.html, March 2007.

[5] Wikipedia, "Back Propagation," http://en.wikipedia.org/wiki/Back propagation, March 2007.

[6] Kumpati S. Narendra \& Kannan Parthasarathy, "Identification and Control of Dynamical Systems Using Neural Networks," IEEE Transaction on Neural Networks Vol. 1 No. 1, March 1990

[7] James A. Freeman \& David M. Skapura, "Neural Networks: Algorithms, Application, and Programming Techniques," Addison-Wesley Publishing Company, 1991.

[8] Stuart Russell \& Peter Norvig, "Artificial Intelligence A Modern Approach 2nd Ed.," Prentice Hall 2003.

[9] Simon Haykin, "Neural Networks: A Comprehensive Foundation 2nd Ed.," Prentice Hall 1999.

[10] James Kennedy and Russell C. Eberhart, "particle Swarm Optimization," Proc. IEEE Int. Conf. Neural Networks IV p.1942-1948, 1995.

[11] J. C. Lazaro, J.L. San Emeterio, A. Ramos and J. L. Fernandez-Marron, "Influece of Thresholding Procedures In Ultrasonic Gain Noise Reduction Using Wavelets," 
http://www.sciencedirect.com/science? ob=ArticleURL\& udi=B6TW2-4534HNO-

T\& user $=521828 \& \quad$ rdoc $=1 \& \quad \mathrm{fmt}=\& \quad$ orig $=$ search \& sort=d\&view $=c \&$ acct= $=000059579$

$\underline{\text { \& version }}=1 \&$ urlVersion $=0 \&$ userid $=521828 \& \mathrm{md} 5=\mathrm{fd} 336405 \mathrm{~d} 008 \mathrm{c} 70 \mathrm{bf} 988816 \mathrm{e} 4 \mathrm{c} 5 \mathrm{e} 7$ e31\#toc2, March 2008.

[12] Wikipedia, "Median Absolute Deviation,"

http://en.wikipedia.org/wiki/Median absolute deviation, March 2008.

[13] Wikipedia, "Daubechies Wavelet," http://en.wikipedia.org/wiki/Daubechies_wavelets, March 2008.

[14] Chen, Yuehui, Yang, Bo, Dong, Jiwen, "Time-series Prediction Using a Local Linear Wavelet Neural Network," Neurocomputing 69 p.449-465, 2006.

[15] James A. Freeman \& David M. Skapura, "Neural Networks: Algorithms, Application, and Programming Techniques," Addison-Wesley Publishing Company, 1991.

[16] Addison, Paul S, "The Illustrated Wavelet Transform Handbook, Introductory Theory and Application in science, engineering, Medicine and Finance," Taylor \& Francis Group, 2002.

[17] Ahmad Banakar, "A New Artificial Neural Network and its Application in Wavelet Neural Network and Wavelet Neuro-Fuzzy Case Study: Time Series Prediction," $3^{\text {rd }}$ Inter. IEEE Conf. Intelligent System, p.621-625, 2006.

[18] Chien-Ho Ko, "A Tutorial of Adaptive Fuzzy Inference Systems: fuzzy Logic and Neural Network Based Approaches," http://163.23.28.177/ chko/Web Personal/Tutorial AFIS/Article/Text.htm, October 2008.

[19] No Arthur, "Neuro-Fuzzy and Hybrid Approaches," http://www.stowann.ihe.nl/NFS.htm, October 2008.

[20] Cao Kai, Yoshio Hamamatsu, "An Adaptive Hybrid Wavelet Neural Network and Its Application," Inter. Confer. On Robotics and Biomimetics, p.779-783, 2004.

[21] Dale Dubin, "Rapid Interpretation of EKG's $6^{\text {th }}$ Ed.," COVER Publishing Company, 2000.

[22] Philip J Podrid, "Cardioversion," http://www.uptodate.com/patients/content/topic.do;jsessionid=7E445E401AF7448958 288FEAA6D03239.0503?topicKey= Ac5NyvkIRXhTI\&view=print, October 2008. 
[23] Gary M. Friesen, Thomas C. Jannett, Manal Afify Jadallah, Stanford L. Yates, Stephen R. Quint, H. Troy Nagle, "A Comparison of the Noise Sensitivity of Nine QRS Detection Algorithms," IEEE Trans. on Biomedical Eng. Vol. 37 No. 1 p.85-98, January 1990.

[24] Shen-Tun Li, Shu-Ching Chen, "Function Approximation using Robust Wavelet Neural Networks," $14^{\text {th }}$ IEEE Inter. Confer. on Tools with Artificial Intelligent, p.483-488, 2002.

[25] PhysioBank, "The MIT-BIH Noise Stress Test Database," http://www.physionet.org/physiobank/database/nstdb/, October, 2008.

[26] Azzam-ul-Asar, Syed Riaz ul Hassnain, Affan Khan, "Short Term Load Forecasting Using Particle Swarm Optimization Based ANN Approach," IEEE Inter. Joint Confer. on Neural Network, 2007.

[27] Zachary A. Kissel, "Associative Memory and the Board Game Quarto," http://www.acm.org/crossroads/xrds10-2/autoassoc.html, February, 2009.

[28] Wikipedia, "Wavelet," http://en.wikipedia.org/wiki/Wavelet, February 2008.

[29] Qinghua Zhang, "Using Wavelet Network in Nonparametric Estimation”, IRISA, 1994.

[30] PhysioBank, "MIT-BIH Arrhythmia Database," http://www.physionet.org/physiobank/database/mitdb/, March, 2009.

[31] Yoshifumi Kishimoto, Yasunari Kutsuna, Koji Oguri, "Detecting Motion Artifact ECG Noise During sleeping by Means of a Tri-axis Accelerometer" $29^{\text {th }}$ Annual International Conference of the IEEE EMBS p.2669-2672, August 2007.

[32] Wikipedia, "Electrocardiogram", http://en.wikipedia.org/wiki/ECG, March 2009.

[33] WFDB Applications Guide, "PhysioToolkit", http://www.physionet.org/physiotools/wag/nst-1.htm\#sect4, December 2008.

[34] Wikipedia, "Signal-to-noise ratio," http://en.wikipedia.org/wiki/Signal-tonoise ratio, February 2008.

[35] J. Chen and D. D. Bruns, "WaveARX neural network development for system identification using a systematic design synthesis," Ind. Eng. Chem. Res., vol. 34, p.44204435, 1995

[36] Daniel W. C. Ho, Ping-An Zhang, Jinhua Xu, "Fuzzy Wavelet Networks for Function Learning," IEEE Trans. on Fuzzy Sys. Vol.9 No.1 p.200-211, February 2001. 
[37] Cecil W. Thomas, William P. Huebner, R. John Leigh, "A Low-Pass Notch Filter for Bioelectric Signals," IEEE Transactions on Biomedical Engineering. Vol.35, no.6. p.496498, June 1988.

[38] W. Philips, "Adaptive Noise Removal from Biomedical Signals using Warped Polynomials," Biomedical Engineering, IEEE Transactions on vol. 43, Issue 5, p.480-492, May 1996.

[39] Hiroshi Harada, Hiroshi Kashiwagi, Hikaru Nashimura, "Electromyogram Signal Processing by Using M-transform," Inter Conf on Control Automation and System, p.2446-2449, 2008.

[40] P.Heinonen and Y.Neuvo, "FIR-median hybrid filters," IEEE Transactions on Acoustic Signal and Signal Processing, vol. 35, no. 6, pp. 832-833, 1987.

[41] P.H.Bauer, M. Satori and T.M. Bryden, "An IRR median hybrid filter," IEEE Transactions on Signal Processing, vol. 40, no.5, pp.1068-1078, 1992.

[42] Jui-Hong Horng, "Hybrid MATLAB and LabVIEW with neural network to implement a SCADA system of AC servo motor," Advances in Engineering Software, vol.39 pp.149-155, 2008.

[43] Martin T. Hagan, Howard B. Demuth, Mark Beale, "Neural Network Design," PWS Publishing Company, 1996.

[44] Behzad Mozaffary, Mohammad A. Tinati, "ECG Baseline Wander Elimination using Wavelet Packets," Proceedings of World Academy of Science, Eng. and Tech. Vol. 3 p.1416, January 2005.

[45] C. R. Meyer, H. N. Kelser, "Electrocardiogram baseline noise estimation and removal using cubic splines and state-space computation techniques", Computers and Biomedical Research, vol. 10, pp. 459-470, 1977.

[46] P. W. MacFarlane, J. Peden, J. Lennox, M. P. Watts, T. D. Lawrie, "The Glasgow system", Proc. Of Trends in Computer-Processed Electrocardiograms, North-Holland, pp. 143-150, 1977.

[47] J. A. Van Aleste, T. S. Schilder, "Removal of baseline wander and power-line interference from the ECG by an efficient FIR filter with a reduced number of taps", IEEE Trans. Biomed. Eng., vol BME-32, no. 12, pp. 1052-1060, 1985.

[48] I. I. Christov, I. A. Dotsinsky, I. K. Daskalov, "High pass filtering of ECG signals using QRS elimination", Med. \& Biol. Eng. \& Computing, pp. 253-256, March 1992. 
[49] L. Sornmo, "Time varying digital filtering of ECG baseline wander", Med. \& Biol. Engg. \& Computing, September 1993, pp 503-508.

[50] Paul Kligfield, MD, FAHA, FACC; Leonard S. Gettes, MD, FAHA, FACC; James J. Bailey, MD; Rory Childers, MD; Barbara J. Deal, MD, FACC; E. William Hancock, MD, FACC; Gerard van Herpen, MD, PhD; Jan A. Kors, PhD; Peter Macfarlane, DSc; David M. Mirvis, MD, FAHA; Olle Pahlm, MD, PhD; Pentti Rautaharju, MD, PhD; Galen S. Wagner, MD, "Recommendations for the Standardization and Interpretation of the Electrocardiogram," AHA/ACC/HRS Scientific Statements, Circulation. 2007;115:1306-1324.

[51] American Heart Association's on Electrocardiography, "Recommendations for standardization of lead and specifications for instruments in ECG/VCG", Circulation, 52, pp. 11-25, 1975.

[52] Chavdar Levkov, Georgy Mhov, Ratcho Ivanov, Ivan Daskalov, Ivaylo Christov, Ivan Dotsinsky, "Removal of power-line interference from the ECg: a review of the subtraction procedure," BioMedical Engineering online, 2005.

[53] Chavdar Levkov, Georgy Mihov, Ratch Ivanov, Ivan Daskalov, Ivaylo Christov, Ivan Dotsinsky, "Removal of power-line interference from the ECG: a review of the subtraction procedure," BioMedical Engineering OnLine, 2005.

[54] Pei SC, Tseng CC: Elimination of AC Interference in Electrocardiogram Using IIR Notch Filter with Transient Suppression. IEEE Trans Biomed Eng 1995, 42:1128-1132.

[55] Yoo SK, Kim NH, Song JS, Lee TH, Kim KM: Simple self tuned notch filter in a biopotential amplifier. Med Biol Eng Comput 1997, 35:151-154.

[56] Patrick S. Hamilton, Michael G. Curley, "Adaptive Removal of Motion Artifact," IEEE $19^{\text {th }}$ International conference, p.297-298, 1997.

[57] Tam, H., Webster, J. G., "Minimizing electrode motion artifact by skin abrasion," IEEE Trans. Biomed. Eng., BME-24, pp. 134-139, 1977.

[58] Kahn, A., Greatbatch, W., "Physiologic electrodes," in Medical engineering, Ed. C. D. Ray., Yearbook Medical Publishers, Chicago, 1974.

[59] Burbank D. P., Webster, J. G., "Reducing skin potential motion artifact by skin abrasion," Med. \& Biol. Eng. \& Comput., vol. 16, pp. 31-38, 1978.

[60] Devlin,,P. H., Mark, R. G., Ketchum, J. W., "Detection electrode motion noise in ECG signals by monitoring electrode impedance," Computers in Cardiology, pp. 51 56, 1984. 
[61] M. Milanesi, N. Martini, N. Vanello, V. Positano, M. F. Santarelli, R. Paradiso, D. De Rossi, L. Landini, "Multichannel Techniques for Motion Artifacts Removal from Electrocardiographic Signals," $28^{\text {th }}$ IEE EMBS Annual Inter Conference, p.3391-3394, 2006.

[62] D. P. Burbank, J. G. Webster, "Reducing skin potential motion artifacts by skin abrasion," Med. Biol. Eng. Comput., vol. 16, pp. 31-38, 1978

[63] J. G. Webster, "Reducing motion artifacts and interference in biopotential recording," IEEE Trans. Biomed. Eng., vol. BME-31, pp. 823-826, December 1984.

[64] Bassam Rhou, Mohamad Sawan, Tommy Desilets, Francois Bellemare, "Real-time filtering technique to remove ECG interference from recorded esophageal EMG," Biomedical Circuits and Systems Conference, November 2008

[65] Alex Aussem, Fionn Murtagh, "Combining Neural Network Forecasts on WaveletTransformed Time Series," Connection Science, 1996

[66] Kok Keong Teo, Lipo Wang, Zhiping Lin, "Wavelet Packet Multi-layer Perceptron for Chaotic Time Series Prediction: Effects of Weight Initialization," LNCS, pp.310-317, 2001

[67] Rodrigo Varejao Andreao, Bernadette Dorizzi, Paulo Cesar Cortez, Joao Cesar Moura Mota, "Efficient ECG multi-level wavelet classification through neural network dimensionality reduction," IEEE, 2002

[68] Xiao-Ping Zhang, M.. Desai, "Nonlinear Adaptive Noise Suppression Based On Wavelet Transform," Proc. Of ICASSP, May 1998 


\section{APPENDICES}

\section{Noise Reduction via Wavelet Decomposition and Neural Network}

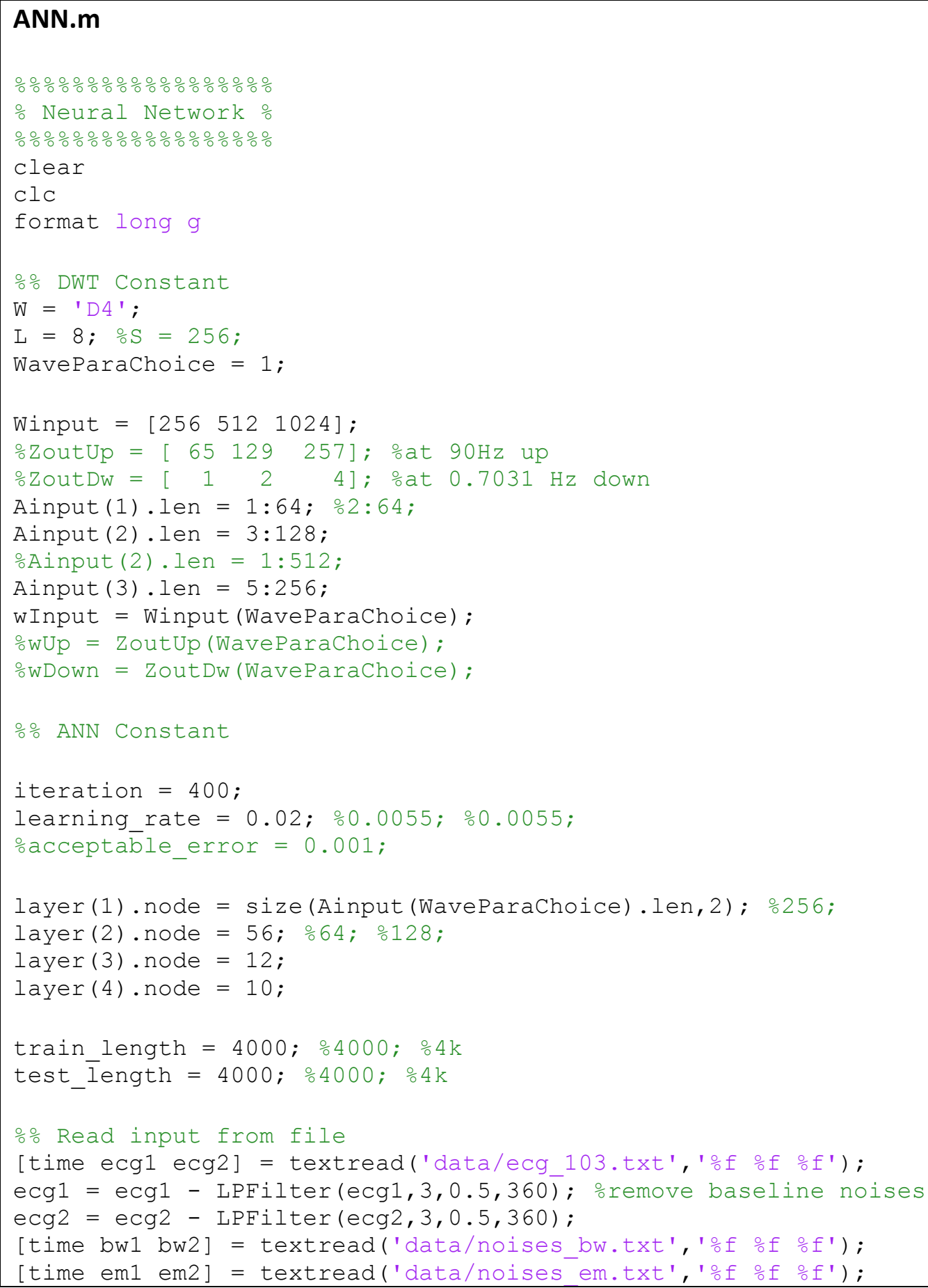




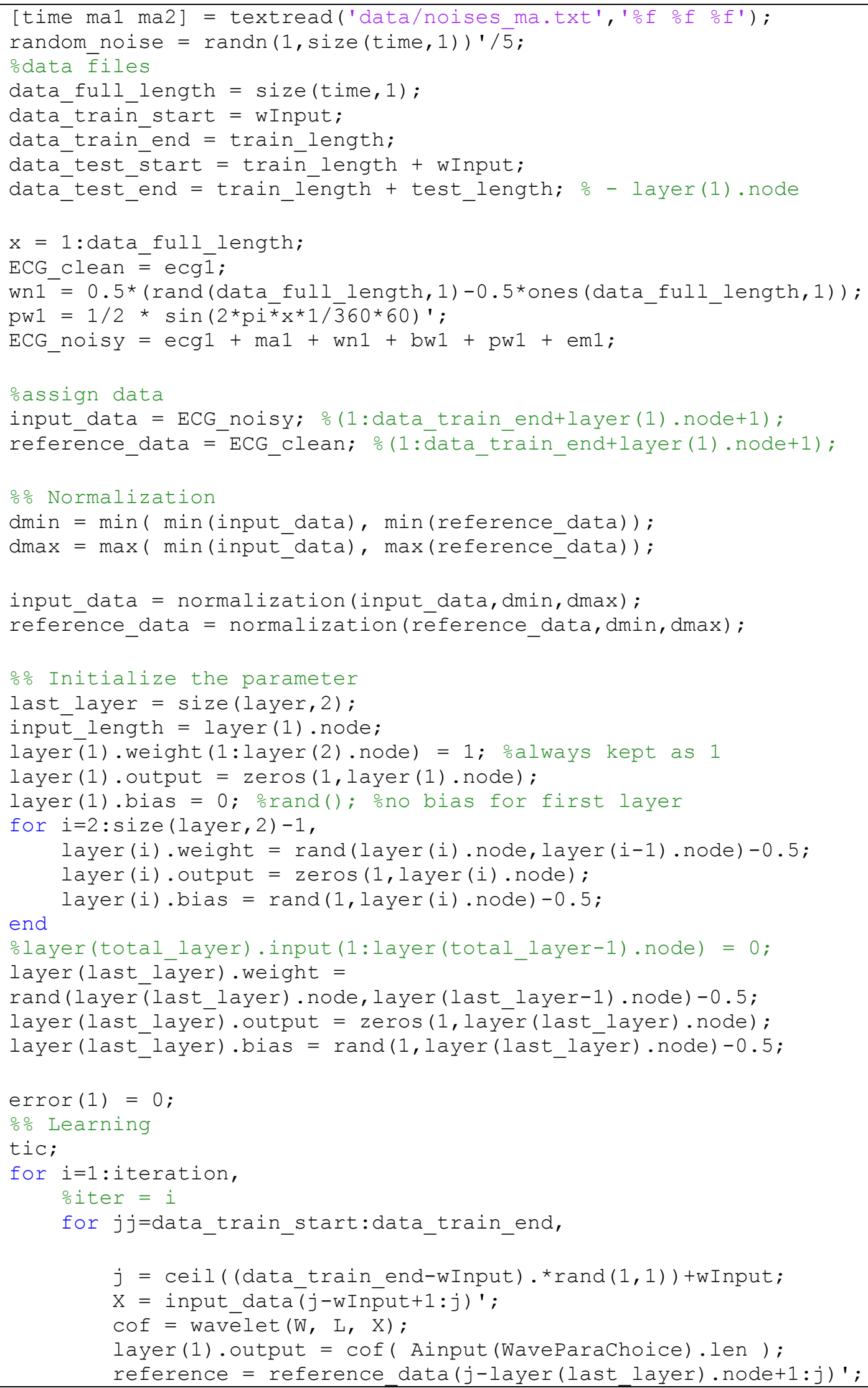




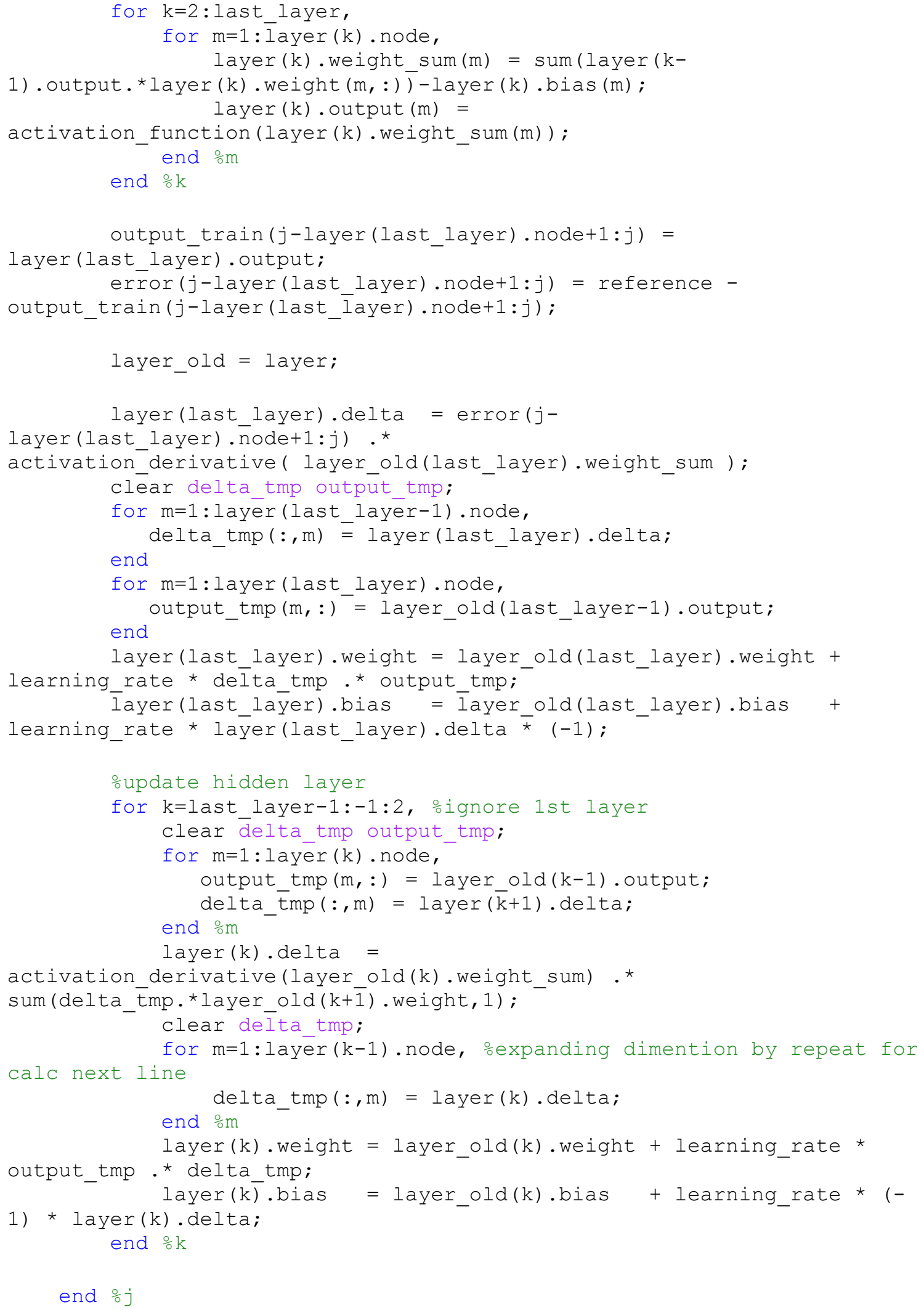




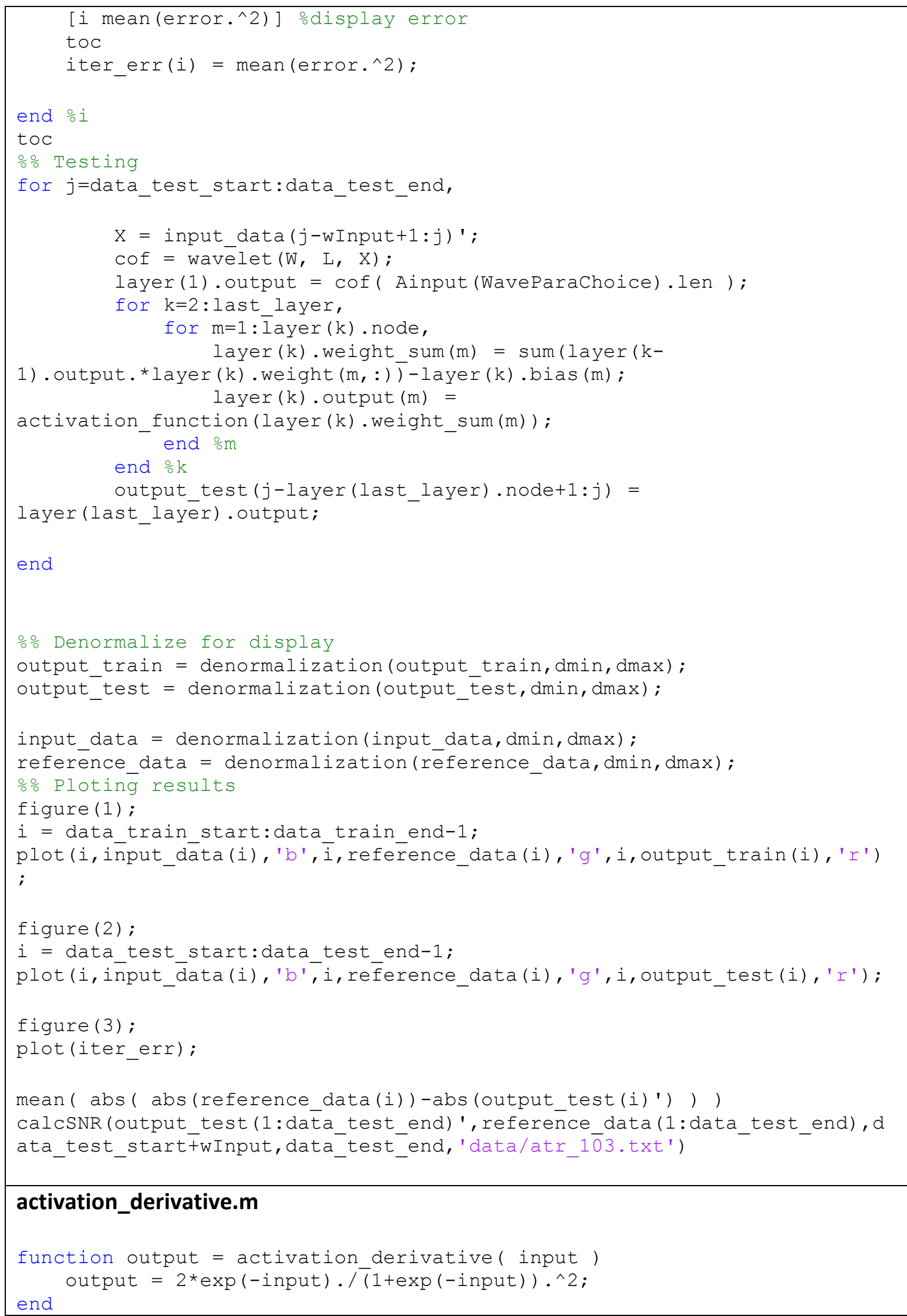




\section{Activation_function.m}

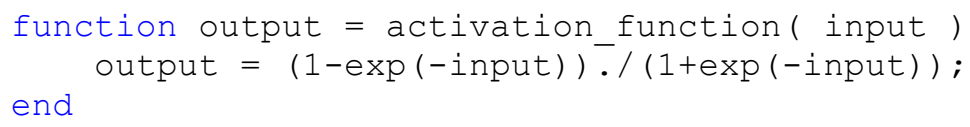

\section{calcSNR.m}

function snr = calcSNR( noisy record, clean record, index start, index end, annotator file)

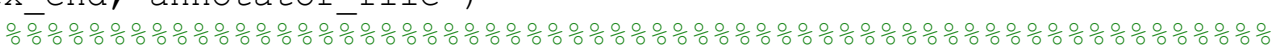

\% Mehod: http://www.physionet.org/physiotools/wag/nst-1.htm \%

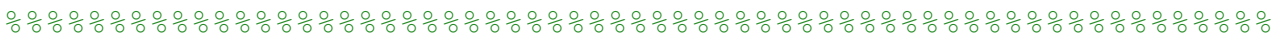

[time atr_sample xtype xsub xchan xnum] =

textread (annotator file, '응

o+1 because annotator read from index 0

atr_sample = atr_sample + 1;

ostrcat $(a, b)$

noise record = noisy record - clean record;

window before $=16$; $\frac{\text { o }}{0}$ bout $50 \mathrm{~ms}$

window_after $=32 ; \circ 16-32$ because just to ensure that $\mathrm{S}$ wave is covered

noise chunk $=360$; $\frac{\circ}{c}$ alc 1 second chunks at a time

ofind noisy signal power

ostart index search in annotator file

count $=1$;

ptop(count) $=0$; oinitialize to zero

for $i=2$ :size(atr_sample,1), ostart at 2 because 1 is (+ Rhythm change)

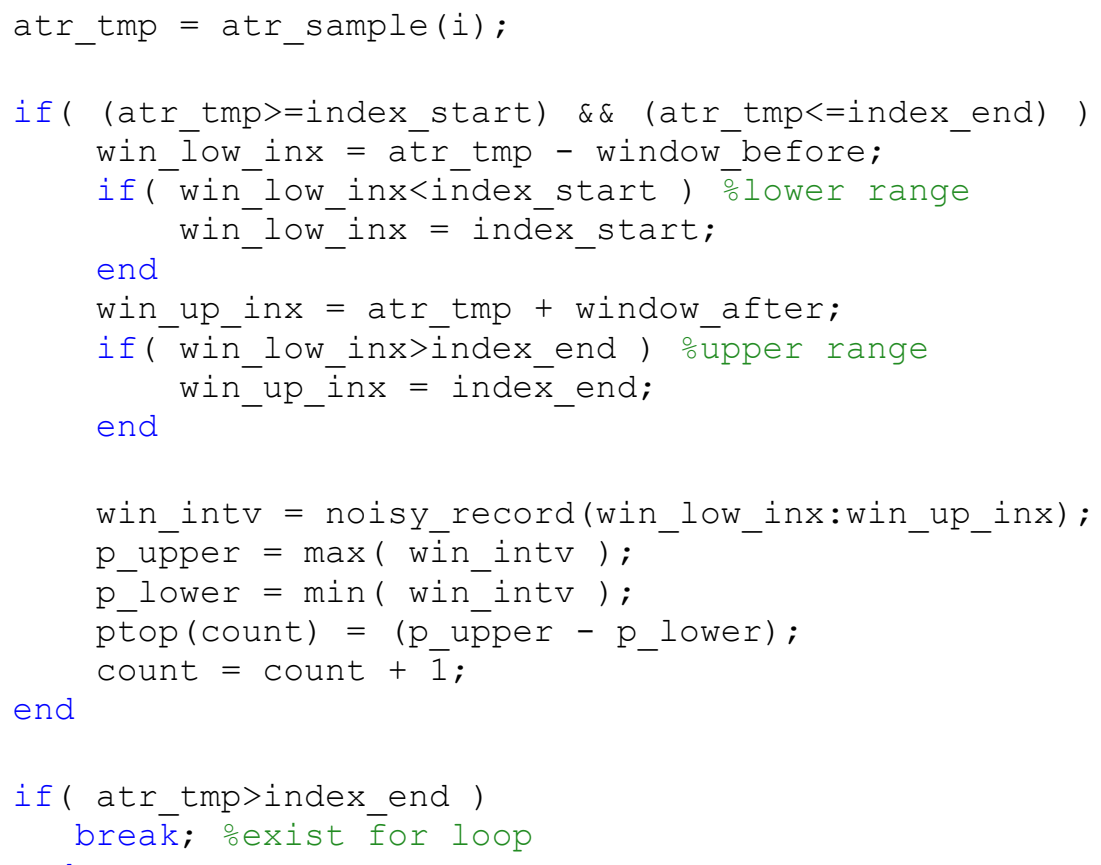




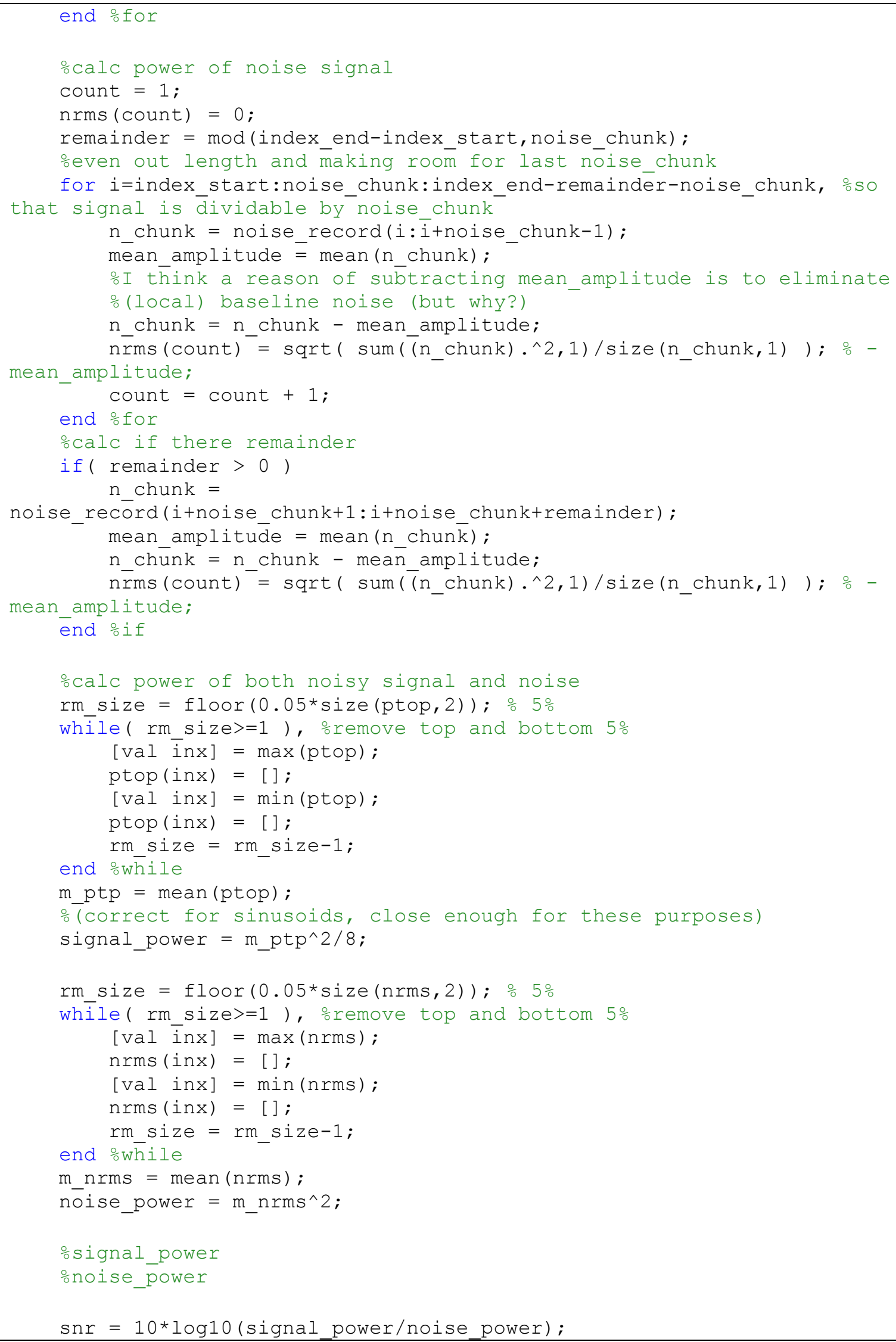




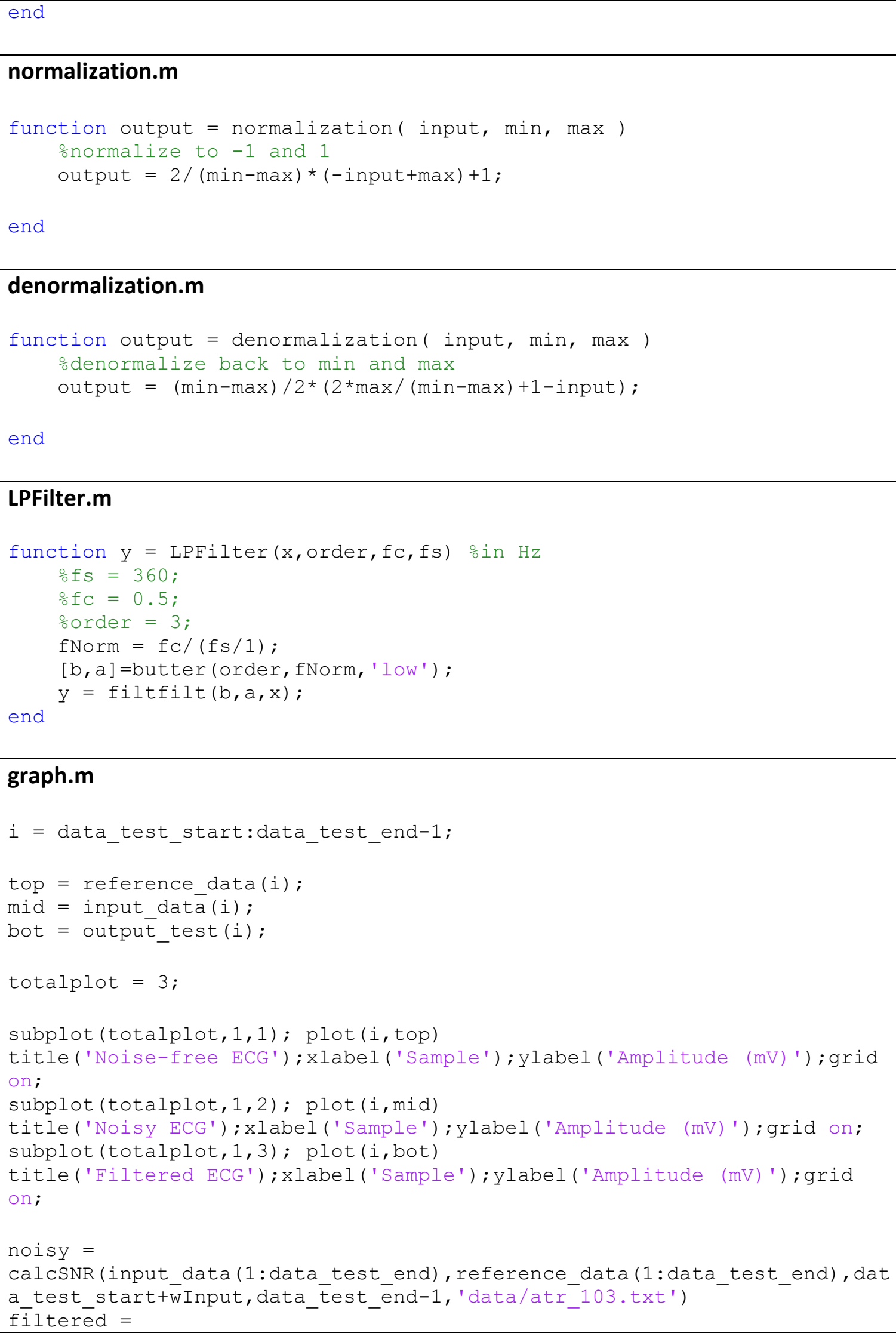

\section{LPFilter.m}

graph.m

$i=$ data_test_start:data_test_end-1; 
calcSNR (output_test (1:data_test_end) ', reference_data (1:data_test_end), d ata_test_start+̄oInput, data_test_end-1, 'data/atr_103.txt')

\section{wavelet.m}

function varargout $=$ wavelet (WaveletName, Level, X, Ext, Dim)

\%WAVELET Discrete wavelet transform.

$\div \quad Y=$ WAVELET $(W, L, X)$ computes the L-stage discrete wavelet transform (DWT) of signal $X$ using wavelet $W$. The length of $X$ must be divisible by $2^{\wedge} \mathrm{L}$. For the inverse transform, $\operatorname{WAVELET}(W,-\mathrm{L}, \mathrm{X})$ inverts L stages. Choices for $W$ are

'Haar'

Haar

'D1', 'D2', 'D3', 'D4', 'D5', 'D6'

Daubechies'

'Sym1', 'Sym2', 'Sym3 ', 'Sym4', 'Sym5 ', 'Sym6'

Symlets

'Coif1', 'Coif2'

'BCoif1'

'Spline Nr.Nd' (or 'bior Nr.Nd') for

$\mathrm{Nr}=0, \mathrm{Nd}=0,1,2,3,4,5,6,7$, or 8

$\mathrm{Nr}=1, \mathrm{Nd}=0,1,3,5$, or 7

$\mathrm{Nr}=2, \mathrm{Nd}=0,1,2,4,6$, or 8

$\mathrm{Nr}=3, \mathrm{Nd}=0,1,3,5$, or 7

$\mathrm{Nr}=4, \mathrm{Nd}=0,1,2,4,6$, or 8

$\mathrm{Nr}=5, \mathrm{Nd}=0,1,3$, or 5

'RSpline Nr.Nd' for the same Nr.Nd pairs Reverse splines

' $\mathrm{S}+\mathrm{P}(2,2)$ ', 'S+P $(4,2)$ ', ' $\mathrm{S}+\mathrm{P}(6,2)$ ',

'S+P $(4,4)^{\prime}, ' S+P(2+2,2)$ '

' $\mathrm{TT}$ '

Coiflets

Coiflet-like [2]

'LC 5/3','LC 2/6', 'LC 9/7-M', 'LC 2/10',

splines

'LC 5/11-C','LC 5/11-A','LC 6/14',

'LC 13/7-T', 'LC 13/7-C'

'Le Gall 5/3','CDF 9/7'

' $\mathrm{V} 9 / 3$ '

' Lazy'

$\mathrm{S}+\mathrm{P}$ wavelets [3]

"Two-Ten" [5]

Low Complexity [1]

Case and spaces are ignored in wavelet names, for example, 'Sym4' may also be written as 'sym 4'. Some wavelets have multiple names, 'D1', 'Sym1', and 'Spline 1.1' are aliases of the Haar wavelet.

WAVELET $(W)$ displays information about wavelet $W$ and plots the primal and dual scaling and wavelet functions.

For 2D transforms, prefix W with '2D'. For example, '2D S+P $(2,2)$ ' specifies a 2D (tensor) transform with the $\mathrm{S}+\mathrm{P}(2,2)$ wavelet. 2D transforms require that $\mathrm{X}$ is either $\mathrm{MxN}$ or $\mathrm{MxNxP}$ where $\mathrm{M}$ and $\mathrm{N}$ are divisible by $2^{\wedge} \mathrm{L}$.

WAVELET (W, L, X,EXT) specifies boundary handling EXT. Choices are

'sym' Symmetric extension (same as 'wsw')

'asym' Antisymmetric extension, whole-point antisymmetry

'zpd' Zero-padding

'per' Periodic extension

'spo' Constant extrapolation

Various symmetric extensions are supported:

'WSWS' Whole-point symmetry (WS) on both boundaries

'hshs' Half-point symmetry (HS) on both boundaries

'wshs' WS left boundary, HS right boundary 


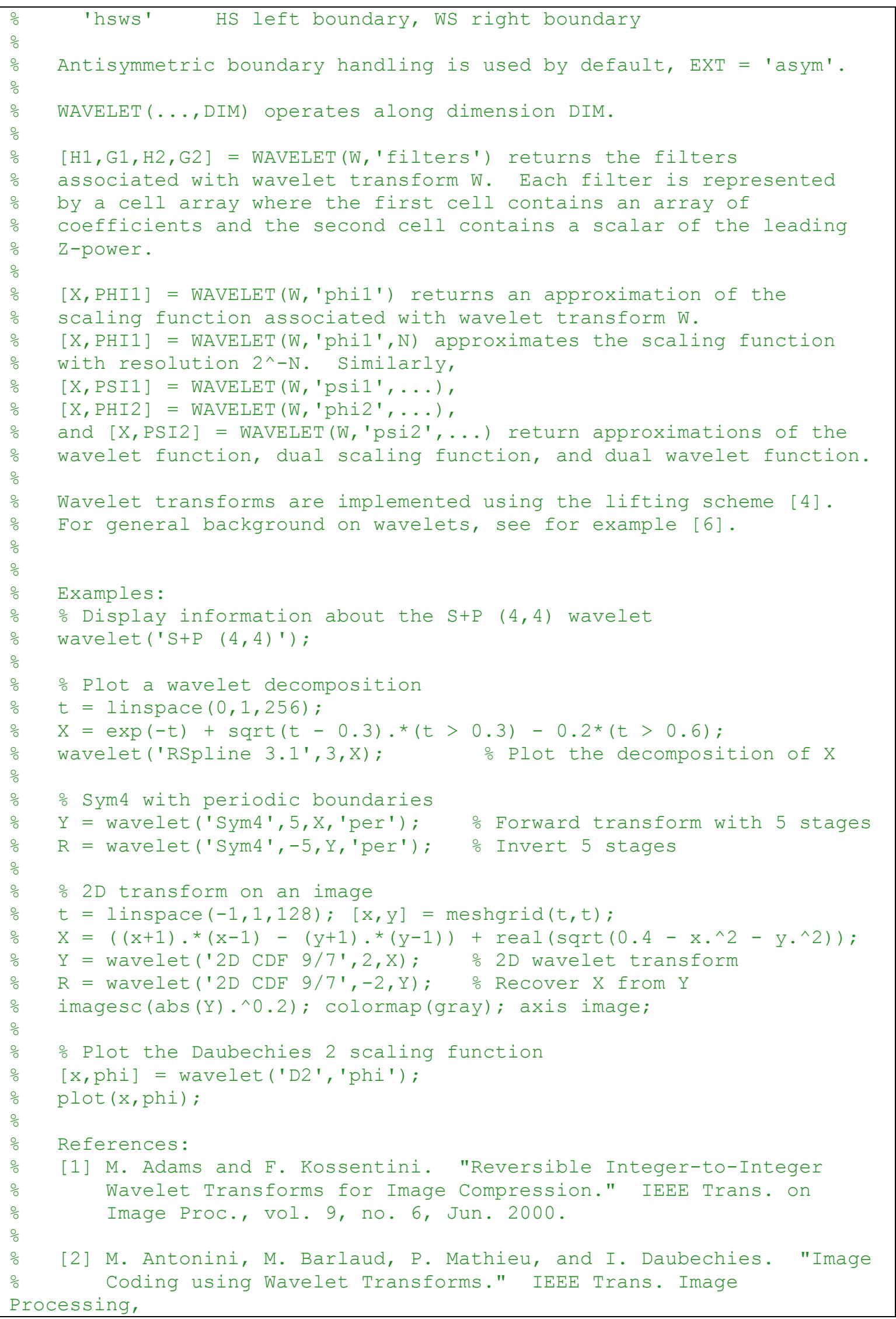




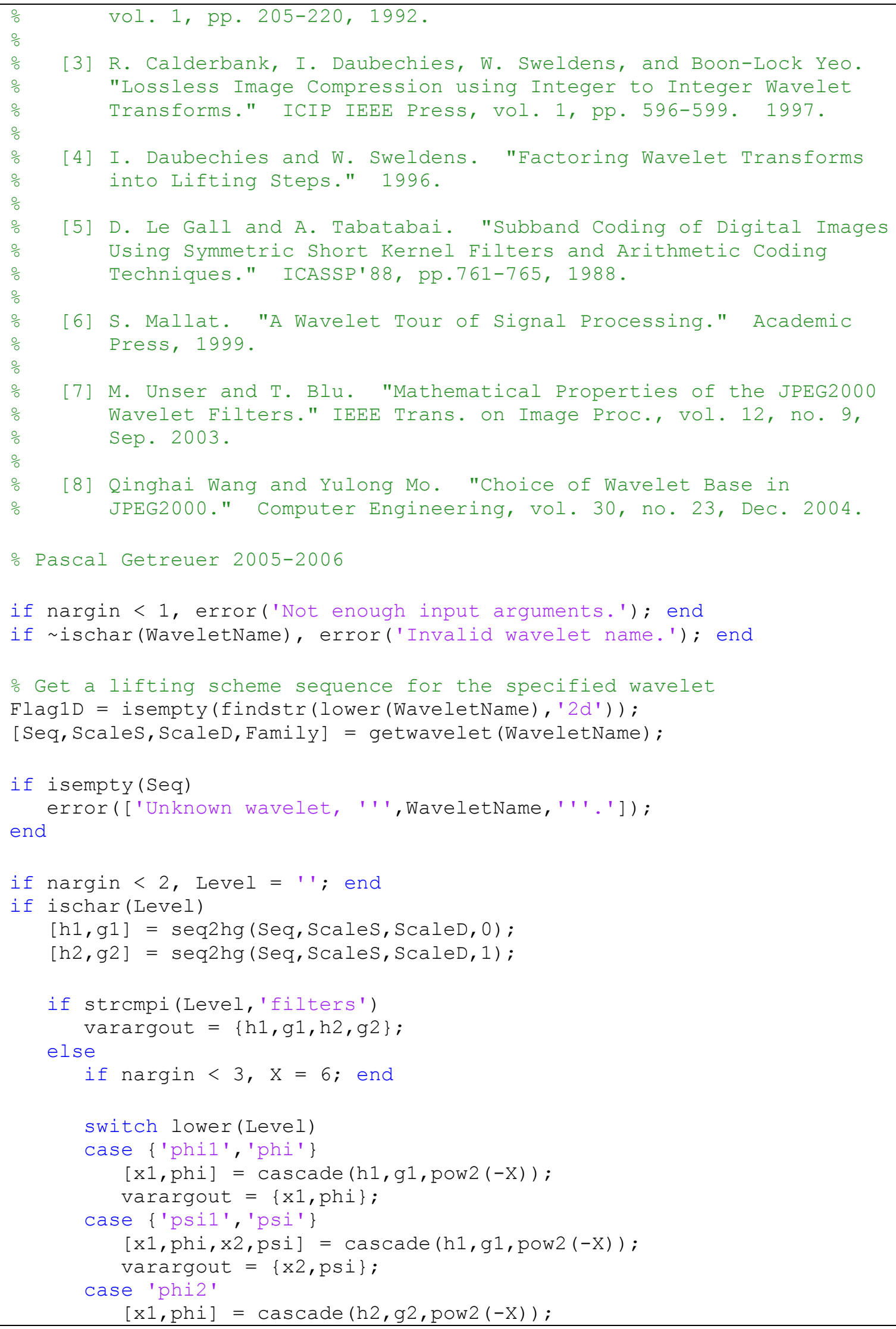




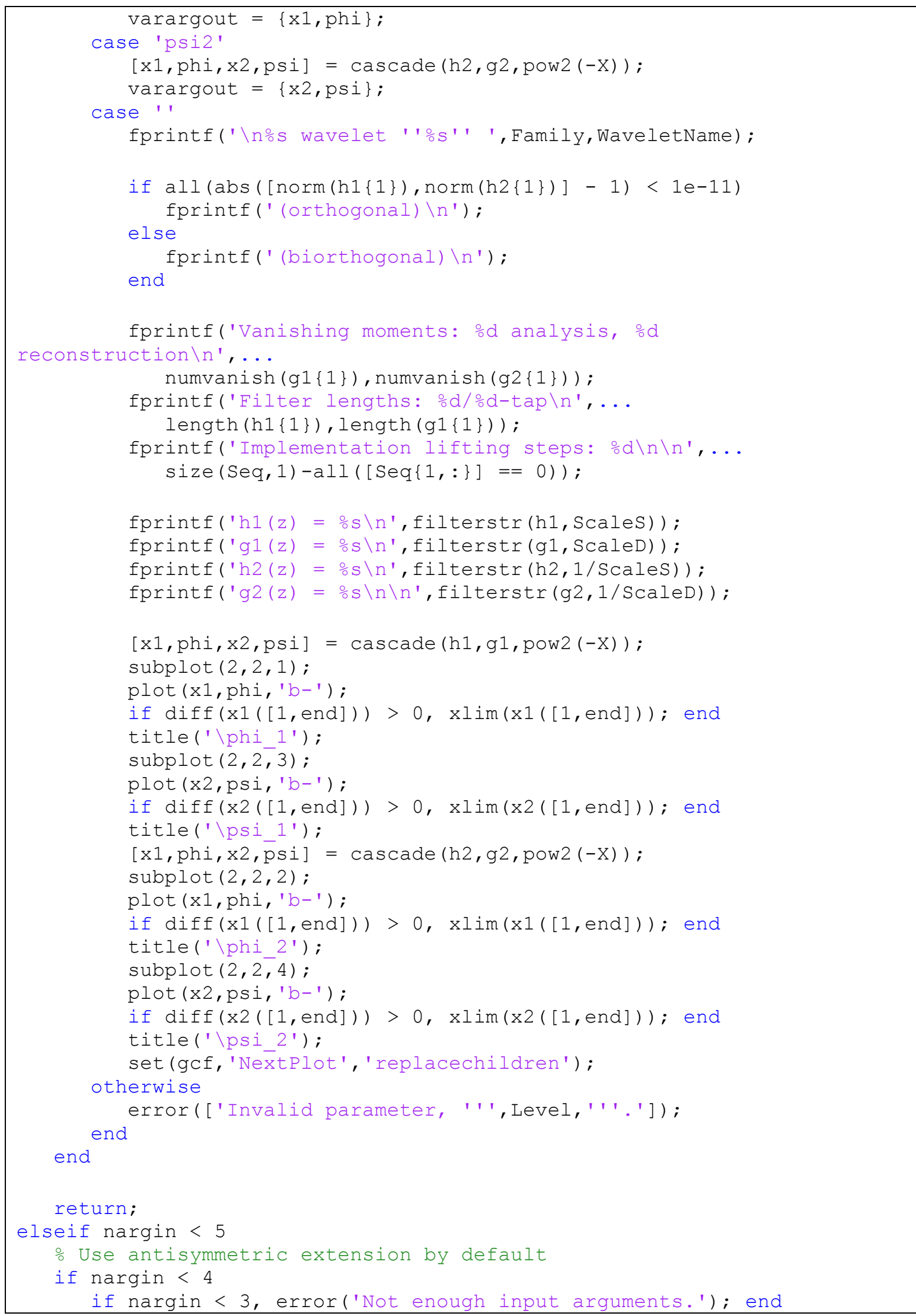




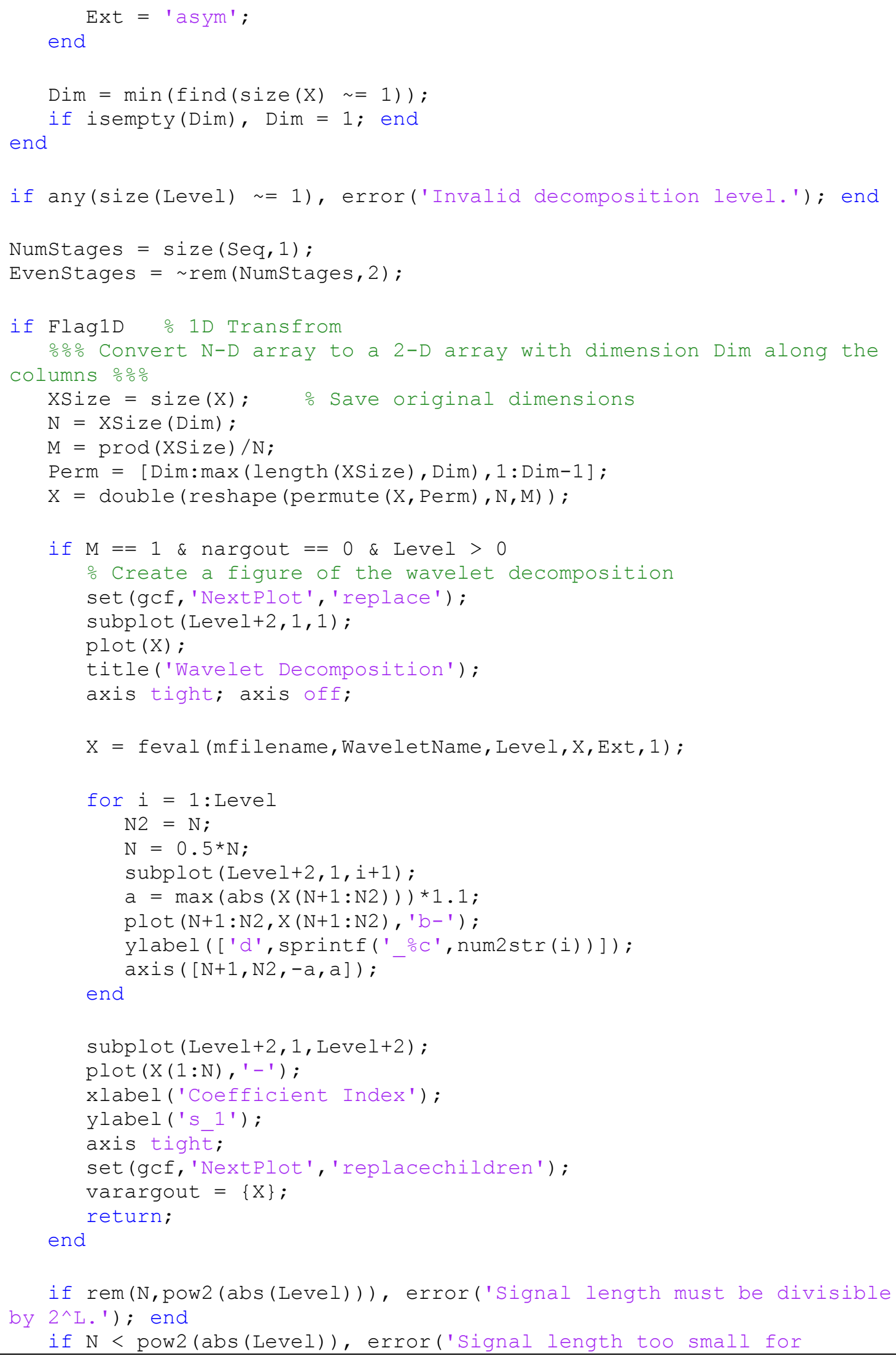




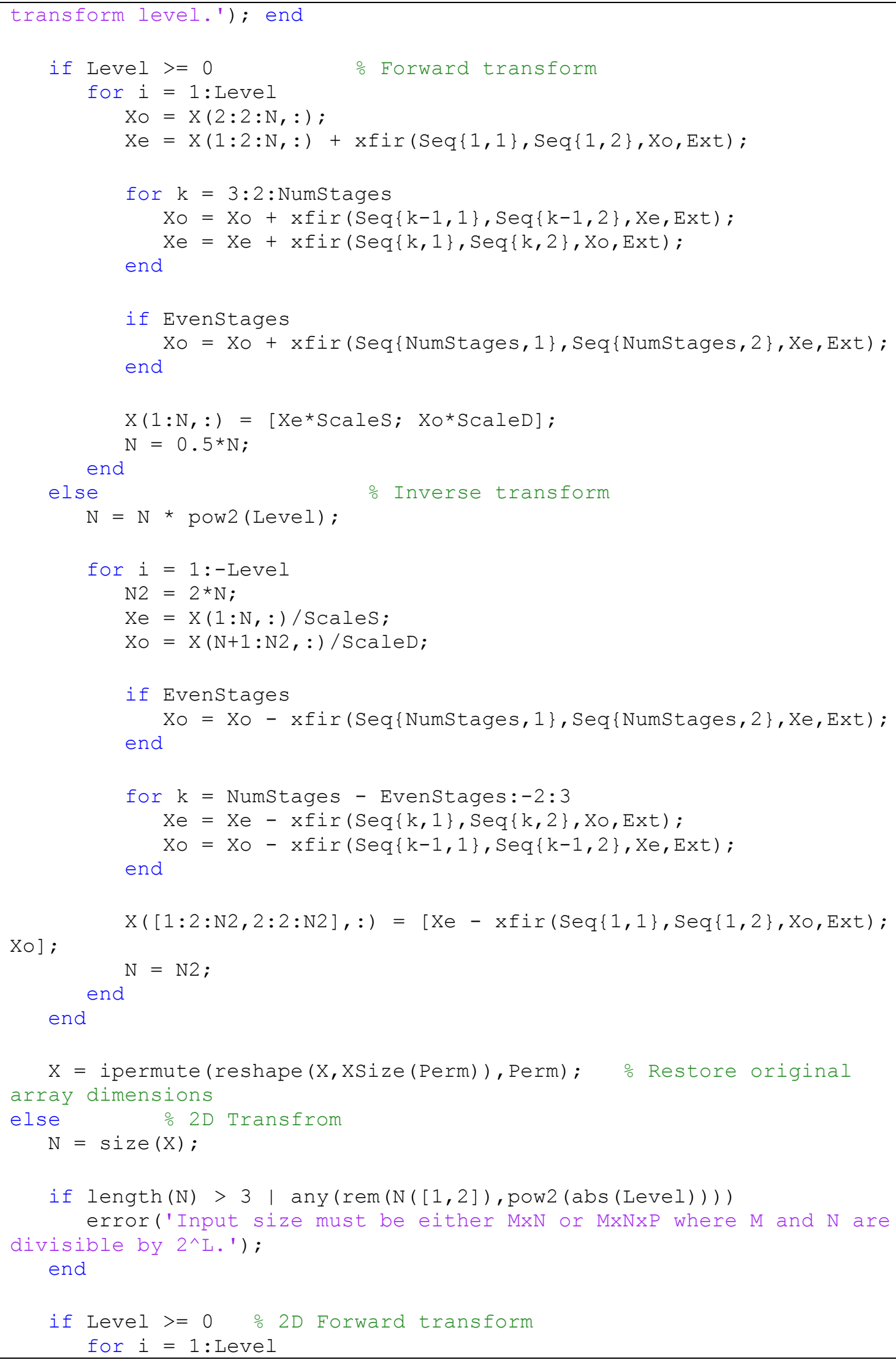




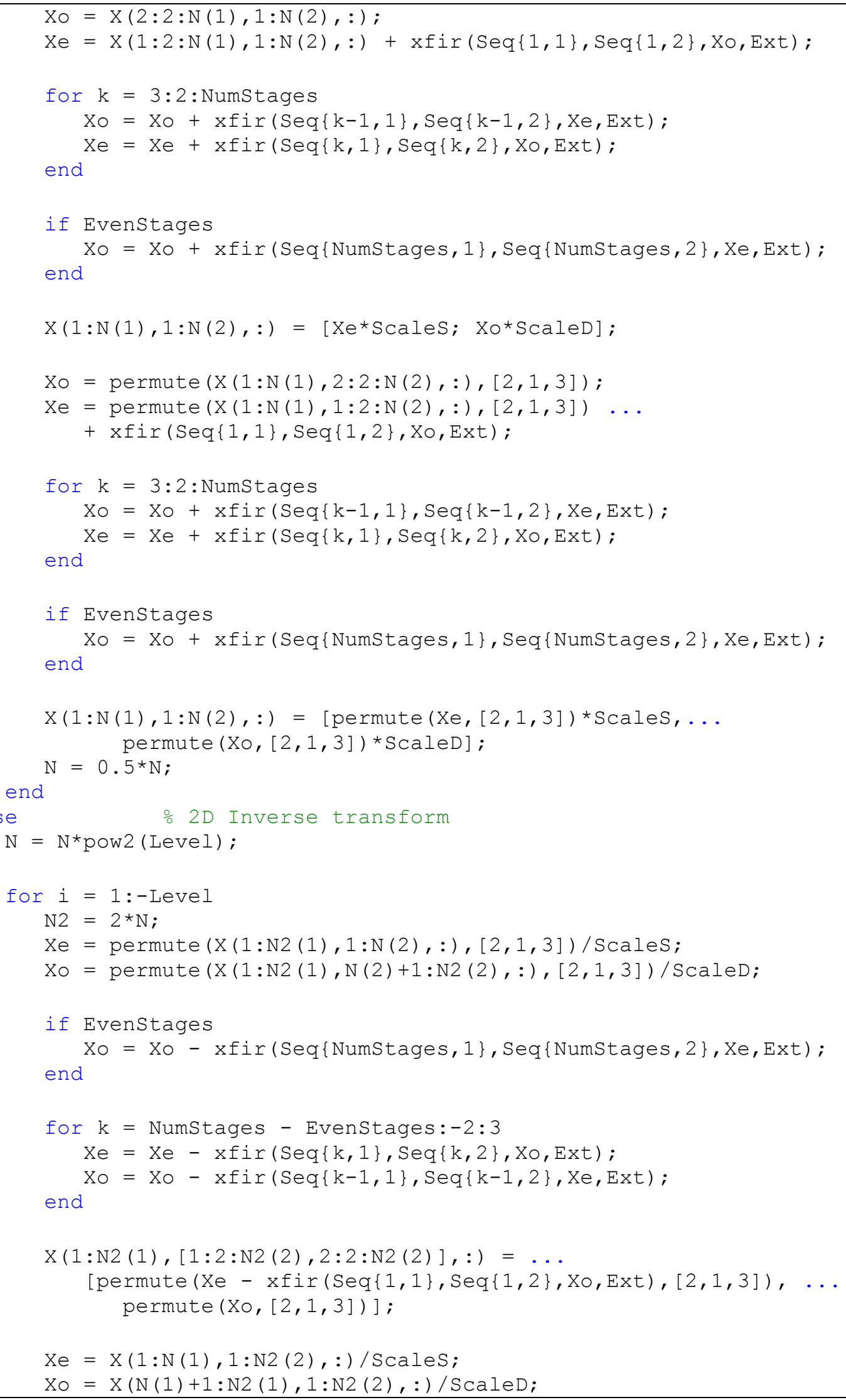




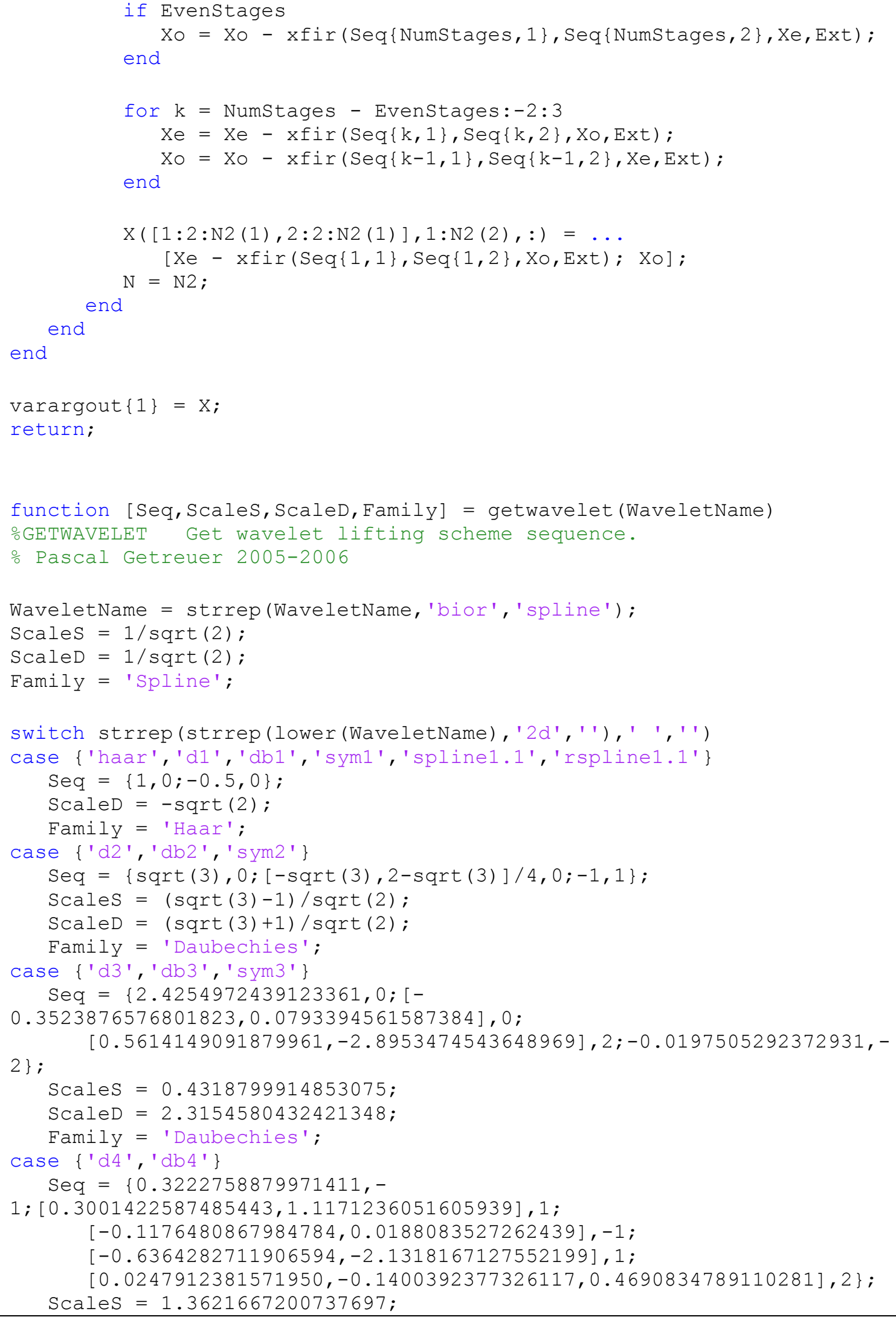




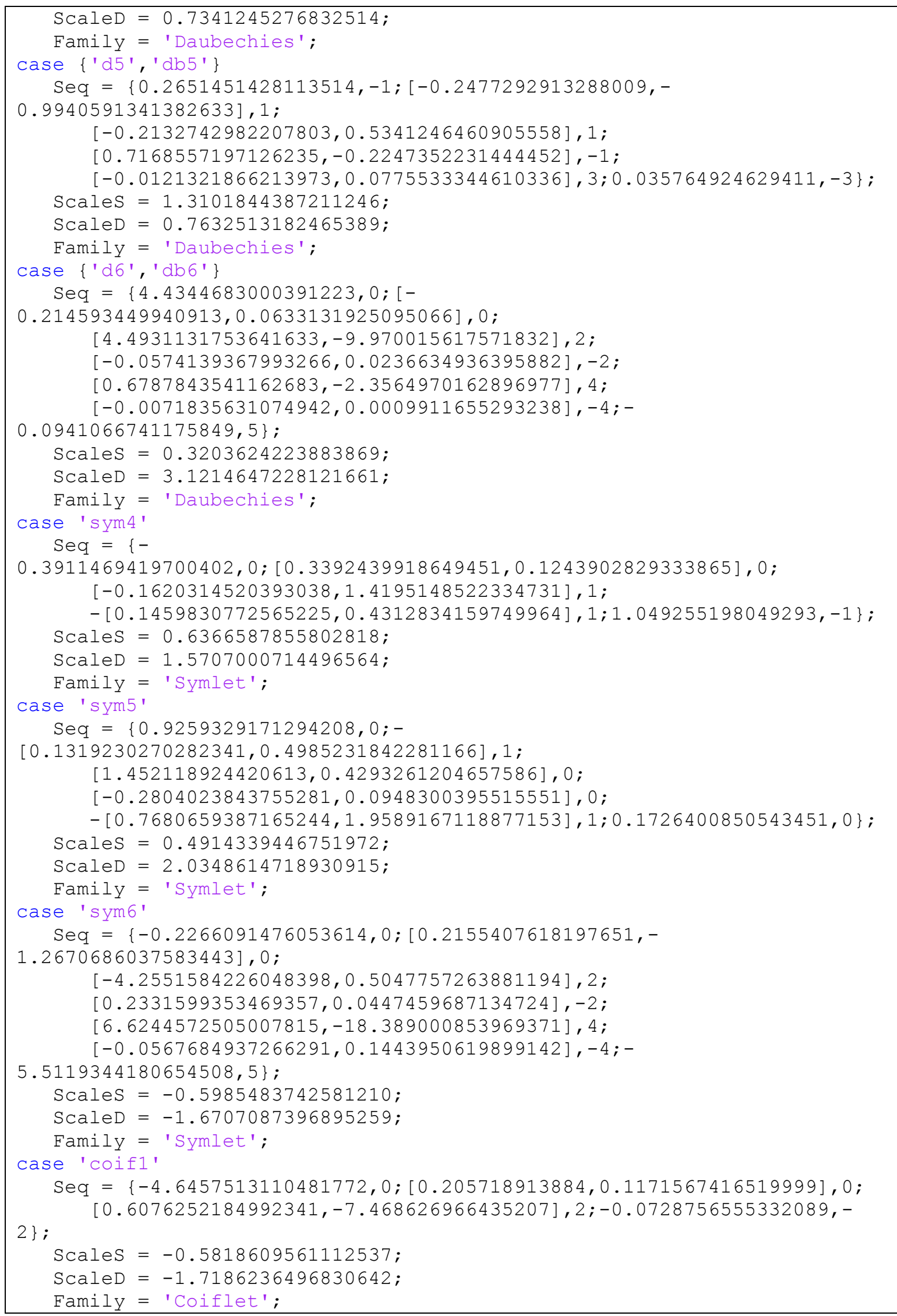




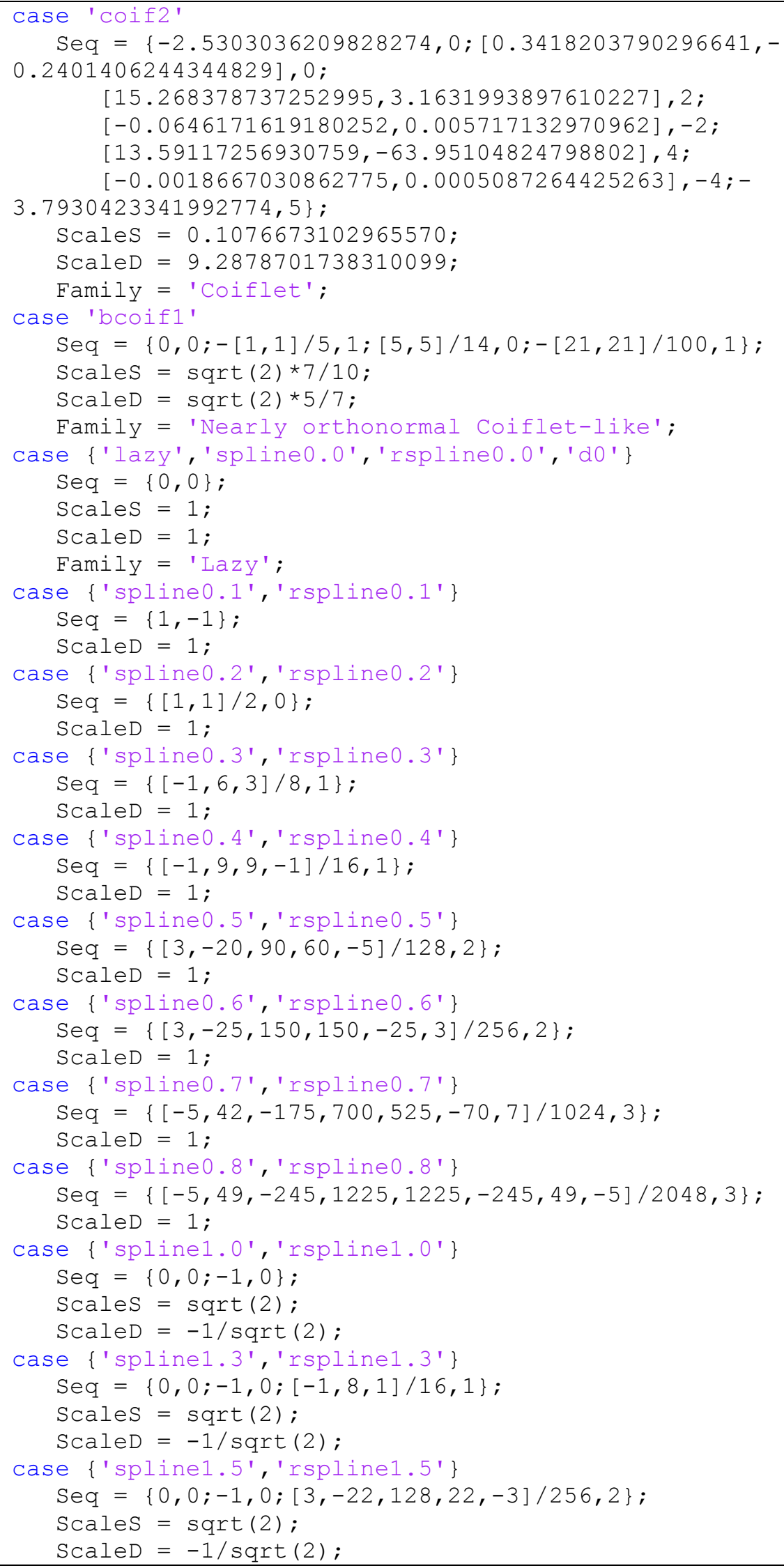




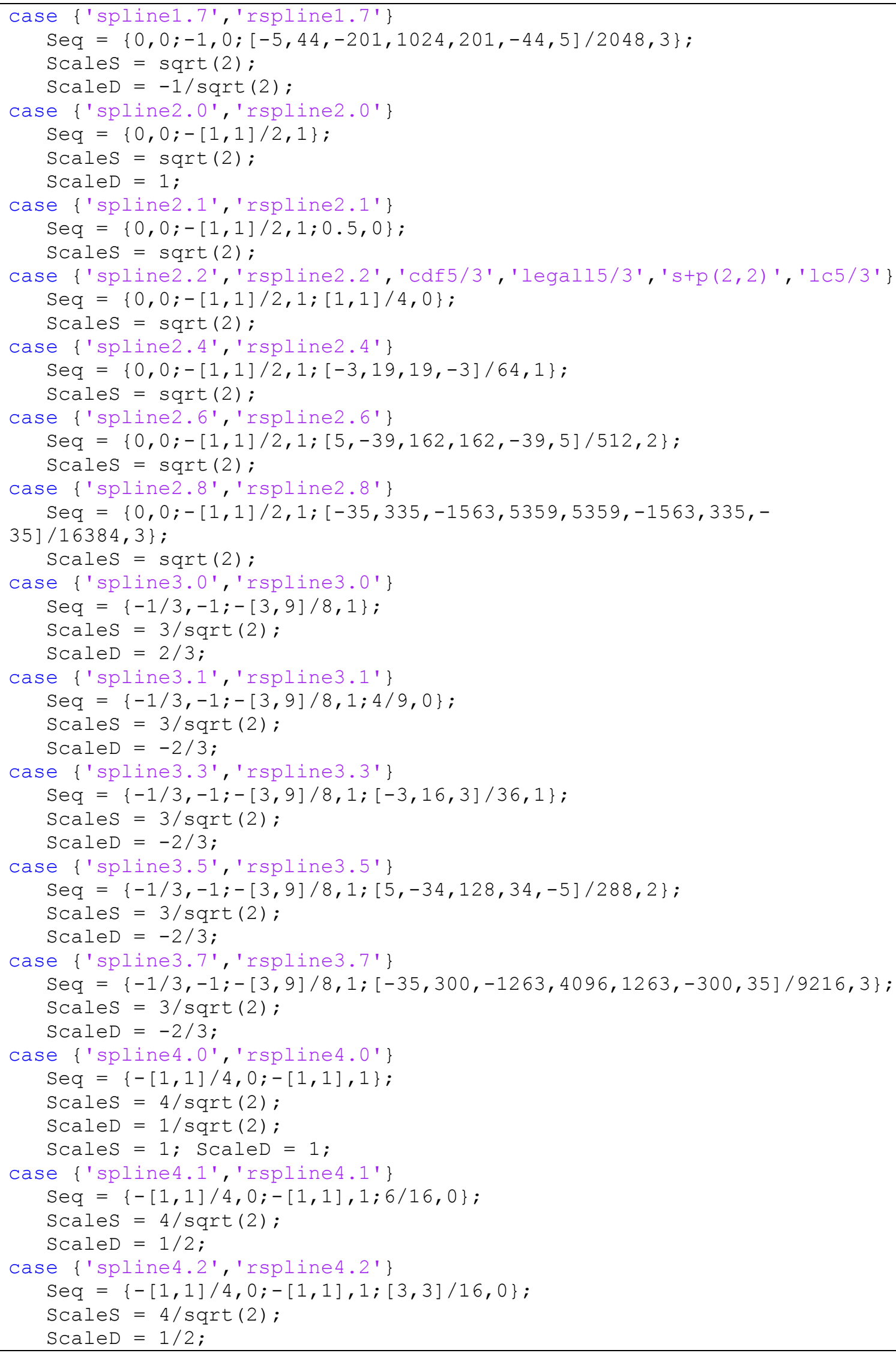




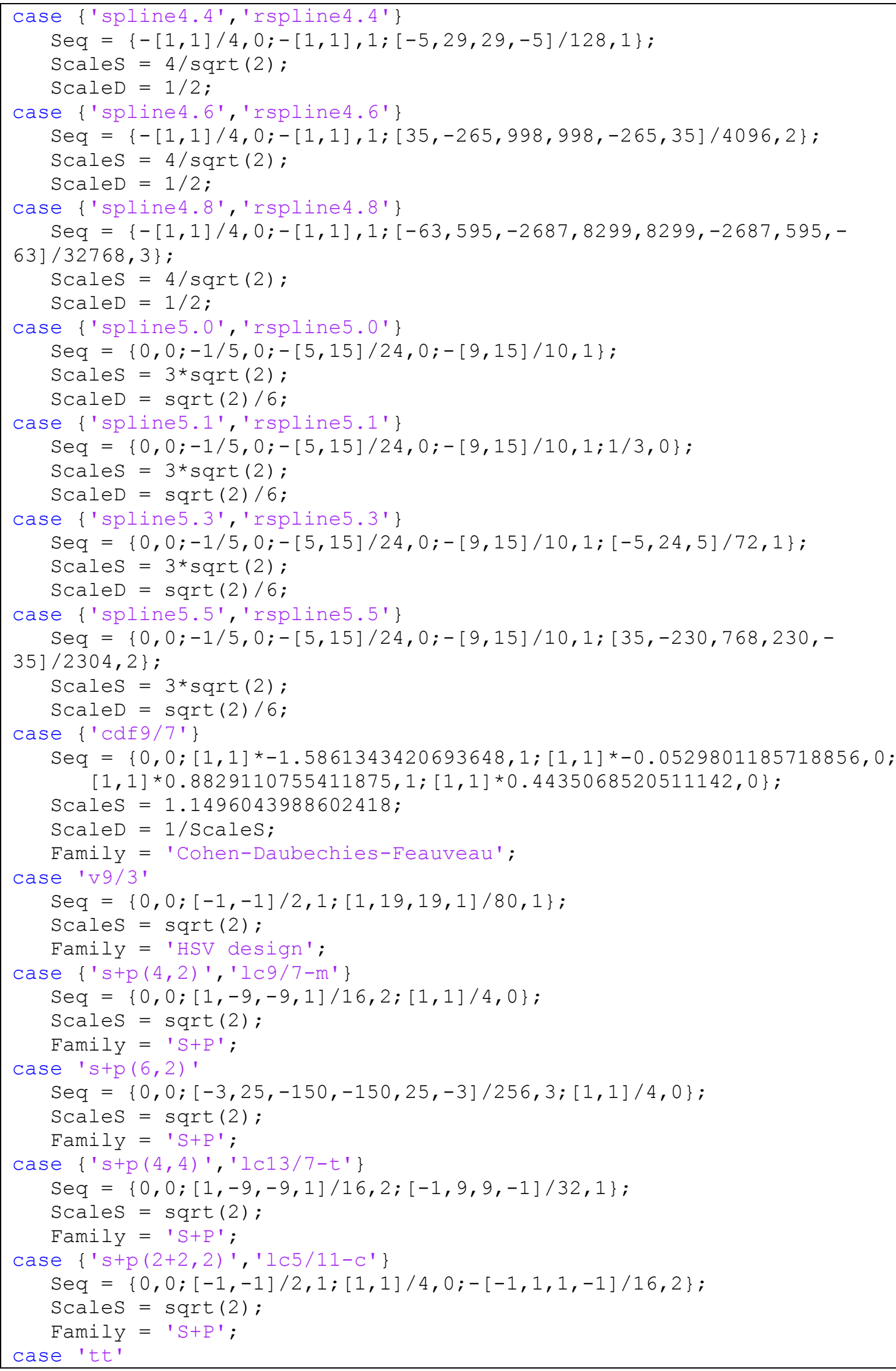




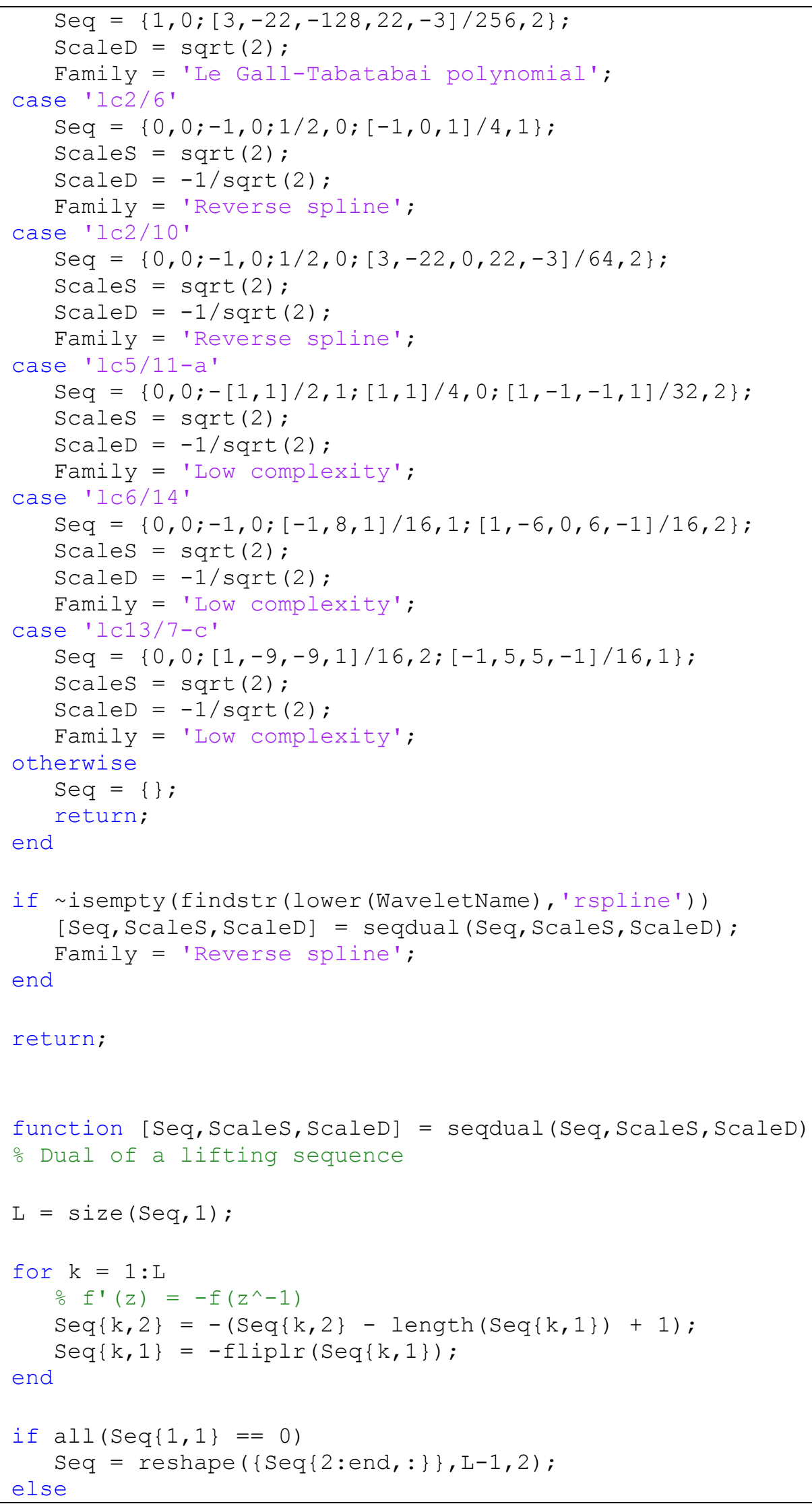




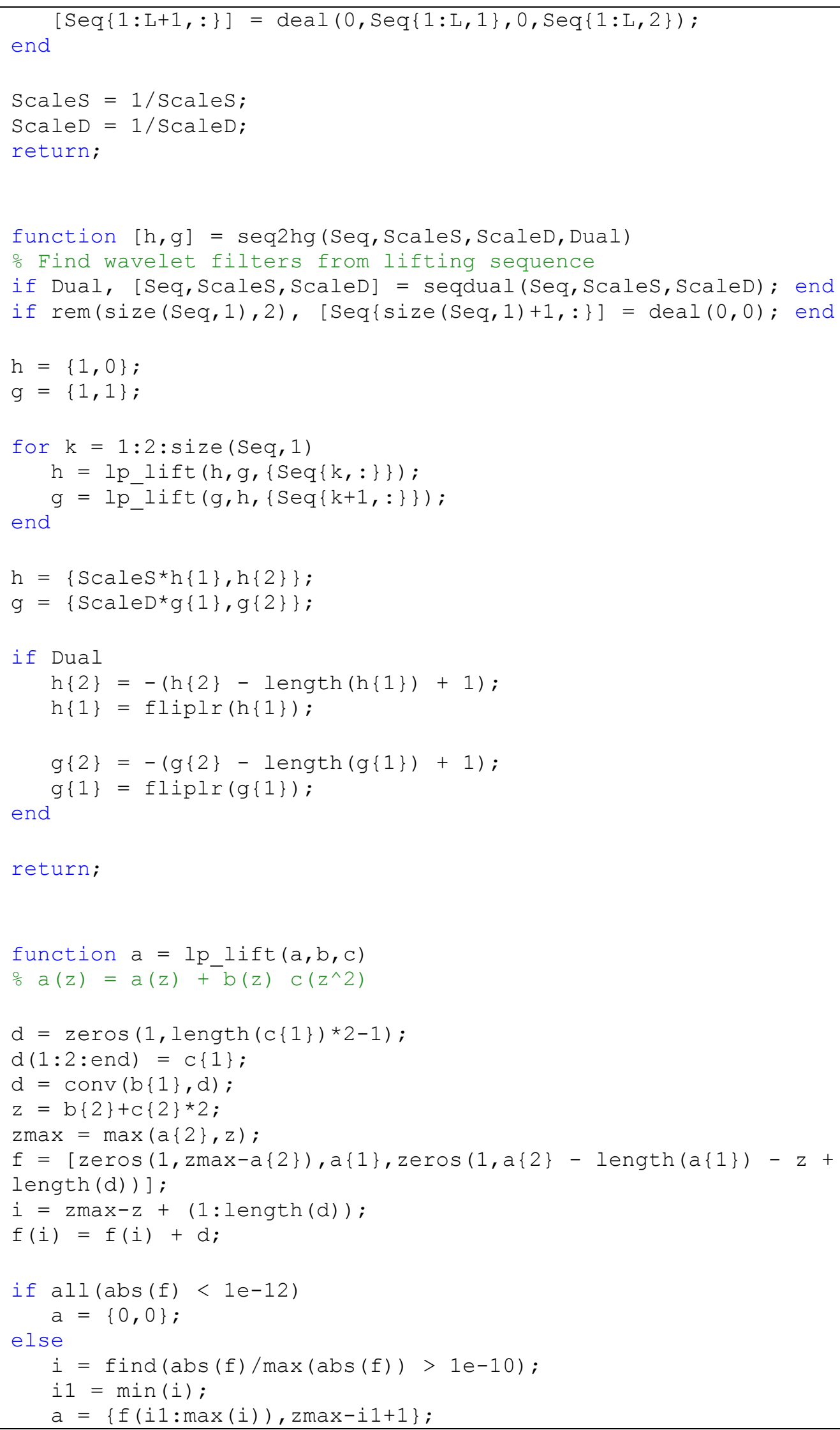




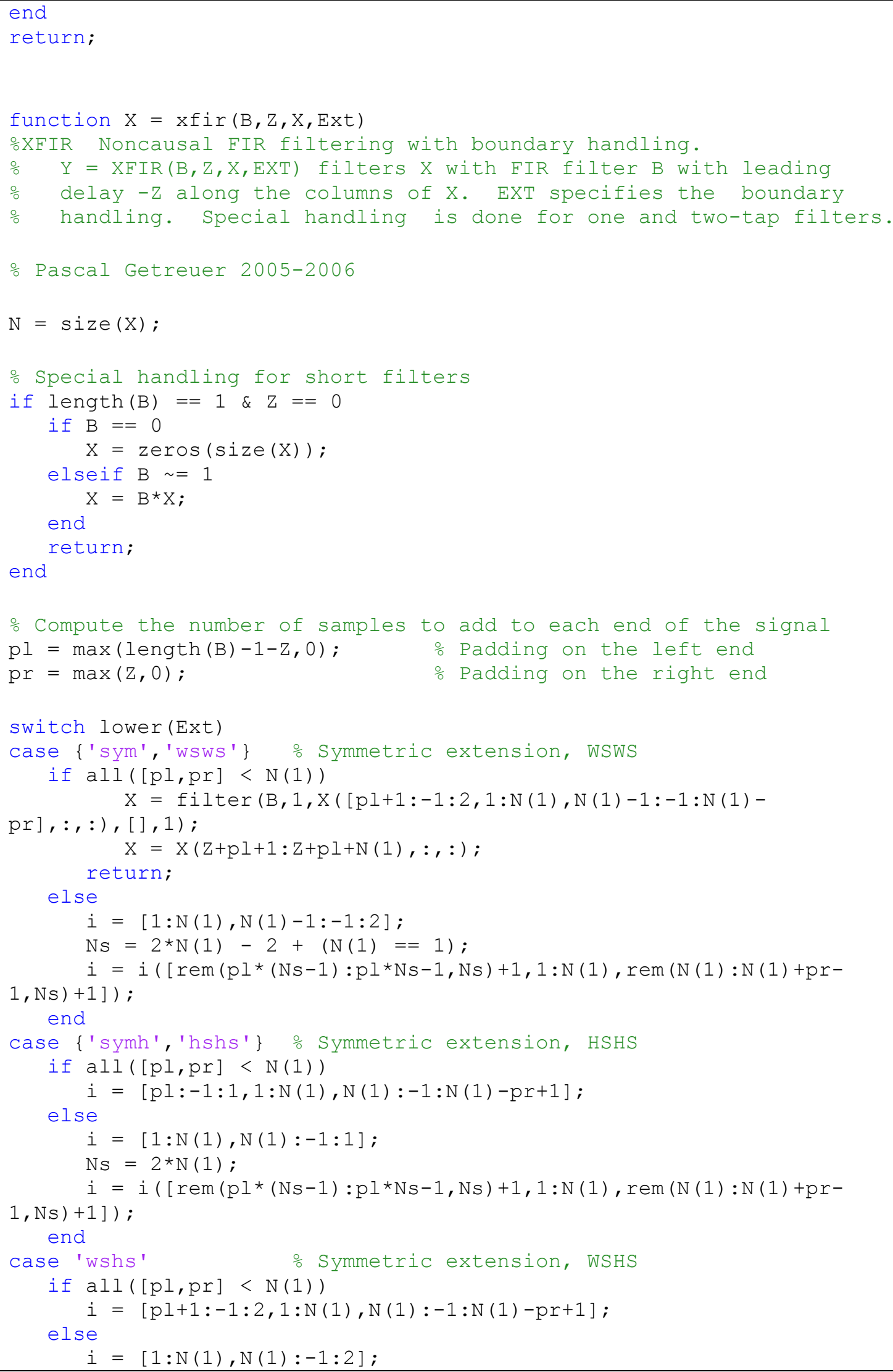




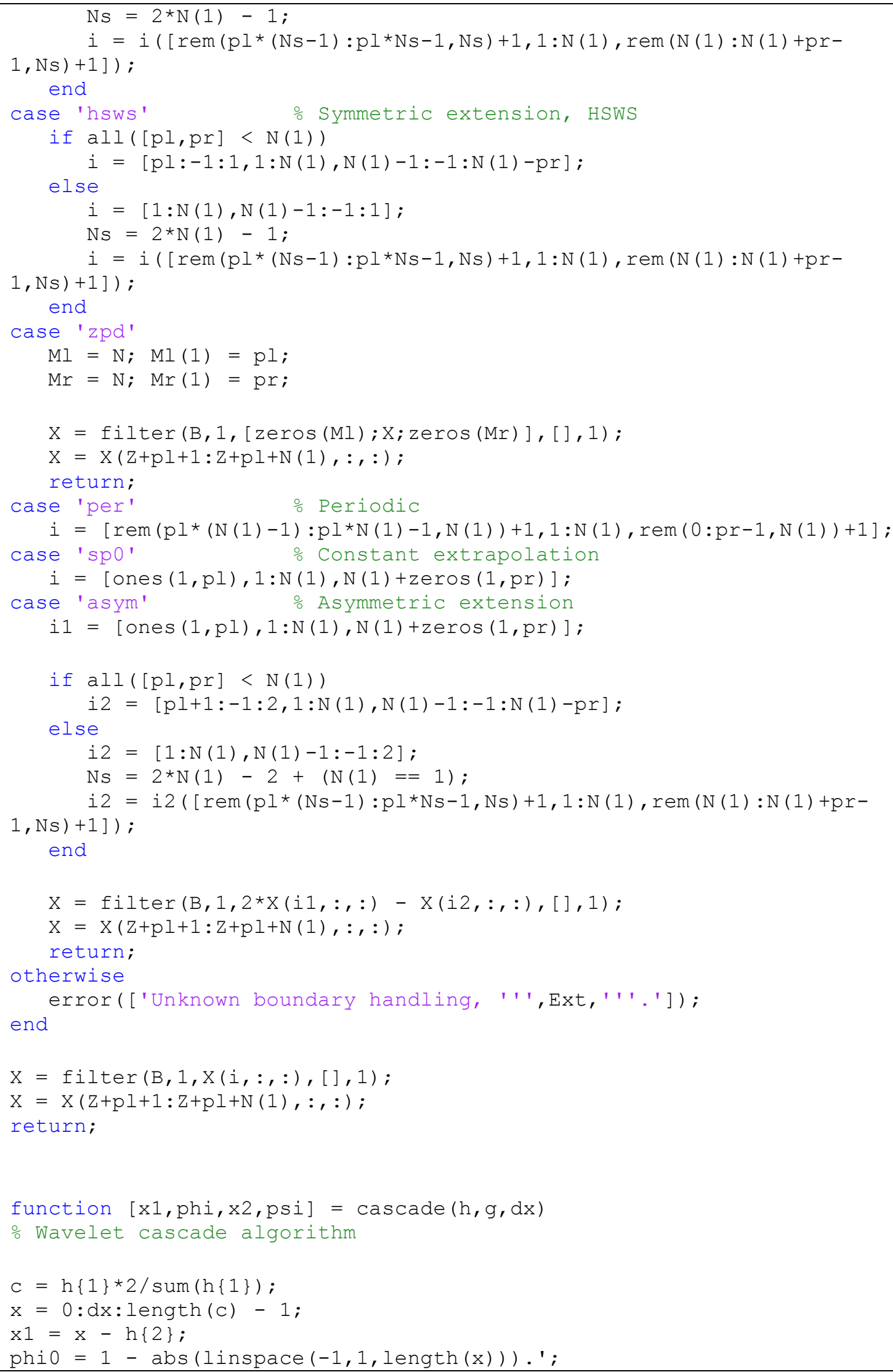




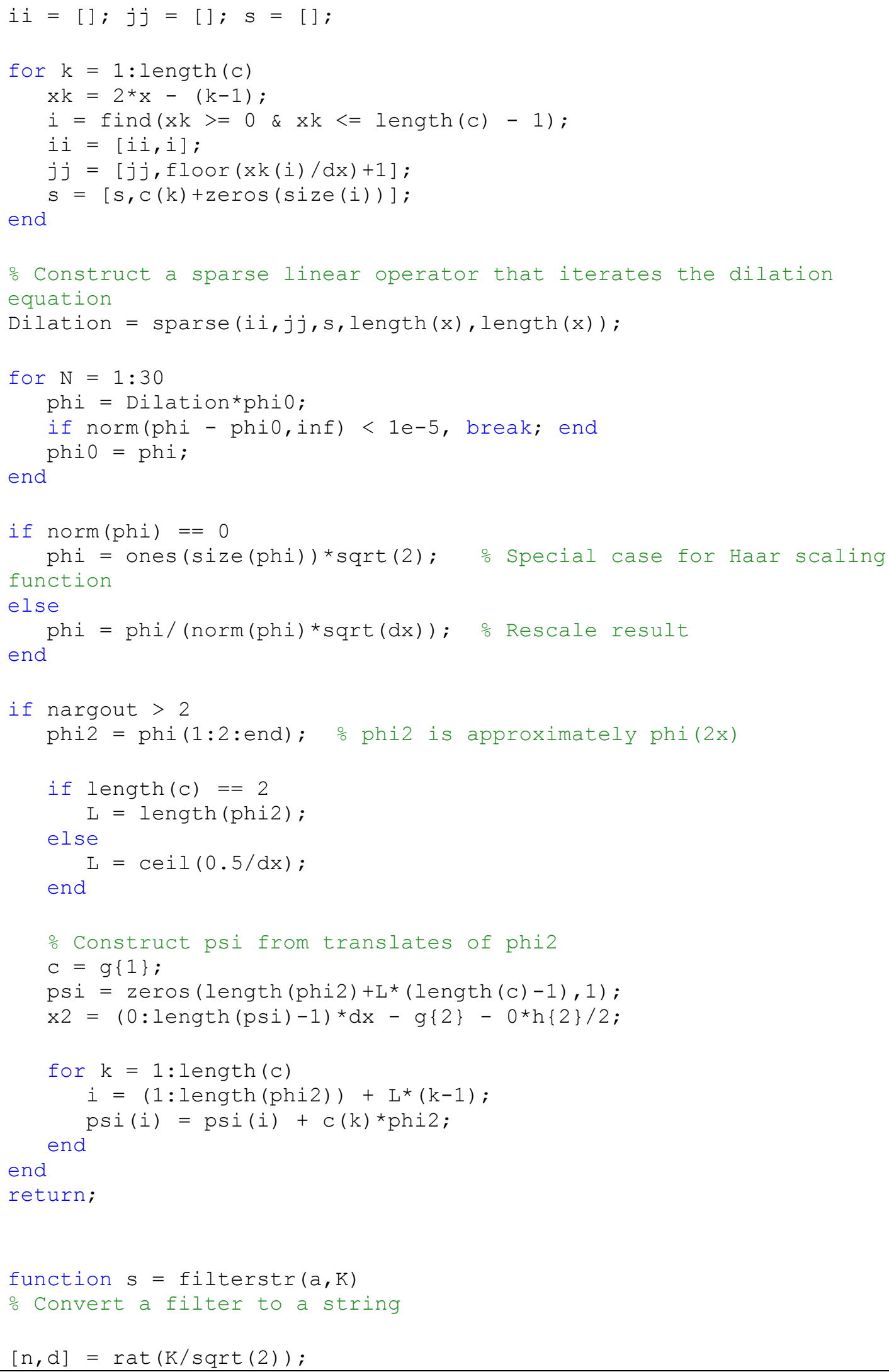




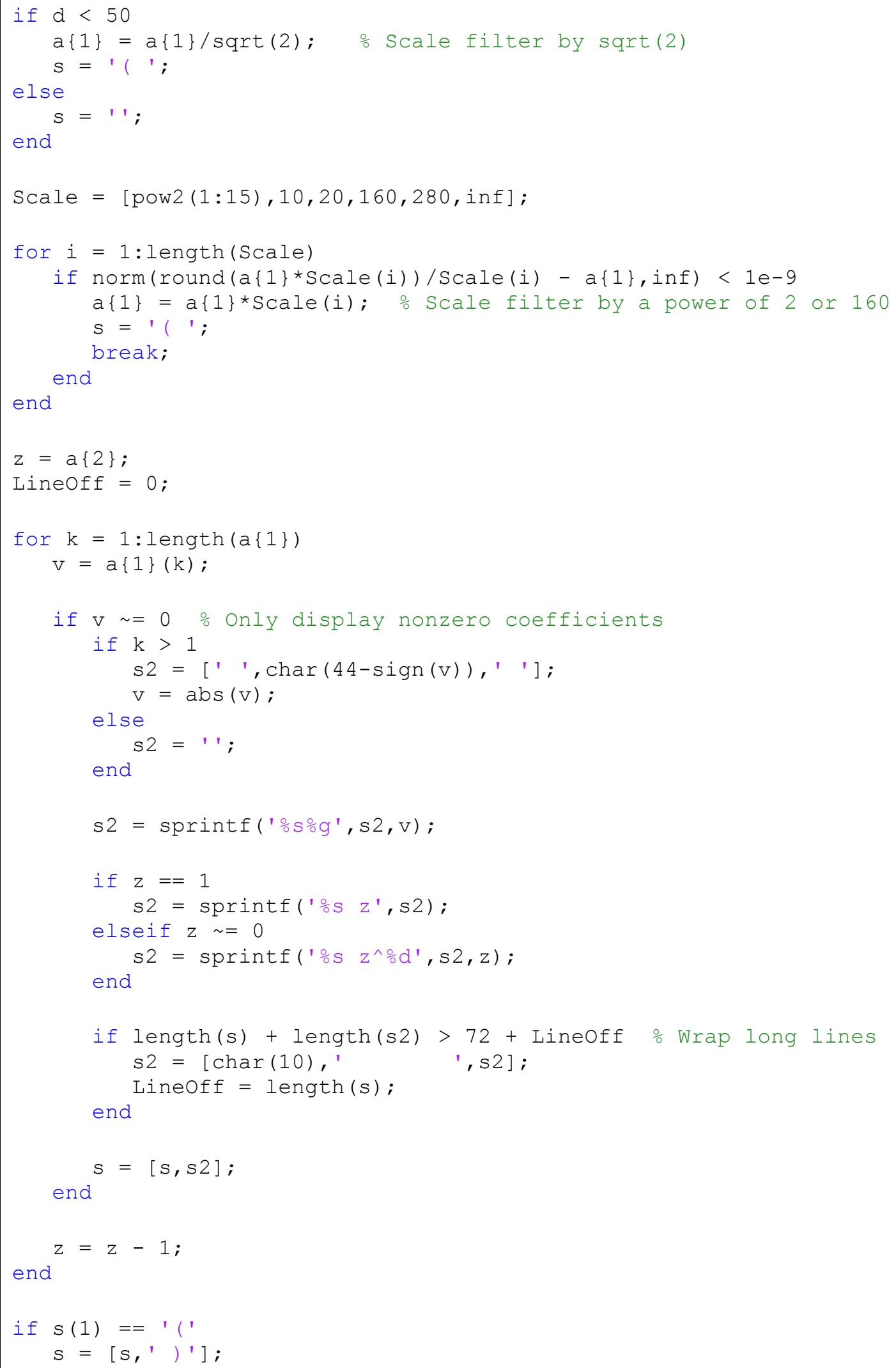




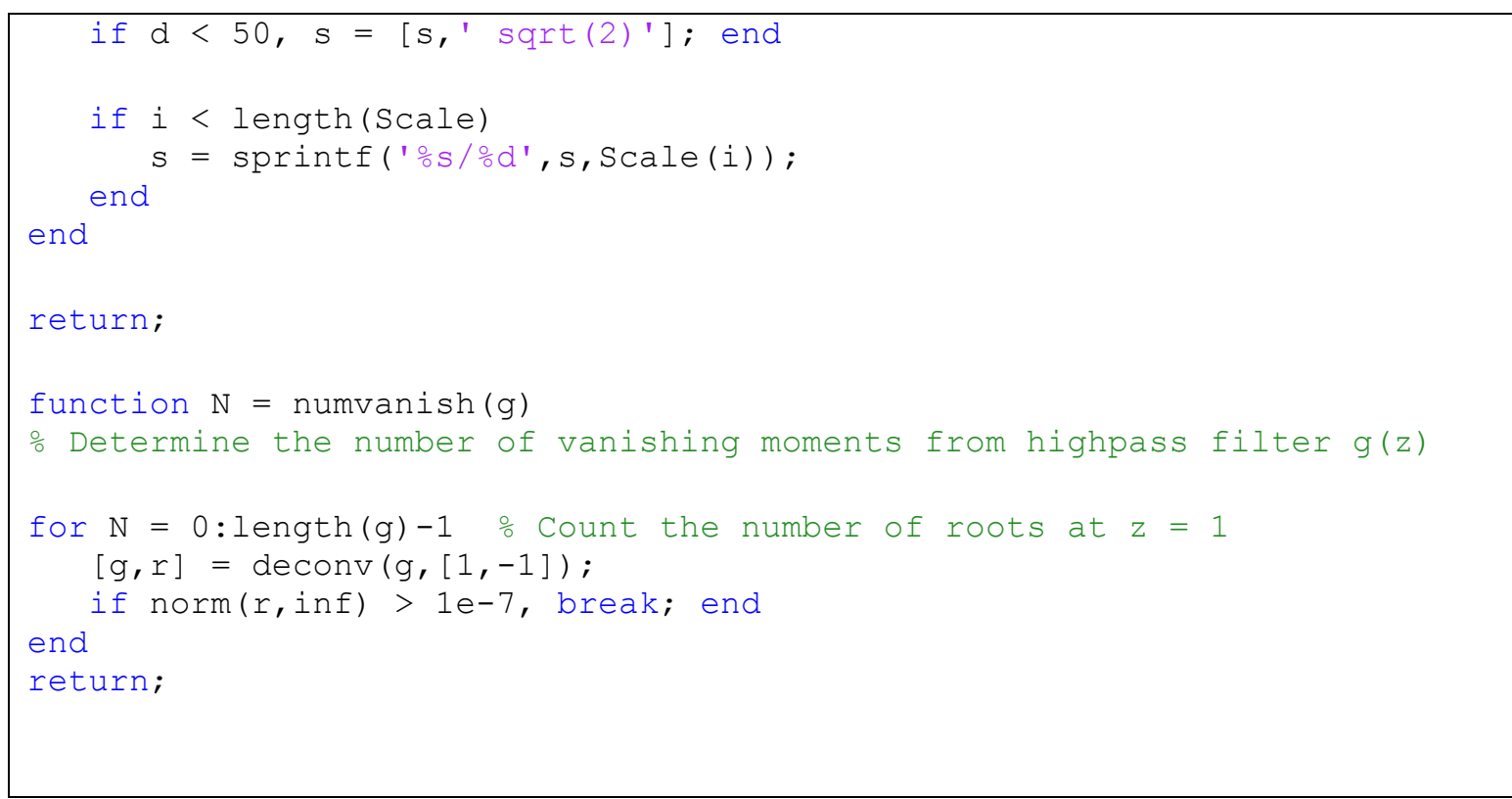

